NASA Contractor Report 3062

\title{
A Higher Order Panel Method for Linearized Supersonic Flow
}

F. Edward Ehlers, Michael A. Epton,

Forrester T. Johnson, Alfred E. Magnus, and Paul E. Rubbert

CONTRACT NAS2-7729

MAY 1979

\section{FOR EARLY DOMESTIC DISSEMINATION}

Because of its significant early commercial potential, this information, which has been developed under a U.S. Government program, is being disseminated within the United States in advance of general publication. This information may be duplicated and used by the recipient with the express limitation that it not be published. Release of this information to other domestic parties by the recipient shall be made subject to these limitations.

Foreign release may be made only with prior NASA approval and appropriate export licenses. This legend shall be marked on any reproduction of this information in whole or in part.

Date for general release.

\section{December 1980}




\section{FEDD DOCUMENT}

Note that this document bears the label "FEDD," an acronym for "FOR EARLY DOMESTIC DISSEMINATION." The FEDD label is affixed to documents that may contain information having high commercial potential.

The FEDD concept was developed as a result of the desire to maintain U.S. leadership in world trade markets and encourage a favorable balance of trade. Since the availability of tax-supported U.S. technology to foreign business interests could represent an unearned benefit, research results that may have high commercial potential are being distributed to U.S. industry in advance of general release.

The recipient of this report must treat the information it contains according to the conditions of the FEDD label on the front cover. 
NASA Contractor Report 3062

\section{A Higher Order Panel Method for Linearized Supersonic Flow}

F. Edward Ehlers, Michael A. Epton, Forrester T. Johnson, Alfred E. Magnus, and Paul E. Rubbert

Boeing Commercial Airplane Company

Seattle, Washington

Prepared for

Ames Research Center

under Contract NAS2-7729

\section{N/SA}

National Aeronautics

and Space Administration

Scientific and Technical

Information Otfice

1979 


\section{CONTENTS}

\section{Page}

1.0 SUMMARY . . . . . . . . . . . . . . . . . . . . . . . . . . . . . . 1

2.0 INTRODUCTION . . . . . . . . . . . . . . . . . . . . . . 2

2.1 Historical Development of Panel Methods . . . . . . . . . . . . . . 2

2.2 Technical Approach and Development of the Present Supersonic

Panel Method . . . . . . . . . . . . . . . . . . . . . . . 4

3.0 THEORY OF LINEARIZED COMPRESSIBLE FLOW . . . . . . . . . . . 6

3.1 Derivation of Basic Equations . . . . . . . . . . . . . . . . . . . 6

3.2 Uniqueness of the Mass Flux Boundary Conditions in Subsonic Flow . . . . 8

3.3 Euler Momentum Theorem for Mass Flux Boundary Conditions

in Linearized Theory .. . . . . . . . . . . . . . . . . . . . . 11

3.4 Integral Equations for Supersonic Linearized Flow . . . . . . . . . . . 14

3.5 Integral Equation for Superinclined Panels . . . . . . . . . . . . . . . 18

3.6 Uniqueness of the Solutions with Potential and Linear Mass Flux

Boundary Conditions on Superinclined Surfaces . . . . . . . . . . . . 20

3.7 Integral Equation for Subinclined Panels . . . . . . . . . . . . . . . 23

3.8 Uniqueness of Solution on Subinclined Surfaces . . . . . . . . . . . . 26

3.9 The Velocity Components Neglecting Vortices at Panel Edges . . . . . . 30

3.10 Boundary Conditions on Wings and Bodies . . . . . . . . . . . . . . 31

4.0 GENERAL DESCRIPTION OF THE SUPERSONIC PANEL METHOD . . . . . 33

4.1 Earlicr Six Parameter Splinc Method . . . . . . . . . . . . . . . . 33

4.2 Improved Nine Parameter Doublet Spline and Improved Paneling

To Obtain Continuous Doublet Strength and Geometry . . . . . . . . . . 35

4.3 Combined Source and Doublet Panels . . . . . . . . . . . . . . . . 36

4.4 Efficient Computations of the Influence Coefficients . . . . . . . . . . 37

5.0 AERODYNAMIC INFLUENCE COEFFICIENTS FOR SUBINCLINED

DOUBLET PANELS . . . . . . . . . . . . . . . . . . . . . . . . . . . . . . 39

6.0 PANEL EDGE SINGULARITIES FOR DOUBLET PANELS . . . . . . . . . . 45

7.0 INFLUENCE COEFFICIENTS FOR SUBINCLINED DOUBLET PANELS NEGLECTING THE EDGE VORTEX . . . . . . . . . . . . . . . . . 49

8.0 AERODYNAMIC INFLUENCE COEFFICIENTS FOR SUBINCLINED SOURCE PANELS . . . . . . . . . . . . . . . . . . . . . . . . . 54

9.0 AERODYNAMIC INFLUENCE COEFFICIENTS FOR SUPERINCLINED

PANELS . . . . . . . . . . . . . . . . . . . . . . . . . . 57

9.1 Velocity Potential for Source and Doublet Panels . . . . . . . . . . . 57

9.2 Velocity Components for the Doublet Distribution Without

Edge Vortices . . . . . . . . . . . . . . . . . . . . . . . 63 


\section{CONTENTS (continued)}

Page

9.3 Velocity Components for Source Distribution . . . . . . . . . . 68

10.0 TRANSFORMATION OF VELOCITY COMPONENTS FROM LOCAL PANEL

TO THE GLOBAL COORDINATE SYSTEM . . . . . . . . . . . . . . . 70

10.1 Transformation From the Reference Coordinate System to the

Compressible System . . . . . . . . . . . . . . . . . . . . . . 70

10.2 Transformation From Compressible Coordinates to Panel Coordinate

System Without the Compressibility Factor B in the Panel System . . . . . 73

10.3 Correction to Panel Element of Area in Supersonic Flow Due to the

Transformation From Global to Panel Coordinates . . . . . . . . . . . 79

10.4 Transformation of Velocity Components to Components in the

Reference System . . . . . . . . . . . . . . . . 81

11.0 FAR FIELD EXPANSIONS FOR THE AERODYNAMIC INFLUENCE

COEFFICIENTS . . . . . . . . . . . . . . . . . . . . . . . 83

11.1 Source Aerodynamic Influence Coefficients . . . . . . . . . . . . . 83

11.2 Doublet Aerodynamic Influence Coefficients . . . . . . . . . . . . . 86

12.0 DISCUSSION OF RESULTS . . . . . . . . . . . . . . . . . . . . . 88

12.1 Source Panel Method Applied to Cones and Bodies of Revolution . . . . . 88

12.2 Source Panel Method Applied to Elliptic Cones . . . . . . . . . . . . 88

12.3 Application of Source and Doublet Panel Methods to Planar Wings

with Linearized Boundary Conditions . . . . . . . . . . . . . . . . 89

12.4 Application of Combined Source and Doublet Panels with Potential Boundary Conditions to Three Dimensional Configurations . . . . . . . 90

12.5 Application of the Continuous Doublet Spline to Three Dimensional Configurations . . . . . . . . . . . . . . . . . . . . . . . . . 91

12.6 Application of the Continuous Doublet Spline With Potential Boundary Conditions to Supersonic Flow Over Wing-Body Configurations . . . . . . . 94

12.7 Application of Superinclined Panels to Close Inlets for the Computation of Supersonic Flow over Nacelles with the Combined Source and Doublet

Panel Method . . . . . . . . . . . . . . . . . . . . . 95

13.0 CONCLUSIONS . . . . . . . . . . . . . . . . . . . . . . . 97

APPENDIX A. DERIVATION OF BASIC FUNCTIONS AND THEIR

DERIVATIVES . . . . . . . . . . . . . . . . . . 98

APPENDIX B. DERIVATION OF THE INFLUENCE COEFFICIENTS FOR SUBINCLINED PANELS OF SMALL RELATIVE CURVATURE . . 116

APPENDIX C. DESCRIPTION OF THE SPLINES FOR SUPERSONIC SOURCE AND DOUBLET PANELS

APPENDIX D. DESCRIPTION OF THE PILOT CODE FOR COMPUTING LINEARIZED SUPERSONIC FLOW OVER AIRCRAFT CONFIGURATIONS 143 


\section{CONTENTS (concluded)}

Page

APPENDIX E. RELATIONSHIP OF SUPERSONIC PANEL IN INFLUENCE

COEFFICIENTS TO SUBSONIC PANEL INFLUENCE

COEFFICIENTS ...................... 148

REFERENCES . . . . . . . . . . . . . . . . . . . . . . . . . 153

FIGURES . . . . . . . . . . . . . . . . . . . . . . . . . 156 


\section{NOTATION AND SYMBOLS}

$\left\{\mathrm{A}_{\mathrm{c}}\right\}$

$\left\{\mathrm{A}_{\mathrm{r}}\right\}$

$A_{T}(i)$

B

$\{\mathrm{B}\}$

c

$\mathrm{C}_{\mathrm{P}}$

$\overline{\mathrm{F}}$

$\mathrm{H}_{\mathrm{k}}(\theta)$

$\mathrm{I}_{\mathrm{n}}$

$\mathrm{m}$

$m_{1}$

$\mathrm{M}$

$\hat{n}$

$\overline{\mathrm{n}}_{\mathrm{c}}$

$\mathrm{n}_{\mathrm{x}_{\mathrm{c}}}, \mathrm{n}_{\mathrm{y}_{\mathrm{c}}}, \mathrm{n}_{\mathrm{z}_{\mathrm{c}}}$

$p$

$P_{n}$

$\mathrm{Q}_{\mathrm{I}}$

$\mathrm{Q}_{\mathrm{m} 0}$
Matrix of transformation from compressible to panel coordinate system

Matrix of transformation from reference to compressible coordinate system

ith row of Matrix $\mathrm{A}_{\mathrm{r}}$

$=\sqrt{M^{2}-1}$

Matrix relating normal to conormal

velocity of sound

$=\left(\mathrm{p}-\mathrm{p}_{0}\right) / 1 / 2 \rho_{0} \mathrm{U}_{0}^{2}$ Pressure coefficient

Force on surface

$=\sqrt{\mathrm{x}^{2}-\mathrm{r}_{\mathrm{k}}(\theta)^{2}}$ Hyperbolic distance from kth panel edge $\mathrm{r}=\mathrm{r}_{\mathrm{k}}(\theta)$

$=\int_{0}^{s_{m}} \frac{t^{n} d t}{\sqrt{t(t+2 r)}}$

$=\left(\mathrm{y}_{2}-\mathrm{y}_{1}\right) /\left(\mathrm{x}_{2}-\mathrm{x}_{1}\right)$

Parameter in oblique transformation

Free stream Mach number

$=\mathrm{n}_{\mathrm{x}}, \mathrm{n}_{\mathrm{y}}, \mathrm{n}_{\mathrm{z}}$ Unit normal vector

$=\left(1-M^{2}\right) n_{x}, n_{y}, n_{z}$ Conormal vector

Components of unit normal in compressible coordinate system

Pressure, Newtons/square meter

$=z \int s^{n} R d s / r^{2}$

Replaces $z Q_{m 0}$ and defined by equation (A19)

$=\mathrm{x}_{\mathrm{m}} \mathrm{Q}_{0}+\mathrm{Q}_{1} / \mathrm{m}$ 
$=\sqrt{\left(y-y_{0}\right)^{2}+z-z_{0}^{2}}$

$=\sqrt{s^{2}+z^{2}}$ in Aerodynamic influence coefficients

Also polar coordinate on superinclined panel

$\overline{\mathrm{R}}$

$\mathrm{R}$

$\mathrm{R}_{1}$

$\mathrm{R}_{\mathrm{B}}$

$\mathrm{R}_{\mathrm{C}}$

$\mathrm{R}_{\mathrm{s}}$

$\mathrm{R}_{\mathrm{i} 1}$

$\mathrm{S}$

$\mathrm{s}_{\mathrm{m}}$

$\mathrm{S}_{\mathrm{n}}$

$\mathrm{s}_{\mathrm{Z}}$

$\overline{\mathrm{U}}$

$\mathrm{u}, \mathrm{v}, \mathrm{w}$

$v_{i}$

$\overline{\mathrm{V}}_{\mathrm{c}}$

$\overline{\mathrm{V}}$

$\overline{\mathrm{W}}$

$\mathrm{w}_{\mathrm{n}}$

$\widetilde{\mathrm{w}}_{\mathrm{n}}$

$\mathrm{w}_{\mathrm{mn}}$

$\hat{\mathrm{x}}_{\mathrm{m}}$
General point position vector in section 3

$=\sqrt{\mathrm{s}_{\mathrm{m}}^{2}-\mathrm{s}^{2}-\mathrm{z}^{2}}$ Hyperbolic distance in aerodynamic influence coefficient functions for subinclincd panels

$=\mathrm{n}-\mathrm{y}, \zeta-\mathrm{z}$ Position coordinate in plane of superinclined panel $=\sqrt{\left(x-x_{0}\right)^{2}-B^{2}\left(y-y_{0}\right)^{2}-B^{2}\left(z-z_{0}\right)^{2}}$ Hyperbolic distance

Farfield hyperbolic distance from control point to panel center

$=\sqrt{x^{2}-(n-y)^{2}-(\zeta-z)^{2}}$ Hyperbolic distance for superinclined panels

$=\int s^{n} R d s$

$=\mathrm{y}-\mathrm{y}_{0} ;$ also indicates infinitesimal area $\mathrm{ds}$.

$=\mathrm{x}_{\mathrm{m}}+\mathrm{s} / \mathrm{m}$

$=\int \mathrm{s}^{\mathrm{n}} \mathrm{I}_{0}\left(\mathrm{r}, \mathrm{s}_{\mathrm{m}}-\mathrm{r}\right) \mathrm{ds}$

$=s x_{m}+z^{2} / m$

Free stream velocity vector, meters/sec.

$\mathrm{x}, \mathrm{y}, \mathrm{z}$ perturbation velocity components, meters/sec.

ith perturbation component, meters/sec.

Complete velocity vector, meters/sec.

Perturbation velocity vector, meters/sec.

Linear perturbation mass flux vector, same dimension as velocity

$=\int \mathrm{s}^{\mathrm{n}} \mathrm{ds} / \mathrm{R}$ for subinclined panels

$=\int \zeta^{\mathrm{n}} \mathrm{d} \zeta / \mathrm{R}_{\mathrm{s}}$ for superinclined panels

$=\mathrm{x}_{\mathrm{m}} \mathrm{w}_{\mathrm{n}}+\mathrm{w}_{\mathrm{n}+\mathrm{l}} / \mathrm{m}$. (Similar notation for other functions)

$=\mathrm{x}-\mathrm{x}_{1}+\left(\mathrm{y}-\mathrm{y}_{1}\right) / \mathrm{m}, \mathrm{x}_{\mathrm{m}}=0$ is panel edge 


$\begin{array}{ll}\mathrm{x}_{\mathrm{m}} & =\mathrm{s}_{\mathrm{m}}-\mathrm{s} / \mathrm{m} \\ \hat{\mathrm{x}}_{\mathrm{m}} & =\mathrm{mx}_{\mathrm{m}} \text { for } \mathrm{m}<1 \\ \tilde{\mathrm{x}}_{\mathrm{i}} & \mathrm{x}_{\mathrm{j}} \mathrm{c}_{\mathrm{ij}} \text { Farfield panel coordinates } \\ \mathrm{x}_{\mathrm{C}}, \mathrm{y}_{\mathrm{c}}, \mathrm{z}_{\mathrm{c}} & \text { Compressible coordinate system } \\ \mathrm{x}_{\mathrm{r}}, \mathrm{y}_{\mathrm{r}}, \mathrm{z}_{\mathrm{r}} & \text { Reference coordinate system of configuration } \\ \mathrm{y}_{\mathrm{e}}, \mathrm{z}_{\mathrm{e}} & \text { Panel edge coordinates for superinclined panels } \\ \mathrm{y}_{\mathrm{m}} & =\mathrm{s}-\mathrm{s}_{\mathrm{m}} / \mathrm{m} \text { Oblique coordinate to } \mathrm{x}_{\mathrm{m}} \\ \hat{y}_{\mathrm{m}} & =\mathrm{my} \mathrm{m}_{\mathrm{m}}=\mathrm{ms}-\mathrm{s}_{\mathrm{m}}, \text { for } \mathrm{m} /<1 \\ \mathrm{z}_{\mathrm{m}} & =\mathrm{x}_{\mathrm{m}}{ }^{2}+\mathrm{z}^{2} / \mathrm{m}^{2}, \hat{z}_{\mathrm{m}}=\mathrm{m}^{2} \mathrm{z}_{\mathrm{m}}=\hat{\mathrm{x}}_{\mathrm{m}}{ }^{2}+\mathrm{z}^{2} \\ \mathrm{z}_{\mathrm{r}} & =\left(\mathrm{y}_{\mathrm{m} 1} \mathrm{R}_{2}-\mathrm{y}_{\mathrm{m} 2}{ }^{\mathrm{r} 1}\right) /\left[\mathrm{y}_{\mathrm{m} 1} \mathrm{y}_{\mathrm{m} 2}+\left(1-\lambda^{2}\right) \mathrm{R}_{1} \mathrm{R}_{2}\right] \\ \mathrm{z}_{\mathrm{R}} & =\mathrm{R} /\left(\mathrm{s}_{\mathrm{m}}-\mathrm{ms}\right)\end{array}$

\section{GREEK SYMBOLS}

$\alpha_{\mathrm{c}}$

Angle of attack of free stream velocity vector with respect to reference coordinate system

$\beta_{\mathrm{c}}$

Angle of yaw of free stream with respect to reference coordinate system

$\beta_{\mathrm{m}}$

$=\sqrt{1-m^{2}}$

$\beta$

$=\sqrt{1-M^{2}}$

$\theta$

$\theta_{1}, \theta_{2}$

Polar coordinate angle

$\sigma$

$\lambda$

$\lambda_{\mathbf{i}}$

Angles of rotation in transformation to panel coordinate system

Source strength

$=1 / \mathrm{m}$

Set of singularity values associated with the panel system

$\mu$

Doublet strengtlı

Density, kilograms $/$ meter $^{3}$ 
$=1 / \mathbf{R}_{\mathrm{S}}$

$\phi$

Perturbation velocity potential in meters

$\phi_{\mathbf{i}}$

Equivalen incompressible velocity potential in meters

$\xi, \eta$

Variables of integration over planar subinclined panel

$\eta, \xi$

Variables of integration over planar subinclined panel

$\nabla$

$=\frac{\partial}{\partial x}, \frac{\partial}{\partial y}, \frac{\partial}{\partial z} \quad$ Vector differential operator

\section{NOTATION}

Subscripts 1 and 2 used to designate quantities evaluated at points 1 and 2 of panel edge
$\partial / \partial n_{c}$
Conormal derivative
$\pm$
Denotes upper and lower sides of surface when used as superscripts
"
Denotes finite part of integral
$-1$
As superscript on matrix denotes inverse
$\mathrm{T}$
As superscript on matrix denotes transpose
$\nabla$
$=(\partial / \partial x, \partial / \partial y, \partial / \partial z)$
$\widetilde{\nabla}$
$=(-\partial / \partial \xi, \partial / \partial n, \partial / \partial \xi)$
$\nabla^{\prime}$
$=(\partial / \partial \xi, \partial / \partial \eta, \partial / \partial \zeta)$ or sometimes $(\partial / \partial \eta, \partial / \partial \zeta)$
Proportional to 


\subsection{SUMMARY}

The development of an advanced supersonic panel method is described in detail. The basic integral equations of linearized supersonic theory are derived with a discussion on boundary conditions providing uniqueness of the solution. Because of the success of the method of Johnson and Rubbert for subsonic flow, their geometry and spline system was first utilized by essentially replacing the subsonic aerodynamic influence coefficients by the equivalent supersonic relations.

The source method first was tested on a variety of regular three dimensional bodies, including circular and elliptic cones and pointed bodies of revolution. The calculated results for circular cones and bodies of revolution were in agreement with exact theory; and for elliptic cones, good agreement with second order linear theory was found. The 6 parameter doublet panel method was tested on cambered wings with zero thickness and linearized boundary conditions and gave excellent agreement with exact linearized solutions.

Using source paneling on both surfaces of thin wings and on inlet nacelles lead to the discovery that internal wave reflections induced severe perturbations on the exterior pressure distributions. These perturbations were eliminated by using a combined source and doublet panel system with interior boundary conditions specified to eliminate the internal perturbation flow.

An analysis of the aerodynamic influence coefficients indicated that discontinuities of the doublet strength, or of geometry at panel edges, introduce infinite square root singularities on the Mach cones emanating from panel corners. For certain panel configurations these singularities produced large oscillations in the pressure, even though the doublet strength was very nearly continuous across panel edges. On the first attempt to eliminate this problem, the line integrals along panel edges which involve the doublet strength and which contain the strongest singularities were discarded; but the method proved inaccurate, indicating a strong sensitivity to continuity of doublet strength.

It became apparent that to insure sufficient accuracy and stability the supersonic panel method must maintain continuity of both the doublet strength and geometry. A panel system with all contiguous edges was obtained by dividing the basic four point non-planar panel into eight triangular subpanels. A quadratic doublet distribution is applied over each triangular subpanel in such a way that the doublet strength is continuous at panel edges, leading to a new nine parameter spline for the complete panel. This new spline, with combined source and doublet panels to eliminate internal perturbations, was used to compute the flow over three wing-body models and yielded pressure coefficients in good agreement with experiment. The present method appears to be insensitive to how the configuration is paneled, provided no gaps occur at panel edges and no subpanels representing solid surfaces are inclined at angles with respect to the free stream greater than the Mach angle.

To close inlets on nacelles for the purpose of eliminating internal flows, superinclined panels were developed and tested on a simple nacelle. The superinclined network was capable of absorbing the internal perturbations from the lip of the nacelle. With the addition of the superinclined networks, the panel method is now capable of computing the flow over wing and body combinations which include engine inlets as well.

For those readers interested only in a simple overall view of the method and the results, section 2 the introduction, section 3 on theory, section 4 on the description of the panel method and sections 12 and 13 on results and conclusions may be understood without the details provided by the remaining sections. 


\subsection{INTRODUCTION}

\subsection{HISTORICAL DEVELOPMENT OF PANEL METHODS}

Methods based on linearized theory with singularity distributions over panels representing aircraft surfaces have been found especially useful tools for analyzing the aerodynamic forces on aircraft. These methods can treat configurations of general shape which are not tractible by direct mathematical analysis. Boundary conditions are applied at discrete points associated with each panel of the surface. The required integrais are usually evaluated in closed form, and a set of linear equations results to be solved for the required parameters. These methods have been especially successful for subsonic or incompressible flow and since a good summary is given in Ashley and Rodden [1] and Rubbert and Saaris [2], they will not be discussed here. The most recent method is that of Jolnnson and Rubbert [3]. Much of the technique for supersonic flow presented here is derived from their method.

In recent years, there are three panel methods based on linearized supersonic flow which are noteworthy. Woodward [4] used constant pressure and constant source panels and applied tangential flow boundary conditions on wings and bodies. His method was later improved by utilizing constant line sources and vortices which vary linearly in the streamwise direction on each panel (reference [5]).

To obtain differentiability of the computed pressure, Mercer, Weber, and Lesferd [6] used singularity splines providing continuity of the vorticity. Difficulties arose at discontinuities in the plan form for the vorticity method which were alleviated by rounding such corners. Piecewise linear pressure distributions were obtained for planar wings with linear boundary conditions instead of the stepwise distribution from the constant singularity panel methods.

Morino, Chen, and Suciu [7] describe a method using Green's formula for the potential. The boundary conditions of tangential flow are not applied directly to the potential in the useful way but are inserted into the source term in Green's theorem. Requiring continuity of tangential flow near the surface insures that the boundary conditions are satisfied. The configuration is divided into quadrilaterals defined by an array of grid points on the surface. The surface is approximated for eacl panel by fitting a hyperboloidal surface through the four points which maintains geometric continuity with adjacent panels. The quantities, such as values of doublet and source strengths, were assumed constant on each panel leading to an influence coefficient method with a set of linear equations to be solved for the values of the unknown doublet strengths.

In the appendix to reference [6], Mercer, Weber, and Lesferd suggest a doublet Mach line panel method using a fourth degree polynomial in characteristic coordinates for the doublet distribution on each panel to analyze planar wings. This type of paneling has a number of positive features:

1. Discontinuities in pressure occur across Mach lines from planform corners and can be easily taken into account with Special Mach line paneling. 
2. The aerodynamic influence coefficients in the characteristic coordinates are very simple, closed form expressions.

3. Because of the domain of influence of the characteristic strips, the matrix of coefficients for the parameters can be made triangular by proper ordering, and hence the solution is exceedingly fast.

4. A continuous pressure distribution is obtained.

This method, along with a similar source panel technique, was derived and tested as part of the present contract. The basic theory and results are presented in a scparate contractors report (reference [8]). The source panel method was stable and accurate for both analysis and design boundary conditions. The doublet method was also stable for the region downstream of the Mach line from the corner formed by the supersonic leading edge but was unstable for the region downstream of the Mach line from the corner formed by the supersonic and subsonic portions of the leading edge. The cause of this instability was not pursued, principally because the method was confined to planar wings with linearized boundary conditions and hence has limited application, but also because the subsonic method of Johnson and Rubbert [3] appeared to be a more promising approach.

The most advanced panel method for subsonic flow is that developed by Johnson and Rubbert [3]. The technique uses a quadratic approximation for curved panels with a quadratic distribution of doublet strength and a linear distribution of source strength applied to each panel. The vanishing of the normal component of the mass flux is applied as boundary conditions to solid surfaces instead of the vanishing of the normal component of velocity as in most of the earlier methods. This is discussed in section 3. The aerodynamic influence coefficients are correct to the first order in relative panel curvature. The method has the following properties:

1. It is insensitive to how the configuration is paneled; and, hence, allows the use of automatic paneling programs. It does not require special experience in applying the program to practical engineering problems.

2. It is economical in computation cost. For a given accuracy, the method requires fewer panels than the constant singularity panel techniques.

3. It offers a wide variety in the choice of modeling techniques, including both design and analysis boundary conditions.

The numerical method is stable, accurate, flexible, and efficient, essential properties for any numerical method to be useful and practical for engineering design and analysis. It was felt that the approach was ideal for supersonic flow as well. The techniques for treating the geometry and the mode of paneling in the subsonic method were utilized with very little change in the initial supersonic method, along with the quadratic doublet and linear source distribution on each panel. In subsonic flow, disturbances due to discontinuities of the doublet strength at panel edges or gaps in panel geometry decrease in intensity with distance from their origin. In supersonic flow, similar discontinuities produce disturbances which do not decay with distance but introduce infinite singularities along Mach cones emanating from panel 
corners. Hence, continuity requirements for supersonic doublet panels are considerably more strict than for subsonic. To overcome this difficulty, a paneling system with contiguous edges and a doublet spline with continuous doublet strength were developed.

\subsection{TECHNICAL APPROACH AND DEVELOPMENT OF THE PRESENT SUPERSONIC PANEL METHOD}

With the considerable success of the subsonic panel method of Johnson and Rubbert [3], it was natural that the same method of paneling and the same splines be utilized directly with the supersonic aerodynamic influence coefficients replacing the subsonic ones. The basic theory for linearized supersonic flow is presented in section 3 . The perturbation potential is derived as an integral over the surface of sources and doublets in section 3.7 in the form

$$
\phi(\overline{\mathrm{R}})=-\frac{1}{2 \pi} \int_{S_{W}} \frac{\sigma\left(\overline{\mathrm{R}}_{0}\right) \mathrm{ds}}{\mathrm{R}_{\mathrm{B}}}+\frac{\mathrm{B}^{2}}{2 \pi} \quad \int_{\mathrm{S}_{\mathrm{W}}} \frac{\mu\left(\overline{\mathrm{R}}_{0}\right)\left(\overline{\mathrm{R}}_{0}-\overline{\mathrm{R}}\right) \cdot \hat{\mathrm{n}} \mathrm{ds}}{\mathrm{R}_{\mathrm{B}}{ }^{3}}
$$

where the asterisk indicates finite part (see section 3.4), $\sigma$ is the source strength, $\mu$ the doublet strength,

$$
R_{B}=\sqrt{\left(x-x_{0}\right)^{2}-B^{2}\left(y-y_{0}\right)^{2}-B^{2}\left(z-z_{0}\right)^{2}}
$$

is the hyperbolic distance, $\bar{R}=(x, y, z), \bar{R}_{0}$, the integration point, and $B=\sqrt{M^{2}-1}$, and $\hat{n}$ $=\left(n_{x}, n_{y}, n_{z}\right)$ is the unit normal vector to the surface.

The boundary conditions that the normal component of the linearized mass flux vector vanish on solid surfaces is given by the combination of equations (3.16), (3.12), and (3.8) and takes the form

$$
\left(1-\mathrm{B}^{2} \phi_{\mathrm{X}}\right) \mathrm{n}_{\mathrm{x}}+\phi_{\mathrm{y}} \mathrm{n}_{\mathrm{y}}+\phi_{\mathrm{z}} \mathrm{n}_{\mathrm{z}}=0
$$

for the freestream velocity in the $\mathrm{x}$ direction. This is the basic boundary condition applied to surfaces over which the supersonic flow is to be determined.

The configuration to be analyzed is divided into quadrilateral panels by a set of grid points on the surface. As in reference [3], an average plane is defined by the four midpoints of the lines joining the grid points. A linear source and a quadratic doublet distribution are prescribed over the projection of the panel onto the average plane and the integrals were evaluated in closed form. Associated with each panel, are a value of the source and a value of the doublet strength together with two appropriate boundary conditions. This leads to a set of linear equations to be solved in the form 


$$
\sum_{i=1}^{n} a_{i j} \lambda_{i}=b_{j}, j=1,2, \ldots n
$$

where $a_{i j}$ is the matrix of aerodynamic influence coefficients, $\lambda_{i}$ are the singularity strengths. Once the $\lambda_{i}$ are found the velocity and pressure may bc computed anwhere in the flow field.

Johnson and Rubbert [3] also included a correction to the aerodynamic influence coefficients due to panel curvature, by expanding the potential for a curved panel in terms of the small quantities defining the panel shape and retaining only the first order terms. The same approach failed for supersonic flow because of the singularity on the Mach cone which was of higher order for the curvature terms and, hence, the pressures were non-integrable. With flat panels, the method produced excellent results for a wide range of configurations, and these are reported in section 12 .

Hess [9] has shown that a discontinuity in doublet strength induces the same velocity field as a line vortex. (The relationship between doublet sheets, vortex sheets and line vorlices is explained in Appendix A of reference [9]). Even when the doublet is continuous from one panel edge to the adjoining panel edge, gaps between panels will produce infinite singularities on the Mach cones from the panel corners because the gap prevents the cancelling of the two line vortices. These singularities in supersonic flow do not decay with distance from the edge as in subsonic flow but propagate along Mach cones nearly unattenuated (see section A8.1 in Appendix A). For some paneling configurations, these disturbances impinge upon control points and cause large oscillations in pressure. It was felt that since the discontinuities in doublet strength were small, the problem could be eliminated by discarding the line integrals of the doublet strength along panel edges which produce the line vortices. (For example, the second integral of the equation following equation (7.1)). When this was done the method proved to be inaccurate even for small discontinuities of doublet strength or geometry, although this approach was successful for subsonic flow.

It became apparent that, for supersonic flow, the gaps at panel edges must be eliminated by a panel system that maintains continuity of geometry and by a spline system which ensures continuity of doublet strength. This was achieved by dividing the basic non-planar panel formed by the four points on the curved surface into 8 triangular panels in such a way that no gaps occur in the geometry (see figure 1). A quadratic distribution of doublet strength is prescribed over each triangular subpanel in such a way that doublet strength is continuous over the entire surface. This leads to a 9 parameter spline instead of the former 6 parameter spline; and with the combined source and doublet paneling yields satisfactory solutions for the supersonic flow over complicated wing body configurations.

Section 3 presents a derivation of the basic integral equations of linearized theory with appropriate boundary conditions, while section 4 presents a more detailed description of the panel method. Derivation of specific formulas for the method are given in Section 5 through 11. For those interested only in an overall view of the panel method, section 4 and also sections 12 and 13 giving results and conclusion may be read without the necessity of having read the complete theory. 


\subsection{THEORY OF LINEARIZED COMPRESSIBLE FLOW}

\subsection{DERIVATION OF BASIC EQUATIONS}

To derive the basic equation we follow the analysis of Ward [10] and consider Euler's equations for steady, inviscid flow in the form

$$
\begin{gathered}
\nabla \times \overline{\mathrm{V}}_{\mathrm{c}}=0 \\
\nabla \cdot \rho \overline{\mathrm{V}}_{\mathrm{c}}=0 \\
\rho \overline{\mathrm{V}}_{\mathrm{c}} \cdot \nabla \overline{\mathrm{V}}_{\mathrm{c}}+\nabla \mathrm{p}=0
\end{gathered}
$$

where $\bar{V}_{c}=\bar{U}+\bar{V}, \bar{U}$ is the free stream velocity vector, and $\bar{V}$ the perturbation velocity vector. The quantities $p$ and $\rho$ are the pressure and density, respectively. With the subscript 0 denoting free stream values of the quantities, linearizing of equations (3.1) to (3.3) yields

$$
\begin{gathered}
\nabla \times \overline{\mathrm{V}}=0 \\
\rho_{0} \nabla \cdot \overline{\mathrm{V}}+\overline{\mathrm{U}} \cdot \nabla\left(\rho-\rho_{0}\right)=0 \\
\rho_{0} \overline{\mathrm{U}} \cdot \nabla \overline{\mathrm{V}}+\nabla\left(\mathrm{p}-\mathrm{p}_{0}\right)=0
\end{gathered}
$$

Since for isentropic flow $\mathrm{dp}=\mathrm{c}^{2} \mathrm{~d} \rho \simeq \mathrm{c}_{0}{ }^{2} \mathrm{~d} \rho$, where $\mathrm{c}$ is the velocity of sound, eliminating $\rho$ and $p$ between equations (3.5) and (3.6) gives a single differential equation for the velocity, namely,

$$
\nabla \cdot\left[\overline{\mathrm{V}}-\overline{\mathrm{U}}(\overline{\mathrm{U}} \cdot \overline{\mathrm{V}}) / \mathrm{c}_{0}{ }^{2}\right]=0
$$

or

$$
\nabla \cdot \overline{\mathrm{W}}=0
$$


where $\overline{\mathrm{W}}=\overline{\mathrm{V}}-\mathrm{U}(\overline{\mathrm{U}} \cdot \overline{\mathrm{V}}) / \mathrm{c}_{0}{ }^{2}$ and we have made use of the fact that $\overline{\mathrm{U}}$ is a constant vector. When we assume that $\bar{U}$ is a vector in the $x$ direction, $\bar{W}$ becomes

$$
\mathrm{W}=\left[\left(1-\mathrm{M}^{2}\right) \mathrm{u}, \mathrm{v}, \mathrm{w}\right]
$$

where $\mathrm{M}^{2}=\overline{\mathrm{U}} \cdot \overline{\mathrm{U}} / \mathrm{c}_{0}{ }^{2}$ is the square of the frce stream Mach number. From equation (3.7) we see that the quantity $\bar{W}$ is conserved in the flow. In the following we shall show that $\bar{W}$ is proportional to the perturbation mass flux vector.

To find the local linearized pressure we consider Bernouilli's equation in the form

$$
\int_{\mathrm{p}_{0}}^{\mathrm{p}} \mathrm{dp} / \rho+\overline{\mathrm{V}}_{\mathrm{c}} \cdot \overline{\mathrm{V}}_{\mathrm{c}} / 2=\overline{\mathrm{U}} \cdot \overline{\mathrm{U}} / 2
$$

Expanding $1 / \rho$ in powers of $\mathrm{p}-\mathrm{p}_{0}$

$$
\begin{aligned}
1 / \rho & =1 / \rho_{0}-(\mathrm{d} \rho / \mathrm{dp})_{0}\left(\mathrm{p}-\mathrm{p}_{0}\right) / \rho_{0}{ }^{2}+\ldots \\
& =1 / \rho_{0}-\left(\frac{\mathrm{p}-\mathrm{p}_{0}}{\rho_{0}}\right) \frac{1}{c_{0}^{2} \rho_{0}}+\ldots
\end{aligned}
$$

and substituting into equation (3.9) yields

$$
\frac{\left(p-p_{0}\right)}{\rho_{0}}-\frac{1}{2 c_{0}^{2}}\left(\frac{p-p_{0}}{\rho_{0}}\right)^{2}+\ldots=-\bar{U} \cdot \bar{V}-\bar{V} \cdot \bar{V} / 2
$$

since $\bar{V}_{c}=\bar{U}+\bar{V}$. This can be solved for $\left(p-p_{0}\right) / \rho_{0}$ by inverting the series. Thus, retaining no terms higher than quadratic gives

$$
\mathrm{p}-\mathrm{p}_{0} \quad / \rho_{0}=-\overline{\mathrm{U}} \cdot \overline{\mathrm{V}}-\overline{\mathrm{V}} \cdot \overline{\mathrm{W}} / 2
$$

The quantity $\rho_{\mathrm{o}} \overline{\mathrm{W}}$ is now shown to be the linearized perturbation mass flux vector. Consider the difference between the local mass flux and that for the free stream. This is

$$
\rho \overline{\mathrm{V}}_{\mathrm{C}}-\rho_{0} \overline{\mathrm{U}}=\left(\rho-\rho_{0}\right) \overline{\mathrm{U}}+\rho_{0} \overline{\mathrm{V}}
$$

with

$$
\rho-\rho_{0}=(\mathrm{d} \rho / \mathrm{dp})_{0}\left(\mathrm{p}-\mathrm{p}_{0}\right)+\ldots \simeq\left(\mathrm{p}-\mathrm{p}_{0}\right) / \mathrm{c}_{0}{ }^{2}+\ldots
$$


we have from equation (3.10)

$$
\rho \overline{\mathrm{V}}_{\mathrm{c}}-\rho_{0} \overline{\mathrm{U}}=\rho_{0}\left[\overline{\mathrm{V}}-\overline{\mathrm{U}}(\overline{\mathrm{U}} \cdot \overline{\mathrm{V}}) / \mathrm{c}_{0}{ }^{2}+\ldots\right]=\rho_{0} \overline{\mathrm{W}}+\ldots
$$

Hence equation (3.7) is shown to be the conservation equation for the perturbation mass flux vector.

The conditions of irrotationality, equation (3.1), is satisfied by introducing a velocity potential. Let

$$
\overline{\mathrm{V}}=\mathrm{U}_{0} \nabla \phi
$$

then the conservation equation (3.7) becomes, with $\bar{U}$ in the $x$ direction,

$$
\left(1-\mathrm{M}^{2}\right) \phi_{\mathrm{Xx}}+\phi_{\mathrm{yy}}+\phi_{\mathrm{zz}}=0
$$

and is easily recognized as the classic Prandtl-Glauert equation for linearized compressible flow. From equation (3.10) the pressure coefficient becomes

$$
\mathrm{C}_{\mathrm{p}}=\frac{\mathrm{p}-\mathrm{p}_{0}}{\frac{1}{2} \rho_{0} \mathrm{U}_{0}^{2}}=-2 \phi_{\mathrm{X}}-\left(\beta^{2} \phi_{\mathrm{X}}^{2}+\phi_{\mathrm{y}}^{2}+\phi_{\mathrm{z}}^{2}\right)
$$

where

$$
\beta^{2}=1-M^{2}
$$

When $\beta^{2} \phi_{\mathrm{X}}{ }^{2}$ is dropped this is the slender body approximation to the pressure. For flat wings, the quadratic terms are often neglected. However, we often compute pressure by the slender body approximation or by the complete isentropic relation.

\subsection{UNIQUENESS OF THE MASS FLUX BOUNDARY CONDITIONS IN SUBSONIC FLOW}

To demonstrate uniqueness of the solution of equation (3.7) or (3.13) under certain boundary conditions for subsonic flow, we apply the divergence theorem to $\phi \overline{\mathrm{W}}$ for a volume $\mathrm{V}$ enclosed by a surface S. We obtain, using $\nabla \cdot \bar{W}=0$,

$$
\int_{S} \phi \bar{W} \cdot \hat{n} d s=\int_{V}^{\prime} \bar{W} \cdot \nabla \phi d v=\int_{V}\left(\beta^{2} u^{2}+v^{2}+w^{2}\right) d v
$$


where $\hat{n}=\left(n_{x}, n_{y}, n_{z}\right)$ is the outward normal to the surface $S$. Let $S$ be divided in to two parts with $\phi$ prescribed on $S_{1}$ and $\bar{W} \cdot \hat{n}$ prescribed on $S_{2}$. Since the differential equation is linear, the difference of two solutions is also a solution and equation (3.15) holds. Let $\phi$ and $\bar{W}$ denote the difference of two solutions which satisfy the same prescribed boundary conditions. Then the surface integral on the left hand side of the equation (3.15) vanishes. The volume integral on the right side is identically equal to zero. Since the terms of the integrand are always positive, the integral can be zero only if the terms of the integrand are zero everywhere, and the two solutions are identical. Hence, the solution of $\nabla \cdot \bar{w}=0$ will be unique with $\phi$ or $\overline{\mathrm{W}} \cdot \hat{n}$ prescribed as boundary conditions on the surface $S$. This can be shown for infinite regions when $\phi$ goes to zero like $1 / \mathrm{r}$ as $\mathrm{r}$ goes to infinity. In the light of the uniqueness theorem it is appropriate in our panel methods for analyzing the flow over bodies to use linearized mass flux boundary conditions. On solid boundaries this takes the form

$$
(\overline{\mathrm{U}}+\overline{\mathrm{W}}) \cdot \hat{\mathrm{n}}=0
$$

or for the velocity potential in equation (3.12) and a free stream velocity in the $\mathrm{x}$ direction

$$
\left(1+\beta^{2} \phi_{\mathrm{X}}\right) \mathrm{n}_{\mathrm{x}}+\phi_{\mathrm{y}} \mathrm{n}_{\mathrm{y}}+\phi_{\mathrm{z}} \mathrm{n}_{\mathrm{z}}=0
$$

This equation can be written in a different form by introducing the conormal vector

$$
\overline{\mathrm{n}}_{\mathrm{c}}=\beta^{2} \mathrm{n}_{\mathrm{X}}, \mathrm{n}_{\mathrm{y}}, \mathrm{n}_{\mathrm{z}}
$$

We obtain for equation (3.17);

$$
n_{x}+\partial \psi / \partial n_{c}=0
$$

The conormal derivative of the potential then can be interpreted as the normal component of the perturbation mass flux to the surface.

The differential equation (3.13), the pressure relation in equation (3.14) and boundary conditions in (3.17) comprise the fundamental boundary valuc problem we are solving for analyzing aircraft configurations by the methods described here. When the flow is subsonic, the equations can be converted to the incompressible flow by introducing the incompressible velocity potential

$$
\phi=\phi_{\mathrm{i}} / \beta
$$


and scaling the $\mathrm{x}$ variable by

$$
\mathrm{x} \rightarrow \mathrm{x} / \beta
$$

Since $\mathrm{n}_{\mathrm{x}} \rightarrow \mathrm{n}_{\mathrm{x}} / \beta$, we obtain

$$
\begin{gathered}
\overline{\mathrm{W}}=\left(\phi_{\mathrm{ix}}, \phi_{\mathrm{iy}} / \beta, \phi_{\mathrm{iz}} / \beta\right) \\
\phi_{\mathrm{ixx}}+\phi_{\mathrm{iyy}}+\phi_{\mathrm{izz}}=0 \\
\mathrm{C}_{\mathrm{p}}=\mathrm{C}_{\mathrm{pi}} / \beta^{2} \\
\mathrm{C}_{\mathrm{pi}}=-2 \phi_{\mathrm{ix}}-\left(\phi_{\mathrm{ix}}^{2}+\phi_{\mathrm{iy}}{ }^{2}+\phi_{\mathrm{iz}}^{2}\right) \\
\left(1+\phi_{\mathrm{ix}}\right) \mathrm{n}_{\mathrm{x}}+\phi_{\mathrm{iy}} \mathrm{n}_{\mathrm{y}}+\phi_{\mathrm{iz}} \mathrm{n}_{\mathrm{z}}=0
\end{gathered}
$$

Note that the compressible pressure coefficient including the quadratic terms is related to the incompressible value by the factor $1 / \beta^{2}$. This was first pointed out by Goethert [11]. Equations (3.21) and (3.23) form the classical Neumann boundary value problem for Laplace's equation. Solutions to this problem are unique. This is another proof of uniqueness for the mass flux boundary conditions in linearized subsonic flow. Equations (3.21), (3.22), and (3.23) are the basic equations for the subsonic panel method described in Johnson and Rubbert [3] and which is utilized to a great extent in developing the supersonic method to be presented in the following.

Equation (3.17) and the definition of the conormal hold for supersonic flow as well. Since $M>1$, we obtain

$$
\left(1-\mathrm{B}^{2} \phi_{\mathrm{x}}\right) \mathrm{n}_{\mathrm{x}}+\phi_{\mathrm{y}} \mathrm{n}_{\mathrm{y}}+\phi_{\mathrm{z}} \mathrm{n}_{\mathrm{z}}=0
$$

where $B=\sqrt{M^{2}-1}$. The conormal then takes the form

$$
\overline{\mathrm{n}}_{\mathrm{c}}=-\mathrm{B}^{2} \mathrm{n}_{\mathrm{x}}, \mathrm{n}_{\mathrm{y}}, \mathrm{n}_{\mathrm{z}}
$$




\subsection{EULER MOMENTUM THEOREM FOR MASS FLUX BOUNDARY CONDITIONS IN LINEARIZED THEORY}

We shall show that the mass flux boundary conditions are also compatible with Euler's momentum theorem when $p$, the pressure, is given by equation (3.10). For a closed surface $S$ with no internal sources and for which the velocity vector $\bar{V}_{c}$ is not singular, the theorem takes the general form:

$$
\int_{S}\left[\mathrm{p} \hat{n}+\rho\left(\bar{V}_{\mathrm{c}} \cdot \hat{\mathrm{n}}\right) \overline{\mathrm{V}}_{\mathrm{c}}\right] \mathrm{ds}=0
$$

For linearized flow the mass flux vector $\rho \overline{\mathrm{V}}_{\mathrm{c}}$ becomes

$$
\rho \overline{\mathrm{V}}_{\mathrm{C}}=\rho_{0}(\overline{\mathrm{U}}+\overline{\mathrm{W}})
$$

and we have the momentum integral

$$
\int_{S}\left[p \hat{n}+\rho_{0}(\bar{U}+\bar{W}) \cdot \hat{n}(\bar{U}+\bar{V})\right] d s=0
$$

This can be shown to be valid for any surface $\mathrm{S}$ enclosing a volume $\mathrm{V}$ of the fluid when the pressure is given by equation (3.10). After eliminating the pressure, the momentum integral in equation (3.24) becomes

$$
\rho_{0} \int_{S} \hat{\mathrm{n}} \cdot[-\mathrm{I}(\overline{\mathrm{U}} \cdot \overline{\mathrm{V}}+\overline{\mathrm{V}} \cdot \overline{\mathrm{W}} / 2)+(\overline{\mathrm{U}}+\overline{\mathrm{W}})(\overline{\mathrm{U}}+\overline{\mathrm{V}})] \mathrm{ds}
$$

where $I$ is the identity matrix for which $\hat{n} \cdot I=\hat{n}$. Applying the divergence theorem

$$
\int_{S} \hat{n} \cdot \bar{A} d s=\int_{V} \nabla \cdot \bar{A} d v
$$

replaces the surface integral by the volume integral

$$
\rho_{0} \int_{V}[-\nabla(\bar{U} \cdot \bar{V})-\nabla(\bar{V} \cdot \bar{W}) / 2+\bar{U} \cdot \nabla \bar{W}+\bar{W} \cdot \nabla \bar{V}+(\bar{U}+\bar{V}) \nabla \cdot \bar{W}] d v
$$


where we have made use of the fact that $\bar{U}$ is a constant vector and also

$$
\nabla \cdot(\mathrm{I} \psi)=\nabla \psi
$$

for a scalar $\psi$ and

$$
\nabla \cdot\left(\bar{V}_{1} \bar{V}_{2}\right)=\bar{V}_{2} \nabla \cdot \bar{V}_{1}+\bar{V}_{1} \cdot \nabla \bar{V}_{2}
$$

for vectors $\bar{V}_{1}$ and $\bar{V}_{2}$.

Now applying the formula from vector analysis

$$
\bar{v}_{1} \times\left(\bar{v}_{2} \times \bar{v}_{3}\right)=\left(\bar{v}_{1} \cdot \bar{v}_{3}\right) \bar{V}_{2}-\left(\bar{v}_{1} \cdot \bar{v}_{2}\right) \bar{v}_{3}
$$

to $\overline{\mathrm{W}} \times(\nabla \times \overline{\mathrm{V}})$ and noting that $\nabla$ operates only on $\overline{\mathrm{V}}$ yields

$$
\bar{W} \times(\nabla \times \bar{V})=\nabla[\bar{W}] \cdot \bar{V}-\bar{W} \cdot \nabla \bar{V}
$$

Since the vectors $\bar{V}$ and $\bar{W}$ are related by a constant symmetric matrix in the statement following equation (3.7) they have the property that

$$
\frac{\partial \bar{W}}{\partial x} \cdot \bar{V}=\bar{W} \cdot \frac{\partial \bar{V}}{\partial x}
$$

We finally obtain

$$
\overline{\mathrm{W}} \mathrm{x}(\nabla \times \overline{\mathrm{V}})=\nabla(\overline{\mathrm{V}} \cdot \overline{\mathrm{W}}) / 2-\overline{\mathrm{W}} \cdot \nabla \overline{\mathrm{V}}
$$

Similarly, as $\mathrm{U}$ is a constant vector, we have

$$
\overline{\mathrm{U}} \times(\nabla \times \bar{V})=\nabla(\overline{\mathrm{U}} \cdot \overline{\mathrm{V}})-\overline{\mathrm{U}} \cdot \nabla \overline{\mathrm{V}}
$$

With these substitutions, the volume integral finally becomes

$$
\rho_{0} \int_{V}[-\bar{U} \times(\nabla \times \bar{V})-\bar{W} \times(\nabla \times \bar{V})+(\bar{U} \cdot \bar{V}) \nabla \cdot \bar{W}] d v
$$

Since we assume potential flow, then $\nabla \times \bar{V}=0$ and $\nabla \cdot \bar{W}=0$. The integrand of the volume integral vanishes identically thereby proving that equation (3.24) holds for any arbitrary surface enclosing the flow when the pressure is given by equation (3.10). 
Let $S$ consist of a body $S_{b}$, both sides of a doublet sheet $S_{v}$, and a surface $S^{\prime}$ enclosing the body. It will be shown in section 3.7 that $\bar{W} \cdot \hat{n}$ is continuous across the doublet sheet.

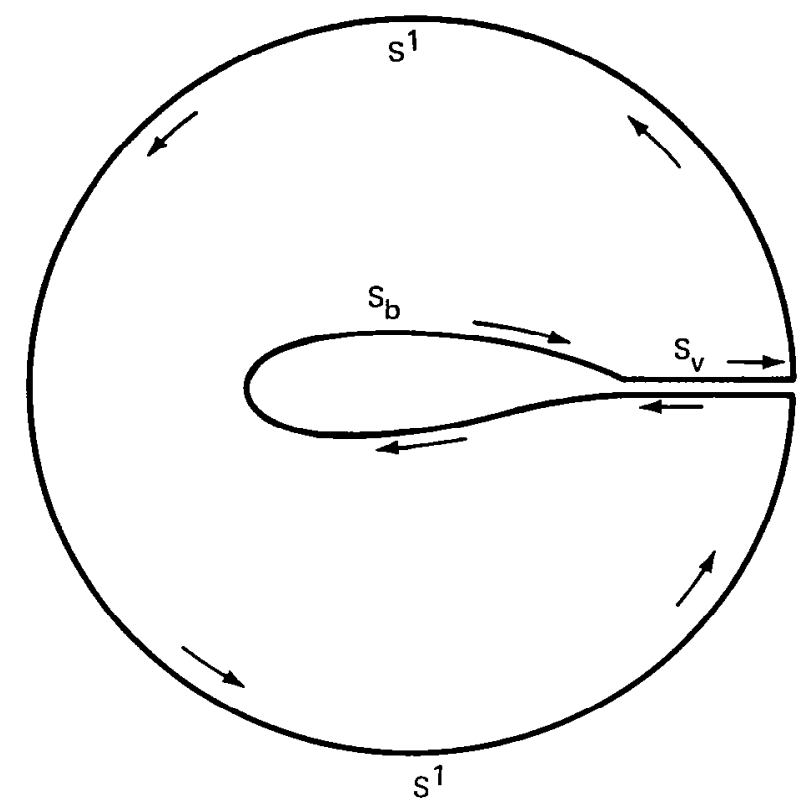

Cross-section of Surface $\mathbf{S}$

Since $(\overline{\mathrm{U}}+\overline{\mathrm{W}}) \cdot \hat{\mathrm{n}}=0$ on the body and $\overline{\mathrm{W}} \cdot \hat{\mathrm{n}}$ is continuous across the doublet sheet, the integral over $\mathrm{S}_{\mathrm{b}}$ and $\mathrm{S}_{\mathrm{v}}$ yields the body force

$$
\overline{\mathrm{F}}=\int_{\mathrm{S}_{\mathrm{b}}} \mathrm{p} \hat{\mathrm{n}} \mathrm{ds}
$$

Hence the body force $\Gamma$ can be found by an integral of the pressure and momentum through a surface $S^{\prime}$ enclosing the body using equation (3.24); namely,

$$
\bar{F}=-\int_{S^{\prime}}\left[p \hat{n}+\rho_{0}(\bar{U}+\bar{V})(\bar{U}+\bar{W}) \cdot \hat{n}\right] d s
$$

The surface $S^{\prime}$ is arbitrary but care must be taken in integrating about edges of the doublet sheets with singularities in the potential. For greater details the reader may consult Ward [10] and the applicable references. 


\subsection{INTEGRAL EQUATIONS FOR SUPERSONIC LINEARIZED FLOW}

Except for the uniqueness proof and scaling of the velocity potential, the foregoing analysis is directly applicable to supersonic flow. To derive an integral equation method we apply the divergence theorm to the quantity

$$
\phi_{1} \overline{\mathrm{W}}_{2}-\phi_{2} \overline{\mathrm{W}}_{1}
$$

where the subscripts 1 and 2 denote two distinct solutions of equation (3.7). Using the identity $\nabla \phi_{1} \cdot \mathrm{W}_{2}=\nabla \phi_{2} \cdot \mathrm{W}_{1}$, we obtain

$$
\int_{S}\left(\phi_{1} \overline{\mathrm{W}}_{2}-\phi_{2} \overline{\mathrm{W}}_{1}\right) \cdot \hat{\mathrm{n}} \mathrm{ds}=\int_{\mathrm{V}}\left(\phi_{1} \nabla \cdot \overline{\mathrm{W}}_{2}-\phi_{2} \nabla \cdot \overline{\mathrm{W}}_{1}\right) \mathrm{dv}
$$

For the subsonic method $\phi_{2}$ was chosen as the point source

$$
\phi_{2}=1 / \sqrt{\left(\mathrm{x}-\mathrm{x}_{0}\right)^{2}+\beta^{2} \mathrm{r}^{2}}, \quad \mathrm{r}^{2}=\left(\mathrm{y}-\mathrm{y}_{0}\right)^{2}+\left(\mathrm{z}-\mathrm{z}_{0}\right)^{2}
$$

which satisfies $\nabla \cdot \mathrm{W}_{2}=0$ except at the point $\mathrm{x}_{0}=\mathrm{x}, \mathrm{y}_{0}=\mathrm{y}$, and $\mathrm{z}_{0}=\mathrm{z}$. With $\phi_{2}$ from equation (3.27) in equalion (3.26), the surface integral yields a solution for $\phi_{1}$ in terms of source and doublet distributions over the surface. An analogous procedure was used by Hadamard [12], Ward [10], and by Heaslet and Lomax [13] for supersonic flow. Since $M>1$, the coefficient of the $\phi_{x x}$ term in equation (3.13) is negative and the differential equation becomes hyperbolic. The supersonic equivalent to equation (3.27) is then

$$
\phi_{2}=1 / R_{B}
$$

where

$$
R_{B}=\sqrt{\left(x-x_{0}\right)^{2}-B^{2} r^{2}} \text { and } B^{2}=M^{2}-1
$$

The supersonic case is much more complicated than the subsonic, since the equivalent point source has singularities along the surface $x-x_{0}= \pm B r$ as well as at the source point $x_{0}$, $\mathrm{y}_{0}, \mathrm{z}_{0}$. With $\phi_{2}$ substituted into equation (3.26) some of the integrals do not exist in the usual mathematical sense and require special treatment first introduced by Hadamard [12] and explained briefly farther on in this section of the report.

It is worthwhile to examine the fundamental differences between subsonic and supersonic flows. When a disturbance occurs at a time $t=0$ the wave propagates into the fluid at the speed of sound while at the same time the disturbance is being convected by the stream. In an infinite time with a subsonic free stream, the disturbance will fill entire space (see fig. 2). When the stream is supersonic, the disturbance is convected away from 
its origin at a speed faster than its propagation rate into the medium. Consequently, the disturbance is confined to a conical region formed by the envelope of spherical wave fronts of the convected disturbance source as shown in figure 3 . The sine of the cone angle is the ratio of sound speed $c_{0}$ to the free stream velocity $U_{0}$, or the reciprocal of the Mach number. The region of influence of the disturbance from a point is easily recognized from figure 4 as the region bounded by the downstream cone from $\left(x_{0}, y_{0}, z_{0}\right)$. In this region the radicand in the point source $\phi_{2}$ in equation (3.28) is positive or

$$
\left|\mathrm{x}-\mathrm{x}_{0}\right|>\mathrm{Br}
$$

The Mach cone has two nappes. The downstream cone $\mathrm{x}=\mathrm{x}_{0}+\mathrm{Br}$ bounds the domain of influence of the point $x_{0}, y_{0}, z_{0}$, while the upstream cone $x_{0}=x_{0}-B r$ bounds the region which influences the point $x_{0}, y_{0}, z_{0}$, or the domain of dependence of the point $\mathrm{x}_{0}, \mathrm{y}_{0}, \mathrm{z}_{0}$ (see fig. 4).

The Mach cone is also a characteristic surface associated with the differential equation. Across characteristic surfaces the normal derivative of the perturbation potential may be discontinuous while the tangential derivative remains continuous. This can occur only if the differential equation can be expressed in terms of derivatives along these surfaces. Characteristic surfaces are also the surfaces for which the normal component of the free stream velocity is equal to the speed of sound and hence can only exist in supersonic flow.

These characteristic surfaces can represent shocks in linearized supersonic flow since in the limit as shock strength approaches zero, the shock approaches the characteristic direction. For exact supersonic flow, entropy increases across a shock and the flow is usually no longer irrotational. Since the entropy is of the third order in shock strength, the assumption of irrotational flow is still valid to the second order in linearized supersonic flow. For flows containing only weak shocks, linearized theory can be expected to yield satisfactory results.

Discontinuous expansion waves also occur at characteristic surfaces in linearized theory. In exact theory, the expansion waves are in the form of a continuous centered fan of characteristics instead of a single discontinuity. The approximation of expansion waves by an 'expansion shock' is valid near the surface but is a poor representation of the flow at grcater distances away from the surface. Linearized theory solutions involving rapid expansions can be expected to yield good pressure distributions on the surface but the induced flow at large distances from the expansion surface will not be accurately described.

Because of the inverse square root singularity on the Mach cone of the supersonic source, the derivation of the integral equation method for subsonic flow using source and doublet surfaces cannot be carried over directly into supersonic flow, since derivatives of the source occur. Volterra (see ref. [14]) avoided this difficulty by using a different fundamental solution for $\phi_{2}$ which vanishes on the Mach cone. Hadamard [12] got around the difficulty by defining the finite part of the divergent integrals resulting from using the acoustic source equivalent to equation (3.28) in solutions of the wave aquation. This approach was also applied by Ward [10], Robinson [15], and Heaslet and Lomax [13] . To illustrate this concept, consider the following integral 


$$
I(\epsilon)=\int_{a+\epsilon}^{b} f(x) d x /(x-a)^{3 / 2}
$$

If $\mathrm{f}^{\prime}(\mathrm{a})$ exists and is bounded in the interval a to be, then $\mathrm{I}(\epsilon)$ exists. To assign a meaning for $\epsilon=0$ we write $I(\epsilon)$ in the form

$$
\begin{aligned}
& I(\epsilon)=\int_{a+\epsilon}^{b}[f(x)-f(a)] d x /(x-a)^{3 / 2}+f(a) \int_{a+\epsilon}^{b} d x /(x-a)^{3 / 2} \\
& =\int_{a+\epsilon}^{b}[f(x)-f(a)] d x /(x-a)^{3 / 2}-2 f(a) /(b-a)^{1 / 2}+2 f(a) / \epsilon^{1 / 2}
\end{aligned}
$$

As $\epsilon \rightarrow 0$ the first integral exists as an ordinary improper integral. We further write

$$
\begin{gathered}
I(\epsilon)=\int_{a}^{b}[f(x)-f(a)] /(x-a)^{3 / 2}-2 f(a) /(b-a)^{1 / 2}+2 f(a) / \epsilon 1 / 2 \\
\\
\quad-\int_{a}^{a+\epsilon}[f(x)-f(a)] d x /(x-a)^{3 / 2}
\end{gathered}
$$

Hadamard [12] defined the first two terms as the finite part of $I(0)$. Ward [10] used the notation *I to denote this finite part. Hence

$$
* I=\lim _{\epsilon \rightarrow 0}\left\{\mathrm{I}(\epsilon)-2 \mathrm{f}(\mathrm{a}) / \epsilon^{1 / 2}+0\left(\epsilon^{1 / 2}\right)\right\}
$$

The unique finite part of an infinite integral is demonstrated here for a single variable but it can be generalized to integration of several variables. The finite part can be shown to have the following propcrties (ref. [10]):

1. If I converges then * $\mathrm{I}=\mathrm{I}$.

2. The value of $* I$ is invariant under coordinate transformation provided the transformation itself is not singular on the surface of singularity of $\mathrm{I}$. 
3. If $* I$ involves vector quantities, then ${ }^{*} I$ is invariant under rotation of the coordinate axes.

4. If $\mathrm{I}(\epsilon)$ vanishes for all $\epsilon$, then $* I=0$.

5. Differentiation of finite part integrals with respect to a parameter may be moved inside the integral. For example,

$$
(\partial / \partial a) \int_{S}^{*} f d s=\int_{S}^{*} \partial f / \partial a d s
$$

Hadamard [12] also showed that the divergence theorem holds for the finite part integrals, i.e.,

$$
\int_{V}^{*} \nabla \cdot \bar{W} d v=\int_{S}^{*} \bar{W} \cdot \hat{n} d s
$$

and Robinson [15] extended the principle to include Stokes theorem

$$
\int_{S}^{*} \nabla x \bar{V} \cdot \hat{n} d s=\int_{c}^{*} \bar{V} \cdot d \bar{l}
$$

With the concept of finite part of an integral to give meaning to the divergent integrals, we are now able to proceed in the same manner as in incompressible flow. We first show that the mass flux from $\phi_{2}$ in equation (3.28), through a surface enclosing the point $x_{0}$, $y_{0}, z_{0}$ is independent of the choice of surface. We can assume without loss of generality, that $\mathrm{x}_{0}, \mathrm{y}_{0}, \mathrm{z}_{0}$ is the origin of coordinates. Let this surface contain the closed cylinder $-\mathrm{a} \leqslant \mathrm{X} \leqslant \mathrm{a}, \mathrm{r}^{2}=\mathrm{y}^{2}+\mathrm{z}^{2} \leqslant(\mathrm{a} / \mathrm{B})^{2}$, which intersects the downstream Mach cone at $\mathrm{x}=\mathrm{a}$. On this cylinder, $\phi_{2}$ and $\overline{\mathrm{W}}_{2}$ are non-zero only on the plane $\mathrm{x}=\mathrm{z}$.

To evaluate the mass flux we consider the surface $S(\epsilon)$ interior to the Mach cone as in figure 5 . Then the mass flux is given by

$$
\begin{aligned}
\mathrm{J}(\epsilon)= & \int_{\mathrm{S}^{(\epsilon)}} \overline{\mathrm{W}} \cdot \hat{\mathrm{n}} \mathrm{d} s=-\mathrm{B} \int_{\mathrm{S}^{(\epsilon)}} \partial \phi_{2} / \partial \mathrm{x} \quad \mathrm{dydz}=\int_{\mathrm{S}^{(\epsilon)}} \mathrm{B}^{2} \mathrm{adydz} / \sqrt{\left(\mathrm{a}^{2}-\mathrm{B}^{2} \mathrm{r}^{2}\right)^{3}} \\
& =\int_{0}^{2 \pi} \int_{0}^{\mathrm{B} \eta / \mathrm{a}} \rho \mathrm{d} \rho \mathrm{d} \theta / \sqrt{\left(1-\rho^{2}\right)^{3}}=2 \pi\left[1 / \sqrt{1-\mathrm{B}^{2} \eta^{2} / \mathrm{a}^{2}}-1\right]
\end{aligned}
$$


Since $\eta=\mathrm{a} / \mathrm{B}-\epsilon \frac{\sqrt{1+\mathrm{B}^{2}}}{\mathrm{~B}}$

$$
J(\epsilon)=-2 \pi+2 \pi \sqrt{\frac{a}{1+B^{2}}} \frac{1}{\epsilon^{1 / 2}}
$$

and

$$
\mathrm{J} *=\lim _{\epsilon \rightarrow 0}\left[\mathrm{~J}(\epsilon)-2 \pi / \epsilon^{1 / 2} \sqrt{\mathrm{a} /\left(1+\mathrm{B}^{2}\right)}\right]=-2 \pi
$$

This gives the mass flux from the plane cutting the cone. We need to show that for two surfaces, $S_{1}$ and $S_{2}$, which cut the cone for $x>0$ as shown in figure 5 , the mass flux through each surface is the same, or

$$
\int_{S_{1}}^{*} w \cdot \hat{n} d s=\int_{S_{2}}^{*} w \cdot \hat{n} d s
$$

Applying the divergence theorem to the volume between the two surfaces yields

$$
\int_{S_{1}}^{*} \bar{W} \cdot \hat{n} d s=\int_{S_{2}}^{*} \bar{W} \cdot \hat{n} d s=\int_{V}^{*} \nabla \cdot \bar{W} d s
$$

where $\mathrm{V}$ is the volume enclosed by the two surfaces and the Mach cone. Since this volume does not contain the origin where $\bar{W}$ is not defined, $\nabla \cdot \bar{W}=0$ from equation (3.7) and the mass flux is seen to be independent of the choice of surface enclosing the source point. The fundamental solution

$$
\phi=-1 / 2 \pi R_{B}
$$

then represents a true supersonic source of unit strength.

\subsection{INTEGRAL EQUATION FOR SUPERINCLINED PANELS}

We now derive an integral equation for superinclined surfaces, i.e., surfaces inclined with the free stream at angles greater than the Mach angle. We consider the volume in figure 6 enclosed by the surface $S$, the upstream Mach cone $\Gamma$ and the circle $S_{1}$, near to but excluding the vertex of the cone at the point $x, y, z$. For $\phi_{2}$ in equation (3.26) we use 


$$
\begin{aligned}
\phi_{2} & =1 / R_{B} \text { for } R_{B} 2>0, x_{0}<x \\
& =0 \text { at all other points }
\end{aligned}
$$

and for $\phi_{1}$, we simply write $\phi$. Since by equation (3.7) $\nabla \cdot \overline{\mathrm{W}}=\nabla \cdot \overline{\mathrm{W}}_{2}=0$, equation (3.26) yields

$$
\int_{S+\Gamma+S_{1}}^{*}\left[\phi \overline{\mathrm{W}}_{2}-\phi_{2} \overline{\mathrm{W}}\right] \cdot \hat{\mathrm{n}} \mathrm{ds}=0
$$

The integration over the cone surface $\Gamma$ can be eliminated since the integrand is singular there and, hence, it makes no contribution to the finite part. The integral over the small circle $S_{1}$ involving $\bar{W}_{2}$ becomes

$$
\int_{S_{1}}^{*} \phi \bar{W}_{2} \cdot \hat{n} \mathrm{ds} \simeq \tilde{\phi} \int_{S_{1}}^{*} \bar{W}_{2} \cdot \hat{n} d s
$$

where the tilda denotes average value over the surface $S_{1}$. This is the same in tegral used for computing the mass flux from a point source, but with the normal directed in the opposite direction. Hence, we obtain, by letting the surface $S_{1}$ approach the cone vertex,

$$
\mathrm{S}_{1} \underset{0}{\operatorname{limit}} \int_{\mathrm{S}_{1}} \phi \overline{\mathrm{W}}_{2} \cdot \hat{\mathrm{n} d \mathrm{ds}}=2 \pi \phi(\mathrm{x}, \mathrm{y}, \mathrm{z})
$$

Similarly, for the other integral over $\mathrm{S}_{1}$

$$
\begin{gathered}
\int_{\mathrm{S}_{1}} \phi_{2} \overline{\mathrm{W}} \cdot \hat{\mathrm{n}} \mathrm{ds} \simeq \overline{\mathrm{W}} \cdot \hat{\mathrm{n}} \int_{\mathrm{S}_{1}} \phi_{2} \mathrm{ds}=\overline{\mathrm{W}} \cdot \hat{\mathrm{n}} \int_{0}^{2 \pi} \int_{0}^{\left(\mathrm{x}-\mathrm{x}_{0}\right) / \mathrm{B}} \frac{\mathrm{rdrd \theta}}{\sqrt{\left(\mathrm{x}-\mathrm{x}_{0}\right)^{2}-\mathrm{B}^{2} \mathrm{r}^{2}}} \\
=2 \pi \frac{\overline{\mathrm{W}} \cdot \hat{\mathrm{n}}\left(\mathrm{x}-\mathrm{x}_{0}\right)}{\mathrm{B}} \int_{0}^{1} \frac{\rho \mathrm{d} \rho}{\sqrt{1-\rho^{2}}}=2 \pi\left(\mathrm{x}-\mathrm{x}_{0}\right) \overline{\mathrm{W}} \cdot \hat{\mathrm{n}} / \mathrm{B}
\end{gathered}
$$


The contribution of $S_{1}$ from $\phi_{2}$ vanishes as $x-x_{0}$ goes to zero. Finally, the integral equation for superinclined surfaces takes the form

$$
\phi=\frac{1}{2 \pi} \int\left[\overline{\mathrm{W}} \cdot \hat{\mathrm{n}} \phi_{2}-\phi \overline{\mathrm{W}}_{2} \cdot \hat{\mathrm{n}}\right] \mathrm{ds}
$$

After substituting $\phi_{2}$ and $\overline{\mathrm{W}}_{2}$,

$$
\phi(\mathrm{R})=\frac{1}{2 \pi} \int_{\mathrm{S}} \frac{\overline{\mathrm{W}} \cdot \hat{\mathrm{n}} \mathrm{ds}}{\mathrm{R}_{\mathrm{B}}}-\frac{\mathrm{B}^{2}}{2 \pi} \int_{\mathrm{S}}^{*} \frac{\phi\left(\overline{\mathrm{R}}_{0}-\overline{\mathrm{R}}\right) \cdot \hat{\mathrm{n}} \mathrm{ds}}{\mathrm{R}_{\mathrm{B}}{ }^{3}}
$$

It is not necessary to take the finite part of the first integral since the integrand has an integrable singularity. Since

$$
\overline{\mathrm{W}}_{2}=\hat{\mathrm{n}} \cdot\left(-\mathrm{B}^{2} \frac{\partial}{\partial \mathrm{x}_{0}}, \frac{\partial}{\partial \mathrm{y}_{0}}, \frac{\partial}{\partial \mathrm{z}_{0}}\right)\left(\frac{1}{\mathrm{R}_{\mathrm{B}}}\right)=\frac{\mathrm{B}^{2}\left(\overline{\mathrm{R}}_{0}-\overline{\mathrm{R}}\right) \cdot \hat{\mathrm{n}}}{\mathrm{R}_{\mathrm{B}}{ }^{3}}
$$

the first integral of equation (3.31) represents a distribution of sources while the second integral a distribution of doublets. It is easy to show that $\phi$ takes on the prescribed values on the surface $S$. As the point $x, y, z$ moves to the surface, the integration over $S$ becomes the same as the previous integration over $S_{1}$ near the vertex of the Mach cone, but with the normal now directed inwards toward the vertex. Thus,

$$
\phi=\frac{1}{2 \pi} \int_{\mathrm{S}}\left(\overline{\mathrm{W}} \cdot \hat{\mathrm{n}} \phi_{2}-\phi \overline{\mathrm{W}}_{2} \cdot \hat{\mathrm{n}}\right) \mathrm{ds} \simeq \frac{1}{2 \pi} \overline{\mathrm{W}} \cdot \hat{\mathrm{n}} \int_{\mathrm{S}_{1}} \phi_{2} \mathrm{ds}-\frac{1}{2 \pi} \phi \int_{\mathrm{S}_{1}} \overline{\mathrm{W}}_{2} \cdot \mathrm{nds}=\phi
$$

Similarly, $\overline{\mathrm{W}} \cdot \hat{\mathrm{n}}$ can be shown to take on the assigned value.

\subsection{UNIQUENESS OF THE SOLUTIONS WITH POTENTIAL AND LINEAR MASS FLUX BOUNDARY CONDITIONS ON SUPERINCLINED SURFACES}

It is possible to show that when $\phi$ and $\bar{W} \cdot \hat{n}$ are prescribed on a surface inclined at an angle greater than the Mach angle, that the solution is unique. To that end, we apply the divergence theorem to the matrix quantity

$$
\overline{\mathrm{V}} \overline{\mathrm{W}}-(\overline{\mathrm{V}} \cdot \overline{\mathrm{W}}) \mathrm{I} / 2
$$


where I is the identity matrix. We obtain

$$
\int_{V}\{\bar{V} \nabla \cdot \bar{W}+\bar{W} \cdot \nabla \bar{V}-\nabla(\bar{V} \cdot \bar{W}) / 2\} d v=\int_{S}(\bar{V} \bar{W} \cdot \hat{n}-\bar{V} \cdot \bar{W} \hat{n} / 2) d s
$$

where we have used

$$
\nabla \cdot(\bar{V} \bar{W}) \equiv \bar{W} \nabla \cdot \bar{V}+\bar{V} \cdot \nabla W \text { and } \nabla \cdot(\bar{V} \cdot \bar{W}) I=\nabla(\bar{V} \cdot \bar{W})
$$

For irrotational and source free flow, the volume integrals disappear since we showed in section 3.3 that $\bar{W} \cdot \nabla \bar{V}-\nabla(\bar{V} \cdot \bar{W}) / 2=\bar{W} \times \nabla \times \bar{V}=0$. Consider the $x$ component of this surface integral:

$$
\int_{\mathrm{S}}\left[\phi_{\mathrm{x}}\left(\phi_{\mathrm{y}} \mathrm{n}_{\mathrm{y}}+\mathrm{n}_{\mathrm{z}} \phi_{\mathrm{z}}-\mathrm{B}^{2} \phi_{\mathrm{x}} \mathrm{n}_{\mathrm{x}}\right)-\left(-\mathrm{B}^{2} \phi_{\mathrm{x}}^{2}+\phi_{\mathrm{y}}^{2}+\phi_{\mathrm{z}}^{2}\right) \mathrm{n}_{\mathrm{x}} / 2\right] \mathrm{ds}=0
$$

Simplifying and factoring yields

$$
-\int_{\mathrm{S}}\left[\left(\phi_{\mathrm{y}}-\phi_{\mathrm{x}} \mathrm{n}_{\mathrm{y}} / \mathrm{n}_{\mathrm{x}}\right)^{2}+\left(\phi_{\mathrm{z}}-\phi_{\mathrm{x}} \mathrm{n}_{\mathrm{z}} / \mathrm{n}_{\mathrm{x}}\right)^{2}+\left(\mathrm{B}^{2}-\frac{\mathrm{n}_{\mathrm{y}}^{2}+\mathrm{n}_{\mathrm{z}}^{2}}{\mathrm{n}_{\mathrm{x}}^{2}}\right) \phi_{\mathrm{x}}{ }^{2}\right] \mathrm{n}_{\mathrm{x}} \mathrm{ds}=0
$$

Denote by $\phi_{\mathrm{S}}$ the boundary values of $\phi$ and its derivatives. Then the squared terms in the first two pairs of parentheses are $\phi_{\mathrm{S}}{ }^{2} \mathrm{y}$ and $\phi_{\mathrm{S}}{ }^{2} \mathrm{z}$, respectively. Furthermore, if $\Gamma$, say, is a characteristic surface, then

$$
\mathrm{B}^{2}-\left(\mathrm{n}_{\mathrm{y}}^{2}+\mathrm{n}_{\mathrm{z}}^{2}\right) / \mathrm{n}_{\mathrm{x}}^{2}=0
$$

On such a characteristic surface $\Gamma$, the integral becomes

$$
-\operatorname{Sign}\left(n_{x}\right) \int_{\Gamma}\left[\phi_{s y}^{2}+\phi_{s z}^{2}\right] d y d z
$$


Let $\mathrm{S}$ consist of three parts as shown in figure 7.

1. The characteristic cone $x=x_{1}-B \sqrt{\left(y-y_{1}\right)^{2}+\left(z-z_{1}\right)^{2}}$ denoted by $\Gamma$.

2. The plane $x=0$ with $B^{2}\left[\left(y-y_{1}\right)^{2}+\left(z-z_{1}\right)^{2}\right]<x_{1}{ }^{2}$ denoted by $S_{1}$, and on which boundary conditions are to be applied.

3. The plane $\mathrm{x}=\mathrm{x}_{0}, 0<\mathrm{x}_{0}<\mathrm{x}_{1}$, with $\mathrm{B}^{2}\left[\left(\mathrm{y}-\mathrm{y}_{1}\right)^{2}+\left(\mathrm{z}-\mathrm{z}_{1}\right)^{2}\right]<\left(\mathrm{x}_{0}-\mathrm{x}_{1}\right)^{2}$ denoted by $S_{3}$.

Since on $S_{1}$ and $S_{3}$, we have $n_{x}=-1$ and 1 , respectively, with $n_{y}=n_{z}=0$, then the integral in equation (3.34) becomes

$$
\begin{gathered}
-\iint_{\Gamma}\left(\phi_{\mathrm{sy}}^{2}+\phi_{\mathrm{sz}}^{2}\right) \mathrm{dydz}+\int_{\mathrm{S}_{1}} \int\left[\mathrm{B}^{2} \phi_{\mathrm{x}}{ }^{2}+\phi_{\mathrm{y}}{ }^{2}+\phi_{\mathrm{z}}^{2} \mathrm{dyd}\right] \\
-\int_{\mathrm{S}_{3}} \int_{\mathrm{B}}\left[\mathrm{B}^{2} \phi_{\mathrm{x}}{ }^{2}+\phi_{\mathrm{y}}{ }^{2}+\phi_{\mathrm{y}}{ }^{2}+\phi_{\mathrm{z}}{ }^{2}\right] \mathrm{dydz}=0
\end{gathered}
$$

since on $\Gamma, \mathrm{n}_{\mathrm{X}}>0$.

Let $\phi=\phi_{1}-\phi_{2}$ where the subscripts 1 and 2 denote two solutions with the same prescribed values of $\phi$ and $\phi_{\mathrm{X}}$ on the plane $\mathrm{x}=0$, i.e., on the surface $\mathrm{S}_{1}$. Then $\phi=\phi_{\mathrm{X}}=0$ on $S_{1}$ and the second integral over $S_{1}$ vanishes in equation (3.35). It follows from the form of the remaining integrals that

$$
\phi_{\mathrm{sy}}=\phi_{\mathrm{Sz}}=\phi_{\mathrm{x}}=\phi_{\mathrm{y}}=\phi_{\mathrm{z}}=0
$$

The surface $S_{3}$ may be varied arbitrarily along the cone axis. Hence, $\phi_{\mathrm{X}}=\phi_{\mathrm{y}}=\phi_{\mathrm{Z}}=0$ everywhere inside the cone. Therefore, when $\phi_{\mathrm{X}}$ and $\phi$ are prescribed on $\mathrm{S}_{1}$, then $\phi$ is uniquely determined inside the Mach cone from the point $\mathrm{x}_{1}, \mathrm{y}_{1}, \mathrm{z}_{1}$ in figure 7.

Under the section on coordinate transformations, the hyperbolic distance and the differential equation are shown to be invariant under a rotation about the $\mathrm{x}$ axis and under an oblique transformation in the plane containing the $\mathrm{x}$ axis. If the transformation is chosen for a superinclined panel so that the new variables $y, z$ are in the plane of the panel and $\mathrm{x}$ is the variable out of the panel; then, for panels inclined to the free stream at angles greater than the Mach angle, the area element and the conormal derivative transform according to

$$
\left(\partial \phi / \partial \mathrm{n}_{\mathrm{c}}\right) \mathrm{ds} \rightarrow(\partial \phi / \partial \mathrm{x}) \mathrm{dzdy}
$$


Ward [10] states that for superinclined surfaces, the conditions

$$
\phi, \partial \phi / \partial \mathrm{x}
$$

are generalized to $\phi$ and $\partial \phi / \partial$ n. The boundary conditions should more correctly be generalized to

$$
\phi, \partial \phi / \partial \mathrm{n}_{\mathrm{c}} \text { or } \overline{\mathrm{w}} \cdot \hat{\mathrm{n}}
$$

prescribed on the panel.

\subsection{INTEGRAL EQUATION FOR SUBINCLINED PANELS}

To apply panel methods for the solution of the supersonic flow over wings and bodies, we must consider subinclincd surfaccs, i.c., surfaces inclined at angles less than the Mach angle. In section 3.6, it was shown that boundary conditions can only be applied on the downwind side of the superinclined surface. For the subinclined surface, boundary conditions may be applied to either or both sides. Consider an almost planar surface. If the surface is slender and lies within the Mach cone, then the perturbed flow is confined to the downstream Mach cone emanating from its vertex. If the surface cuts the downstream Mach cone emanating from the forwardmost point of the surface, then the region of disturbances lies downstream of the characteristic surface formed by the envelope of Mach cones emanating from the leading edge (see fig. 8). This is the surface at which the flow first experiences a disturbance. To apply equation (3.26) we consider 2 volumes, $V_{1}$ and $\mathrm{V}_{2}$, illustrated by the cross section drawing in figure 8 . The volume $\mathrm{V}_{1}$ is bounded by the following surfaces:

$\Gamma_{1}$ consisting of the leading edge characteristic surface cut out by the Mach cone $\Gamma$ from the point $x, y, z$.

$S_{\epsilon}$ the circle of radius $\epsilon$ formed by the plane excluding the highest order singularity at $x, y, z$ from the volume $V$.

$\mathrm{S}_{\mathrm{w}}$ the portion of the subinclined surface cut off by the Mach cone where boundary conditions are to be applied.

We have already shown in equation (3.30) that as $\epsilon \rightarrow 0$ the contribution to the surface integral $S_{\epsilon}$ was equal to

$$
2 \pi \phi(\mathrm{x}, \mathrm{y}, \mathrm{z})
$$

The direction of the conormal along a Mach cone or any characteristic surface lies along the generator and hence $\bar{W} \cdot \hat{n}$ is the perturbation tangential velocity. Because $\bar{W} \cdot \hat{n}$ is zero ahead of the characteristic surface $\Gamma_{1}$ then $\bar{W} \cdot \hat{n}$ is zero on the downstream side since this is required by the continuity of mass and momentum across the surface. Hence, $\phi$ is constant on the characteristic surface $\Gamma_{1}$. Since the addition of a constant to the velocity potential does not affect the velocity, we may choose this constant as zero to eliminate 
the integral over the surface $\Gamma_{1}$. Since the finite part cancels the integral over the Mach cone $\Gamma$ where the integrand is infinite, we obtain finally from equation (3.26).

$$
\phi(x, y, z)=-\frac{1}{2 \pi} \int_{S_{\mathrm{w}}}^{*}\left(\phi^{+} \overline{\mathrm{W}}_{2}-\overline{\mathrm{W}}^{+} \phi_{2}\right) \cdot \hat{\mathrm{n} d \mathrm{~d}}
$$

in the notation of Ward [10] this becomes

$$
\phi(\overline{\mathrm{R}})=\frac{1}{2 \pi} \int_{\mathrm{S}_{\mathrm{W}}} \frac{\overline{\mathrm{W}}^{+}\left(\overline{\mathrm{R}}_{0}\right) \cdot \hat{\mathrm{n} d s}}{\mathrm{R}_{\mathrm{B}}}-\frac{\mathrm{B}^{2}}{2 \pi} \int_{\mathrm{S}_{\mathrm{w}}} \frac{\phi^{+}\left(\overline{\mathrm{R}}_{0}\right)\left(\overline{\mathrm{R}}_{0}-\overline{\mathrm{R}}\right) \cdot \hat{\mathrm{n}} \mathrm{ds}}{\mathrm{R}_{\mathrm{B}}{ }^{3}}
$$

or by defining the conormal vector, $\bar{n}_{c}=\left(-\stackrel{2}{2} n_{x}, n_{y}, n_{z}\right)$, we also obtain

$$
\phi(\overline{\mathrm{R}})=\frac{1}{2 \pi} \int_{\mathrm{S}_{\mathrm{W}}} \frac{\overline{\mathrm{W}}^{+}\left(\overline{\mathrm{R}}_{0}\right) \cdot \hat{\mathrm{n} d s}}{\mathrm{R}_{\mathrm{B}}}-\frac{1}{2 \pi} \int_{\mathrm{S}_{\mathrm{W}}}^{*} \phi^{+}\left(\overline{\mathrm{R}}_{0}\right) \frac{\partial}{\partial \mathrm{n}_{\mathrm{c}}}\left(\frac{1}{\mathrm{R}_{\mathrm{B}}}\right) \mathrm{ds}
$$

Comparing this equation with equation (3.31) we see that this formula holds whatever the inclination of the surface $S_{W}$. For superinclined surfaces, we showed in section 3.6 that $\phi$ and $\overline{\mathrm{W}} \cdot \hat{\mathrm{n}}$ take on the boundary values $\phi^{+}$and $\overline{\mathrm{W}}^{+} . \hat{\mathrm{n}}$ in the integrals on the right hand side of the equation. For subinclined panel surfaces, $\phi$ and $\overline{\mathrm{W}} \cdot \hat{\mathrm{n}}$ are not independent. We now consider the volume $\mathrm{V}_{2}$ which is bounded by the surfaces consisting of the portion of the Mach cone $\Gamma$ on the side of $S_{W}$ opposite the point $x, y, z$ and the surface $\Gamma_{2}$ of the lower characteristic surface from the leading edge. Since for the finite part of the integral, the integrals over $\Gamma_{2}$ and $\Gamma$ vanish where the integrands are infinite, we obtain

$$
0=-\frac{1}{2 \pi} \int_{S_{W}}^{*}\left(\phi-\bar{W}_{2}-\bar{W}^{-} \phi_{2}\right) \cdot \hat{n} \mathrm{ds}
$$

or

$$
0=-\frac{1}{2 \pi} \int \frac{\bar{W}^{-}\left(\bar{R}_{0}\right)}{R_{B}} \cdot \frac{\hat{n}}{d s}-\frac{B^{2}}{2 \pi} \int_{S_{W}}^{*} \frac{\phi^{-}\left(\bar{R}_{0}\right)\left(\bar{R}_{0}-\bar{R}\right) \cdot \hat{n} d s}{R_{B}{ }^{3}}
$$


This indicates the dependence of $\phi$ and $\bar{W} \cdot \hat{n}$ boundary conditions on $S_{W}$ for subinclined surfaces. This is the same surface as in equation (3.39) but $\hat{n}$ is directed outward on the opposite side of $S_{W}$. Adding equations (3.39) and (3.40) then yields

$$
\phi(\overline{\mathrm{R}})=\frac{1}{2 \pi} \int_{\mathrm{S}_{\mathrm{W}}} \frac{\left[\overline{\mathrm{W}}^{+}\left(\overline{\mathrm{R}}_{0}\right)-\overline{\mathrm{W}}^{-}\left(\overline{\mathrm{R}}_{0}\right)\right] \cdot \hat{\mathrm{n}} \mathrm{ds}}{\mathrm{R}_{\mathrm{B}}}-\frac{\mathrm{B} 2}{2 \pi} \int_{\mathrm{S}_{\mathrm{W}}}^{*} \frac{\left[\phi^{+}\left(\overline{\mathrm{R}}_{0}\right)-\phi-\left(\overline{\mathrm{R}}_{0}\right)\right]\left(\overline{\mathrm{R}}_{0}-\overline{\mathrm{R}}, \cdot \hat{\mathrm{n} d s}\right.}{\mathrm{R}_{\mathrm{B}}{ }^{3}}
$$

Although equation (3.41) was derived with the point $\mathrm{R}$ in the upper region of figure 8, it is valid for a point anywhere in the flow field. We can deduce from equation (3.41) that a discontinuity in the perturbation mass flux across a surface with the perturbation potential itself continuous, produces a distribution of sources of strength

$$
\sigma=\mathrm{W}^{+} \cdot \hat{\mathrm{n}}-\overline{\mathrm{W}}^{-} \cdot \hat{\mathrm{n}}
$$

with the flow field described by the potential

$$
\phi=\frac{1}{2 \pi} \int_{\mathrm{S}_{\mathrm{W}}} \sigma \frac{\mathrm{ds}}{\mathrm{R}_{\mathrm{B}}}
$$

Similarly, a jump in the velocity potential across a surface with continuous normal mass flux produces a doublet sheet of strength

$$
\mu=\phi^{+}\left(\overline{\mathrm{R}}_{0}\right)-\phi^{-}\left(\overline{\mathrm{R}}_{0}\right)
$$

whose flow field is described by the potential

$$
\phi=-\frac{\mathrm{B}^{2}}{2 \pi} \int_{\mathrm{S}_{\mathrm{W}}}^{*} \frac{\mu\left(\overline{\mathrm{R}}_{0}\right)\left(\overline{\mathrm{R}}_{0}-\overline{\mathrm{R}}\right) \cdot \hat{\mathrm{n}} \mathrm{ds}}{\mathrm{R}_{\mathrm{B}}{ }^{3}}=-\frac{1}{2 \pi} \int^{*} \mu\left(\overline{\mathrm{R}}_{0}\right) \frac{\partial}{\partial \mathrm{n}_{\mathrm{c}}}\left(\frac{1}{\mathrm{R}_{\mathrm{B}}}\right) \mathrm{ds}
$$

Conversely, a source distribution $\sigma$ defined over a surface produces a jump in the normal component of perturbation mass flux of magnitude $\sigma$ and continuous potential across the surface while a doublet distribution produces a discontinuity in potential across the surface with the normal mass flux remaining continuous. 


\subsection{UNIQUENESS OF SOLUTION ON SUBINCLINED SURFACES}

Proving uniqueness for certain boundary conditions applied to subinclined surfaces is not as straightforward as for the superinclined surfaces. Consider the following integral derived in Appendix 1 of Ward [10].

$$
\int_{S}\left(\bar{U} \cdot \bar{V} \bar{W} \cdot \hat{n}-\frac{1}{2} \bar{V} \cdot \bar{W} \bar{U} \cdot \hat{n}\right) d s=\int_{V} \bar{U} \cdot[\bar{V} \nabla \cdot \bar{W}-\bar{W} \times \nabla \times \bar{V}] d v
$$

The volume integral becomes zero for irrotational flow in which

$$
\nabla \cdot \overline{\mathrm{W}}=\nabla \times \overline{\mathrm{V}}=0
$$

We choose the surface $S$ to consist of the threc surfaces in figure 9 or

1. Both sides of the wing surface $S_{W}$.

2. A plane downstream of wing leading edges $S_{3}$ and normal to the free stream $\bar{U}$.

3. The characteristic surface $\Gamma_{1}$ from the leading edge of the surface $S_{W}$.

Assume $\overline{\mathrm{U}}$ to be in the $\mathrm{x}$ direction. Then the integral over $\mathrm{S}_{3}$ becomes

$$
\frac{U_{0}}{2} \int_{S_{3}}\left(B^{2} u^{2}+v^{2}+w^{2}\right) d y d z
$$

The integral over $\Gamma_{1}$ vanishes since the integrand was proved by Ward to be continuous across characteristic surfaces and $\bar{W}=\bar{V}=0$ ahead of the surface. Equation (3.44) becomes

$$
\begin{aligned}
& \frac{U_{0}}{2} \int_{S_{3}}\left(B^{2} u^{2}+v^{2}+w^{2}\right) d y d z+\int_{S_{W}}\left(\bar{U} \cdot \bar{V}^{+} \bar{W}^{+} \cdot \hat{n}-\frac{1}{2} \bar{V}^{+} \cdot \bar{W}^{+} \bar{U} \cdot \hat{n}\right) d s \\
& \quad+\int_{S_{W}} \bar{U} \cdot \bar{V}^{-} \bar{W}^{-} \cdot \hat{n}-\frac{1}{2} \bar{V}^{-} \cdot \bar{W}^{-} \cdot \hat{n} \quad d s=0
\end{aligned}
$$

To express the integrand in a form more suitable for considering normal mass flux and tangential velocity boundary conditions, we divide $\bar{V}$ and $\overline{\mathrm{W}}$ into normal and tangential components. 


$$
\begin{aligned}
& \bar{V}=(\bar{V} \cdot \hat{n}) \hat{n}+\hat{n} \times(\bar{N}+\hat{n})=(\bar{V} \cdot \hat{n}) \hat{n}+\bar{V}_{t} \\
& \bar{W}=(\bar{W} \cdot \hat{n}) \hat{n}+\hat{n} \times(\bar{W} \times \hat{n})=(\bar{W} \cdot \hat{n}) \hat{n}+\bar{W}_{t}
\end{aligned}
$$

Then the integrand takes the form

$$
\bar{U} \cdot \bar{V} \bar{W} \cdot \hat{n}-\bar{V} \cdot \bar{W} \bar{U} \cdot \hat{n} / 2=\left[\bar{U} \cdot \hat{n} \bar{V} \cdot \hat{n} / 2+\bar{U} \cdot \bar{V}_{t}\right] \bar{W} \cdot \hat{n}-\bar{w}_{t} \cdot \bar{V}_{t} \bar{U} \cdot \hat{n} / 2
$$

and equation (3.45) becomes

$$
\begin{aligned}
& \left(U_{0} / 2\right) \int_{S_{3}}\left(B^{2} u^{2}+v^{2}+w^{2}\right) d y d z+\int_{S_{W}}\left\{\left[\frac{1}{2} \bar{U} \cdot \hat{n} \bar{V}^{+} \cdot \hat{n}+\bar{U} \cdot \bar{V}_{t}^{+}\right] \bar{W}^{+} \cdot \hat{n}\right. \\
& \left.\quad-\frac{1}{2} \bar{V}_{t}^{+} \cdot \bar{W}_{t}^{+} \bar{U} \cdot \hat{n}\right\} d s+\int_{S_{W}}\left\{\left[\frac{1}{2} \bar{U} \cdot \hat{n} \bar{V}^{-} \cdot \hat{n}+\bar{U} \cdot \bar{V}_{t}^{-}\right] \bar{W}^{-} \cdot \hat{n}\right. \\
& \left.-\frac{1}{2} \bar{V}_{t}^{-} \cdot \bar{W}_{t}^{-} \bar{U} \cdot \hat{n}\right\} d s
\end{aligned}
$$

The approach to proving uniqueness using the preceding integrals is similar to that used for the superinclined panels. The vectors $\bar{V}$ and $\bar{W}$ are chosen as the difference between two solutions which apply the same boundary conditions to the surface $S_{W}$. If the surface integrals over $S_{w}$ are all zero, then the integral over $S_{3}$ is zero. Since the integral over $S_{3}$ is the sum of squares, the integrand and the individual velocity components must also be zero. Since $S_{3}$ is a general surface and may be moved, then $u=v=w=0$ and the two solutions are identical.

From the form of the integrals above, it appears that if both $\bar{W} \cdot \hat{n}$ and $\bar{V}_{t}$ boundary conditions are applied to each surface, the solution is unique. However, for three dimensional configurations, we usually are interested in solutions for the flow over the exterior (or + side) of the surface. We then eliminate the interior flow by setting

$$
\overline{\mathrm{W}}^{-} \cdot \hat{\mathrm{n}} \equiv \overline{\mathrm{V}}^{-} \times \hat{\mathrm{n}}=0
$$

Since we demonstrated in section 3.7 that the source sheet produces a jump only in the normal mass flux and the doublet sheet, a jump only in the tangential velocity, we have on the upper surface 


$$
\begin{aligned}
& \overline{\mathrm{W}}^{+} \cdot \hat{\mathrm{n}}=\sigma \\
& \overline{\mathrm{V}}^{+} \times \hat{\mathrm{n}}=\nabla \mu \times \hat{\mathrm{n}}
\end{aligned}
$$

where $\sigma$ and $\mu$ are the source and doublet distributions on the surface, respectively. Subject to equation (3.47), two solutions having the same source distribution and doublet distribution can be seen from equations (3.46) to be identical. We cannot choose both $\sigma$ and $\mu$ independently, however, since we must also have, from equation (3.47),

$$
\hat{\mathrm{n}} \times \overline{\mathrm{V}}^{-}=\hat{\mathrm{n}} \times \nabla \phi^{-}=0
$$

Since this is the tangential derivative on the surface, it is identically satisfied when

$$
\phi^{-}=0
$$

or from equation (3.41), (3.42), and (3.43),

$$
\frac{\mathrm{B}^{2}}{2 \pi} \int_{\mathrm{S}_{\mathrm{W}}}^{*} \frac{\mu\left(\overline{\mathrm{R}}_{0}\right)\left(\overline{\mathrm{R}}_{0}-\overline{\mathrm{R}}\right) \cdot \hat{\mathrm{ndd}}}{\mathrm{R}_{\mathrm{B}}{ }^{3}}=\frac{1}{2 \pi} \int_{\mathrm{s}_{\mathrm{W}}} \frac{\sigma \mathrm{ds}}{\mathrm{R}_{\mathrm{B}}}
$$

Hence, we are free to choose only one of the functions $\sigma$ and $\mu$. The most generally applied boundary condition is the condition that no mass flux penetrate the surface or

$$
\overline{\mathrm{W}}^{+} \cdot \hat{\mathrm{n}}=-\overline{\mathrm{U}} \cdot \hat{\mathrm{n}}
$$

which fixes the value of the source distribution, namely,

$$
\sigma=-\bar{U} \cdot \hat{n}
$$

Uniqueness of solutions with $\phi=0$ and $\sigma=-\overline{\mathrm{U}} \cdot \hat{\mathrm{n}}$ boundary conditions depends only upon uniqueness for solutions of the integral equation (3.48) for $\mu$. Discretization of equation (3.48) by a panel method leads to a set of simultaneous equations of the form

$$
\mathrm{a}_{\mathrm{ij}} \mu_{\mathrm{j}}=\mathrm{b}_{\mathrm{i}} \quad \mathrm{i}, \mathrm{j}=1,2,3, \ldots, \mathrm{n}(\mathrm{j} \text { summed })
$$

where $\mathrm{i}, \mathrm{j}$ denotes the points at which $\mathrm{b}_{\mathrm{i}}$ is evaluated and $\mu_{\mathrm{j}}$ are values of the doublet strength to be delermined. Because of the nature of the kernel function

$$
\frac{\mathrm{B}^{2}\left(\overline{\mathrm{R}}_{0}-\overline{\mathrm{R}}\right) \cdot \hat{\mathrm{n}}}{\mathrm{R}_{\mathrm{B}}{ }^{3}}
$$


the $\mathrm{a}_{\mathrm{ij}}$ are diagonally dominant. When the matrix is of rank $\mathrm{n}$ then the solution of the discrete values of $\mu$ are unique. When the discretization is properly carried out, then we have shown that the solution with the potential boundary conditions

$$
\sigma=-\overline{\mathrm{U}} \cdot \hat{\mathrm{n}} \quad \phi^{-}=0
$$

is unique and satisfies the condition that the normal component of linear mass flux vanish on the exterior $(+)$ surface.

We shall now consider uniqueness of linearized supersonic flow solutions when linearized boundary conditions are applied on a plane $z=0$. The quantity $\bar{U} \cdot \hat{n}$ is then identically zero on the surface and the surface integral in equation (3.45) becomes

$$
\begin{aligned}
& \left(U_{0} / 2\right) \int_{S_{3}}\left(B^{2} u^{2}+v^{2}+w^{2}\right) d y d z \\
& +\int_{S_{w}} \bar{U} \cdot \bar{V}^{+} \bar{W}^{+} \cdot \hat{n} d s \\
& +\int_{S_{w}} \bar{U} \cdot \bar{V}^{-} \bar{W}^{-} \cdot \hat{n} d s=0
\end{aligned}
$$

In the same manner we let $\bar{V}$ and $\bar{W}$ represent the difference between two solutions with the same boundary conditions, then we see that the solution is unique if either

$$
\overline{\mathrm{U}} \cdot \overline{\mathrm{V}}
$$

or

$$
\overline{\mathrm{W}} \cdot \hat{\mathrm{n}}
$$

is prescribed over the surface $S_{W}$. The quantity $\bar{U} \cdot \bar{V}$ is the linearized pressure and, hence, is the design type houndary condition while $\bar{W} \cdot \hat{n}=\partial \phi / \partial z$ is the down wash boundary condition relating to the wing slope. From the form of the integral over $\mathrm{S}_{\mathrm{W}}$, solutions in which part of the surface has design type boundary conditions and part downwash boundary conditions are also unique. 


\subsection{THE VELOCITY COMPONENTS NEGLECTING VORTICES AT PANEL EDGES}

The velocity components can be computed by differentiating equation (3.39). This approach will lead to integrals containing the doublet strength which will introduce vortices at panel edges when doublet strength is not continuous. Another form of the velocity vector which contains no integrals of the doublet strength to produce these vortices is obtained by using the identity established by Ward in appendix 1 of reference [10], i.e.,

$$
\begin{aligned}
\int_{S}\left(\bar{v}_{1} \bar{W}_{2} \cdot \hat{n}+\bar{V}_{2} \bar{W}_{1} \cdot \hat{n}-\bar{V}_{1} \cdot \bar{W}_{2} \hat{n}\right) d s= \\
\int_{V}\left[\bar{V}_{1} \nabla \cdot \bar{W}_{2}+\bar{V}_{2} \nabla \cdot \bar{W}_{1}-\bar{W}_{1} \times\left(\nabla \times \bar{V}_{2}\right)-\bar{W}_{2} \times\left(\nabla \times \bar{V}_{1}\right)\right] d v
\end{aligned}
$$

If the volume is source free and the flow is irrotational, the volume integral vanishes. Let $W_{2}$ and $V_{2}$ be given by the source in equation (3.28) and the surface $S$ contain a surface $\mathrm{S}_{\mathrm{W}}$ on which boundary conditions are to be applied, a characteristic surface from the leading edge of the wing surface $S_{\mathrm{W}}$, the cone from the point $\mathrm{x}, \mathrm{y}, \mathrm{z}$ and a circular cross section $S_{1}$ cutting the Mach cone close to the singularity at $x_{0}=x, y_{0}=y$, and $z_{0}=z$, as shown in figure 10 . Since $\mathrm{V}_{2} \mathrm{~W}_{1}=\mathrm{V}_{1} \mathrm{~W}_{2}$, the last two terms of the integrand of equation (3.49) become

$$
\bar{V}_{2} \bar{w}_{1} \cdot \hat{n}-\bar{V}_{1} \cdot \bar{w}_{2} \hat{n}=\bar{V}_{2} \bar{w}_{1} \cdot \hat{n}-\bar{V}_{2} \cdot \bar{w}_{1} \hat{n}=\bar{w}_{1} \times\left(\bar{v}_{2} \times \hat{n}\right)
$$

Now, as the distance from the singularity of $S_{1}$ at $x, y, z$ goes to zero, we have

$$
\int_{S_{1}}^{*}\left[\bar{V}_{1} \overline{\mathrm{W}}_{2} \cdot \hat{\mathrm{n}}+\overline{\mathrm{W}}_{1} \times\left(\overline{\mathrm{V}}_{2} \times \hat{\mathrm{n}}\right)\right] \mathrm{ds} \simeq \overline{\mathrm{V}}_{1} \int_{\mathrm{S}_{1}}^{*} \overline{\mathrm{W}}_{2} \cdot \hat{\mathrm{n}} \mathrm{ds}+\overline{\mathrm{W}}_{1} \times \int_{\mathrm{S}_{1}}^{*}\left(\overline{\mathrm{V}}_{2} \times \hat{\mathrm{n}}\right) \mathrm{ds}
$$

The first integral has been evaluated for the integral equation of $\phi$ and Ward [10] has shown that the second integral is zero, hence,

$$
\int_{S}^{*}\left[\overline{\mathrm{V}}_{1} \overline{\mathrm{W}}_{2} \cdot \hat{\mathrm{n}}+\overline{\mathrm{W}}_{1} \times\left(\overline{\mathrm{V}}_{2} \times \hat{\mathrm{n}}\right)\right] \mathrm{ds}=2 \pi \overline{\mathrm{V}}_{1}(\overline{\mathrm{R}})
$$

After substituting $\bar{V}_{1} \bar{W}_{2} \cdot \hat{n}-\bar{V}_{1} \cdot \bar{W}_{2} \hat{n}=\left(\hat{n} \times \bar{V}_{1}\right) \times \bar{W}_{2}$, the relation for the velocity vector becomes, after dropping the subscript 1 ,

$$
\bar{V}(\bar{R})=-\frac{1}{2 \pi} \int_{S_{W}}^{*}\left[\bar{V}_{2}(\hat{n} \cdot \bar{W})+(\hat{n} \times \bar{V}) \times \bar{W}_{2}\right] d s
$$


Substituting for $\overline{\mathrm{V}}_{2}$ and $\overline{\mathrm{W}}_{2}$ from equation (3.28) yields

$$
\bar{V}(\bar{R})=-\frac{1}{2 \pi} \int_{S_{W}}^{*} \hat{n} \cdot \bar{W}\left(\bar{R}_{0}\right) \nabla\left(\frac{1}{R_{B}}\right) d s-\frac{B^{2}}{2 \pi} \int_{S_{W}}^{*} \frac{\left[\hat{n} \times \bar{V}\left(\bar{R}_{0}\right)\right] \times\left(\bar{R}_{0}-\bar{R}\right) d s}{R_{B}}
$$

If the panel is superinclined, $\hat{n} \times \bar{V}\left(R_{0}\right)$ and $\bar{W}\left(R_{0}\right) \cdot \hat{n}$ take on their appropriate values on the surface $S_{W}$. For the subinclined surface we incorporate the region on the opposite side of the surface lying within the Mach cone from the point $\mathrm{x}, \mathrm{y}, \mathrm{z}$ as we did for the velocity potential. This makes no contribution to $V(R)$ and we obtain

$$
V(R)=-\frac{1}{2 \pi} \int_{S_{W}} \sigma\left(R_{0}\right) \nabla\left(\frac{1}{R_{B}}\right) d s \quad-\frac{B^{2}}{2 \pi} \int_{S_{W}}^{*} \frac{(\hat{n} \times \nabla \mu) \times\left(\overline{R_{0}}-\bar{R}\right) d s}{R_{B}{ }^{3}}
$$

where $\sigma=\bar{W}^{+} . \hat{n}-\overline{\mathrm{W}} \cdot \hat{\mathrm{n}}$ is the source distribution on the surface and $\mu=\phi^{+}-\phi^{-}$is the doublet distribution. The quantity $\hat{n} \times \nabla \mu$ is the vorticity vector lying on the surface $S_{W}$. Notc that the doublet integral contains only derivatives of $\mu$. This is the form for the velocity applied to panels when line integrals of the doublet distribution are to be excluded. The integral may also be written

$$
\begin{aligned}
-\frac{\mathrm{B}}{2 \pi} \int_{\mathrm{S}_{\mathrm{W}}}^{*} \frac{(\hat{\mathrm{n} \times \nabla \mu}) \cdot\left(\overline{\mathrm{R}}_{0}-\overline{\mathrm{R}}\right) \mathrm{ds}}{\mathrm{R}_{\mathrm{B}}^{3}}= & -\frac{\mathrm{B}^{2}}{2 \pi} \int_{\mathrm{S}_{\mathrm{W}}}^{*} \frac{\left.\nabla \mu \overline{\mathrm{R}}_{0}-\overline{\mathrm{R}}\right) \cdot \hat{\mathrm{n}}}{\mathrm{R}_{\mathrm{B}}{ }^{3} \mathrm{ds}} \\
& +\frac{\mathrm{B}^{2}}{2 \pi} \int_{\mathrm{S}_{\mathrm{W}}}^{*} \frac{\left(\overline{\mathrm{R}}_{0}-\overline{\mathrm{R}}\right) \cdot \nabla \mu \hat{\mathrm{n} d s}}{\mathrm{R}_{\mathrm{B}}{ }^{3}}
\end{aligned}
$$

\subsection{BOUNDARY CONDITIONS ON WINGS AND BODIES}

We have shown that unique solutions are obtained by prescribing the potential and the linear mass flux boundary conditions on the downstream side of surfaces inclined at angles to the free stream greater than the Mach angle, i.e., superinclined surfaces. When the flow is subsonic, the solution can be shown to be unique if either the potential or the normal linear mass flux is prescribed on any surface. There is no similar proof for subinclined surfaces in supersonic flow. When linearized boundary conditions are prescribed on a plane then the solution is unique when either the linearized mass flux or the linear 
pressure are prescribed. Furthermore, the solution is also unique if mass flux boundary conditions are prescribed over part of the surface and linear pressure boundary conditions are prescribed over the remainder.

Using either a source or a doublet distribution alone on a closed surface such as a wing or body, yields an interior flow with disturbances propagated along Mach lines and reflected from the surface and causing unrealistic variations in the exterior pressure distribution. By utilizing a combined source and doublet distribution on each panel, it is possible to eliminate the interior flow for a wing or body and to provide a smoother pressure distribution on the exterior of the wing or body. We have shown in section 3.8 that choosing the source strength $\sigma$ according to

$$
\sigma=-\overline{\mathrm{U}} \cdot \hat{\mathrm{n}}
$$

and setting

$$
\phi=0
$$

on the interior of the surface, produces the appropriate boundary conditions of no mass flux through the surface,

$$
\bar{W} \cdot \hat{n}=-\bar{U} \cdot \hat{n}
$$

To preserve continuity of doublet strength, vortex sheets are shed from the trailing edges of wings. When the trailing edge is supersonic, the flow at any point on the edge is not influenced by any other point on the edge. The flow then changes abruptly across the trailing edge as in two-dimensional flow. On subsonic edges, the flow at any point on the edge is influenced by all points along the edge contained in the Mach cone upstream of that point. The flow must leave the wing smoothly and a Kutta condition is applied to all such edges. Special planar wake panels preserve continuity of the doublet strength with the wing trailing edges. The doublet strength on the panels depends only upon the variable normal to the flow dircction. Thus, the lincarized pressure jump

$$
\Delta \mathrm{C}_{\mathrm{p}}=-2 \partial \mu / \partial \mathrm{x}=0
$$

is a necessary requirement since the doublet sheet representing a shed wake must not support a pressure differential. 


\subsection{GENERAL DESCRIPTION OF THE SUPERSONIC PANEL METHOD}

\subsection{EARLIER SIX PARAMETER SPLINE METHOD}

It was shown in section 3 that solutions to the linearized supersonic flow over a configuration can be expressed in terms of distributions of source and doublet strength on the configuration surface. Except for the simplest of geometries, complete analytic determination of the source and doublet strength from the integral equation defining the boundary conditions is impractical. To overcome this difficulty, the panel methods were devised.

The panel method is basically a collocation method for satisfying the boundary conditions at a discrete set of points in order to evaluate a corresponding set of source and doublet strengths. The source and doublet distributions on the panel are defined in terms of the unknown values of the singularities at the centers of the panel and of neighboring panels by a system of spline type polynomials. The resulting integrals over the panels representing the velocity components and potential at the control points on the panels can be integrated in closed form, and imposing boundary conditions yields a set of simultaneous equations to be solved for the singularity parameters. When these quantities are known, the flow field velocity and pressure can readily be computed.

An example of a paneled wing and body combination is shown in figure 11 . A sufficiently fine set of grid points is defined over the surface. By joining the grid points with straight lines, we form the basic set of quadrilateral panels, which in some special cases reduce to triangles. Generally, these quadrilaterals are not planar. One can easily show that the midpoints of the four sides of the quadrilateral lie on a plane which we shail call the average plane of the panel. In figure 12 the vector representing the side $\mathrm{P}_{6}-\mathrm{P}_{5}$ of the inner quadrilateral is given by

$$
\overline{\mathrm{P}}_{6}-\overline{\mathrm{P}}_{5}=\left(\overline{\mathrm{P}}_{1}+\overline{\mathrm{P}}_{2}\right) / 2-\left(\overline{\mathrm{P}}_{1}+\overline{\mathrm{P}}_{4}\right) / 2=\left(\overline{\mathrm{P}}_{2}-\overline{\mathrm{P}}_{4}\right) / 2
$$

Similarly, the vector of the opposite side $\mathrm{P}_{7}-\mathrm{P}_{8}$ is

$$
\overline{\mathrm{P}}_{7}-\overline{\mathrm{P}}_{8}=\left(\overline{\mathrm{P}}_{2}+\overline{\mathrm{P}}_{3}\right) / 2-\left(\overline{\mathrm{P}}_{4}+\overline{\mathrm{P}}_{3}\right) / 2=\left(\overline{\mathrm{P}}_{2}-\overline{\mathrm{P}}_{4}\right) / 2=\overline{\mathrm{P}}_{6}-\overline{\mathrm{P}}_{5}
$$

We see that the sides are parallel and $\mathrm{P}_{5} \mathrm{P}_{6} \mathrm{P}_{7} \mathrm{P}_{8}$ forms a parallelogram. Following the subsonic mcthod of Johnson and Rubbert [3], the earlier supersonic program utilized quadratic doublet and linear source distributions prescribed over the projection of the panel onto the average plane. Thus, the panels inducing the flow did not form a continuous closed surface for non-planar configurations, but contained gaps between the panel edges.

Essentially, two boundary conditions are required at the center point of each panel and associated with each point are the values of the source and doublet strength to be determined. The six coefficients of the quadratic for the doublet distributions and the three coefficients for the linear source distribution on each panel, are determined by least 
square fit with the unknown values of the doublet and source strengths at the centers of the panel and of neighboring panels. A system of splines then defines the doublet and source strength distributions over the surface of the configuration, although, they do not have the continuity usually associated with conventional splines.

Because the spline fit requires surfaces with a certain degree of smoothness, complex configurations such as wings and bodies are divided up into networks. At junctions of networks, discontinuities in curvature and slope are allowed. To match doublet strength across network edges, additional control points at panel edges are provided. Mathematically, a network is a rectangular array of panels over which a set of control points, with appropriate boundary conditions, and source and doublet parameters are defined to provide a determinate system. Figures 13 and 14 schematically show control point and singularity locations for the source and for the doublet networks with analysis boundary conditions, respectively. Figure 15 shows the location of the control points and the singularity values for the doublet design network used in the single planar example described in section 12 . When the flow is required over a known body shape, source and doublet strengths defined at these same points determine the source and doublet distribution over the entire network by the spline system.

The three parameters to define the source strength and the six parameters to define the doublet distributions are not sufficient to ensure complete continuity of the source and doublet distribution at panel edges. As shown in section 6, discontinuity in the doublet strength induces higher order singularities in the flow than a similar discontinuity in source strength. The six parameter doublet distribution can be made to agree at panel corner points only or can be made continuous only in a least square sense across panel edges. Some excellent results using this spline were obtained and are discussed in section 12 . However, for some paneling of certain configurations, the effects of the panel edge gaps and slight discontinuity of doublet strength were found to build up and lead to instability in the solution. To distinguish this earlier spline system from the spline system to be described in the following, we shall call it the six parameter spline.

Ideally, the doublet strength at network edges should be as strictly continuous as it is at panel and subpancl edges. However, the doublet strength can be made almost continuous for the six parameter spline by moving the control points an infinitesimal distance away from the boundaries into the panel and using the infinite singularities of the aerodynamic influence coefficients as shown in section 6 . This approach fails for supersonic leading edges where only finite discontinuities occur. To match the doublet strength at supersonic network edges, incompressible aerodynamic influence coefficients are used for all network edges. The inconsistency is only apparent because the role of the aerodynamic influence coefficient is to make the doublet strength and its conormal derivative nearly continuous. For greater details on the six parameter spline, the reader is referred to Johnson and Rubbert [3]. 


\subsection{IMPROVED NINE PARAMETER DOUBLET SPLINE AND IMPROVED PANELING TO OBTAIN CONTINUOUS DOUBLET STRENGTH AND GEOMETRY}

To establish a panel system which is a completely closed polyhedral surface, we divide each quadrilateral panel into 8 triangles. We have seen that the 4 midpoints of the sides of the panel lie in a plane, and the lines joining these midpoints actually form a parallelogram (see fig. 12). Each corner point, together with the midpoints of the lines meeting at that corner, form a triangle which has a common edge with the parallelogram. Thus, the conventional quadrilateral panel can be divided into 5 planar surfaces whose edges are contiguous and which are contiguous with the edges of adjoining panels. When the center parallelogram is divided into 4 triangles, the doublet can be made continuous across all panels and subpanel edges by using the nine points in figure 12 to define a separate quadratic distribution in each subpanel. Because of this feature, we shall call this splinal system the nine parameter spline. Since the center of the panel where values of the source and doublet strength are defined is the common vertex of the four panels, the control point at which the boundary conditions are applied must be displaced from the center to avoid numerical difficulties with the panel influence coefficients which are indeterminate at panel corner points where $s=s_{m}=z=R=0$ (see, for example, equations (A15) and (A16)). Since the nine parameter spline renders the doublet strength continuous and there are no gaps in the edges, the acrodynamic influcnec coefficients can be simplified by eliminating all line integrals of the doublet strength around panel edges as these integrals will cancel in the summing of the aerodynamic influence coefficients from the adjacent panels. For greater details in the construction of the spline, refer to appendix C.

The nine parameter spline insures continuity of doublet strength everywhere within a network. In the latest version of the program, the doublet is made strictly continuous also across network edges, by appropriate boundary conditions at the control points on the network edges (see fig. 14). Consider two networks, the second lying downstream of the first. On the downstream edge of the first network, regular downwash boundary conditons are applied to determine the values of the doublet strength. These values of the doublet strength are then used to define the doublet strength at these control points common with the adjoining downstream network. By means of the spline system, the doublet strengths are defined at the panel corners along the network edge in terms of the values at the network edge control points, and are restricted to have the same values for both networks. Since there are three points on each panel at which the doublet strength is defined along the network edge and a cubic spline is used, the doublet strength is then made continuous on all points of the common boundary between the two networks.

Since some of the example flows discussed in section 12 were calculated using less strict matching of the doublet strength, it is worthwhile, therefore, to discuss this indirect method of nearly matching the doublet strength across network boundaries. In section 6 , an explanation of how the singularity in velocity from the line integral of doublet strength across network edges is presented. Since these line integrals have been eliminated from the aerodynamic influence coefficients used in the 9 parameter spline, a simulated incompressible vortex was added to the conventional mass flux boundary conditions. To achieve a match of the doublet strength across network edges, the boundary points were moved an infinitesimal distance into the network and away from the panel boundary where the influence coefficients 
become infinite like $\gamma / \mathrm{r}$ where $\mathrm{r}$ is the distance from the edge and $\gamma=\Delta \mu$ is the vorticity magnitude. Thus, at two points on opposite sides of the network boundary we have

$$
\begin{aligned}
& \left(\overline{\mathrm{W}}_{1}+\overline{\mathrm{U}}\right) \cdot \hat{\mathrm{n}}_{1}+\left(\mu_{1}-\mu_{2}\right) / \mathrm{r}_{1}=0 \\
& \left(\overline{\mathrm{W}}_{2}+\overline{\mathrm{U}}\right) \cdot \hat{\mathrm{n}}_{2}+\left(\mu_{2}-\mu_{1}\right) / \mathrm{r}_{2}=0
\end{aligned}
$$

where $r_{1}$ and $r_{2}$ are distances from the points 1 and 2 , respectively, to the network edges. These equations may be combined to give

$$
\mu_{1}-\mu_{2}=\left(\bar{W}_{1}+\bar{U}\right) \cdot \hat{n}_{1} r_{1}-\left(\bar{W}_{2}+\bar{U}\right) \cdot \hat{n}_{2} r_{2} \approx 0
$$

and

$$
r_{1}\left(\bar{W}_{1}+\bar{U}\right) \cdot \hat{n}+r_{2}\left(\bar{W}_{2}+\bar{U}\right) \cdot \hat{n}_{2}=0
$$

Hence, the doublet strength is nearly matched and mass flux boundary conditions are satisfied in a weighted average.

\subsection{COMBINED SOURCE $A$ ND DOUBLET PANELS}

On aircraft bodies and wings without lift, it may be possible to find the linearized solutions with sources alone on the surface. For such closed configurations there results both exterior and interior flows. When the flow is supersonic the interior flow may cause build-up of disturbances which propagate along Mach lines and are reflected from the surface in the downstream direction as shown in figure 16. These waves may produce large pressure disturbances on the exterior of the surface. Such interference waves can be eliminated by combined source and doublet paneling on the configuration.

In section 3.8 we showed that when the source strength at the control points is set equal to $-\bar{U} \cdot \hat{n}$, i.e.

$$
\sigma=-\overline{\mathrm{U}} \cdot \hat{\mathrm{n}}
$$

and the boundary conditions

$$
\phi=0
$$

are prescribed at points on the inner boundary, the boundary conditions

$$
\bar{W} \cdot \hat{n}=-\bar{U} \cdot \hat{n}
$$

result on the outside surface. Furthermore, the interior perturbation flow is zero, therefore eliminating the interior waves and the resulting disturbances on the exterior surface. 
The application of boundary conditions in equations (4.3) and (4.4) on the surface of a flow-through nacclle will only work on an isolated naccllc in supersonic flow where there is no incident perturbation flow, for which $\phi$ is automatically zero on the inlet surface. When a nacelle is situated in the region of influence of a wing, the inlet can be closed by means of superinclined panels, which absorb or cancel the incident flow. Boundary conditions can only be applied to the downstream side of the superinclined surface; and to eliminate the incident perturbation flow, these boundary conditions, as explained in section 3.6 , are

$$
\phi=\overline{\mathrm{W}} \cdot \hat{\mathrm{n}}=0
$$

It is also possible to represent a jet exhaust by terminating a nacelle with a superinclined network. For this case the normal mass flux would be prescribed, namely,

$$
\bar{W} \cdot \hat{n}=f(\xi, \hat{n})
$$

The jet exhaust cannot be represented exactly, since the issuing jet has the same total head as the free stream flow because it satisfies the same differential equation. A convenient second boundary condition on the exit surface is $\phi=0$; and to match doublet strength a trailing doublet wake must be included. The shape of the sheet is not known but it may be approximated by a cylinder.

Thin wings can also be represented by sources and doublets distributed over the planar planform. The thickness distribution is represented by sources, while camber shape (or angle of attack) is defined by doublets. Examples of cambered wings and flat plates at angles of attack and of symmetrical thick wings are presented in section 12 along with comparison with exact linearized solutions.

\subsection{EFFICIENT COMPUTATION OF THE INFLUENCE COEFFICIENTS}

The influence coefficients were derived in such a way that they give valid results when all or part of the panel edge lies outside the zone of influence of the control point. However, since their computation is fairly costly, considerable economy in computation is achieved by using a subroutine to test whether a panel is in the zone of the given control point and eliminating computation of the influence coefficients for those panels lying outside. The first test is to find out if any corner of the panel is within the upstream Mach conc from the control point by computing the square of the hyperbolic distance from each corner. If it is positive for any corner, then computation of the influence coefficients is carried out. If it is negative for all corners, then a further test is made to see whether any edges cuts the Mach cone. If so, then the computation of the panel influence coefficients is carried out. If not, then the program proceeds to the next panel without entering the influence coefficient subroutine, unless the panel happens to be in a superinclined network. For superinclined panels, a further test is required to find whether the intersection of the Mach cone with the panel lies entirely inside the panel. 
A further economy in computation is achieved by recognizing that some terms of the influence coefficients are linear combinations of a smaller set. Also, since the terms such as equations (A15) and (A16) are evaluated at the end points of the edges, the inverse tangents and logarithms from the two endpoints of each cdge can bc combined, requiring one half the necessary entries into the arc tangent and logarithm subroutines, which are costly in computing time.

For panels far away from the control point and lying well within the Mach cone, far field expansions of the aerodynamic influence coefficients may be used. Since these are algebraic in form they require less computing time than the near field aerodynamic influence coefficients. 


\subsection{AERODYNAMIC INFLUENCE COEFFICIENTS FOR SUBINCLINED DOUBLET PANELS}

To represent the aircraft configuration with sufficient fidelity by means of the simple panels described in the foregoing section, it must be divided into panels sufficiently small that the curvature of the panel is small in relation to the panel's smallest dimension. The panel shape can then be represented by a simple second degree polynomial. Linear distributions of source strength and quadratic distribution of doublet strength are utilized enabling the contribution to the velocity potential from each panel to be integrated in closed form.

To obtain the simplest form for the influence coefficients, we scale the variables according to

$$
(\mathrm{x}, \mathrm{y}, \mathrm{z})=\left(\mathrm{x}_{\mathrm{C}}, \mathrm{By}_{\mathrm{C}}, \mathrm{Bz}_{\mathrm{c}}\right)
$$

where $x_{c}, y_{C}, z_{c}$ designates the compressible coordinate system, so named because $x_{c}$ is in the direction of the free stream velocity. This transformation eliminates the factor $\mathrm{B}$ from the hyperbolic distance $R_{B}$. For the six parameter spline, we represent the subinclined panel as a small quadratic departure from the plane $z=0$ and integration is performed over the projection on the plane $z=0$. For the nine parameter spline, the subpanels themselves are planar and are assumed to lie in the plane $z=0$. Choosing the subinclined panel as the $z=0$ plane also simplifies the conormal derivative to $\partial / \partial z$, further simplifying the in tegral for the doublet. For subpanels or panels not lying in the $z=0$ plane, the influence coefficients are transformed by coordinate transformations which are discussed in section 10 .

The integration over the panel may be accomplished by combining the integrations of regions bounded by a panel edge, two lines of constant $y$ and the intersection of the Mach cone on the plane $z=0$ as shown in figure 17. This can be seen by considering figure 18 for a panel which lies wholly within the Mach cone. The integration over the area between lines $A B$ and $B C$ are added together. Since $C D$ and $D A$ are taken in the reverse order, the integration over the area between these two lines are subtracted from the areas under $A B$ and $\mathrm{BC}$. The area remainding is the desired area of the quadrilateral $\mathrm{ABCD}$ over which the integration is required. The integration over the region in figure 17 , yields a quantity to be evaluated at the endpoints of the panel edge. The induced flow from the panel is then computed by evaluating the function at the endpoints of each side proceeding around the panel in a counter-clockwise direction.

Let the panel surface be defined by

$$
F\left(x_{0}, y_{0}, z_{0}\right)=z_{0}-\zeta\left(x_{0}, y_{0}\right)=0
$$

Then to the first order in $\zeta$, the normal to the surface becomes

$$
\mathrm{n}_{\mathrm{z}_{0}}=1+0\left(\zeta^{2}\right), \mathrm{n}_{\mathrm{x}_{0}}=-\zeta_{\mathrm{x}_{0}}+0\left(\zeta^{2}\right), \mathrm{n}_{\mathrm{y}_{0}}=-\zeta_{\mathrm{y}_{0}}+0\left(\zeta^{2}\right)
$$

where $\zeta_{\mathrm{x}_{0}}=\partial \zeta / \partial \mathrm{x}_{0}$ and $\zeta_{\mathrm{y}_{0}}=\partial \zeta / \partial_{\mathrm{y}_{0}}$ 
We shall consider the doublet term first. Thus, with $\psi=1 / R_{\mathrm{S}}$ where $R_{S}=\sqrt{\left(x-x_{0}\right)^{2}-\left(y-y_{0}\right)^{2}-\left(z-z_{0}\right)^{2}}$, equation (3.43) becomes

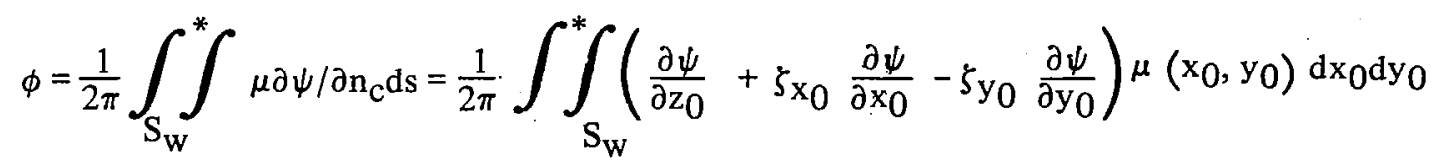

where $\mathrm{ds}=\mathrm{dx}_{0} \mathrm{dy}_{0} / \cos \Omega=\mathrm{dx}_{0} \mathrm{dy}_{0}+0\left(\xi^{2}\right), \partial / \partial \mathrm{n}_{\mathrm{c}}=\left(-\mathrm{n}_{\mathrm{x}}, \mathrm{n}_{\mathrm{y}}, \mathrm{n}_{\mathrm{z}}\right) \cdot \nabla, \hat{\mathrm{n}}=\left(\mathrm{n}_{\mathrm{x}}, \mathrm{n}_{\mathrm{y}}, \mathrm{n}_{\mathrm{z}}\right)$ is the normal of the wing or body surface, and $\Omega$ is the angle of projection of the elem ent of the area on the average plane. The sign of equation (3.43) has been changed to conform to the general practice of defining the body and wing normals outward into the fluid, while the development of the general theory in section 3 is based on an outward normal from the fluid region.

Since the finite part contains no terms from differentiation of the limits of integration, we may move the differentiation outside of the integral sign by noting that

$$
\frac{\partial \psi}{\partial \mathrm{x}_{0}}=-\frac{\partial \psi}{\partial \mathrm{x}^{3}} \frac{\partial \psi}{\partial \mathrm{y}_{0}}=-\frac{\partial \psi}{\partial \mathrm{y}}, \frac{\partial \psi}{\partial \mathrm{z}_{0}}=-\frac{\partial \psi}{\partial \mathrm{z}}
$$

Therefore, for the velocity potential we obtain

$$
\begin{aligned}
\phi= & -\frac{1}{2 \pi} \frac{\partial}{\partial z} \int_{\mathrm{y}_{1}}^{\mathrm{y}_{2}} \int_{\xi\left(\mathrm{y}_{0}\right)}^{\ell\left(\mathrm{y}_{0}\right)} \frac{\mu\left(\mathrm{x}_{0}, \mathrm{y}_{0}\right) \mathrm{dx_{0 } \mathrm { d } \mathrm { y } _ { 0 }}}{\mathrm{R}_{\mathrm{s}}} \\
& -\frac{1}{2 \pi} \frac{\partial}{\partial \mathrm{x}} \int_{\mathrm{y}_{1}}^{\mathrm{y}_{2}} \int_{\xi\left(\mathrm{y}_{0}\right)}^{\ell\left(\mathrm{y}_{0}\right)} \frac{\mu\left(\mathrm{x}_{0}, \mathrm{y}_{0}\right) \zeta_{\mathrm{x}_{0}} \mathrm{dx}_{0} \mathrm{dy}_{0}}{\mathrm{R}_{\mathrm{s}}} \\
& +\frac{1}{2 \pi} \frac{\partial}{\partial \mathrm{y}} \int_{\mathrm{y}_{1}}^{\mathrm{y}_{2}} \int_{\xi\left(\mathrm{y}_{0}\right)}^{\ell\left(\mathrm{y}_{0}\right)} \frac{\mu\left(\mathrm{x}_{0}, \mathrm{y}_{0}\right) \zeta_{\mathrm{y}_{0}} \mathrm{dx_{0 } \mathrm { dy } _ { 0 }}}{\mathrm{R}_{\mathrm{s}}}
\end{aligned}
$$

with

$$
R_{s}=\sqrt{\left(x-x_{0}\right)^{2}-\left(y-y_{0}\right)^{2}-(z-\zeta)^{2}}
$$

The finite part symbol is removed when integration is performed before differentiation since the integral is then made convergent. Here

$$
\zeta=\zeta\left(\mathrm{x}_{0}, \mathrm{y}_{0}\right) \text { and } \mathrm{x}_{0}=\xi\left(\mathrm{y}_{0}\right)
$$


is the equation of the projection on the plane $\mathrm{z}=0$ of the intersection of the Mach cone with the panel surface. The region of integration is shown in figure 17 . We simplify the Mach cone singularity by introducing a new variable for $\mathrm{x}_{0}$. Let

$$
\mathrm{x}_{0}=\xi\left(\mathrm{y}_{0}\right)-\mathrm{t}=\xi_{0}-\mathrm{t}
$$

and assume a straight edge

$$
\mathrm{x}_{0}=\mathrm{x}_{1}+\left(\mathrm{y}_{0}-\mathrm{y}_{1}\right) / \mathrm{m}
$$

where $\mathrm{m}=\left(\mathrm{y}_{2}-\mathrm{y}_{1}\right) /\left(\mathrm{x}_{2}-\mathrm{x}_{1}\right)$ is the slope of the panel edge. By this transformation $t=0$ represents the Mach cone boundary. The hyperbolic distance becomes

$$
R_{s}=\sqrt{\left(x-\xi_{0}+t\right)^{2}-\left(y-y_{0}\right)^{2}-\left[z-\zeta\left(\xi_{0}-t, y_{0}\right)\right]^{2}}
$$

Expanding in the form

$$
\zeta\left(\xi_{0}-t, y_{0}\right)=\zeta\left(\xi_{0}, y_{0}\right)-t \xi_{x}\left(\xi_{0}, y_{0}\right)+t^{2} \zeta_{x x} / 2
$$

and using the condition that

$$
\sqrt{\left(x-\xi_{0}\right)^{2}-\left(y-y_{0}\right)^{2}-\left[z-\zeta\left(\xi_{0}, y_{0}\right)\right]^{2}}=0
$$

yields for the hyperbolic distance

$$
\left.\sqrt{t\left[a_{1} t+b_{1}+o\left(\zeta^{2}\right)\right.}\right]
$$

where

$$
\begin{aligned}
& \mathrm{a}_{1}=1+\left(\mathrm{z}-\zeta_{0}\right) \zeta_{\mathrm{xx}} \simeq 1+\mathrm{z} \zeta_{\mathrm{xx}}+0\left(\zeta^{2}\right) \\
& \mathrm{b}_{1}=2\left[\left(\mathrm{x}-\xi_{0}\right)-\mathrm{z} \zeta_{0 \mathrm{x}}\right] \\
& \zeta_{0}=\zeta\left(\xi_{0}, \mathrm{y}_{0}\right)
\end{aligned}
$$


To find the equation for the Mach line boundary we consider

$$
\begin{gathered}
\left(x-\xi_{0}\right)=\sqrt{\left(y-y_{0}\right)^{2}+\left(z-\xi_{0}\right)^{2}}=r-z \xi_{0} / r+0\left(\xi^{2}\right) \\
\text { or } \xi_{0}=x-r+z \xi_{0} / r+0\left(\xi^{2}\right)
\end{gathered}
$$

where $r=\sqrt{\left(y-y_{0}\right)^{2}+z^{2}}$. Then $\zeta\left(\xi_{0}, y_{0}\right) \simeq \zeta\left(x-r, y_{0}\right)+0\left(\zeta^{2}\right)$

Finally retaining only first order terms in $\zeta$ yields for the potential

$$
\begin{aligned}
\phi=\frac{1}{2 \pi} & \left\{\frac{\partial}{\partial z} \int_{y_{1}}^{y_{2}} \int_{0}^{x-x-r-\left(y_{0}-y\right) / m+\epsilon_{1}} \frac{\mu\left(x-r-t+\epsilon_{1}, y_{0}\right) d t d y_{0}}{\sqrt{t\left(a_{1} t+b_{1}\right)}}\right. \\
& +\frac{\partial}{\partial x} \int_{y_{1}}^{y_{2}} \int_{0}^{x-x_{1}-r-\left(y_{0}-y_{1}\right) / m} \frac{\zeta_{x}\left(x-r-t, y_{0}\right) \mu\left(x-r-t, y_{0}\right) d t d y_{0}}{\sqrt{t(t+2 r)}} \\
& \left.-\frac{\partial}{\partial y} \int_{y_{1}}^{y_{2}} \int_{0}^{x-x_{1}-r-\left(y_{0}-y_{1}\right) / m} \frac{\zeta_{y}\left(x-r-t, y_{0} \mu\left(x-r-t, y_{0}\right) d t d y_{0}\right.}{\sqrt{t(t+2 r)}}\right\}
\end{aligned}
$$

where

$$
\begin{array}{ll}
\epsilon_{1}=\mathrm{z} \zeta / \mathrm{r} & \epsilon_{2}=\mathrm{z} \zeta_{\mathrm{xx}}
\end{array} \quad \epsilon_{3}=\mathrm{z}\left(\zeta_{\mathrm{x}}+\zeta / \mathrm{r}\right)
$$

The integration of equation 5.10 is carried out in appendix B retaining only the first order terms in the epsilons and $\zeta$. It was found that the contributions to the velocity by the first order terms have higher order infinite singularities near the Mach cones emanating from panel corners and hence, are not a true correction for panel curvature. Consequently, the influence coefficients for the flat panels only are used. Setting $\zeta=0$ in equation 5.10 yields

$$
\phi=\frac{1}{2 \pi} \frac{\partial}{\partial z} \int_{y_{1}}^{y_{2}} \int_{0}^{x-x_{1}-r-\left(y_{0}-y_{1}\right) / m} \frac{\mu\left(x-r-t, y_{0}\right) d t d y_{0}}{\sqrt{t(2 r+t)}}
$$


The integral may be simplified by introducing the following variables:

$$
\begin{aligned}
& x_{m}=x-x_{1}-\left(y-y_{1}\right) / m, s=y-y_{0} \\
& s_{m}=x_{m}+s / m
\end{aligned}
$$

Scaling the $t$ variable by $r$ eliminates differentiation of the singular part of the integrand allowing differentiation to be performed before integration. We obtain for the perturbation potential

$$
\phi=-\frac{1}{2 \pi} \frac{\partial}{\partial z} \int_{y-y_{1}}^{\mathrm{y}-\mathrm{y}_{2}} \int_{0}^{\mathrm{s}_{\mathrm{m}} / \mathrm{r}-1} \frac{\mu(\mathrm{x}-\mathrm{r}-\mathrm{rt}, \mathrm{y}-\mathrm{s}) \mathrm{dtds}}{\sqrt{\mathrm{t}(\mathrm{t}+2)}}
$$

Differentiation leads to

$$
\begin{gathered}
\phi=\frac{z}{2 \pi} \int_{y-y_{1}}^{y-y_{2}} \frac{s_{m} \mu\left(x-s_{m}, y-s\right) d s}{r^{2} \sqrt{s_{m^{2}}-r^{2}}} \\
+\frac{z}{2 \pi} \int_{y-y_{1}}^{y-y_{2}} \int_{0}^{s m^{-r}} \frac{\mu_{x}(x-r-t, y-s)(r+t) d t d s}{r^{2} \sqrt{t(2 r+t)}}
\end{gathered}
$$

The integration with respect to $t$ may be performed by expanding $\mu_{X}$ in powers of $t$ using

$$
I_{n}\left(r, s_{m}\right)=\int_{0}^{s_{m}-r} \frac{t^{n_{d t}}}{\sqrt{t(2 r+t)}}
$$

Hence

$$
\begin{gathered}
\phi=-\frac{\mathrm{z}}{2 \pi} \int_{\mathrm{y}-\mathrm{y}_{2}}^{\mathrm{y}-\mathrm{y}_{1}}\left\{\frac{\mathrm{s}_{\mathrm{m}} \mu\left(\mathrm{x}-\mathrm{s}_{\mathrm{m}}, \mathrm{y}-\mathrm{s}\right)}{\mathrm{r}^{2} \mathrm{R}}+\frac{\mu_{\mathrm{x}}\left(\mathrm{x}_{1} \mathrm{y}-\mathrm{s}\right)\left(\mathrm{s}^{2}-\mathrm{r}^{2}\right)}{\mathrm{r}^{2} \mathrm{R}}\right. \\
\left.-\mu_{\mathrm{xx}}\left[\mathrm{I}_{0}+\frac{\mathrm{s}_{\mathrm{m}}\left(\mathrm{s}_{\mathrm{m}}{ }^{2}-\mathrm{r}^{2}\right)}{\mathrm{r}^{2} \mathrm{R}}\right] / 2\right\} \mathrm{ds}
\end{gathered}
$$


where we have eliminated $I_{2}$ and $I_{1}$ by the recursion formulas in equation (A8). Finally, $\phi$ is obtained by expanding $\mu$ about $\mathrm{x}, \mathrm{y}$ and performing the integration with respect to $\mathrm{s}$. Using the functions defined by equations (A4), (A5), (A10), and (A12) leads to

$$
\begin{gathered}
\phi=-\frac{\mathrm{z}}{2 \pi}\left[\mu \mathrm{Q}_{\mathrm{m} 0}-\mu_{\mathrm{x}} \mathrm{w}_{0}-\mu_{\mathrm{y}} \mathrm{Q}_{\mathrm{m} 1}+\mu_{\mathrm{xx}}\left(\mathrm{w}_{1} / \mathrm{m}+\mathrm{z}^{2} \mathrm{Q}_{\mathrm{m} 0}\right) / 2\right. \\
\left.+\mu_{\mathrm{xy}} \mathrm{w}_{1}+\mu_{\mathrm{yy}}\left(\mathrm{w}_{\mathrm{m} 0}-\mathrm{z}^{2} \mathrm{Q}_{\mathrm{m} 0}\right) / 2\right]
\end{gathered}
$$

The variables $z Q_{m 0}$ and $Q_{m 1}$ may be simplified by discarding those terms which depend only upon the corner point and therefore cancel when integrating around a panel corner. The simpler relation takes the form

$$
\begin{aligned}
\phi= & -\frac{1}{2 \pi}\left[\mu \mathrm{Q}_{\mathrm{I}}-\mu_{\mathrm{X}} \mathrm{zw} 0-\mu_{\mathrm{y}} \mathrm{zw} 0 / \mathrm{m}+\mu_{\mathrm{Xx}} \mathrm{z}\left(\mathrm{w}_{1} / \mathrm{m}+\mathrm{z} \mathrm{Q}_{\mathrm{I}}\right) / 2\right. \\
& \left.+\mu_{\mathrm{xy} \mathrm{zw}_{1}}+\mu_{\mathrm{yy}} \mathrm{z}\left(\mathrm{w}_{\mathrm{m} 0}-\mathrm{zQ}\right) / 2\right]
\end{aligned}
$$

where $\mathrm{zQ}_{\mathrm{mO}}$ has been replaced by the function $\mathrm{Q}_{\mathrm{I}}$ defined in appendix $\mathrm{A}$ by equation (A19) and $Q_{m 1}$ by $w_{0} / m$ in equation (A18). We have also discarded the term $\mathrm{sI}_{0}$ from the function $\mathrm{S}_{\mathrm{o}}$ since it cancels when integration is performed around a panel corner. 


\subsection{PANEL EDGE SINGULARITIES FOR DOUBLET PANELS}

To investigate the singularities which result from discontinuities in doublet strength and its derivatives along panel edges, we consider the potential from the doublet distribution in equation (5.17). The perturbation velocity vector then becomes

$$
\begin{aligned}
\nabla \phi= & -\frac{1}{2 \pi}\left[\mu \nabla \mathrm{Q}_{\mathrm{I}}+\mathrm{Q}_{\mathrm{I}} \nabla \mu-\left(\mu_{\mathrm{x}}+\mu_{\mathrm{y}} / \mathrm{m}\right) \nabla\left(\mathrm{zw}_{0}\right)-\mathrm{zw}_{0} \nabla\left(\mu_{\mathrm{x}}+\mu_{\mathrm{y}} / \mathrm{m}\right)\right. \\
& \left.+\left(\mu_{\mathrm{xx}} / 2 \mathrm{~m}+\mu_{\mathrm{xy}}+\mu_{\mathrm{yy}} / 2 \mathrm{~m}\right) \nabla\left(\mathrm{zw}_{\mathrm{l}}\right)+\mu_{\mathrm{yy}} \nabla\left(\mathrm{zx}_{\mathrm{m}} \mathrm{w}_{0}-\mathrm{z}^{2} \mathrm{Q}_{\mathrm{I}}\right) / 2\right]
\end{aligned}
$$

The contribution to the velocity from a specific panel at a given point $(x, y, z)$ on any panel is found by evaluating the functions $Q_{I}, w_{0}$, and $w_{1}$ and their gradients at the endpoints of each panel edge going around the panel in a counterclockwise direction. For interior points in a network, the induced velocity from a single edge has contributions from the two adjacent panels. If the representations of $\mu$ on the two panels are equal at the panel edge then the term involving $\mu$ in equation (6.1) cancels when the reference point approaches the common boundary. However, a jump in the doublet distribution or in its gradient at a panel edge introduces singularities in the velocily at the panel edge. To investigate these singularities, we study the behavior of $\nabla \mathrm{Q}_{\mathrm{I}}, \nabla\left(\mathrm{zw}_{0}\right)$, and $\nabla\left(\mathrm{zw}_{1}\right)$ as the point $(\mathrm{x}, \mathrm{y}, 0)$ on the panel approaches a point on the panel edge. From equation (A33) for $z=0$, we obtain

$$
\nabla Q_{I}=\left(0,0, \frac{s-s_{m} / m}{x_{m} \sqrt{s_{m^{2}-s^{2}}}}\right)
$$

where $\mathrm{s}_{\mathrm{m}}, \mathrm{s}$ correspond to $\mathrm{x}-\mathrm{x}_{1}, \mathrm{y}-\mathrm{y}_{1}$ or $\mathrm{x}-\mathrm{x}_{2}, \mathrm{y}-\mathrm{y}_{2}$ for each side.

Now $x_{m}$ may be interpreted as the coordinate measuring distance from the panel edge which is defined by $x_{m}=0$. The oblique coordinate is proportional to $y_{m}=s-s_{m} / m$. Thus

$$
\nabla \mathrm{Q}_{\mathrm{I}}=\left(0,0, \mathrm{y}_{\mathrm{m}} / \mathrm{x}_{\mathrm{m}} \sqrt{\mathrm{s}_{\mathrm{m}}^{2}-\mathrm{s}^{2}}\right)
$$

On $\mathrm{z}=0$,

$$
\nabla\left(\mathrm{zw}_{\mathrm{i}}\right)=\left(0,0, \mathrm{w}_{\mathrm{i}}\right)
$$

For points close to subsonic panel edges, we have shown in equation (A31) that

$$
\mathrm{w}_{0} \simeq \frac{\mathrm{m}}{\sqrt{1-\mathrm{m}^{2}}} \log \mathrm{x}_{\mathrm{m}}
$$


and from equation (A9)

$$
w_{1}=-\frac{m^{2} x_{m} \log x_{m}}{\sqrt{\left(1-m^{2}\right)^{3}}}+\frac{m^{2} R}{1-m^{2}}
$$

Note that only $\partial \phi / \partial z$ is singular. Hence, substituting equations (6.2) through (6.5) into equation (6.1) yields for points near the subsonic panel edge on the panel

$$
\phi_{\mathrm{z}}=-\frac{1}{2 \pi}\left\{\mu(\mathrm{x}, \mathrm{y}) \mathrm{y}_{\mathrm{m}} / \mathrm{x}_{\mathrm{m}} \sqrt{\mathrm{s}_{\mathrm{m}}^{2}-\mathrm{s}^{2}}-\left(\mu_{\mathrm{x}} \mathrm{m}+\mu_{\mathrm{y}}\right) \log \mathrm{x}_{\mathrm{m}} / \sqrt{1-\mathrm{m}^{2}}+0(1)\right\}
$$

The oblique coordinates associated with the panel edge represented by $x_{m}=s_{m}-s / m=0$ are defined by

$$
\begin{aligned}
& x^{\prime}=\left(-m s_{m}+s\right) / \sqrt{1-m^{2}} \\
& y^{\prime}=\left(-m s+s_{m}\right) / \sqrt{1-m^{2}}
\end{aligned}
$$

With these coordinates, equation (6.6) may be written

$$
\phi_{\mathrm{z}}=-\frac{1}{2 \pi}\left\{\mu \mathrm{y}^{\prime} / \mathrm{x}^{\prime} \sqrt{\mathrm{s}_{\mathrm{m}}^{2}-\mathrm{s}^{2}}-\left(\partial \mu / \partial \mathrm{x}^{\prime}\right) \log \mathrm{x}^{\prime}+0(1)\right\}
$$

A discontinuity in $\mu$ is seen to introduce a singularity of $1 / \mathrm{x}^{\prime}$ as $\mathrm{x}^{\prime}$ goes to zero near the panel edge $x_{m}=x^{\prime}=0$, while a discontinuity in the conormal derivative $\partial \mu / \partial x^{\prime}$ introduces a logarithmic singularity. As the panel edge approaches a Mach line in direction, the conormal derivative of $\mu$ becomes the tangential derivative, and the $1 / x^{\prime}$ singularity reduces to $1 / \sqrt{x^{\prime}}$, since $y^{\prime}=x^{\prime}$. The continuity of $\mu$ then eliminates both singularities when the panel edge is a Mach line.

For subsonic panel edges these singularities furnish a means of enforcing continuity of $\mu$ and of its conormal derivative at network edges by employing ordinary downwash boundary conditions near the edge of the network. Consider the boundary conditions at two very close points on opposite sides of the network edge. We assume linearized boundary conditions for simplicity in demonstrating the effect of the singularity. At point 1 , we have

$$
\begin{aligned}
\partial \phi / \partial z= & -\frac{1}{2 \pi}\left\{\left[\mu_{1}\left(x_{1}^{\prime}, y_{1}^{\prime}\right)-\mu_{2}\left(x_{1}^{\prime}, y_{1}^{\prime}\right)\right] y_{1}^{\prime} / x_{1}^{\prime} R_{1}\right. \\
& \left.+\left[\mu_{1} x^{\prime}\left(x_{1}^{\prime}, y_{1}^{\prime}\right)-\mu_{2 x^{\prime}}\left(x_{1}^{\prime}, y_{1}^{\prime}\right)\right] \log x_{1}^{\prime}+0(1)\right\}=\left(\partial z / \partial x_{x_{1}^{\prime}}\right.
\end{aligned}
$$


or

$$
\begin{aligned}
& {\left[\mu_{1}\left(\mathrm{x}_{1}^{\prime}, \mathrm{y}_{1}^{\prime}\right)-\mu_{2}\left(\mathrm{x}_{1}^{\prime}, \mathrm{y}_{1}^{\prime}\right)\right] \mathrm{y}_{1}^{\prime} / \mathrm{R}_{1}} \\
& =\left[\mu_{1 \mathrm{x}^{\prime}}\left(\mathrm{x}_{1}^{\prime}, \mathrm{y}_{1}^{\prime}\right)-\mu_{2 \mathrm{x}_{1}^{\prime}}\left(\mathrm{x}_{1}^{\prime}, \mathrm{y}_{1}^{\prime}\right)\right] \mathrm{x}_{1}^{\prime} \log \mathrm{x}_{1}^{\prime}-2 \pi \mathrm{x}_{1}^{\prime}(\partial \mathrm{z} / \partial \mathrm{x}) \mathrm{x}_{1}^{\prime}
\end{aligned}
$$

where $\partial z / \partial x$ is the local streamwise slope of the wing and the subscripts 1 and 2 denote $\mu$ as defined by the parameters associated with the two adjacent panels having the edge in common. The right hand side of equation (6.9) is negligible for $x^{\prime}$ very small and we then approximate the condition

$$
\mu_{1} \simeq \mu_{2}
$$

at one point on the panel edge.

At the second point on the other side of the panel edge, the continuity of $\mu$ by equation (6.9) is satisfied to the order of $x_{m} \log x_{m}$. Then the boundary condition at point 2 is dominated by

$$
\frac{\partial^{\prime}}{\partial \mathrm{x}}\left[\mu_{1}-\mu_{2}\right] \simeq-2 \pi\left(\frac{\partial \mathrm{z}}{\partial \mathrm{x}}\right)_{2} / \log \mathrm{x}^{\prime}
$$

from which we have

$$
\partial \mu_{1} / \partial \mathrm{x}^{\prime} \simeq \partial \mu_{2} / \partial \mathrm{x}^{\prime}
$$

thereby enforcing near continuity of the conormal derivative of $\mu$.

We now consider $\partial \phi / \partial z$ near a supersonic panel edge. For this case, derivatives of $w_{0}, w_{1}$, and $Q_{I}$ vanish ahead of the Mach cone near the panel edge (they actually become imaginary); but $w_{0}, w_{1}, Q_{I}$ take on constant values inside the Mach wedge shown in figure 19. From equations (6.1), (A27), and (A30), we see that on the panel near the supersonic panel edge

$$
\phi_{\mathrm{z}}=-\frac{1}{2 \pi}\left[-\left(\mu_{\mathrm{x}}+\mu_{\mathrm{y}} / \mathrm{m}\right) \mathrm{w}_{0}+\left(\mu_{\mathrm{xx}} / 2 \mathrm{~m}+\mu_{\mathrm{xy}}+\mu_{\mathrm{yy}} / 2 \mathrm{~m}\right) \mathrm{w}_{0}+\mu_{\mathrm{yy}} \mathrm{x}_{\mathrm{m}} \mathrm{w}_{0} / 2\right]_{\mathrm{z}=0}
$$




$$
\phi_{\mathrm{z}}=\frac{1}{4 \sqrt{1-\lambda^{2}}}\left\{-\mu_{\mathrm{x}}-\lambda \mu_{\mathrm{y}}-\left(\mathrm{x}_{\mathrm{m}} / 2\right)\left[\left(\lambda \mu_{\mathrm{xx}}+2 \mu_{\mathrm{xy}}+\lambda \mu_{\mathrm{yy}}\right) \lambda /\left(1-\lambda^{2}\right)-\mu_{\mathrm{yy}}\right]\right\}
$$

For the supersonic leading edge the coordinates along the panel edge and oblique or conormal to the panel edge are

$$
\begin{aligned}
& x^{\prime}=\left(s_{m}-\lambda s\right) / \sqrt{1-\lambda^{2}}=x_{m} / \sqrt{1-\lambda^{2}} \\
& y^{\prime}=\left(-\lambda s_{m}+s\right) / \sqrt{1-\lambda^{2}}
\end{aligned}
$$

Hence

$$
\phi_{\mathrm{Z}}=-\frac{1}{4} \frac{\partial \mu}{\partial \mathrm{x}^{\prime}}+O\left(\mathrm{x}^{\prime}\right)
$$

For a supersonic leading edge, the jump in the conormal derivative of the doublet distribution introduces a jump in the velocity conormal to the plane of the panel.

In addition to the singularities on the panels near the panel edges, there are infinite square root singularities which occur on the Mach cones emanating from panel corners out into the flow field whenever the doublet distribution is not continuous at panel edges. From section A6 of appendix A, it is easily seen that the gradients of the functions have the hyperbolic distance $\mathrm{R}$ in the denominator and hence, become infinite on the Mach cone $\mathrm{R}=0$. If $\mu$ is continuous these singularities are cancelled, providing the edges of two adjacent panels coincide. Although this is not readily apparent from the form of equations (6.1), it is easily shown in section 7 where the line integrals of the doublet distribution along panel edges are discarded in determining the potential and velocity components. 


\subsection{INFLUENCE COEFFICIENTS FOR SUBINCLINED DOUBLET PANELS NEGLECTING THE EDGE VORTEX}

It has been shown that jumps in the doublet distribution across panel edges introduce singularities in the velocity which propagate into the region away from the panel. The original six parameter spline provides a doublet distribution which is almost continuous across panel edges. Since the discontinuity is small, it would appear that the difficulty could be removed by arbitrarily eliminating the line vortex integrals from the velocity aerodynamic influence coefficients. This was quite successful in subsonic flow, but it was found that for supersonic flow even small discontinuities in the doublet at panel edges produced inaccuracies when the line vortex was omitted. For the nine parameter spline, the doublet distribution $\mu$ is continuous and we can therefore simplify the aerodynamic influence coefficients by eliminating all terms involving line integrals of doublet distribution along panel edges. In the influence coefficients previously derived, these integrals are included but introduce no difficulty if the doublet strength is continuous along panel edges. In the following we derive influence coefficients for the flat panel in which line integrals along panel boundaries of the doublet distribution are eliminated. Consider the doublet distribution from one panel and the resulting velocity potential from one edge. This is given by equation (5.14) or

$$
\phi=\frac{1}{2 \pi} \frac{\partial}{\partial z} \int_{y-y_{2}}^{y-y_{1}} \int_{0}^{s_{m} / r-1} \frac{\mu(x-r-r t, y-s) d t d s}{\sqrt{t(t+2)}}
$$

Performing the differentiation yields

$$
\phi=-\frac{z}{2 \pi} \int_{y-y_{2}}^{y-y} 1 \int_{0}^{s_{m}-r} \frac{(r+t) \mu_{x}(x-r-t, y-s) d t d s}{r^{2} \sqrt{t(t+2 r)}}-\frac{z}{2 \pi} \int_{y-y_{2}}^{y-y_{1}} \frac{\mu\left(x-s_{m}, y-s\right) d s}{r^{2} \sqrt{s_{m^{2}}-r^{2}}}
$$

The second term is a line integral of the doublet strength along the edge. Hence, we discard it and obtain

$$
\phi=-\frac{z}{2 \pi} \int_{y-y_{2}}^{y-y_{1}} \int_{0}^{s_{m}-r} \frac{(r+t) \mu_{x}(x-r-t, y-s) d t d s}{r^{2} \sqrt{t(t+2 r)}}
$$


The velocity potential is obtained by expanding $\mu_{\mathrm{X}}$ in powers of $\mathrm{r}+\mathrm{t}$. Thus we obtain

$$
\begin{aligned}
\phi & =-\frac{\mathrm{z}}{2 \pi} \int_{\mathrm{y}-\mathrm{y}_{2}}^{\mathrm{y}-\mathrm{y}_{2}} \int_{0}^{\mathrm{s}_{\mathrm{m}}-\mathrm{r}} \frac{\left[\mu_{\mathrm{x}}(\mathrm{x}, \mathrm{y}-\mathrm{s})(\mathrm{r}+\mathrm{t})-\mu_{\mathrm{xx}}(\mathrm{r}+\mathrm{t})^{2}\right] \mathrm{dtds}}{\mathrm{r}^{2} \sqrt{\mathrm{t}(\mathrm{t}+2)}} \\
& =-\frac{\mathrm{z}}{2 \pi} \int_{\mathrm{y}-\mathrm{y}_{1}}^{\mathrm{y}-\mathrm{y}_{2}}\left[\mu_{\mathrm{x}}(\mathrm{x}, \mathrm{y}-\mathrm{s}) \sqrt{\left.\mathrm{s}^{2}-\mathrm{r}^{2} / \mathrm{r}^{2}-\mu_{\mathrm{xx}}\left(\mathrm{r}^{2} \mathrm{I}_{0}+\mathrm{s}_{\mathrm{m}} \mathrm{R}\right) / 2 \mathrm{r}^{2}\right] \mathrm{ds}}\right.
\end{aligned}
$$

where we made use of the recursion formulas for $I_{n}$ in equation (A8). After integration with respect to $s, \phi$ becomes

$$
\begin{aligned}
\phi & =-\frac{1}{2 \pi}\left[\mu_{\mathrm{X}} \mathrm{P}_{0}-\mu_{\mathrm{Xy}} \mathrm{P}_{1}-\mu_{\mathrm{Xx}}\left(\mathrm{zS}_{0}+\mathrm{P}_{\mathrm{m} 0}\right) / 2\right] \\
& =-\frac{1}{2 \pi}\left[\mu_{\mathrm{X}} \mathrm{P}_{0}-\mu_{\mathrm{xy}} \mathrm{P}_{1}-\mu_{\mathrm{Xx}}\left(2 \mathrm{x}_{\mathrm{m}} \mathrm{w}_{0}-\mathrm{z}^{2} \mathrm{Q}_{\mathrm{I}}+\mathrm{x}_{\mathrm{m}} \mathrm{P}_{0}+\mathrm{P}_{1} / \mathrm{m}\right)\right]
\end{aligned}
$$

where $P_{n}=z \int R s^{n} d s / r^{2}$.

The relation for $\phi$ in equation (7.3) ultimately was not used since it involves the $P_{n}$ functions which are not required for the velocity components. The expression for $\phi$ in cquation (5.17) is valid for continuous $\mu$, introduces no infinite singularities for discontinuous $\mu$, and involves the same functions as the velocity components for continuous $\mu$. Hence, it is more economical to use equation (5.17).

For the perturbation velocity component $\mu$ we have

$$
\phi_{x}=-\frac{z}{2 \pi}\left\{\mu_{x x} \int_{y-y_{2}}^{y-y_{1}} \int_{0}^{s_{m}-r} \frac{(r+t) d t d s}{r^{2} \sqrt{t(t+2 r)}}+\int_{y-y_{2}}^{y-y_{1}} \frac{s_{m} \mu_{x}\left(x-s_{m}, y-s\right) d s}{r^{2} \sqrt{s_{m}^{2}-r^{2}}}\right\}
$$

Integration yields

$$
\phi_{\mathrm{X}}=-\frac{\mathrm{z}}{2 \pi}\left\{\mu_{\mathrm{X}} \mathrm{Q}_{\mathrm{m}_{0}}-\mu_{\mathrm{xy}} \mathrm{w}_{0} / \mathrm{m}-\mu_{\mathrm{Xx}} \mathrm{w}_{0}\right\}
$$


or

$$
\phi_{\mathrm{X}}=-\frac{1}{2 \pi}\left\{\mu_{\mathrm{X}} \mathrm{Q}_{\mathrm{I}}-\mu_{\mathrm{Xy}} \mathrm{zw} 0 / \mathrm{m}-\mu_{\mathrm{Xx}} \mathrm{zw_{0 }}\right\}
$$

For the $y$ component of the perturbation velocity, we have

$$
\begin{aligned}
\phi_{y}=\frac{z}{2 \pi} \int_{y-y_{2}}^{y-y_{1}} \frac{s_{m} \mu_{x}\left(x-s_{m}, y-s\right) d s}{m r^{2} \sqrt{s_{m^{2}-r^{2}}}} & -\frac{z}{2 \pi}\left\{\int_{y-y_{2}}^{y-y} 1 \int_{0}^{s_{m}-r} \frac{(r+t) \mu_{x y} d t d s}{r^{2} \sqrt{t(t+2 r)}}\right. \\
& \left.+\left.\int_{0}^{s_{m}-r} \frac{(r+t) \mu_{x}(x-r-t, y-s) d t}{\sqrt{t(t+2 r)}}\right|_{y-y_{2}} ^{y-y_{1}}\right\}
\end{aligned}
$$

The last term can be discarded since the coefficients of $\mu_{\mathrm{X}}, \mu_{\mathrm{Xy}}$, and $\mu_{\mathrm{XX}}$ from this term depend only upon the endpoints and not on the panel edge slope $m$, and therefore, will cancel in the integration around a panel. The quantity $\mu_{\mathrm{X}}$ in the first integral can be replaced by $\mu_{\mathrm{y}}$ and the derivative along the edge $\frac{\mathrm{d}}{\mathrm{ds}} \mu\left(\mathrm{x}-\mathrm{s}_{\mathrm{m}}, \mathrm{y}-\mathrm{s}\right)$ which will cancel when $\mu$ is continuous on panel edges. We thus obtain

$$
\frac{\mathrm{d}}{\mathrm{ds}} \mu\left(\mathrm{x}-\mathrm{s}_{\mathrm{m}}, \mathrm{y}-\mathrm{s}\right)=-\frac{\mu_{\mathrm{x}}\left(\mathrm{x}-\mathrm{s}_{\mathrm{m}}, \mathrm{y}-\mathrm{s}\right)}{\mathrm{m}}-\mu_{\mathrm{y}}\left(\mathrm{x}-\mathrm{s}_{\mathrm{m}}, \mathrm{y}-\mathrm{s}\right)
$$

and $\phi_{\mathrm{y}}$ becomes

$$
\begin{aligned}
\phi y=-\frac{z}{2 \pi} \int_{y-y_{2}}^{y-y} 1 \frac{s_{m} \mu_{y}\left(x-s_{m}, y-s\right) d s}{r^{2} \sqrt{s_{m^{2}-r^{2}}}} \\
+\frac{z}{2 \pi} \mu_{x y} \int_{y-y_{2}}^{y-y_{1}} \int_{0}^{s_{m^{-r}}} \frac{(r+t) d t d s}{r^{2} \sqrt{t(t+2 r)}}
\end{aligned}
$$

Integration yields

$$
\phi_{\mathrm{y}}=-\frac{\mathrm{z}}{2 \pi}\left\{\mu_{\mathrm{y}} \mathrm{Q}_{\mathrm{m} 0}-\mu_{\mathrm{xy}} \mathrm{Q}_{\mathrm{mm} 0}-\mu_{\mathrm{yy}} \mathrm{Q}_{\mathrm{m}_{1}}+\mu_{\mathrm{xy}}\left[\mathrm{Q}_{\mathrm{mm} 0}-\mathrm{w}_{0}\right]\right\}
$$




$$
\phi_{\mathrm{y}}=-\frac{1}{2 \pi}\left\{\mu_{\mathrm{y}} \mathrm{Q}_{\mathrm{I}}-\mu_{\mathrm{xy}} \mathrm{zw} \mathrm{w}_{0}-\mu_{\mathrm{yy}} \mathrm{zw} 0 / \mathrm{m}\right\}
$$

To find $\phi_{\mathrm{Z}}$ we consider the potential in the original form and obtain

$$
\phi_{\mathrm{z}}=\frac{1}{2 \pi} \frac{\partial^{2}}{\partial \mathrm{z}^{2}} \int_{\mathrm{y}-\mathrm{y}_{2}}^{\mathrm{y}-\mathrm{y} 1} \int_{0}^{\mathrm{s}_{\mathrm{m}}-\mathrm{r}} \frac{\mu(\mathrm{x}-\mathrm{r}-\mathrm{t}, \mathrm{y}-\mathrm{s}) \mathrm{dtd} s}{\sqrt{\mathrm{t}(\mathrm{t}+2 \mathrm{r})}}
$$

Since the operator $\partial^{2} / \partial z^{2}$ equals the operator $\partial^{2} / \partial x^{2}-\partial^{2} / \partial y^{2}$, we may write

$$
\phi_{z}=\frac{1}{2 \pi}\left(\frac{\partial^{2}}{\partial x^{2}}-\frac{\partial^{2}}{\partial y^{2}}\right) \int_{y-y_{2}}^{y-y_{1}} \int_{0}^{s_{m}-r} \frac{\mu(x-r-t, y-s) d t d s}{\sqrt{t(t+2 r)}}
$$

The first integral becomes

$$
\begin{aligned}
& \frac{\partial^{2}}{\partial x^{2}} \int_{y-y_{2}}^{y-y_{1}} \int_{0}^{s_{m}-r} \frac{\mu(x-r-t, y-s) d t d s}{\sqrt{t(t+2 r)}} \\
& =\frac{\partial}{\partial x} \int_{y-y_{2}}^{y-y_{1}} \frac{\mu\left(x-s_{m}, y-s\right) d s}{\sqrt{s_{m^{2}-r^{2}}}} \\
& +\int_{y-y_{2}}^{y-y_{1}} \int_{0}^{s_{m^{-r}} \frac{\mu_{x}(x-r-t, y-s) d t d s}{\sqrt{t(t+2 r)}}} \mid \\
& =\int_{y-y_{2}}^{y-y_{1}} \frac{\mu_{x}\left(x-s_{m}, y-s\right) d s}{\sqrt{s_{m}^{2}-r^{2}}}+\mu_{x x} s_{0}=\mu_{x} w_{0}-\mu_{x y} w_{1}-\mu_{x x}\left(w_{m}-s_{0}\right)
\end{aligned}
$$

Since $S_{0}=\mathrm{sI}_{0}+\mathrm{x}_{\mathrm{m}} \mathrm{w}_{0} \mathrm{Q}_{\mathrm{m} 0}-\mathrm{z}_{\mathrm{m} 0}{ }^{2}$ and $\mathrm{sI}_{0}$ may be discarded as it depends only upon the endpoints, we get for the integral

$$
=\mu_{\mathrm{X}} \mathrm{w}_{0}-\mu_{\mathrm{Xy}} \mathrm{w}_{1}-\mu_{\mathrm{XX}}\left(\mathrm{w}_{1} / \mathrm{m}+\mathrm{zQ}_{\mathrm{I}}\right)
$$


The second term becomes

$$
\begin{aligned}
& \frac{\partial^{2}}{\partial y^{2}} \int_{y-y_{2}}^{y-y_{1}} \int_{0}^{s_{m}-r} \frac{\mu(x-r-t, y-s) d t d s}{\sqrt{t(t+2 r)}}=\left.\frac{\partial}{\partial y} \iint_{0}^{s_{m^{-r}}} \frac{\mu(x-r-t, y-s) d t}{\sqrt{t(t+2 r)}}\right|_{y-y_{2}} ^{y-y_{1}} \\
& \left.\quad+\int_{y-y_{2}}^{y-y_{1}} \int_{0}^{s_{m}-r} \frac{\mu_{y}(x-r-t, y-s) d t d s}{\sqrt{t(t+2 r)}}-\frac{1}{m} \int_{y-y_{2}}^{y-y_{1}} \frac{\mu\left(y-s_{m}, y-s\right) d s}{\sqrt{s_{m^{2}}-r^{2}}}\right\}
\end{aligned}
$$

The last integral on the right hand side of the foregoing equation vanishes because of continuity of $\mu$ on the panel edge and because its coefficients of $\mu_{\mathrm{X}}$, etc. depend only upon the panel endpoints and hence, cancel in the integration around the panel corner. The first integral becomes

$$
\frac{\partial}{\partial y} \int_{y_{1}}^{y_{2}} \frac{\mu\left(x_{1}-\frac{y_{0}-y_{1}}{m}, y_{0}\right) d y_{0}}{\sqrt{\left(x-x_{1}+\frac{y_{0}-y_{1}}{m}\right)^{2}-\left(y-y_{0}\right)^{2}-z^{2}}}
$$

This integral vanishes because of continuity of $\mu$. The remaining integral yields

$$
-\frac{1}{m} \int_{y-y_{2}}^{y-y} 1 \frac{\mu_{y}\left(x-s_{m}, y-s\right) d s}{\sqrt{s_{m^{2}}^{2}-r^{2}}}+\mu_{y y} S_{0}
$$

after discarding terms depending only upon endpoints. Finally, $\phi_{\mathrm{Z}}$ becomes, after combining equations (7.7) and (7.8),

$$
\begin{aligned}
\phi_{\mathrm{z}}= & \frac{1}{2 \pi}\left\{\mu_{\mathrm{x}} \mathrm{w}_{0}-\mu_{\mathrm{xy}} \mathrm{w}_{1}-\mu_{\mathrm{xx}}\left(\mathrm{w}_{1} / \mathrm{m}+\mathrm{zQ}_{\mathrm{I}}\right)\right. \\
& \left.+\mu_{\mathrm{y}} \mathrm{w}_{0} / \mathrm{m}-\mu_{\mathrm{xy}} \mathrm{w}_{\mathrm{m} 0} / \mathrm{m}-\mu_{\mathrm{yy}}\left(\mathrm{s}_{0}+\mathrm{w}_{1} / \mathrm{m}\right)\right\}
\end{aligned}
$$

Since $\mathrm{w}_{\mathrm{mo}} / \mathrm{m}=\mathrm{x}_{\mathrm{m}} \mathrm{w}_{0} / \mathrm{m}+\mathrm{w}_{1} / \mathrm{m}^{2}$ and from the recursion relation in equation (A9).

$$
\mathrm{x}_{\mathrm{m}} \mathrm{w}_{0} / \mathrm{m}=\mathrm{R}-\left(1-\mathrm{m}^{2}\right) \mathrm{w}_{1} / \mathrm{m}^{2}
$$

we see that $\mathrm{w}_{\mathrm{mo}} / \mathrm{m}$ may be replaced by $\mathrm{w}_{1}$ since the term $\mathrm{R}$ may be discarded. Thus, we obtain finally

$$
\begin{aligned}
\phi_{\mathrm{Z}}= & \frac{1}{2 \pi}\left\{\mu_{\mathrm{x}} \mathrm{w}_{0}+\mu_{\mathrm{y}} \mathrm{w}_{0} / \mathrm{m}-2 \mu_{\mathrm{xy}} \mathrm{w}_{1}\right. \\
& \left.-\mu_{\mathrm{xx}}\left(\mathrm{w}_{\mathrm{I}} / \mathrm{m}+\mathrm{z} \mathrm{Q}_{\mathrm{I}}\right)+\mu_{\mathrm{yy}}\left(\mathrm{zQ}_{\mathrm{I}}-\mathrm{w}_{\mathrm{m} 0}\right)\right\}
\end{aligned}
$$




\subsection{AERODYNAMIC INFLUENCE COEFFICIENTS FOR SUBINCLINED SOURCE PANELS}

From equation (3.42) the velocity potential for the source distribution is given by

$$
\phi=-\frac{1}{2 \pi} \int_{\mathrm{S}_{\mathrm{W}}} \frac{\sigma\left(\mathrm{x}_{0}, \mathrm{y}_{0}\right) \mathrm{dx}_{0} \mathrm{dy}_{0}}{\sqrt{\left(\mathrm{x}-\mathrm{x}_{0}\right)^{2}-\left(\mathrm{y}-\mathrm{y}_{0}\right)^{2}-(\mathrm{z}-\zeta)^{2}}}
$$

where $z_{0}=\zeta\left(x_{0}, y_{0}\right)$ is the panel shape, assumed to depart only slightly from the plane $\mathrm{z}_{\mathrm{O}}=0$. The sign of equation (8.1) was chosen opposite from equation (3.42) because we shall use a normal outward from the wing or body into the fluid instead of outward from the fluid region as used in the theory of section 3 . Introducing the variable $t$ as in the doublet potential for integration between an edge and the Mach cone in figure 17 , we obtain the integral

$$
\phi=\frac{1}{2 \pi} \int_{\mathrm{y}_{1}}^{\mathrm{y}_{2}} \int_{0}^{\mathrm{x}-\mathrm{x}_{1}-\mathrm{r}-\left(\mathrm{y}_{0}-\mathrm{y}_{1} \mathrm{i}\right) / \mathrm{m}+\epsilon_{1}} \frac{\sigma\left(\mathrm{x}-\mathrm{r}-\mathrm{t}+\epsilon_{1}, \mathrm{y}_{0}\right) \mathrm{dtdy}_{0}}{\sqrt{\mathrm{t}\left(\mathrm{a}_{1} \mathrm{t}+\mathrm{b}_{1}\right)}}
$$

where $\mathrm{a}_{1}, \mathrm{~b}_{1}$, and $\epsilon_{1}$ are given following equations (5.8). This is easily seen by comparison of the first integral of equations (5.5) and (5.10), respectively.

The integration of the velocity potential including all first order terms in panel curvature, is described in appendix B. Since the terms related to the curvature introduce higher order singularities, we utilize only flat panels in the supersonic panel method. Setting $\zeta=0$ in equation (8.2), we obtain

$$
\phi=\frac{1}{2 \pi} \int_{y-y_{2}}^{y-y_{1}} \int_{0}^{s_{m}-r} \sigma(x-r-t, y-s) d t d s / \sqrt{t(t+2 r)}
$$

Since $\sigma$ is linear, we have

$$
\sigma(\mathrm{x}-\mathrm{r}-\mathrm{t}, \mathrm{y}-\mathrm{s})=\sigma(\mathrm{x}-\mathrm{r}, \mathrm{y}-\mathrm{s})-\sigma_{\mathrm{X}} \mathrm{t}
$$

and $\phi$ becomes, after integration with respect to $t$,

$$
\phi=\frac{1}{2 \pi} \int_{y-y_{2}}^{y-y_{1}}\left[\sigma(x-r, y-s) I_{0}-\sigma_{x} I_{1}\right] d s
$$

where $I_{n}$ is defined in equations (A8) and (A/4) in appendix A. 
Expanding $\sigma$ further yields

$$
\phi=\frac{1}{2 \pi} \int_{y-y_{2}}^{y-y_{1}}\left[\sigma(x, y-s) I_{0}-\sigma_{x} R\right] d s
$$

where we made use of the recursion formula for $\mathrm{I}_{1}$ in equation (A8) of appendix $A$. With the functions defined in equation (A4) of appendix A, the velocity potential finally takes the form

$$
\phi=\frac{1}{2 \pi}\left[\sigma(\mathrm{x}, \mathrm{y}) \mathrm{S}_{0}-\sigma_{\mathrm{x}} \mathrm{R}_{0}-\sigma_{\mathrm{y}} \mathrm{S}_{1}\right]
$$

where the functions $S_{n}$ and $R_{n}$ are defined in equations (A11) and (A12) in appendix $A$.

The velocity potential can be simplified by dropping all terms in $S_{0}, S_{1}$ which depend only upon the endpoints and, hence, cancel in integrating around a panel corner. This leads to

$$
\begin{aligned}
\phi= & \frac{1}{2 \pi}\left\{\sigma(\mathrm{x}, \mathrm{y})\left(\mathrm{x}_{\mathrm{m}} \mathrm{w}_{0}-\mathrm{zQ}_{\mathrm{I}}\right)-\sigma_{\mathrm{x}}\left[\left(\mathrm{x}_{\mathrm{m}}^{2}-\mathrm{z}^{2}\right) \mathrm{w}_{0}\right.\right. \\
& \left.\left.+\mathrm{x}_{\mathrm{m}} \mathrm{w}_{1} / \mathrm{m}\right] / 2-\sigma_{\mathrm{y}}\left(\mathrm{x}_{\mathrm{m}} \mathrm{w}_{1}-\mathrm{z}^{2} \mathrm{w}_{0} / \mathrm{m}\right) / 2\right\}
\end{aligned}
$$

The velocity components are found by taking the gradient of $\phi$ in the form of equation (8.5), namely

$$
\nabla \phi=\frac{1}{2 \pi}\left\{\nabla \sigma \mathrm{S}_{0}+\sigma \nabla \mathrm{S}_{0}-\sigma_{\mathrm{x}} \nabla \mathrm{R}_{0}-\sigma_{\mathrm{y}} \nabla \mathrm{S}_{1}\right\}
$$

From equation (A24),

$$
\nabla \mathrm{S}_{0}=\left(\mathrm{w}_{0}, \mathrm{I}_{0}\left(\mathrm{r}, \mathrm{s}_{\mathrm{m}}-\mathrm{r}\right)-\mathrm{w}_{0} / \mathrm{m},-\mathrm{zQ}_{\mathrm{m} 0}\right)
$$

This may be simplified by discarding terms depending only upon $\mathrm{s}, \mathrm{s}_{\mathrm{m}}$, and $\mathrm{z}$. and using equation (A20). Hence,

$$
\nabla \mathrm{S}_{0}=\left(\mathrm{w}_{0},-\mathrm{w}_{0} / \mathrm{m},-\mathrm{Q}_{\mathrm{I}}\right)
$$


Similarly, with the aid of equations (A24) and (A18), we have

$$
\begin{aligned}
& \nabla \mathrm{S}_{1}=\left(\mathrm{w}_{1},-\mathrm{w}_{1} / \mathrm{m},-\mathrm{zw}_{0} / \mathrm{m}\right) \\
& \nabla \mathrm{R}_{0}=\left(\mathrm{w}_{\mathrm{m} 0},-\mathrm{w}_{\mathrm{m} 0} / \mathrm{m},-\mathrm{zw}_{0}\right)
\end{aligned}
$$

This may be further simplified by noting that

$$
\mathrm{w}_{\mathrm{mo}} / \mathrm{m}=\mathrm{x}_{\mathrm{m}} \mathrm{w}_{0} / \mathrm{m}+\mathrm{w}_{1} / \mathrm{m}^{2}
$$

and, from equation (A9),

$$
\mathrm{w}_{\mathrm{mo}} / \mathrm{m}=\mathrm{R}-\left(1-\mathrm{m}^{2}\right) \mathrm{w}_{1} / \mathrm{m}^{2}
$$

Discarding $\mathrm{R}$, then yields

$$
\nabla \mathrm{R}_{0}=\left(\mathrm{w}_{\mathrm{m0}},-\mathrm{w}_{1},-\mathrm{zw}_{0}\right)
$$

Finally, we obtain the following perturbation velocity vector contributed by one panel edge when evaluated at the endpoints $s=y-y_{1}$ and $s=y-y_{2}$ :

$$
\begin{aligned}
\nabla \phi= & \frac{1}{2 \pi}\left\{\sigma(\mathrm{x}, \mathrm{y})\left(\mathrm{w}_{0},-\mathrm{w}_{0} / \mathrm{m},-\mathrm{Q}_{\mathrm{I}}\right)-\sigma_{\mathrm{x}}\left(\mathrm{zQ}_{\mathrm{I}}+\mathrm{w}_{1} / \mathrm{m},-\mathrm{w}_{1},-\mathrm{zw}_{0}\right)\right. \\
& \left.-\sigma_{\mathrm{y}}\left(\mathrm{w}_{1}, \mathrm{zQ}_{\mathrm{I}}-\mathrm{w}_{\mathrm{m} 0},-\mathrm{zw}_{0} / \mathrm{m}\right)\right\}
\end{aligned}
$$

On the plane $z=0$ which represents the panel, we have

$$
\partial \phi / \partial z=-\sigma(x, y) Q_{I} / 2 \pi= \pm \sigma(x, y) / 2
$$

when the point $x, y$ lies between the ends of the panel edge, and

$$
\partial \phi / \partial \mathrm{z}=0
$$

outside of this region. This is the basis of applying the analysis boundary conditions for the planar wings. The differerice between the upper and lower slopes is seen to be

$$
\partial \phi^{+} / \partial z-\partial \phi^{-} / \partial \mathrm{z}=\sigma(\mathrm{x}, \mathrm{y})
$$

and the source panel represents the thickness distribution for the planar wing. 


\subsection{AERODYNAMIC INFLUENCE COEFFICIENTS FOR SUPERINCLINED PANELS}

\subsection{VELOCITY POTENTIAL FOR SOURCE AND DOUBLET PANELS}

We have seen that to represent solid surfaces, doublet and source panels must be inclined at angles less than the Mach angle. Singularity surfaces for panels at angles with respect to the free stream greater than the Mach angle do exist theoretically, however. These are useful for closing inlets of jet engines and representing boundary conditions at the exit of a jet engine. The intersection of the Mach cone with superinclined planes are circles and ellipses and the integrals of the aerodynamic influence coefficients over a panel must be computed in a somewhat different manner than for the subinclined panels. We shall assume that the panel is aligned normal to the free stream direction. All other inclinations can be treated by the use of a rotation of coordinates and an oblique transformation, which is discussed in Section 10.

The region of integration of the influence coefficients has several special cases. Figure 20a shows the intersection of the Mach cone lying wholly within the panel. No edge or corner is involved in the computation and the velocity potential and velocity take on their simplest form. Figure 20b shows a Macl cone culting one side of the panel, but all corners lie outside the domain of influence. Figure 20c shows one corner of a panel lying inside the intersection circle of the Mach cone. The potential contributed by any panel can be computed by adding and subtracting the integration over the three basic areas illustrated in figure 20 . Consider figure $20 \mathrm{~d}$. The integration over the panel may be found by subtracting, from the basic integration over the circle, the integral over the area bounded by an arc and by a line cutting across the circle as shown by the three sides as extended in figure $20 \mathrm{~d}$. The triangular region formed by extending the two sides of the triangle must then be added since it is subtracted twice.

Another approach is to use polar coordinates in the integrals. By this means it is possible to reduce the integration over a panel to a contour integral over the edge of the area common to both the Mach cone and the panel which we shall designate by $\Sigma$ (see fig. 20 ).

To evaluate the potential for the source and doublet distribution over a panel, we consider the fundamental source potential from which we can obtain the doublet potential and the velocity components by differentiation. This may be expressed in the form

$$
2 \pi \phi_{\mathrm{S}}=\int_{\Sigma} \frac{\mu(\eta, \zeta) \mathrm{dnd} \zeta}{\mathrm{R}_{\mathrm{S}}}, \mathrm{R}_{\mathrm{s}}^{2}=\mathrm{x}^{2}-(\eta-\mathrm{y})^{2}-(\zeta-\mathrm{z})^{2}
$$

Now the singularity distribution $\mu$ expanded about the control point $\mathrm{x}, \mathrm{y}, \mathrm{z}$, becomes

$$
\begin{aligned}
\mu(\eta, \zeta)=\mu(\mathrm{y}, \mathrm{z})+\mu_{\mathrm{y}}(\eta-\mathrm{y})+\mu_{\mathrm{z}}(\zeta-\mathrm{z}) & +\mu_{\mathrm{yy}}(\eta-\mathrm{y})^{2 / 2} \\
& +\mu_{\mathrm{yz}}(\eta-\mathrm{y})(\zeta-\mathrm{z})+\mu_{\mathrm{zz}}(\zeta-\mathrm{z})^{2 / 2}
\end{aligned}
$$


This may be written in vector notation as

$$
\mu(\eta, \zeta)=\mu+\nabla \mu \cdot \mathrm{R}_{1}+\mathrm{R}_{1} \cdot \nabla \nabla \mu \cdot \mathrm{R}_{1} / 2
$$

where $\nabla=\partial / \partial y, \partial / \partial z$ operates only on $\mu$ and $R_{1}$ is the vector $(\eta-y),(\zeta-z)$. Substituting equation (9.2) into equation (9.1) yields

$$
2 \pi \phi_{\mathrm{S}}=\int_{\Sigma}\left[\mu+\nabla \mu \cdot \mathrm{R}_{1}+\mathrm{R}_{1} \cdot \nabla \cdot \nabla \mu \cdot \mathrm{R}_{1} / 2\right] \mathrm{d} \eta \mathrm{d} \zeta / \mathrm{R}_{\mathrm{S}}
$$

now $R_{1} / R_{S}=-\nabla^{\prime} R_{S}$ where $\nabla^{\prime}=\partial / \partial \eta, \partial / \partial \zeta$. Substituting into equation (9.3) yields

$$
\begin{aligned}
2 \pi \phi_{\mathrm{S}} & =\int_{\Sigma}\left[\mu / \mathrm{R}_{\mathrm{S}}-\nabla \mu \cdot \nabla^{\prime} \mathrm{R}_{\mathrm{S}}-\frac{1}{2} \mathrm{R}_{1} \cdot \nabla \nabla \mu \cdot \nabla^{\prime} \mathrm{R}_{\mathrm{S}}\right] \mathrm{d} \eta \mathrm{d} \zeta \\
& =\int_{\Sigma}\left[\mu / \mathrm{R}_{\mathrm{S}}+\nabla \cdot \nabla \mu \mathrm{R}_{\mathrm{S}} / 2-\nabla^{\prime} \cdot\left(\nabla \mu \mathrm{R}_{\mathrm{S}}\right)-\frac{1}{2} \nabla^{\prime} \cdot\left(\mathrm{R}_{1} \cdot \nabla \nabla \mu \mathrm{R}_{\mathrm{S}}\right)\right] \mathrm{d} \eta \mathrm{d} \zeta
\end{aligned}
$$

We now apply the divergence theorem to the last two integrals and obtain

$$
2 \pi \phi_{\mathrm{S}}=\int_{\Sigma}\left[\mu / \mathrm{R}_{\mathrm{S}}+\nabla \cdot \nabla \mu \mathrm{R} / 2\right] \mathrm{d} \eta \mathrm{d} \zeta-\int_{\mathrm{C}_{\Sigma}} \hat{\mathrm{n}} \cdot \nabla \mu \mathrm{R}_{\mathrm{S}} \mathrm{d} l-\int_{\mathrm{C}_{\Sigma}} \mathrm{R}_{1} \cdot \nabla \nabla \mu \cdot \hat{\mathrm{n} \mathrm{R}} \mathrm{d} \mathrm{d} l / 2
$$

where $C_{\Sigma}$ is the contour of $\Sigma$, the surface of the panel enclosed in the Mach cone, and $\hat{n}$ is the outward normal to the contour lying in the plane of $\Sigma$.

We now evaluate the integrals in equation (9.4). Since $\mu$ is constant with respect to the variables of integration, the first integral becomes

$$
\begin{aligned}
\mu \int_{\Sigma} \frac{\mathrm{d} \eta \mathrm{d} \zeta}{\mathrm{R}_{\mathrm{S}}} & =\mu \sum_{\mathrm{K}} \int_{\theta_{\mathrm{K}-1}}^{\theta_{\mathrm{K}}} \mathrm{d} \theta \int_{0}^{\mathrm{r}_{\mathrm{K}}^{(\theta)}} \frac{\mathrm{rdr}}{\sqrt{\mathrm{x}^{2}-\mathrm{r}^{2}}}, \mathrm{r}^{2}=(\eta-\mathrm{y})^{2}+(\xi-\mathrm{z})^{2} \\
& =\mu \sum_{\mathrm{K}} \int_{\theta_{\mathrm{K}-1}}^{\theta_{\mathrm{K}}}\left[\mathrm{x}-\sqrt{\mathrm{x}^{2}-\mathrm{r}_{\mathrm{K}}^{2}(\theta)}\right] \mathrm{d} \theta \\
& =2 \pi \mathrm{C}_{0} \mu \mathrm{x}-\mu \sum_{\mathrm{K}} \int_{\theta_{\mathrm{K}-1}}^{\theta_{\mathrm{K}}} \mathrm{H}_{\mathrm{K}}(\theta) \mathrm{d} \theta
\end{aligned}
$$


where $H_{K}(\theta)=\sqrt{x^{2}-r_{K}^{2}(\theta)}, C_{o}=1$ if the point $y, z$ is inside the panel, and $C_{0}=0$ for points outside. Similarly, the other volume integral becomes

$$
\begin{aligned}
\nabla \cdot \nabla \mu \int_{\Sigma} \mathrm{R}_{\mathrm{S}} \mathrm{d} \eta \mathrm{d} \zeta / 2 & =(\nabla \cdot \nabla \mu / 2) \sum_{\mathrm{K}} \int_{\theta_{\mathrm{K}-1}}^{\theta_{\mathrm{K}}} \int_{0}^{\mathrm{r}_{\mathrm{K}}^{(\theta)}} \sqrt{\mathrm{x}^{2}-\mathrm{r}^{2}} \mathrm{rdrd} \theta \\
& =(\nabla \cdot \nabla \mu / 2) \sum_{\mathrm{K}} \int_{\theta_{\mathrm{K}-1}}^{\theta_{\mathrm{K}}}\left[\mathrm{x}^{3}-\mathrm{H}_{\mathrm{K}}^{3}(\theta)\right] \mathrm{d} \theta / 3 \\
\nabla \cdot \nabla \mu \int \mathrm{R}_{\mathrm{S}} \mathrm{d} \eta \mathrm{d} \zeta / 2 & =\frac{1}{6} \nabla \cdot \nabla \mu\left[2 \pi \mathrm{C}_{0^{\mathrm{x}}}{ }^{3}-\sum_{\mathrm{K}} \int_{\theta_{\mathrm{K}-1}}^{\theta_{\mathrm{K}}} \mathrm{H}_{\mathrm{K}}^{3}(\theta) \mathrm{d} \theta\right]
\end{aligned}
$$

We have now reduced the potential $\phi_{\mathrm{S}}$ to a sum of contour integrals around the area $\Sigma$ of the panel contained inside the Mach cone. Since $\mathrm{R}_{\mathrm{S}}$ (or $\mathrm{H}$ ) vanishes on the cone, the line integrals reduce to integrals over the panel edges contained inside the Mach cone.

We now evaluate the integration over each edge. For this it is convenient to use coordinates oriented normal and tangent to each panel edge as shown in figures 21 and 22 . It is seen that the angle $\theta$ in terms of these coordinates is given by

$$
\theta=\tan ^{-1}\left(\frac{0-y_{c}}{z_{c}-\zeta}\right)=\tan ^{-1}\left(\frac{y_{c}}{\zeta-z_{c}}\right)
$$

from which

$$
\mathrm{d} \theta=-\mathrm{y}_{\mathrm{c}} \mathrm{d} \zeta / \mathrm{r}^{2}, \quad \mathrm{r}^{2}=\mathrm{y}_{\mathrm{e}}{ }^{2}+\left(\zeta-\mathrm{z}_{\mathrm{e}}\right)^{2}
$$


The derivatives of $\mu$ in the contour integrals of equation (9.4) become

$$
\nabla \mu \cdot \hat{n}=\mu_{y_{e}}
$$

and

$$
\mathrm{R}_{1} \cdot \nabla \nabla \mu \cdot \hat{\mathrm{l}_{i}}=\left(\zeta-\mathrm{z}_{\mathrm{e}}\right) \mu_{\mathrm{y}_{\mathrm{e}} \mathrm{z}_{\mathrm{e}}}-\mathrm{y}_{\mathrm{e}} \mu_{\mathrm{y}_{\mathrm{e}} \mathrm{y}_{\mathrm{e}}}
$$

Then the last integral of equation ( 9.4$)$ becomes, for each edge,

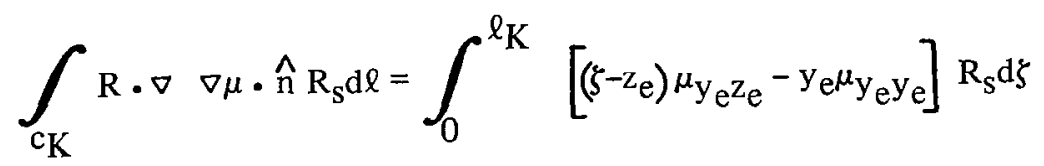

$$
\begin{aligned}
& =\left.\mu_{y_{e} z_{e}} R_{s}^{3 / 3}\right|_{0} ^{\ell_{K}}-y_{e} \mu_{y_{e} y_{e}} \int_{0}^{\ell_{K}} R_{s} d \zeta
\end{aligned}
$$

since $R_{s}^{2}=x^{2}-\left(\zeta-z_{e}\right)^{2}-y_{e}^{2}$

We also have

$$
\begin{aligned}
& \int_{\theta_{\mathrm{K}-1}}^{\theta_{\mathrm{K}}} \mathrm{H}_{\mathrm{K}}(\theta) \mathrm{dt}=-\mathrm{y}_{\mathrm{e}} \int_{0}^{\ell_{\mathrm{K}}} \mathrm{R}_{\mathrm{s}} \mathrm{d} \zeta / \mathrm{r}^{2} \\
& \int_{\theta_{\mathrm{K}-1}^{\theta_{\mathrm{K}}} \mathrm{H}_{\mathrm{K}}^{3}(\theta) \mathrm{d} \theta=-\mathrm{y}_{\mathrm{e}} \mathrm{x}^{2} \int_{0}^{\ell_{\mathrm{K}}} \mathrm{R}_{\mathrm{s}} \mathrm{d} \zeta / \mathrm{r}^{2}+\mathrm{y}_{\mathrm{e}} \int_{0}^{\ell_{\mathrm{K}}} \mathrm{R}_{\mathrm{s}} \mathrm{d} \zeta}^{\int_{\nabla \mu} \hat{\mathrm{n}} \mathrm{Hd} \ell=\mu_{\mathrm{y}_{\mathrm{e}}} \int_{0}^{\ell_{\mathrm{K}}} \mathrm{R}_{\mathrm{s}} \mathrm{d} \xi}
\end{aligned}
$$

Utilizing equations $(9.5),(9.7),(9.8)$, and (9.9) in equation (9.4) yields the potential

$$
\phi_{\mathrm{S}}=\mathrm{C}_{0}\left[\mu \mathrm{x}+\mathrm{x}^{3} \nabla \cdot \nabla \mu / 6\right]+\sum_{\mathrm{k}} \phi_{\mathrm{sk}}
$$


where

$$
\begin{aligned}
& 2 \pi \phi_{\mathrm{sk}}=\mu_{\mathrm{y}} \int_{0}^{\ell} \mathrm{R}_{\mathrm{s}} \mathrm{d} \zeta / \mathrm{r}^{2}+\nabla \cdot \nabla \mu\left[\mathrm{y}_{\mathrm{e}^{\mathrm{x}^{2}}} \int_{0}^{\ell} \mathrm{R}_{\mathrm{s}} \mathrm{d} \zeta / \mathrm{r}^{2}-\mathrm{y}_{\mathrm{e}} \int_{0}^{\ell} \mathrm{R}_{\mathrm{s}} \mathrm{d} \zeta\right] / 6 \\
& -\mu_{y_{e}} \int_{0}^{\ell} R_{s} d \zeta+\mu_{y_{e} z_{e}} R_{s} 3 / 6 \prod_{0}^{\ell}+y_{e} \mu_{y_{e}} y_{e} \int_{0}^{\ell} R_{s} d \zeta / 2
\end{aligned}
$$

Now since the doublet potential $\phi_{\mathrm{d}}$ is given by differentiating $\phi_{\mathrm{S}}$ with respect to $\mathrm{x}$ we obtain

$$
\left.\phi_{\mathrm{d}}=\mathrm{C}_{0} \bar{L}^{\mu+\mathrm{x}^{2}} \nabla \cdot \nabla \mu / 2\right]+\sum_{\mathrm{k}} \phi_{\mathrm{k}}
$$

where

$$
\begin{aligned}
& 2 \pi \phi_{\mathrm{dk}}=\mu x y_{\mathrm{e}} \int_{0}^{\ell} \mathrm{d} \zeta / \mathrm{r}^{2} \mathrm{R}_{\mathrm{s}}+\nabla \cdot \nabla \mu\left[\mathrm{x}^{3} \mathrm{y}_{\mathrm{e}} \int_{0}^{\ell} \mathrm{d} \zeta / \mathrm{r}^{2} \mathrm{R}_{\mathrm{s}}-\mathrm{xy} \mathrm{y}_{\mathrm{e}} \int_{0}^{\ell} \mathrm{d} \zeta / \mathrm{R}_{\mathrm{s}}\right. \\
& \left.+2 x y_{e} \int_{0}^{\ell} R_{s} \mathrm{~d} \zeta / \mathrm{r}^{2}\right] / 6-\mu_{y_{\mathrm{e}}} \mathrm{x} \int_{0}^{\ell}
\end{aligned}
$$

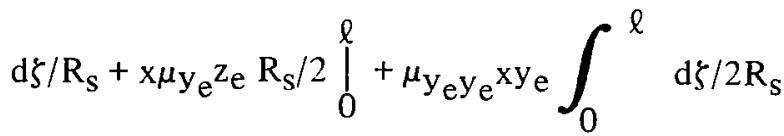

We can express all integrals in terms of two basic functions. Now

$$
y_{e} \int_{0}^{\ell} R_{s} d \xi / r^{2}=x^{2} y_{e} \int_{0}^{\ell} d \xi / r^{2} R_{s}-y_{e} \int_{0}^{\ell} d \xi / R_{s}
$$

61 
We define the basic functions as

$$
\widetilde{Q}_{I}=x y_{e} \int_{0}^{\ell} d \xi / r^{2} R_{s}, \widetilde{w}_{n}=\int_{0}^{\ell} \xi^{n} d \xi / R_{s}
$$

The functions $\widetilde{Q}_{i}$ and $\widetilde{w}_{n}$ are integrated in section $A 9$ of appendix $A$.

The doublet potential then can be written

$$
\phi_{\mathrm{dk}}=\frac{1}{2 \pi}\left\{\mu \widetilde{\mathrm{Q}}_{\mathrm{I}}-\mathrm{x} \mu_{\mathrm{y}_{\mathrm{e}}} \widetilde{\mathrm{w}}_{0}+\mathrm{x} \mu_{\mathrm{y}_{\mathrm{e}} \mathrm{z}_{\mathrm{e}}} \mathrm{R} / 2-\mathrm{xy}_{\mathrm{e}} \mu_{\mathrm{z}_{\mathrm{e}} \mathrm{z}_{\mathrm{e}}} \widetilde{\mathrm{w}}_{0} / 2+\mathrm{x}^{2} \widetilde{\mathrm{Q}}_{\mathrm{I}}\left(\mu_{\mathrm{yy}}+\mu_{\mathrm{zz}}\right) / 2\right\}
$$

since $\nabla \cdot \nabla \mu$ is invariant with rotation of coordinates.

The source potential is found from equation $(9.10)$ by neglecting the quadratic terms. We obtain

$$
\phi_{\mathrm{S}}=c_{0} \sigma \mathrm{x}+\sum_{\mathrm{k}} \phi_{\mathrm{sk}}
$$

where

$$
\phi_{\mathrm{sk}}=\sigma \mathrm{x} \widetilde{\mathrm{Q}}_{\mathrm{I}}-\sigma_{\mathrm{y}_{\mathrm{e}}}\left[\left(\mathrm{x}^{2}-\mathrm{y}_{\mathrm{e}}^{2-\mathrm{z}_{\mathrm{e}}}\right)^{2} \breve{\mathrm{v}}_{0}+2 \mathrm{z}_{\mathrm{e}} \widetilde{\mathrm{w}}_{1}-\widetilde{\mathrm{w}}_{2}\right]
$$

The functions $\widetilde{w}_{2}$ and $\widetilde{w}_{1}$ can be expressed in terms of $\widetilde{w}_{0}$ by using the recursion relations for $\widetilde{w}_{\mathrm{n}}$ in section $\mathrm{A} 9$ of appendix $\mathrm{A}$. This leads to

$$
\phi_{\mathrm{sk}}=\sigma \mathrm{x} \widetilde{\mathrm{Q}}_{\mathrm{I}}-\sigma_{\mathrm{y}_{\mathrm{e}}}\left[\left(\mathrm{x}^{2}-\mathrm{y}_{\mathrm{e}}{ }^{2}\right) \widetilde{\mathrm{w}}_{0}+\left(\zeta-\mathrm{z}_{\mathrm{e}}\right) \mathrm{R}_{\mathrm{s}}\right] / 2
$$




\subsection{VELOCITY COMPONENTS FOR THE DOUBLET DISTRIBUTION WITHOUT EDGE VORTICES}

When the line integral of the doublet strength is discarded from the integration over the panel, the velocity vector is given by the formula

$$
2 \pi \overline{\bar{V}}=\int_{\Sigma}^{*}\left[\hat{n} \times \nabla^{\prime} \mu\right] \times \widetilde{\nabla} \psi d \eta d \zeta
$$

where

$$
\begin{gathered}
\psi=1 / \mathrm{R}_{\mathrm{S}}, \mathrm{R}_{\mathrm{s}}{ }^{2}=(\xi-\mathrm{x})^{2}-(\eta-\mathrm{y})^{2}-(\xi-\mathrm{z})^{2} \\
\nabla^{\prime}=\partial / \partial \xi, \partial / \partial \eta, \partial+\partial \xi
\end{gathered}
$$

and

$$
\widetilde{\nabla}=-\partial / \partial \xi, \partial / \partial \eta, \partial / \partial \zeta
$$

The asterisk denotes finite part of the integral in the sense of Hadamard [11], since the integral does not exist in the usual sense. Equation (9.17) can be written in the following form after expanding the triple cross product:

$$
2 \pi \bar{V}=\int_{\Sigma}^{*}\left[\hat{n} \cdot \widetilde{\nabla} \psi \nabla^{\prime} \mu-\hat{n} \nabla^{\prime} \mu \cdot \widetilde{\nabla} \psi\right] \mathrm{d} \eta \mathrm{d} \zeta
$$

We assume all of the surface $\Sigma$ lies in the plane $\mathrm{x}=0$. Then $\hat{\mathrm{n}}=(1,0,0), \mu=\mu(\eta, \zeta)$, and we have for the $\mathrm{x}$ component of velocity

$$
2 \pi \mathrm{u}=-\int_{\Sigma}^{*} \nabla^{\prime} \mu \cdot \tilde{\nabla} \psi \mathrm{d} \eta \mathrm{d} \zeta=-\int_{\Sigma}^{*} \nabla^{\prime} \mu \cdot \nabla^{\prime} \psi \mathrm{d} \eta \mathrm{d} \xi
$$

where now,

$$
\nabla^{\prime}=(\partial / \partial \eta, \partial / \partial \zeta)
$$


For the components lying in the plane of $\Sigma$ we have, since $\partial / \partial \xi=-\partial / \partial x$

$$
2 \pi \overrightarrow{\mathrm{V}}=\int_{\Sigma}^{*} \nabla^{\prime} \mu \frac{\partial \psi}{\partial \mathrm{x}} \mathrm{d} \eta \mathrm{d} \zeta
$$

Now equation (9.18) may be written in the form

$$
2 \pi \mathrm{u}=-\int_{\Sigma}^{*} \nabla^{\prime} \mu \cdot \nabla^{\prime} \psi \mathrm{d} \eta \mathrm{d} \xi=-\int_{\Sigma}^{*}\left[\nabla^{\prime} \cdot\left(\nabla^{\prime} \mu \psi\right)-\nabla^{\prime} \cdot \nabla^{\prime} \mu \psi\right] \mathrm{d} \eta \mathrm{d} \xi
$$

Application of the divergence theorem to the first integral yields

$$
2 \pi \mathrm{u}=-\int_{C_{\Sigma}}^{*} \nabla^{\prime} \mu \cdot \hat{\mathrm{n}} \psi \mathrm{d} \ell+\int_{\Sigma}^{*} \nabla^{\prime} \cdot \nabla^{\prime} \mu \psi \mathrm{d} \eta \mathrm{d} \xi
$$

where $\hat{n}$ now denotes the normal of the contour in the plane of the panel. To express the integral in a form which can be integrated without knowing the exact coefficients of $\mu$, we expand about the control point projection $\mathrm{y}, \mathrm{z}$ and obtain

$$
\mu(\eta, \zeta)=\mu(\mathrm{y}, \mathrm{z})+(\eta-\mathrm{y}) \mu_{\mathrm{y}}+(\zeta-\mathrm{z}) \mu_{\mathrm{z}}+\mu_{\mathrm{yy}}(\eta-\mathrm{y})^{2} / 2+\mu_{\mathrm{yz}}(\eta-\mathrm{y})(\zeta-\mathrm{z})+\mu_{\mathrm{zz}}(\zeta-\mathrm{z})^{2} / 2
$$

For the gradient we have

$$
\nabla^{\prime} \mu=\left[\mu_{\mathrm{y}}+\mu_{\mathrm{yy}}(\mathrm{n}-\mathrm{y})+\mu_{\mathrm{yz}}(\zeta-\mathrm{z})\right],\left[\mu_{\mathrm{z}}+\mu_{\mathrm{yz}}(\eta-\mathrm{y})+\mu_{\mathrm{zz}}(\zeta-\mathrm{z})\right]
$$

or in vector notation

$$
\nabla^{\prime} \mu=\left(\mu_{\mathrm{y}}+\nabla \mu_{\mathrm{y}} \cdot \mathrm{R}_{1}, \mu_{\mathrm{z}}+\nabla \mu_{\mathrm{z}} \cdot \mathrm{R}_{1}\right)
$$

where $\mathrm{R}_{1}=(\eta-\mathrm{y}, \zeta-\mathrm{z}) \quad$ and $\quad \nabla=(\partial / \partial \mathrm{y}, \partial / \partial \mathrm{z})$.

We also have

$$
\nabla^{\prime} \mu \cdot \hat{n}=\mu_{\mathrm{y}} \mathrm{n}_{\mathrm{y}}+\mu_{\mathrm{z}} \mathrm{n}_{\mathrm{z}}+\mathrm{n}_{\mathrm{y}} \nabla \mu_{\mathrm{y}} \cdot \mathrm{R}_{1}+\mathrm{n}_{\mathrm{z}} \nabla \mu_{\mathrm{z}} \cdot \mathrm{R}_{1}
$$


For straight edges, $\mathrm{n}_{\mathrm{y}}$ and $\mathrm{n}_{\mathrm{z}}$ are constants and can be moved inside the $\nabla$ operator to yield

$$
\nabla^{\prime} \mu \cdot \hat{n}=\mu_{n}+\nabla \mu_{n} \cdot R_{1}
$$

also

$$
\nabla^{\prime} \cdot \nabla^{\prime} \mu=\mu_{\mathrm{yy}}+\mu_{\mathrm{zz}}=\nabla \cdot \nabla \mu
$$

Equation (9.20) becomes, after substituting equations (9.21) and (9.22)

$$
2 \pi \mathrm{u}=-\int_{C_{\Sigma}}^{*}\left[\mu_{\mathrm{n}}+\nabla \mu_{\mathrm{n}} \cdot \mathrm{R}_{1}\right] \psi \mathrm{d} \ell+\nabla \cdot \nabla \mu \int_{\Sigma} \psi \mathrm{d} \eta \mathrm{d} \zeta
$$

Similarly, equation (9.19) yields

$$
\begin{array}{r}
2 \pi \mathrm{v}=\frac{\partial}{\partial \mathrm{x}} \int_{\Sigma}\left[\mu_{\mathrm{y}}+\nabla \mu_{\mathrm{y}} \cdot \mathrm{R}_{1}\right] \psi \mathrm{d} \eta \mathrm{d} \zeta \\
2 \pi \mathrm{w}=\frac{\partial}{\partial \mathrm{x}} \int_{\Sigma}\left[\mu_{\mathrm{z}}+\nabla \mu_{\mathrm{z}} \cdot \mathrm{R}_{1}\right] \psi \mathrm{d} \eta \mathrm{d} \zeta
\end{array}
$$

where integration is to be performed first.

Except for the first derivatives with respect to $y$ and $z$ of $\mu$ in equations (9.24) and (9.25), the integrals are expressed in a form independent of coordinate system. To evaluate the line integrals we introduce coordinates related to each edge. First, we perform one in tegration of the area integrals.

$$
\int_{\Sigma}^{*} \psi \mathrm{d} \eta \mathrm{d} \zeta=\sum_{\mathrm{k}} \int_{\theta}^{*} \theta_{\mathrm{K}-1} \int_{0}^{\mathrm{r}_{\mathrm{K}}^{(\theta)}} \operatorname{rdrd} \theta / \sqrt{\mathrm{x}^{2}-\mathrm{r}^{2}}
$$


where $\theta_{\mathrm{k}}$ and $\theta_{\mathrm{k}-1}$ are the angles, the kth edge of $\Sigma$ makes with the Mach cone center $\mathrm{y}, \mathrm{z}$. Intcgrating equation (9.26) yields from equation (9.5).

$$
\int_{\Sigma}^{*} \psi \mathrm{d} \eta \mathrm{d} \zeta=2 \pi \mathrm{C}_{0} \mathrm{x}-\sum_{\mathrm{K}} \int_{\theta_{\mathrm{K}-1}}^{\theta_{\mathrm{K}}} \mathrm{H}_{\mathrm{K}}(\theta) \mathrm{d} \theta
$$

where $\mathrm{C}_{0}=1$ if $\mathrm{y}, \mathrm{z}$ lies inside $\Sigma$ and 0 otherwise.

Since $\mathrm{R}_{1} \psi=-\nabla^{\prime} \mathrm{R}_{\mathrm{S}}$, equation (9.24) becomes

$$
2 \pi \mathrm{v}=\frac{\partial}{\partial \mathrm{x}}\left\{\mu_{\mathrm{y}} \int_{\Sigma} \psi \mathrm{d} \eta \mathrm{d} \zeta-\int_{\Sigma} \nabla \mu_{\mathrm{y}} \cdot \nabla^{\prime} \mathrm{R}_{\mathrm{s}} \mathrm{d} \eta \mathrm{d} \zeta\right\}
$$

Since $\mu$ does not depend on $\eta$ and $\zeta$, we have

$$
2 \pi \mathrm{v}=\frac{\partial}{\partial \mathrm{x}}\left\{\mu_{\mathrm{y}} \int_{\Sigma} \psi \mathrm{d} \eta \mathrm{d} \zeta-\int_{\Sigma} \nabla^{\prime} \cdot\left(\nabla \mu_{\mathrm{y}} \mathrm{R}_{\mathrm{s}}\right) \mathrm{d} \eta \mathrm{d} \zeta\right\}
$$

Applying the divergence theorem to the second integral yields

$$
\begin{aligned}
2 \pi \mathrm{v}= & \frac{\partial}{\partial \mathrm{x}}\left\{\mu_{\mathrm{y}} \int_{\Sigma} \psi \mathrm{d} \eta \mathrm{d} \zeta-\int_{\mathrm{C}_{\Sigma}} \nabla \mu_{\mathrm{y}} \cdot \hat{\mathrm{n}} \mathrm{R}_{\mathrm{S}} \mathrm{d} \ell\right\} \\
& =\frac{\partial}{\partial \mathrm{x}}\left[\mu_{\mathrm{y}} \int_{\Sigma} \psi \mathrm{d} \eta \mathrm{d} \zeta\right]-\mathrm{x} \int_{\mathrm{C}_{\Sigma}} \nabla \mu_{\mathrm{y}} \cdot \hat{\mathrm{n}} \mathrm{d} \ell / \mathrm{R}_{\mathrm{S}}
\end{aligned}
$$

From equation (9.27)

$$
\begin{aligned}
& 2 \pi \mathrm{v}=\mu_{\mathrm{y}}\left[2 \pi \mathrm{C}_{0}-\mathrm{x} \quad \sum_{\mathrm{K}} \int_{\theta_{\mathrm{K}-1}}^{* \theta_{\mathrm{K}}} \mathrm{d} \theta / \mathrm{H}_{\mathrm{K}}(\theta)\right] \\
& -x \int_{C_{\Sigma}}^{*} \nabla \mu_{\mathrm{y}} \cdot \hat{n} \mathrm{~d} \ell / \mathrm{R}_{\mathrm{S}}
\end{aligned}
$$


Similarly, equation ( 9.23$)$ becomes

$$
2 \pi \mathrm{u}=\nabla \cdot \nabla \mu\left[2 \pi \mathrm{C}_{0} \mathrm{x}-\sum_{\mathrm{K}} \int_{\theta_{\mathrm{K}-1}}^{\theta_{\mathrm{K}}} \mathrm{H}_{\mathrm{K}}(\theta) \mathrm{d} \theta\right]-\int_{\mathrm{C}_{\Sigma}}^{*}\left[\mu_{\mathrm{n}}+\nabla \mu_{\mathrm{n}} \cdot \mathrm{R}_{1}\right] \psi \mathrm{d} \ell
$$

The portion of the contour which are arcs of the Mach cone do not contribute to the finite part of the integrals since the integrand is infinite on the Mach cone. Restricting the integrations over the straight segments allows us to remove the finite part since now the integrals exist in the usual mathematical sense. Since $R_{s}^{2}=x^{2}-r^{2}$, we can write

$$
\begin{aligned}
\int_{\theta_{\mathrm{K}-1}}^{\theta} \mathrm{K} \mathrm{H}_{\mathrm{K}}(\theta) \mathrm{d} \theta=-\mathrm{y}_{\mathrm{e}} \int_{0}^{\ell} \mathrm{R}_{\mathrm{s}} \mathrm{d} \zeta / \mathrm{r}^{2} & =-\mathrm{y}_{\mathrm{e}} \mathrm{x}^{2} \int_{0}^{\ell} \frac{\mathrm{d} \zeta}{\mathrm{r}^{2} \mathrm{R}_{\mathrm{s}}}+\mathrm{y}_{\mathrm{e}} \int_{0}^{\ell} \frac{\mathrm{d} \zeta}{\mathrm{R}_{\mathrm{S}}} \\
& =-\mathrm{x} \widetilde{\mathrm{Q}}_{\mathrm{I}}+\mathrm{y}_{\mathrm{e}} \widetilde{\mathrm{w}}_{0}
\end{aligned}
$$

Since $\mu_{\mathrm{n}}=\mu_{\mathrm{y}_{\mathrm{e}}}$,

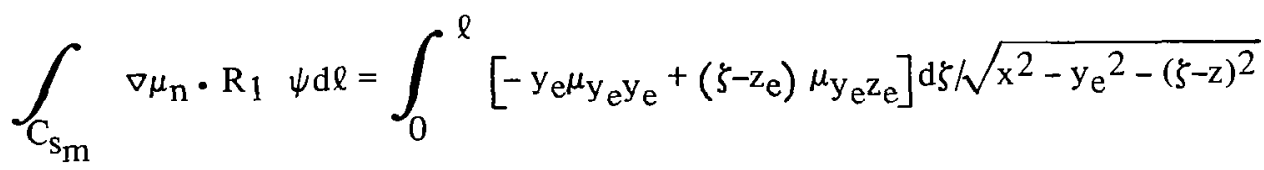

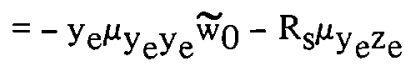

Finally, the velocity components can be written

$$
\begin{aligned}
& \mathrm{u}=\mathrm{x} \nabla \cdot \nabla \mu \mathrm{C}_{0}+\underset{\mathrm{k}}{\Sigma} \mathrm{u}_{\mathrm{k}} \\
& \mathrm{v}=\mu_{\mathrm{y}} \mathrm{C}_{0}+\underset{\mathrm{k}}{\sum} \mathrm{v}_{\mathrm{k}} \\
& \mathrm{w}=\mu_{\mathrm{z}} \mathrm{C}_{0}+\underset{\mathrm{k}}{\sum} \mathrm{w}_{\mathrm{k}}
\end{aligned}
$$


where

$$
\begin{aligned}
& u_{k}=\left[-\mu_{y_{e}} \widetilde{w}_{0}-y_{e} \mu_{z_{e} z_{e}} \widetilde{w}_{0}+\mu_{y_{e} z_{e}} R_{s}+x \widetilde{Q}_{I \nabla \cdot \nabla \mu}\right] / 2 \pi \\
& v_{\mathrm{k}}=\left[\mu_{\mathrm{y}} \widetilde{\mathrm{Q}}_{\mathrm{I}}-\mathrm{x} \mu_{\mathrm{yy}} \widetilde{\mathrm{w}}_{0}\right] / 2 \pi \\
& \mathrm{w}_{\mathrm{k}}=\left[\mu_{\mathrm{z}} \widetilde{\mathrm{Q}}_{\mathrm{I}}-\mathrm{x} \mu_{\mathrm{zy}_{\mathrm{e}}} \widetilde{\mathrm{w}}_{0}\right] / 2 \pi
\end{aligned}
$$

\subsection{VELOCITY COMPONENTS FOR SOURCE DISTRIBUTION}

The velocity components from the source distribution on a superinclined panel are obtained by integrating the gradient of equation (9.1) or

$$
2 \pi \overline{\mathrm{V}}=\nabla \int_{\Sigma} \sigma(\eta, \zeta) \psi \mathrm{d} \eta \mathrm{d} \zeta
$$

where $\nabla=\partial / \partial \mathrm{x}, \partial / \partial \mathrm{y}, \partial / \partial \mathrm{z}$.

It is convenient to separate the velocity vector into components $u$ and $\bar{V}_{p}$, where $\bar{V}_{p}$ is the velocity vector in the plane of $\Sigma$. We easily see that

$$
\mathrm{u}=\frac{1}{2 \pi} \frac{\partial}{\partial \mathrm{x}} \int_{\Sigma} \sigma \psi \mathrm{d} \eta \mathrm{d} \zeta=\phi_{\mathrm{d}}
$$

where $\phi_{\mathrm{d}}$ is the potential for the doublet after dropping the quadratic terms. Now $\overline{\mathrm{V}}_{\mathrm{p}}$ may be written

$$
\begin{aligned}
2 \pi \overline{\mathrm{V}}_{\mathrm{p}} & =-\int_{\Sigma}^{*} \sigma(\eta, \zeta) \nabla^{\prime} \psi \mathrm{d} \eta \mathrm{d} \zeta \\
& =-\int_{\Sigma}^{*} \nabla^{\prime}(\sigma \psi) \mathrm{d} \eta \mathrm{d} \zeta+\int_{\Sigma} \nabla^{\prime} \sigma \psi \mathrm{d} \eta \mathrm{d} \zeta
\end{aligned}
$$

where $\nabla^{\prime}=(\partial / \partial \eta, \partial / \partial \xi)$.

Applying the divergence theorem to the first term yields

$$
2 \pi \overline{\mathrm{V}}_{\mathrm{p}}=-\int_{C_{\Sigma}}^{*} \hat{\mathrm{n}} \sigma(\eta, \zeta) \psi \mathrm{d} \ell+\nabla \sigma \int_{\Sigma} \psi \mathrm{d} \eta \mathrm{d} \zeta
$$


Here we have used the property that $\sigma$ is linear.

Now the curved parts of the contour make no contribution to the finite part. Restricting the contour integration to the straight segments allows us to eliminate the finite part since the remaining integrals exist. In terms of the coordinates $y_{e}, z_{e}$ associated with the edge, the expansion of the source distribution $\sigma$ takes the form

$$
\sigma(\eta, \zeta)=\sigma\left(\mathrm{y}_{\mathrm{e}}, \mathrm{z}_{\mathrm{e}}\right)+\sigma_{\mathrm{y}_{\mathrm{e}}}\left(0-\mathrm{y}_{\mathrm{e}}\right)+\sigma_{\mathrm{z}_{\mathrm{e}}}\left(\zeta-\mathrm{z}_{\epsilon}\right)
$$

Then the first integral in equation (9.35) becomes, for each straight segment

$$
\begin{gathered}
-\left(\sigma-\sigma_{y_{e} y_{e}}\right) \hat{n}_{k} \int_{0}^{\ell} \frac{d \zeta}{\sqrt{x^{2}-y_{e}^{2}-\left(\zeta-z_{e}\right)^{2}}}-\sigma_{z_{c}} \hat{n}_{k} \int_{0}^{\ell} \frac{\left(\zeta-z_{e}\right) d \zeta}{\sqrt{x^{2}-y_{e}^{2}-\left(\zeta-z_{e}\right)^{2}}} \\
=\left[-\left(\sigma-\sigma y_{e} y_{e}\right) \widetilde{w}_{0}+\sigma_{z_{e}} R_{s}\right] \hat{n}_{k}
\end{gathered}
$$

where functions $\widetilde{w}_{0}$ and $R_{S}$ are evaluated at the endpoints of the side, or its intersection with the Mach cone.

The second integral in equation (9.35) has already been evaluated in equation (9.27) and (9.29). The $u$ component is found from equations (9.12) and (9.16) after dropping the quadratic terms. Hence, the velocity components take the form

$$
\begin{aligned}
& \mathrm{u}=\mathrm{C}_{0} \sigma+\sum_{\mathrm{k}} \mathrm{u}_{\mathrm{k}} \\
& \overline{\mathrm{v}}_{\mathrm{p}}=\mathrm{C}_{0} \nabla \sigma+\sum_{\mathrm{k}} \overline{\mathrm{V}}_{\mathrm{pk}}
\end{aligned}
$$

where

$$
\begin{aligned}
& \mathrm{u}_{\mathrm{k}}=\left[\sigma \widetilde{\mathrm{Q}}_{\mathrm{I}}-\mathrm{x} \sigma_{\mathrm{y}_{\mathrm{e}}} \widetilde{\mathrm{w}}_{0}\right] / 2 \pi \\
& \overline{\mathrm{V}}_{\mathrm{pk}}=\hat{\mathrm{n}}_{\mathrm{k}}\left[\left(\mathrm{y}_{\mathrm{e}} \sigma_{\mathrm{y}_{\mathrm{e}}}-\sigma\right) \widetilde{\mathrm{w}}_{0}+\sigma_{\mathrm{z}_{\mathrm{e}}} \mathrm{R}_{\mathrm{s}}\right] / 2 \pi+\nabla \sigma\left(\mathrm{x} \widetilde{\mathrm{Q}}_{\mathrm{I}}-\mathrm{y}_{\mathrm{e}} \widetilde{\mathrm{w}}_{0}\right) / 2 \pi
\end{aligned}
$$

and $\hat{n}_{k}$ is the outward normal for the kth edge in the plane of the panel. 


\subsection{TRANSFORMATION OF VELOCITY COMPONENTS FROM LOCAL PANEL TO THE GLOBAL COORDINATE SYSTEM}

The influence coefficients for a given panel have been obtained in terms of local scaled panel coordinates. To find the contribution to the induced velocity on a given panel from all panels of the curved surface to be analyzed, we use a transformation from each panel coordinate system to the global system. For this purpose, we consider three basic coordinate systems:

1. Reference coordinates $\left(x_{r}, y_{r}, z_{r}\right)$ chosen for convenience in defining the body surface grid on which the panels are constructed.

2. Compressible coordinates $\left(x_{c}, y_{c}, z_{c}\right)$ in which the velocity of the free stream is aligned with the positive $x_{C}$ axis and, of course,

3. The local scaled panel coordinate system ( $\left.x_{p}, y_{p}, z_{p}\right)$

\subsection{TRANSFORMATION FROM THE REFERENCE COORDINATE SYSTEM TO THE COMPRESSIBLE SYSTEM}

It is frequently convenient on a configuration to use coordinates in which the $\mathrm{x}$ axis is not aligned in the free stream direction. We then must establish a transformation from this coordinate system to the system in which the compressibility scaling is applied. From the reference coordinate system $x_{r}, y_{r}, z_{r}$, we apply a rotation of the velocity vector through an angle of yaw $\beta_{C}$ followed by a rotation through an angle of attack $\alpha_{c}$ (see figure 23).

Here $\alpha_{\mathrm{c}}$ and $\beta_{\mathrm{c}}$ are not necessarily small. The first transformation in figure 23 is a rotation about the $\mathrm{y}_{\mathrm{r}}$ axis, Thus

$$
\begin{aligned}
& \mathrm{x}_{0}=\mathrm{x}_{\mathrm{r}} \cos \beta_{\mathrm{c}}+\mathrm{y}_{\mathrm{r}} \sin \beta_{\mathrm{c}} \\
& \mathrm{y}_{0}=-\mathrm{x}_{\mathrm{r}} \sin \beta_{\mathrm{c}}+\mathrm{y}_{\mathrm{r}} \cos \beta_{\mathrm{c}} \\
& \mathrm{z}_{0}=\mathrm{z}_{\mathrm{r}}
\end{aligned}
$$

We then apply a rotation $\alpha_{c}$ about the $y_{0}$ axis. This leads to the following transformation to compressible coordinates $\mathrm{x}_{\mathrm{c}}, \mathrm{y}_{\mathrm{c}}, \mathrm{z}_{\mathrm{c}}$ :

$$
\begin{aligned}
& \mathrm{x}_{\mathrm{c}}=\mathrm{x}_{0} \cos \alpha_{\mathrm{c}}-\mathrm{z}_{0} \sin \alpha_{\mathrm{c}} \\
& \mathrm{y}_{\mathrm{c}}=\mathrm{y}_{0} \\
& \mathrm{z}_{\mathrm{c}}=\mathrm{x}_{0} \sin \alpha_{\mathrm{c}}+\mathrm{z}_{0} \cos \alpha_{\mathrm{c}}
\end{aligned}
$$


Combining the results of the transformations yields

$$
\begin{aligned}
& \mathrm{x}_{\mathrm{c}}=\mathrm{x}_{\mathrm{r}} \cos \alpha_{\mathrm{c}} \cos \beta_{\mathrm{c}}+\mathrm{y}_{\mathrm{r}} \cos \alpha_{\mathrm{c}} \sin \beta_{\mathrm{c}}+\mathrm{z}_{\mathrm{r}} \sin \alpha_{\mathrm{c}} \\
& \mathrm{y}_{\mathrm{c}}=-\mathrm{x}_{\mathrm{r}} \sin \beta_{\mathrm{c}}+\mathrm{y}_{\mathrm{r}} \cos \beta_{\mathrm{c}} \\
& \mathrm{z}_{\mathrm{c}}=-\mathrm{x}_{\mathrm{r}} \sin \alpha_{\mathrm{c}} \cos \beta_{\mathrm{c}}-\mathrm{y}_{\mathrm{r}} \sin \alpha_{\mathrm{c}} \sin \beta_{\mathrm{c}}+\mathrm{z}_{\mathrm{r}} \cos \alpha_{\mathrm{c}}
\end{aligned}
$$

On defining the transformation matrix $\left\{A_{r}\right\}$ equation (10.3) takes the form

$$
\left(x_{c}, y_{c}, z_{c}\right)=\left\{A_{r}\right\}\left(\begin{array}{l}
x_{r} \\
y_{r} \\
z_{r}
\end{array}\right)
$$

where

$$
\left\{A_{r}\right\}=\left(\begin{array}{llc}
\cos \alpha_{c} \cos \beta_{c} & \cos \alpha_{c} \sin \beta_{c} & \sin \alpha_{c} \\
-\sin \beta_{c} & \cos \beta_{c} & 0 \\
-\sin \alpha_{c} \cos \beta_{c} & -\sin \alpha_{c} \sin \beta_{c} & \cos \alpha_{c}
\end{array}\right)
$$

When local panel coordinates $x_{p}, y_{p}, z_{p}$ are related to compressible coordinates by

$$
\left(x_{p}, y_{p}, z_{p}\right)=\left\{A_{c}\right\}\left(\begin{array}{l}
x_{c} \\
y_{c} \\
z_{c}
\end{array}\right)
$$

we have

$$
\left(x_{p}, y_{p}, z_{p}\right)=\left\{A_{c}\right\} \quad\left\{A_{r}\right\}\left(\begin{array}{l}
x_{r} \\
y_{r} \\
z_{r}
\end{array}\right)
$$


where $\left\{A_{c}\right\}$ is the matrix of transformation from the compressible coordinates to panel coordinates. Solving the set of equations (10.3) for $x_{r}, y_{T}, z_{T}$ in terms of $x_{c}, y_{c}, z_{c}$ yields

$$
\begin{aligned}
& \mathrm{x}_{\mathrm{r}}=\mathrm{x}_{\mathrm{c}} \cos \alpha_{\mathrm{c}} \cos \beta_{\mathrm{c}}-\mathrm{y}_{\mathrm{c}} \sin \beta_{\mathrm{c}}-\mathrm{z}_{\mathrm{c}} \sin \alpha_{\mathrm{c}} \cos \beta_{\mathrm{c}} \\
& \mathrm{y}_{\mathrm{r}}=\mathrm{x}_{\mathrm{c}} \cos \alpha_{\mathrm{c}} \sin \beta_{\mathrm{c}}+\mathrm{y}_{\mathrm{c}} \cos \beta_{\mathrm{c}}-\mathrm{z}_{\mathrm{c}} \sin \alpha_{\mathrm{c}} \sin \beta_{\mathrm{c}} \\
& \mathrm{z}_{\mathrm{r}}=\mathrm{x}_{\mathrm{c}} \sin \alpha_{\mathrm{c}}+\mathrm{z}_{\mathrm{c}} \cos \alpha_{\mathrm{c}}
\end{aligned}
$$

and we see that

$$
\left\{A_{r}{ }^{-1}\right\}=\left(\begin{array}{ccc}
\cos \alpha_{c} \cos \beta_{c} & -\sin \beta_{c} & -\sin \alpha_{c} \cos \beta_{c} \\
\cos \alpha_{c} \sin \beta_{c} & \cos \beta_{c} & -\sin \alpha_{c} \sin \beta_{c} \\
\sin \alpha_{c} & 0 & \cos \beta_{c}
\end{array}\right)
$$

and

$$
\left(x_{r}, y_{r}, z_{r}\right)=\left\{A_{r}{ }^{-1}\right\}\left(\begin{array}{c}
x_{c} \\
y_{c} \\
z_{c}
\end{array}\right)=\left\{A_{r}^{-1}\right\}\left\{A_{c}^{-1}\right\}\left(\begin{array}{c}
x_{p} \\
y_{p} \\
z_{p}
\end{array}\right)
$$

The normal in the compressibility coordinate system is defined in terms of the reference normal by

$$
\left\{A_{r}\right\} \hat{n}=\left(A^{(1)} \cdot \hat{n}, A_{r}{ }^{(2)} \cdot \hat{n}, A_{T}{ }^{(3)} \cdot \hat{n}\right)
$$

where $A_{r}(i)$ is the ith row of the matrix $\left\{A_{r}\right\}$. The conormal in the compressibility coordinate system is

$$
\left( \pm B^{2} A_{r}(1) \cdot \hat{n}, A_{r}^{(2)} \cdot \hat{n}, A_{r}(3) \cdot \hat{n}\right)
$$

where the plus sign holds for subsonic flow and minus sign for supersonic. 
If we define the matrix $\{B\}$ by

$$
\{B\}=\left(\begin{array}{ccc} 
\pm B^{2} & 0 & 0 \\
0 & 1 & 0 \\
0 & 0 & 1
\end{array}\right)
$$

then the conormal in the compressible system may be expressed as a matrix product

$$
\{B\}\left\{A_{r}\right\} \hat{n}
$$

In the reference coordinate system the conormal vector $\overline{\mathrm{n}}_{\mathrm{c}}$ is given by

$$
\bar{n}_{c} T=\left\{A_{r}{ }^{-1}\right\} \quad\{B\} \quad\left\{A_{r}\right\} \hat{n}
$$

where $\bar{n}_{c} T$ is a row vector.

\subsection{TRANSFORMATION FROM COMPRESSIBLE COORDINATES TO PANEL COORDINATE SYSTEM WITHOUT THE COMPRESSIBILITY FACTOR B IN THE PANEL SYSTEM}

For incompressible flow, the differential equation and distance are invariant under rotations about any axis, while for supersonic linearized theory, the differential equation and the hyperbolic distance are invariant under rotations only about the free stream direction ( $x_{c}$ axis) and under any oblique or Lorentz transformation in other planes. It is convenient to introduce at this point the transformation from scaled coordinates to compressible coordinates; namely

$$
\left(x_{s}, y_{s}, z_{s}\right)=\left(x_{c}, B y_{c}, B z_{c}\right)
$$

We now consider a rotation through an angle $\theta_{1}$ about $\mathrm{x}_{\mathrm{s}}$, the streamwise scaled compressible coordinate axis. This becomes

$$
\begin{aligned}
& \mathrm{x}_{1}=\mathrm{x}_{\mathrm{s}} \\
& \mathrm{y}_{1}=\mathrm{y}_{\mathrm{s}} \cos \theta_{1}-\mathrm{z}_{\mathrm{s}} \sin \theta_{1} \\
& \mathrm{z}_{1}=\mathrm{y}_{\mathrm{s}} \sin \theta_{1}+\mathrm{z}_{\mathrm{s}} \cos \theta_{1}
\end{aligned}
$$


Introducing compressible coordinates into equation (10.16) leads to

$$
\begin{aligned}
& \mathrm{x}_{1}=\mathrm{x}_{\mathrm{c}} \\
& \mathrm{y}_{1}=\mathrm{By}_{\mathrm{c}} \cos \theta_{1}-\mathrm{Bz}_{\mathrm{c}} \sin \theta_{1} \\
& \mathrm{z}=\mathrm{By}_{\mathrm{c}} \sin \theta_{1}+\mathrm{Bz}_{\mathrm{c}} \cos \theta_{1}
\end{aligned}
$$

We chose the angle $\theta_{1}$ so that the normal lies in the $y_{1}=0$ plane (see figure 24). Then

$$
\tan \theta_{1}=\mathrm{y}_{\mathrm{c}} / \mathrm{z}_{\mathrm{c}}=\mathrm{n}_{\mathrm{y}_{\mathrm{c}}} / \mathrm{n}_{\mathrm{z}_{\mathrm{c}}}
$$

from which $\sin \theta_{1}=n_{y_{c}} / \sqrt{n_{y_{c}}^{2}+n_{z_{c}}^{2}}$ and $\cos \theta_{1}=n_{z_{c}} / \sqrt{n_{y_{c}}{ }^{2}+n_{z_{c}}{ }^{2}}$.

The normal will be calculated generally in the reference coordinate system. Hence, we have, for the normal in the compressible system, from equation (10.10)

$$
n_{x_{c}}=A_{r}(1) \cdot \hat{n}, n_{y_{c}}=A_{r}(2) \cdot \hat{n}, n_{z_{c}}=A_{r}(3) \cdot \hat{n}
$$

where $A_{r}{ }^{(i)}$ denotes the ith row of the matrix $\left\{A_{r}\right\}$.

For subsonic flow, we rotate the $\mathrm{x}_{1}, \mathrm{y}_{1}, \mathrm{z}_{1}$ coordinates system about the $\mathrm{y}_{1}$ axis and the transformation becomes

$$
\begin{aligned}
& \mathrm{x}_{2}=\mathrm{x}_{1} \cos \theta_{2}-\mathrm{z}_{1} \sin \theta_{2} \\
& \mathrm{y}_{2}=\mathrm{y}_{1} \\
& \mathrm{z}_{2}=\mathrm{x}_{1} \sin \theta_{2}+\mathrm{z}_{1} \cos \theta_{2}
\end{aligned}
$$

We chose $\theta_{2}$ to make the panel lie in the $z_{2}=0$ plane; then from figure 25

$$
\tan \theta_{2}=-\mathrm{z}_{1} / \mathrm{x}_{1}=\mathrm{n}_{\mathrm{x}_{\mathrm{s}}} / \sqrt{\mathrm{n}_{\mathrm{y}_{\mathrm{s}}}^{2}+\mathrm{n}_{\mathrm{z}_{\mathrm{s}}}^{2}}
$$

from which

$$
\sin \theta_{2}=n_{x_{s}} \text { and } \cos \theta_{2}=\sqrt{n_{y_{s}}^{2}+n_{z_{s}}^{2}}
$$

Since the normal in the scaled coordinate system is related to the compressible system by

$$
n_{x_{s}}=B n_{x_{c}} / \sqrt{B^{2} n_{x_{c}}^{2}+n_{y_{c}}^{2}+n_{z_{c}}^{2}}
$$




$$
\begin{aligned}
& n_{y_{s}}=n_{y_{c}} / \sqrt{\mathrm{B}^{2} n_{x_{c}}{ }^{2}+n_{y_{c}}{ }^{2}+n_{z_{c}}{ }^{2}} \\
& n_{z_{s}}=n_{z_{c}} / \sqrt{\mathrm{B}^{2} n_{x_{c}}{ }^{2}+n_{y_{c}}{ }^{2}+n_{z_{c}}{ }^{2}}
\end{aligned}
$$

then

$$
\begin{aligned}
& \sin \theta_{2}=B n_{x_{c}} / \sqrt{\mathrm{B}^{2} n_{\mathrm{x}_{\mathrm{c}}}{ }^{2}+\mathrm{n}_{\mathrm{y}_{\mathrm{c}}}{ }^{2}+\mathrm{n}_{\mathrm{z}_{\mathrm{c}}}{ }^{2}} \\
& \cos \theta 2=\sqrt{n_{y_{c}}{ }^{2}+n_{z_{c}}{ }^{2}} / \sqrt{\mathrm{B}^{2} n_{x_{c}}{ }^{2}+n_{y_{c}}{ }^{2}+n_{z_{c}}{ }^{2}}
\end{aligned}
$$

Since the vector $\mathrm{B}^{2} \mathrm{n}_{\mathrm{x}_{\mathrm{c}}}, \mathrm{n}_{\mathrm{y}_{\mathrm{c}}}, \mathrm{n}_{\mathrm{z}_{\mathrm{c}}}$ is the conormal, we have

$$
\begin{gathered}
\sin \theta_{2}=\mathrm{Bn}_{\mathrm{x}_{\mathrm{c}}} \sqrt{\hat{\mathrm{n}} \cdot \overline{\mathrm{n}}_{\mathrm{c}}} \\
\cos \theta_{2}=\sqrt{\mathrm{n}_{\mathrm{y}_{\mathrm{c}}}^{2+\mathrm{n}_{\mathrm{y}_{\mathrm{c}}}{ }^{2}} / \sqrt{\hat{\mathrm{n}} \cdot \overline{\mathrm{n}}_{\mathrm{c}}}}
\end{gathered}
$$

For supersonic flow we apply an oblique transformation in the $x_{1}, z_{1}$ plane. This has the general form

$$
\begin{aligned}
& \mathrm{x}_{2}=\mathrm{x}_{1} / \sqrt{1-\mathrm{m}_{1}^{2}}+\mathrm{m}_{1} \mathrm{z}_{1} / \sqrt{1-\mathrm{m}_{1}^{2}} \\
& \mathrm{y}_{2}=\mathrm{y}_{1} \\
& \mathrm{z}_{2}=\mathrm{m}_{1} \mathrm{x}_{1} / \sqrt{1-\mathrm{m}_{1}^{2}}+\mathrm{z}_{1} / \sqrt{1-\mathrm{m}_{1}^{2}}
\end{aligned}
$$

For subinclined panels, we chose the coordinate $\mathrm{z}_{2}=0$ as the panel, then we obtain from figure 25

$$
\mathrm{m}_{1}=-\mathrm{z}_{1} / \mathrm{x}_{1}=\tan \theta_{2}
$$


Now

$$
\tan \theta_{2}=\frac{\mathrm{n}_{\mathrm{x}_{\mathrm{S}}}}{\sqrt{1-\mathrm{n}_{\mathrm{x}_{\mathrm{S}}^{2}}^{2}}}=\mathrm{m}_{1}
$$

Since

$$
\begin{aligned}
& \mathrm{n}_{\mathrm{x}_{\mathrm{s}}} \sim \mathrm{Bn}_{\mathrm{x}_{\mathrm{c}}} \\
& \mathrm{n}_{\mathrm{y}_{\mathrm{s}}} \sim \mathrm{n}_{\mathrm{y}_{\mathrm{c}}} \\
& \mathrm{n}_{\mathrm{z}_{\mathrm{s}}} \sim \mathrm{n}_{\mathrm{y}_{\mathrm{c}}}
\end{aligned}
$$

we have

$$
\frac{\mathrm{n}_{\mathrm{x}_{\mathrm{s}}}}{\sqrt{1-\mathrm{n}_{\mathrm{x}_{\mathrm{s}}}^{2}}}=\frac{\mathrm{n}_{\mathrm{x}_{\mathrm{s}}}}{\sqrt{\mathrm{n}_{\mathrm{y}_{\mathrm{s}}}^{2}+\mathrm{n}_{\mathrm{z}_{\mathrm{s}}^{2}}^{2}}}=\frac{\mathrm{Bn}_{\mathrm{x}_{\mathrm{c}}}}{\sqrt{\mathrm{n}_{\mathrm{y}_{\mathrm{c}}}^{2}+\mathrm{n}_{\mathrm{z}_{\mathrm{c}}}^{2}}}=\frac{\mathrm{Bn}_{\mathrm{x}_{\mathrm{c}}}}{\sqrt{1-\mathrm{n}_{\mathrm{x}_{\mathrm{c}}}^{2}}}=\mathrm{m}_{1}
$$

from which

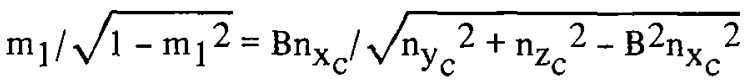

$$
\begin{aligned}
& =B n_{\mathrm{x}_{\mathrm{C}}} / \sqrt{\hat{\mathrm{n}} \cdot \overline{\mathrm{n}}_{\mathrm{C}}}
\end{aligned}
$$

and

$$
1 / \sqrt{1-m_{1}^{2}}=\sqrt{n_{y_{c}}{ }^{2}+n_{z_{c}}{ }^{2}} / \sqrt{\hat{n} \cdot \bar{n}_{c}}
$$

where $\bar{n}_{c}$ is the conormal given by

$$
\bar{n}_{c}=\left(-B n_{x_{c}}{ }^{2}, n_{y_{c}}, n_{z_{c}}\right)
$$

and

$$
\hat{n}=\left(n_{x_{c}}, n_{y_{c}}, n_{z_{c}}\right)
$$


Combining the two transformations yields the following tranformation from compressible coordinates, $x_{c}, y_{c}, z_{c}$ to $x_{p}, y_{p}, z_{p}=x_{2}, y_{2}, z_{2}$, the panel coordinates for supersonic flow:

$$
\begin{aligned}
& x_{p}=x_{c} / \sqrt{1-m_{1}^{2}}+m_{1} B\left(y_{c} \sin \theta_{1}+z_{c} \cos \theta_{1}\right) / \sqrt{1-m_{1}^{2}} \\
& y_{p}=B\left(y_{c} \cos \theta_{1}-z_{c} \sin \theta_{1}\right) \\
& z_{p}=m_{1} x_{c} / \sqrt{1-m_{1}^{2}}+B\left(y_{c} \sin \theta_{1}+z_{c} \cos \theta_{1}\right) / \sqrt{1-m_{1}^{2}}
\end{aligned}
$$

The determinant of the matrix of equation (10.31) is equal to $B^{2}$. Solving for $x_{c}, y_{c}, z_{c}$ in terms of $x_{p}, y_{p}, z_{p}$ yields

$$
\begin{aligned}
& \mathrm{x}_{\mathrm{C}}=\mathrm{x}_{\mathrm{p}} / \sqrt{1-\mathrm{m}_{1}^{2}}-\mathrm{m}_{1} \mathrm{z}_{\mathrm{p}} / \sqrt{1-\mathrm{m}_{1}^{2}} \\
& \mathrm{y}_{\mathrm{C}}=-\mathrm{x}_{\mathrm{p}} \mathrm{m}_{1} \sin \theta_{1} / \mathrm{B} \sqrt{1-\mathrm{m}_{1}^{2}}+\mathrm{y}_{\mathrm{p}} \cos \theta_{1} / \mathrm{B}+\mathrm{z}_{\mathrm{p}} \sin \theta_{1} / \mathrm{B} \sqrt{1-\mathrm{m}_{1}^{2}} \\
& \mathrm{z}_{\mathrm{C}}=-\mathrm{x}_{\mathrm{p}} \mathrm{m}_{1} \cos \theta_{1} / \mathrm{B} \sqrt{1-\mathrm{m}_{1}^{2}}-\mathrm{y}_{\mathrm{p}} \sin \theta_{1} / \mathrm{B}+\mathrm{z}_{\mathrm{p}} \cos \theta_{1} / \mathrm{B} \sqrt{1-\mathrm{m}_{1}^{2}}
\end{aligned}
$$

For superinclined panels we choose $\mathrm{m}_{1}$ so that $\mathrm{x}_{2}=0$ lies on the panel, then from equation (10.26) we get

$$
\begin{aligned}
& \mathrm{m}_{1}=-\mathrm{x}_{1} / \mathrm{z}_{1}=\cos \theta_{2} \\
& \mathrm{~m}_{1}=\sqrt{1-\mathrm{n}_{\mathrm{x}_{\mathrm{c}}}{ }^{2}} / \mathrm{Bn}_{\mathrm{x}_{\mathrm{c}}}=\sqrt{1-\left(\mathrm{A}_{\mathrm{r}}{ }^{(1) \cdot \hat{n})^{2}} / \mathrm{BA}_{\mathrm{r}}{ }^{(1)} \cdot \hat{\mathrm{n}}_{\mathrm{c}}\right.}
\end{aligned}
$$

from which

$$
m_{1} / \sqrt{1-m_{1}^{2}}=\sqrt{n_{y_{c}}^{2}+n_{z_{c}}^{2}} / \sqrt{-\hat{n} \cdot \bar{n}_{c}}
$$

and

$$
1 / \sqrt{1-m_{1}^{2}}=\mathrm{Bn}_{\mathrm{x}_{\mathrm{c}}} / \sqrt{-\hat{\mathrm{n}} \cdot \overline{\mathrm{n}}_{\mathrm{C}}}
$$

Since $\left|\mathrm{m}_{1}\right|<1$ we see that

$$
1 / \mathrm{B}<\mathrm{n}_{\mathrm{x}_{\mathrm{c}}} / \sqrt{1-\mathrm{n}_{\mathrm{x}_{\mathrm{C}}}^{2}}=\tan \theta_{2 \mathrm{c}}
$$


and the angle $\theta_{2 c}$ between the panel and free stream must be greater than the Mach angle. The matrix and its inverse have the same form for both superinclined and subinclined panels, but differ in the calculation of the parameter $\mathrm{m}_{1}$.

For the sake of convenience in the combined program for both subsonic and supersonic flow, we make the transformation for subsonic flow and for the subinclined and superinclined panels take the same form. Hence, we must rotate the coordinates of the superinclined panels so that $x_{p}, y_{p}$ are coordinates in the panel. Then the new panel coordinates are

$$
\begin{aligned}
& x_{p}=z_{2} \\
& y_{p}=-y_{2} \\
& z_{p}=x_{2}
\end{aligned}
$$

The variables $x_{2}, y_{2}, z_{2}$ correspond to $x, y, z$ in the derivation of the aerodynamic influence coefficients. Note that for superinclined panels the value of $m_{1}$ is the reciprocal of the value for subinclined panels. Let $\mathrm{m}_{2}$ be the value for superinclined panels; then substituting equation (10.34) and replacing $\mathrm{m}_{1}$ by $\mathrm{m}_{2}=1 / \mathrm{m}_{1}$ into equation (10.29) yields

$$
\begin{aligned}
& \mathrm{x}_{\mathrm{p}}=\mathrm{x}_{1} / \sqrt{\mathrm{m}_{1}^{2}-1}+\mathrm{m}_{1} \mathrm{z}_{1} / \sqrt{\mathrm{m}_{1}^{2}-1} \\
& \mathrm{y}_{\mathrm{p}}=-\mathrm{y}_{1}=\mathrm{y}_{1} \operatorname{sign}\left(1-\mathrm{m}_{1} 2\right) \\
& \mathrm{z}_{\mathrm{p}}=\mathrm{m}_{1} \mathrm{x}_{1} / \sqrt{\mathrm{m}_{1}^{2}-1}+\mathrm{z}_{1} / \sqrt{\mathrm{m}_{1}^{2}-1}
\end{aligned}
$$

with $m_{1}$ defined by equation (10.30). When $m_{1}^{2}-1$ is replaced by $\left|1-m_{1}^{2}\right|$ then equation (10.35) holds for both subinclined and superinclined panels. Since for subsonic flow

$$
\mathrm{B}^{2} \mathrm{n}_{\mathrm{x}_{\mathrm{c}}}{ }^{2}+\mathrm{n}_{\mathrm{y}_{\mathrm{c}}}{ }^{2}+\mathrm{n}_{\mathrm{z}_{\mathrm{c}}}{ }^{2}=\hat{\mathrm{n}} \cdot \overrightarrow{\mathrm{n}}_{\mathrm{c}}
$$

we find that the transformation from the reference coordinate system to the panel coordinate system in both subsonic and supersonic flows can be written in the form

$$
\left(x_{p}, y_{p}, z_{p}\right)=\left\{A_{2}\right\} \quad\left\{A_{1}\right\} \quad\left\{A_{r}\right\}\left(\begin{array}{l}
x_{r}-x_{0} \\
y_{r}-y_{0} \\
z_{r}-z_{0}
\end{array}\right)
$$


where $\left\{A_{r}\right\}$ is given in equation $(10.5), x_{0}, y_{0}, z_{0}$ is the center of the panel and the origin of the panel coordinate system,

$$
\left\{\mathrm{A}_{1}\right\}=\left(\begin{array}{ccc}
1 & 0 & 0 \\
0 & \mathrm{Bn}_{\mathrm{z}_{\mathrm{c}}} / \gamma & -\mathrm{Bn}_{\mathrm{y}_{\mathrm{c}}} / \gamma \\
0 & \mathrm{Bn}_{\mathrm{y}_{\mathrm{c}}} / \gamma & \mathrm{Bn}_{\mathrm{z}_{\mathrm{c}}} / \gamma
\end{array}\right)
$$

and

$$
\left\{\mathrm{A}_{2}\right\}=\left(\begin{array}{ccc}
\gamma / \sqrt{\left|\hat{\mathrm{n}} \cdot \overline{\mathrm{n}}_{\mathrm{c}}\right|} & 0 & -\mathrm{sBn_{x_{c } }} / \sqrt{\left|\hat{\mathrm{n}} \cdot \overline{\mathrm{n}}_{\mathrm{c}}\right|} \\
0 & \sin \left(\hat{\mathrm{n}} \cdot \overline{\mathrm{n}}_{\mathrm{c}}\right) & 0 \\
\mathrm{Bn}_{\mathrm{x}_{\mathrm{c}}} / \sqrt{\left|\hat{\mathrm{n}} \cdot \overline{\mathrm{n}}_{\mathrm{c}}\right|} & 0 & \gamma / \sqrt{\left|\hat{\mathrm{n}} \cdot \overline{\mathrm{n}}_{\mathrm{c}}\right|}
\end{array}\right)
$$

where $s=-1$ if the flow is supersonic and $s=1$ if the flow is subsonic;

$$
\gamma=\sqrt{\mathrm{n}^{2} \mathrm{y}_{\mathrm{c}}+\mathrm{n}^{2} \mathrm{z}_{\mathrm{c}}} \quad \text { and } \quad \mathrm{B}=\sqrt{\left|1-\mathrm{M}^{2}\right|}
$$

The points defining the panel are required in the average plane panel coordinates. Similarly the corner points defining the triangular subpanels are also required in the local subpanel coordinate system. All these coordinates are computed using equation (10.37) as defined for the particular pancl or subpancl. The projection of the curved pancl on the average plane is found by setting $z_{p}=0$.

For supersonic flow, the quantity $\hat{n} \cdot \bar{n}_{C}$ can be seen to be a test for sub- or superinclined panels. If $\hat{n} \cdot \bar{n}_{c}>0$, the panel is subinclined, if $\hat{n} \cdot \vec{n}_{c}=0$, the panel is inclined at the Mach angle; and if $\hat{n} \cdot \bar{n}_{\mathrm{c}}<0$, the panel is superinclined. Panels inclined at the Macli angle are not admissible since the transformation to compressible coordinates breaks down. This can be seen by setting $m_{1}=1$ in equation (10.26).

\subsection{CORRECTION TO PANEL ELEMENT OF AREA IN SUPERSONIC FLOW DUE TO THE TRANSFORMATION FROM GLOBAL, TO PANEL COORDINATES}

The influence coefficients are computed in the coordinate system of the panel inducing the flow. Hence the conormal derivative in the compressible direction and the element of area ds must be transformed. The factor from page 383 of Courant [16], Vol. 2, relating the surface panel element of area $d x_{p} d y_{p}$ to $d s$ is

$$
d s=\left[n_{x_{c}} \frac{\partial\left(y_{c}, z_{c}\right)}{\partial\left(x_{p}, y_{p}\right)}+n_{y_{c}} \frac{\partial\left(z_{c}, x_{c}\right)}{\partial\left(x_{p}, y_{p}\right)}+n_{z_{c}} \frac{\partial\left(x_{c}, y_{c}\right)}{\partial\left(x_{p}, y_{p}\right)}\right] d x_{p} d y_{p}
$$


Evaluating the Jacobians from equation (10.32) yields

$$
\mathrm{ds}=\left[\mathrm{n}_{\mathrm{x}_{\mathrm{c}}} \mathrm{m}_{1} / \mathrm{B}+\left(\mathrm{n}_{\mathrm{y}_{\mathrm{c}}} \sin \theta_{1}+\mathrm{n}_{\mathrm{z}_{\mathrm{c}}} \cos \theta_{1}\right)\right] \mathrm{dx_{ \textrm {p } }} d \mathrm{y}_{\mathrm{p}} / \mathrm{B} \sqrt{1-\mathrm{m}_{1}^{2}}
$$

Substituting for $m_{1}$ from equation (10.30) and $\theta_{1}$ from equation (10.18) finally gives us

$$
d s=d x_{p} d y_{p} / B \sqrt{1-M^{2} n_{x_{c}}^{2}}
$$

for subinclined panels. For superinclined panels the area element on the panel is in the $y_{p}$, $\mathrm{z}_{\mathrm{p}}$ coordinates and surface elements are related by

$$
d_{s}=\left[n_{x_{c}} \frac{\partial\left(y_{c}, z_{c}\right)}{\partial\left(y_{p}, z_{p}\right)}+n_{y_{c}} \frac{\partial\left(z_{c}, x_{c}\right)}{\partial\left(y_{p}, z_{p}\right)}+n_{z_{c}} \frac{\partial\left(x_{c}, y_{c}\right)}{\partial\left(y_{p}, z_{p}\right)}\right] d y_{p} d z_{p}
$$

Here we used the transformation in equations (10.31) and (10.32) instead of equation (10.34) through (10.39). Evaluating the Jacobians using equation (10.32) yields

$$
\mathrm{ds}=\left[\mathrm{n}_{\mathrm{x}_{\mathrm{c}}} / \mathrm{B}+\mathrm{m}_{1}\left(\mathrm{n}_{\mathrm{y}_{\mathrm{c}}} \sin \theta_{1}+\mathrm{n}_{\mathrm{z}_{\mathrm{c}}} \cos \theta_{1}\right)\right] d \mathrm{y}_{\mathrm{p}} \mathrm{dz}_{\mathrm{p}} / \mathrm{B} \sqrt{1-\mathrm{m}_{1}^{2}}
$$

Finally, substituting equation (10.33) for $\mathrm{m}_{1}$ and equation (10.18) for $\theta_{1}$ gives us

$$
d s=d y_{p} d z_{p} / B \sqrt{M^{2} n_{x_{c}}{ }^{2}-1}
$$

for superinclined panels.

The integrals involving the doublet do not need the area factor if the conormal derivative is replaced by the derivative in the panel coordinate $z_{p}$ for subinclined panels and $x_{p}$ for superinclined panels. For subinclined panels, the conormal derivative takes the form

$$
\begin{aligned}
\partial \phi / \partial \mathrm{n}_{\mathrm{c}}= & -\mathrm{B}^{2} \mathrm{n}_{\mathrm{x}_{\mathrm{c}}} \partial \phi / \partial \mathrm{x}_{\mathrm{c}}+\mathrm{n}_{\mathrm{y}_{\mathrm{c}}} \partial \phi / \partial \mathrm{y}_{\mathrm{c}}+\mathrm{n}_{\mathrm{z}_{\mathrm{c}}} \partial \phi / \partial \mathrm{z}_{\mathrm{c}} \\
\partial \phi / \partial \mathrm{n}_{\mathrm{c}}= & \left(-\mathrm{B}^{2} \mathrm{n}_{\mathrm{x}_{\mathrm{c}}} \partial \mathrm{x}_{\mathrm{p}} / \partial \mathrm{x}_{\mathrm{c}}+\mathrm{n}_{\mathrm{y}_{\mathrm{c}}} \partial \mathrm{x}_{\mathrm{p}} / \partial \mathrm{y}_{\mathrm{c}}+\mathrm{n}_{\mathrm{z}_{\mathrm{c}}} \partial \mathrm{x}_{\mathrm{p}} / \partial \mathrm{z}_{\mathrm{c}}\right) \partial \phi / \partial \mathrm{x}_{\mathrm{p}} \\
& +\left(-\mathrm{B}^{2} \mathrm{n}_{\mathrm{x}_{\mathrm{c}}} \partial \mathrm{y}_{\mathrm{p}} / \partial \mathrm{x}_{\mathrm{c}}+\mathrm{n}_{\mathrm{y}_{\mathrm{c}}} \partial \mathrm{y}_{\mathrm{p}} / \partial \mathrm{y}_{\mathrm{c}}+\mathrm{n}_{\mathrm{z}_{\mathrm{c}}} \partial \mathrm{y}_{\mathrm{p}} / \partial \mathrm{z}_{\mathrm{c}}\right) \partial \phi / \partial \mathrm{y}_{\mathrm{p}} \\
& +\left(-\mathrm{B}^{2} \mathrm{n}_{\mathrm{x}_{\mathrm{c}}} \partial \mathrm{z}_{\mathrm{p}} / \partial \mathrm{x}_{\mathrm{c}}+\mathrm{n}_{\mathrm{y}_{\mathrm{c}}} \partial \mathrm{z}_{\mathrm{p}} / \partial \mathrm{y}_{\mathrm{c}}+\mathrm{n}_{\mathrm{z}_{\mathrm{c}}} \partial \mathrm{z}_{\mathrm{p}} / \partial \mathrm{z}_{\mathrm{c}}\right) \partial \phi / \partial \mathrm{z}_{\mathrm{p}}
\end{aligned}
$$


The coefficients of $\frac{\partial \phi}{\partial x_{p}}$ and $\frac{\partial \phi}{\partial y_{p}}$ are found to equal zero when the derivatives inside the parentheses are evaluated from equation (10.31) with $\theta_{1}$ and $\mathrm{m}_{1}$ determined by equations (10.18) and (10.30), respectively. Evaluating the coefficient of $\partial \phi / \partial z_{p}$ yields

$$
\frac{\partial \phi}{\partial n_{c}}=-B \sqrt{M^{2} n_{x_{c}}^{2}-1} \frac{\partial \phi}{\partial x_{p}}
$$

and we see that

$$
\frac{\partial \phi}{\partial n_{c}} d s=\frac{\partial \phi}{\partial z_{p}} d x_{p} d y_{p}
$$

When the same procedure is applied to the superinclined panels we obtain

$$
\frac{\partial \phi}{\partial \mathrm{n}_{\mathrm{C}}}=\mathrm{B} \sqrt{1-\mathrm{M}^{2} \mathrm{n}_{\mathrm{x}_{\mathrm{c}}}^{2}} \frac{\partial \phi}{\partial \mathrm{z}_{\mathrm{p}}}
$$

and

$$
\frac{\partial \phi}{\partial n_{c}} d s=-\frac{\partial \phi}{\partial x_{p}} d y_{p} d z_{p}
$$

Hence the area correction term need not be applied to the doublet integrals.

\subsection{TRANSFORMATION OF VELOCITY COMPONENTS TO COMPONENTS IN THE REFERENCE SYSTEM}

The velocity components are computed in the coordinate system of the panel inducing the flow. In terms of the compressibility direction, the velocity components are found from the chain rule; i.e.,

$$
\frac{\partial \phi}{\partial x_{c}}=\frac{\partial \phi}{\partial x_{p}} \frac{\partial x_{p}}{\partial x_{c}}+\frac{\partial \phi}{\partial y_{p}} \frac{\partial y_{p}}{\partial x_{c}}+\frac{\partial \phi}{\partial z_{p}} \frac{\partial z_{p}}{\partial x_{c}}
$$

with similar relations for $\frac{\partial \phi}{\partial \mathrm{y}_{\mathrm{c}}}$ and $\frac{\partial \phi}{\partial \mathrm{z}_{\mathrm{c}}}$. Using equation (10.31) to evaluate the coefficients of $\frac{\partial \phi}{\partial x_{p}}, \frac{\partial \phi}{\partial y_{p}}$, and $\frac{\partial \phi}{\partial z_{p}}$ yields 


$$
\begin{aligned}
& \frac{\partial \phi}{\partial \mathrm{x}_{\mathrm{c}}}=\frac{\partial \phi}{\partial \mathrm{x}_{\mathrm{r}}} \frac{1}{\sqrt{1-\mathrm{m}_{1}^{2}}}+\frac{\partial \phi}{\partial \mathrm{z}_{\mathrm{p}}} \frac{\mathrm{m}_{1}}{\sqrt{1-\mathrm{m}_{1}^{2}}} \\
& \frac{\partial \phi}{\partial \mathrm{y}_{\mathrm{c}}}=\frac{\partial \phi}{\partial \mathrm{x}_{\mathrm{p}}} \frac{\mathrm{m} \mathrm{B} \sin \theta}{\sqrt{1-\mathrm{m}_{1}^{2}}}+\frac{\partial \phi}{\partial \mathrm{y}_{\mathrm{p}}} \mathrm{B} \cos \theta_{1}+\frac{\partial \phi}{\partial \mathrm{z}_{\mathrm{p}}} \frac{\mathrm{B} \sin \theta}{\sqrt{1-\mathrm{m}_{1}^{2}}} \\
& \frac{\partial \phi}{\partial \mathrm{z}_{\mathrm{c}}}=\frac{\partial \phi}{\partial \mathrm{x}_{\mathrm{p}}} \frac{\mathrm{m}_{1} \mathrm{~B} \cos \theta_{1}}{\sqrt{1-\mathrm{m}^{2}}}-\frac{\partial \phi}{\partial \mathrm{y}_{\mathrm{c}}} \mathrm{B} \sin \theta_{1}+\frac{\partial \phi}{\partial \mathrm{z}_{\mathrm{p}}} \frac{\mathrm{B} \cos \theta_{1}}{\sqrt{1-\mathrm{m}_{1}^{2}}}
\end{aligned}
$$

We see that this may be expressed in the form

$$
\overline{\mathrm{V}}_{\mathrm{c}}=\left\{\mathrm{A}^{\mathrm{T}}\right\} \overline{\mathrm{V}}_{\mathrm{p}}
$$

where $\left\{\mathrm{A}^{\mathrm{T}}\right\}$ is the transpose of $\{\mathrm{A}\}$, the matrix of equation (10.31).

We now resolve the vector $\bar{V}_{c}$ into components along the coordinate axes of the reference coordinate system. Thus

$$
\vec{V}=\left\{A_{r}^{-1}\right\} \bar{V}_{c}=\left\{A_{r}^{T}\right\} \vec{V}_{c}=\left\{A_{r}^{T}\right\}\left\{A^{T}\right\} \vec{V}_{p}
$$

or

$$
\bar{V}=\left(\{A\}\left\{A_{r}\right\}\right)^{T} \bar{V}_{p}
$$

This formula is valid for both subsonic and supersonic flow. 


\subsection{FAR FIELD EXPANSIONS FOR THE AERODYNAMIC INFLUENCE COEFFICIENTS}

\subsection{SOURCE AERODYNAMIC INFLUENCE COEFFICIENTS}

For panels lying wholly within and sufficiently far from the Mach cone emanating upstream of the control point, far field expansions of the aerodynamic influence coefficients are feasible. These expansions are algebraic and are much more economical in computing time. Except for a few signs, the subsonic and supersonic far field expansions are identical in form and a single derivation of the far field aerodynamic influence coefficients is possible.

It is convenient to represent the far field integrals in the compressible coordinate system rather than in the panel system. We shall translate the origin of coordinates to the center of the panel inducing the flow to simplify the analysis. Let $\mathrm{P}$ denote the control point and $\mathrm{Q}$ be the location of the singularity point on the panel and the coordinates of integration. The distance, or hyperbolic distance, is given by the scalar product

$$
R_{B}=[\bar{P}-\bar{Q}, \bar{P}-\bar{Q}]^{1 / 2}
$$

where the scalar product $[\bar{A}, \bar{B}]$ is defined by

$$
[\bar{A}, \bar{B}]=a_{i} c_{i j} b_{j}
$$

and $c_{i j}$ is the matrix

$$
\{C\}=c_{i j}=\left(\begin{array}{ccc}
1 & 0 & 0 \\
0 & s B^{2} & 0 \\
0 & 0 & s B^{2}
\end{array}\right)
$$

and $B=\sqrt{\left|1-M^{2}\right|}, s=1$ for subsonic flow and -1 for supersonic.

To utilize subscript notation effectively, we let

$$
\overline{\mathrm{P}}=\left(\mathrm{x}_{1}, \mathrm{x}_{2}, \mathrm{x}_{3}\right)
$$

and

$$
\overline{\mathrm{Q}}=\left(\mathrm{y}_{1}, \mathrm{y}_{2}, \mathrm{y}_{3}\right)
$$


The expansion of $1 / R_{B}$ then becomes

$$
\begin{aligned}
& \frac{1}{R_{B}}= \frac{1}{R_{c}}-y_{i} \frac{\partial}{\partial x_{i}}\left(\frac{1}{R_{c}}\right)+\frac{1}{2} y_{i} y_{j} \frac{\partial^{2}}{\partial x_{i} \partial x_{j}}\left(\frac{1}{R_{c}}\right)-\frac{1}{6} y_{i} y_{j} y_{k} \frac{\partial^{3}}{\partial x_{i} \partial x_{j} \partial x_{k}}\left(\frac{1}{R_{c}}\right) \\
& \frac{1}{R_{B}}=\frac{1}{R_{c}}+\frac{y_{i} c_{i j} x_{j}}{R_{c}^{3}}+\frac{1}{2} y_{j} y_{j}\left(-\frac{c_{i j}}{R_{c}^{3}}+\frac{3 \tilde{x}_{i} \tilde{x}_{j}}{R_{c}{ }^{3}}\right) \\
&-\frac{1}{6} y_{i} y_{j} y_{k}\left[\left(3 / R_{c} 5\right)\left(c_{i k} \tilde{x}_{j}+c_{j k} \tilde{x}_{i}+c_{i j} \tilde{x}_{k}\right)-15 \tilde{x}_{i} x_{j} x_{k} / R_{c}{ }^{7}\right]
\end{aligned}
$$

where $R_{c}=[\bar{P}, \bar{P}]^{J / 2}$ is the distance for subsonic flow, or hyperbolic distance for supersonic flow, from the panel center to the control point and $\tilde{x}_{i}=x_{j} c_{i j}$. Using the fact that $\mathrm{y}_{\mathrm{i}} \tilde{\mathrm{x}}_{\mathrm{i}}=\tilde{\mathrm{y}}_{\mathrm{i}} \mathrm{x}_{\mathrm{i}}$, we obtain

$$
\frac{1}{R_{B}}=G^{(0)}+\tilde{y}_{i} G_{i}^{(1)}+\frac{1}{2} \tilde{y}_{i} \tilde{y}_{j} G_{i j}(2)+\frac{1}{6} \tilde{y}_{i} \tilde{y}_{j} \tilde{y}_{k} G_{i j k}^{(4)}
$$

where

$$
\begin{aligned}
G^{(0)} & =1 / R_{c} \\
G_{i} & =x_{i} / R_{c}^{3} \\
G_{i j}(2) & =3 x_{i} x_{j} / R_{c}^{5}-c_{i j}-1 / R_{c}^{3} \\
G_{i j k}(4) & =15 x_{i} x_{j} x_{k} / R_{c}^{7}-\left(3 / R_{c}^{5}\right)\left(c_{i k}-1 x_{j}+c_{j k}-1 x_{i}+c_{i j}-1 x_{k}\right)
\end{aligned}
$$

To compute the velocity vector we obtain

$$
\begin{gathered}
\left\{-\nabla_{p}\left(1 / R_{B}\right)\right\}_{k}=-\left[\frac{\partial}{\partial x_{k}}\left(\frac{1}{R_{c}}\right)-y_{i} \frac{\partial}{\partial x_{i}} \partial x_{k}\left(\frac{1}{R_{c}}\right)+\frac{1}{2} y_{i} y_{j} \frac{\partial^{3}}{\partial x_{i} \partial x_{j} \partial x_{k}}\left(\frac{1}{R_{c}}\right)\right]+\ldots \\
\left\{-C^{-1} \nabla_{p}\left(1 / R_{B}\right)\right\}_{k}=G_{k}(1)+\tilde{y}_{i} G_{i k}(2)+\frac{1}{2} \tilde{y}_{i} \tilde{y}_{j} G_{i j k}(4)+\ldots
\end{gathered}
$$


The source potential is given by substituting equation (11.5) into

$$
\phi=-(1 / \omega) \int \sigma \mathrm{ds} / \mathrm{R}_{\mathrm{B}}
$$

where $\omega=2 \pi$ for supersonic flow and $4 \pi$ for subsonic. We obtain

$$
\phi_{s}=-G^{(0)} E^{(1)}-G_{i}^{(1)} E_{i}(2)-G_{i j}(2) E_{i j}(4)
$$

where

$$
\begin{aligned}
& \mathrm{E}^{(1)}=(1 / \omega) \int \sigma \mathrm{ds} \\
& \mathrm{E}_{\mathbf{i}}^{(2)}=(1 / \omega) \int \sigma \tilde{\mathrm{y}}_{\mathbf{i}} \mathrm{ds} \\
& \mathrm{E}_{\mathrm{ij}}^{(4)}=(1 / \omega) \int \sigma \tilde{y}_{\mathbf{i}} \tilde{\mathrm{y}}_{\mathbf{j}} \mathrm{ds}
\end{aligned}
$$

Similarly, the source velocity components are given by

$$
\begin{aligned}
& \overline{\mathrm{V}}_{\mathrm{s}}=-\frac{1}{\omega} \int \sigma \nabla_{\mathrm{p}}\left(1 / \mathrm{R}_{\mathrm{B}}\right) \mathrm{ds} \\
& \left\{C^{-1}\right\} \bar{V}_{s}=\frac{1}{\omega} \int \sigma\left[-\left\{C^{-1}\right\} \nabla_{p}\left(1 / R_{B}\right)\right] d s \\
& \left\{c^{-1}\right\} \bar{V}_{s}=G_{k}(1) E(1)+G_{i k}(2) E_{i}(2)+G_{i j k}(4) E_{i j}(4)
\end{aligned}
$$

The integration of the $\mathrm{E}$ integrals are performed in panel coordinates. The transformation of the $y$ variables to panel coordinates is affected by

$$
\widetilde{Y}=\{c\} Y=\{C\}\left\{A^{-1}\right\}(\xi, \eta, 0)
$$

and the element of area is

$$
\mathrm{ds}=\mathrm{J} \mathrm{d} \xi \mathrm{d} \eta
$$


where $\mathrm{J}$ is the Jacobian of the transformation from compressible to panel coordinates whose matrix is A. Since $\sigma=\sigma_{\mathrm{O}}+\sigma_{\mathrm{x}} \xi+\sigma_{\mathrm{y}} \eta$, the Matrices $\mathrm{E}$ are products of the matrices relating $\sigma_{\mathrm{O}}, \sigma_{\mathrm{X}}, \sigma_{\mathrm{y}}$ to the values of the source at panel centers of neighboring panels, of the matrices \{C\}, $\left\{\mathrm{A}^{-1}\right\}$, and the moments

$$
\mathrm{C}_{\mathrm{mn}}=\iint \xi^{\mathrm{m}+1} \eta^{\mathrm{n}+1} \mathrm{~d} \xi \mathrm{d} \eta
$$

where integration is over the panel. The development of the formulae is fairly straight forward but tedious and is omitted.

\subsection{DOUBLET AERODYNAMIC INFLUENCE COEFFICIENTS}

The doublet potential is given by

$$
\begin{aligned}
\phi & =\frac{1}{\omega} \int \mu \overline{\mathrm{n}}_{\mathrm{c}} \cdot \nabla_{\mathrm{p}}\left(1 / \mathrm{R}_{\mathrm{B}}\right) \mathrm{ds} \\
& -\frac{1}{\omega} \int \mu \overline{\mathrm{n}}_{\mathrm{c}}\{\mathrm{C}\}\left[-\left\{\mathrm{C}^{-1}\right\} \nabla_{\mathrm{p}}\left(1 / \mathrm{R}_{\mathrm{B}}\right)\right] \mathrm{ds}
\end{aligned}
$$

Since $\bar{n}_{c}=\hat{n}\{B\}$, then $\bar{n}_{c}\{C\}=\hat{n}^{T}\{B\} \quad\{C\}=s^{2} \hat{n}^{T}$. Here

$$
\{B\}=\left(\begin{array}{ccc}
\mathrm{sB}^{2} & 0 & 0 \\
0 & 1 & 0 \\
0 & 0 & 1
\end{array}\right)
$$

and $\hat{n}^{\mathrm{T}}$ is a column vector.

then

$$
\phi=-G_{k}(1) W_{k}(1)-G_{k i}{ }^{(2)} W_{k i}(2)-G_{k i j}{ }^{(4)} W_{k i j}(4)
$$

where

$$
\begin{aligned}
& \mathrm{W}_{\mathrm{k}}(1)=\left(\mathrm{sB}^{2} / \omega\right) \int \mu \mathrm{n}_{\mathrm{k}} \mathrm{ds} \\
& \mathrm{w}_{\mathrm{ki}}(2)=\left(\mathrm{sB}^{2} / \omega\right) \int \mu \mathrm{n}_{\mathrm{k}} \tilde{\mathrm{y}}_{\mathrm{i}} \mathrm{ds} \\
& \mathrm{w}_{\mathrm{kij}}(4)=\left(\mathrm{sB}^{2} / \omega\right) \int \mu \mathrm{n}_{\mathrm{k}} \tilde{\mathrm{y}}_{\mathrm{i}} \tilde{\mathrm{y}}_{\mathrm{j}} \mathrm{ds}
\end{aligned}
$$


The evaluation of the $\mathrm{W}$ matrices is carried out in a manner similar to the $\mathrm{E}$ matrices.

The velocity components for the far field are evaluated with the line integrals of the doublet strength neglected. One can easily verify that in the second term of equation (3.51)

$$
\begin{aligned}
\mathrm{B}^{2}\left(\overline{\mathrm{R}}_{0}-\overline{\mathrm{R}}\right) / \mathrm{R}_{\mathrm{B}}{ }^{3} & =-\{\mathrm{B}\} \nabla_{\mathrm{p}}\left(1 / \mathrm{R}_{\mathrm{B}}\right) \\
& =-\{\mathrm{B}\}\{\mathrm{C}\}\left[-\left\{\mathrm{C}^{-1}\right\} \nabla_{\mathrm{p}}\left(1 / \mathrm{R}_{\mathrm{B}}\right)\right] \\
& =\mathrm{sB}^{2}\left[-\left\{\mathrm{C}^{-1}\right\} \nabla_{\mathrm{p}}\left(1 / \mathrm{R}_{\mathrm{B}}\right)\right]
\end{aligned}
$$

Substituting the equation (11.14) into the second term of equation (3.51) yields, with the aid of equation (11.7),

$$
\begin{aligned}
\bar{V}_{\mathrm{d}} & =\frac{\mathrm{sB}}{\omega} \int(\hat{\mathrm{n}} \times \nabla \mu) \times\left[-\left\{\mathrm{C}^{-1}\right\} \nabla_{\mathrm{p}}\left(1 / \mathrm{R}_{\mathrm{B}}\right)\right] \mathrm{ds} \\
& =\frac{\mathrm{sB}}{\omega} \int(\hat{\mathrm{n}} \times \nabla \mu) \times\left[\mathrm{G}^{(1)}+\tilde{\mathrm{y}}_{\mathrm{i}} \mathrm{G}_{\mathrm{i}}^{(2)}+\frac{1}{2} \tilde{y}_{\mathrm{i}} \tilde{\mathrm{y}}_{\mathrm{j}} \mathrm{G}_{\mathrm{ij}}(4)\right] \mathrm{ds} \\
\bar{V}_{\mathrm{d}} & =\mathrm{G}^{(1)} \times \overline{\mathrm{F}}^{(1)}+\mathrm{G}_{\mathrm{i}}(2) \times \overline{\mathrm{F}}_{\mathrm{i}}(2)+\mathrm{G}_{\mathrm{ij}}(4) \times \overline{\mathrm{F}}_{\mathrm{ij}}(4)
\end{aligned}
$$

The sign of equation (3.51) has been changed since we are using a normal $\hat{n}$ into the fluid. Here

$$
\begin{aligned}
& \overline{\mathrm{F}}(1)=\left(\mathrm{sB}^{2} / \omega\right) \int(\hat{\mathrm{n}} \times \nabla \mu) \mathrm{ds} \\
& \overline{\mathrm{F}}_{\mathrm{i}}(2)=\left(\mathrm{sB}^{2} / \omega\right) \int(\hat{\mathrm{n}} \times \nabla \mu) \mathrm{y}_{\mathrm{i}} \mathrm{ds} \\
& \overline{\mathrm{F}}_{\mathrm{ij}}(4)=\left(\mathrm{sB}^{2} / \omega\right) \int(\hat{\mathrm{n}} \times \nabla \mu) \tilde{y}_{\mathrm{i}} \tilde{\mathrm{y}}_{\mathrm{j}} \mathrm{d} \mathrm{s}
\end{aligned}
$$

Again, the integration is over the panel. The $\mathrm{F}$ matrices are again products of several matrices, relating transformation to panel coordinates, relating the coefficients of $\mu$ to surrounding center panel values of doublet strength, and of the moment integrals $C_{m n}$ in Equation (11.11). 


\subsection{DISCUSSION OF RESULTS}

\subsection{SOURCE PANEL METHOD APPLIED TO CONES AND BODIES OF REVOLUTION}

The source panel method was first applied to cones and slender bodies of revolution. For a free stream Mach number of $\sqrt{2}$, figure 26 shows the pressure coefficient versus cone half-angle for zero angle of attack. The pressure coefficient agrees well with the exact solution and begins to depart from the theory only for cone half-angles approaching the shock detachment angle. Figure 27 gives a comparison of the exact solution from references [17] through [20] for the flow over $10^{\circ}$ and $15^{\circ}$ half-angle cones over a range of Mach numbers with the present method, and with the method modified to apply tangential velocity boundary conditions instead of tangential mass flux boundary conditions. Solutions with tangential velocity boundary conditions generally underpredict the pressure magnitude. The agreement with exact theory is very good for the $10^{\circ}$ cone up to a free stream Mach number of 3 . For the $15^{\circ}$ cone, agreement with exact theory is excellent up to a free stream Mach number of 1.6 , but drops rapidly for higher Mach numbers. The rapid drop in pressure occurs for ncar stagnation regions of the flow when mass flux boundary conditions are used. A modification of the present calculation to give better results in stagnation flow is presented in the section 12.2 on elliptic cones.

For a $10^{\circ}$ half-angle cone at $5^{\circ}$ angle of attack the source panel method with tangential mass flux boundary conditions using 36 panels around $180^{\circ}$ of the cone axis is compared with the solution from tangential velocity boundary condilions and with exact theory of references [17] to [20] in figure 28. The velocity boundary condition underpredicts the pressure especially on the high pressure side of the cone.

Comparison of the pressure coefficient on an axially symmetric spindle computed by the linear source panel method with results from the exact method of characteristics and with a line source distribution solution is presented in figure 29 and the three methods are in good agreement. Here mass flux and tangential velocity boundary conditions would be expected to be in closer agreement, since the body slopes are small. Also shown on the graph are results from a constant source panel method using the same panel distribution. The present method with linearly varying source distributions is seen to yield better results.

To test the stability of the source numerical method a spindle was paneled in a random manner as shown in figure 30 . The resulting values of the pressure distribution at the control points of the panel is indicated by the dots in figure 31 . Considering the extreme variation in panel size, we see that the agreement with the exact calculations is very good and that the numcrical method is remarkably insensitive to pancl shape and sizc.

\subsection{SOURCE PANEL METHOD APPLIED TO ELLIPTIC CONES}

Flows around non-axially-symmetric three-dimensional bodies in the form of elliptic cones were calculated using the source panel method. The second order theory of Van Dyke [21] was programmed and the pressure coefficient computed at the control points to compare with the source panel calculated values. Figure 32 shows the two pressure distributions on an 
elliptic cone with a maximum cone half-angle of $14^{\circ}$ and a ratio of minor to major axis of 0.532 at a Mach number of $\sqrt{2}$. Agreement is seen to be very good with the Van Dyke method yielding slightly higher values. Similar calculations are shown in Figure 33 for a maximum cone half-angle of $15^{\circ}$, a ratio of minor to major axis of 0.3 at a Mach number of $\sqrt{2}$. The agreement is excellent. For this case the source panel method gives slightly higher values on the flat side of the cone than the Van Dyke second order theory. Similar results are shown in figure 34 for a cone with a maximum half-angle of $18.4^{\circ}$ and a ratio of minor to major axis of $1 / 3$ at a Mach number of 1.81 . Figure 35 shows a graph of the pressure coefficient from a cone with a maximum half-angle of $30^{\circ}$ and a ratio of minor to major axis of 0.2 . Here, the panel method yields considerably lower pressure than the second order theory.

For the cone of $30^{\circ}$ maximum half-angle in figure 35 elliptic coordinates were used to obtain a finer paneling in the higher pressure region. For this paneling, a sharp dip in the pressure occurred in the region of maximum cone half-angle (see figure 36). This dip can be remedied by choosing the perturbation velocity vector $\bar{V}$ to be proportional to perturbation mass flux vector $\overline{\mathrm{W}}$ by the relation

$$
\overline{\mathrm{V}}=\overline{\mathrm{W}} / \rho, \text { with } \rho=1-\mathrm{M}^{2} \phi_{\mathrm{X}}+\ldots \text { for } \phi_{\mathrm{X}}<0
$$

This equals the correct velocity to the first order in the perturbation potential $\phi$ and when $-\phi_{X}$ gets large near stagnation points it gives better pressure results. The pressure distribution corrected by this formula using the isentropic pressure relation is shown by the triangles in figure 36. The pressure distribution is considerably improved, although the comparison with second order theory is not as good as for the more slender cones.

Figure 37 compares the calculated pressures obtained by using mass flux boundary conditions with those obtained by prescribing zero normal velocity boundary conditions and with experiments. For this example, velocity boundary conditions yield better agreement with expcriments (ref [22]). The linear solution with mass flux boundary conditions overpredict the pressure particularly on the flat side of the cone. Furthermore, the Van Dyke [21] second order theory tends to yield higher pressure as in the previous cases, than linearized theory with mass flux boundary conditions and, hence, also over preducts the actual pressures on elliptic cones.

\subsection{APPLICATION OF SOURCE AND DOUBLET PANEL METHODS TO PLANAR WINGS WITH LINEARIZED BOUNDARY CONDITIONS}

Source and doublet panels distributed on a wing planform were used to represent thickness and camber with linearized boundary conditions. Figure 38 shows the pressure coefficients for a yawed flat plate delta wing at angle of attack computed by the present method compared with the exact linearized theory solution in Jones and Cohen [23]. Agreement is seen to be good. However, the discontinuity in velocity gradient on the Mach line through the apex can be represented even better by a paneling which uses the Mach line emanating from the vortex as a network boundary. For this case (not shown in the figure) the pressure was exactly constant in the region between the supersonic leading edge and the Mach line through the corner as the theory predicts. 
The planar doublet panel method was tried on a wing of zero thickness with a parabolic arc camber profile described in figure 39. The pressure is in excellent agreement with exact linarized theory solution. Breaking the wing into networks bounded by Mach lines emanating from discontinuities in the wing leading edge allows for better representation of the infinte velocity gradient occurring at such Mach lines.

Using the same parabolic arc profile wing, the targential free stream perturbation velocity calculated from the theoretical solution was applied as design boundary conditions to a section of the wing between the supersonic leading edge and a radial line passing through the corner with the subsonic leading edge as seen in figure 40 . Analysis boundary conditions were applied to the remaining portion of the adjacent to the subsonic leading edge. The computed downwash from the panel method is compared with the actual wing slopes in figures 40 and 41 along the two lines numbered $N=1$ and 2 in the sketch. Figure 40 shows the greatest departure of the downwash from the actual wing slopes, and the match with actual slopes improves as one moves closer to the supersonic leading edge. It was found that the design method was unstable when the subsonic leading edge was included in the design network. However, these results represent only an initial attempt, and it is anticipated that these problems can be overcome.

With the same planform as in figure 39 and a symmetrical profile described by the same parabolic arc, the pressure distribution was obtained by the planar source panel method. Comparison with the exact solution is presented in figure 42 . The calculated results are in excellent agreement with the theory. Using the theoretical pressure distribution in the form of the tangential free stream perturbation velocity component as design-type boundary conditions in this case, yielded a downwash distribution which was undestinguishable from the actual wing slopes.

While using planar panels with linearized boundary conditions yields good comparison with exact linear theory solutions, a better comparison with experiment and with more exact supersonic solutions is obtained by paneling the wing surface and applying the exact boundary conditions of vanishing normal mass flux. Figure 43 compares the pressure distribution on a symmetric parabolic arc two dimensional airfoil by the present source panel method adapted for two dimensional flow with exact linear theory, the second order expansion (ref. [24]), and also with experiment. The present method with the pressure computed by the isentropic relation is in close agreement with second order theory on the upper surface and with experiment. The agreement on the lower surface is not quite so good but is still considerably better than linear theory.

\subsection{APPLICATION OF COMBINED SOURCE AND DOUBLET PANELS WITH POTENTIAL BOUNDARY CONDITIONS TO THREE DIMENSIONAL CONFIGURATIONS}

For configurations of irregular shape such as inlets, nacelles, and bodies, discontinuities in slope produce disturbances in the interior which reflect from the singularity sheet representing the configuration surface. These waves on repeated reflection may build up in strength and affect the surface singularity distribution which will in turn, introduce perturbations in the exterior pressure. These perturbations are spurious in that they are not properly related to physical conditions on the exterior surface where they occur. By using combined source and doublet paneling with both interior and exterior boundary conditions, the interior flow perturbations may be eliminated. Panel spacing requirements then depend only on the exterior 
geometry as does the true physical flow, without further spacing requirement arising from interior reflected waves.

For a closed body; this may be accomplished by setting the source distribution $\sigma$ equal to

$$
\sigma=-\overline{\mathrm{U}} \cdot \hat{\mathrm{n}}
$$

where $\bar{U}$ is the free stream velocity vector and $\hat{n}$ is the outward normal to the panel. The doublet distribution is then found by setting the perturbation velocity potential $\phi$ on the interior surface equal to zero at the panel control points.

A simple axially symmetric inlet was first analyzed by the source panel method. The pressure distribution along with the drawing of the inlet with its panel distribution is shown in figure 44. The result indicates that internal waves from the lip are influencing the pressure distribution on the outside of the nacelle. To eliminate the internal waves, a combined source and doublet distribution was applied to each panel. For this particular calculation, the $\phi=0$ interior boundary condition was replaced by the equivalent boundary condition of the vanishing of the normal component of the mass flux to the exterior surface. (Although the inlet is not closed, the $\phi=0$ interior boundary condition would work successfully for the isolated nacelle since $\phi$ is identically zero on the inlet opening in the absence of an incident perturbation flow.) The resulting pressure distribution, shown in figure 45 , is much smoother and in better agreement with the Lighthill [25] solution.

The combined source-doublet paneling method with exact boundary conditions was also applied to the Carlson [26], [27] wing 2T. The paneling is shown in figure 46. The pressure distribution on both upper and lower surfaces by the exact isentropic formula is plotted in figures 47 and 48 for four of the spanwise locations in figure 47 . Except in the tip trailing edge region the pressure distribution is smooth and compares favorably with experiment. The smoothness of the pressure coefficient indicates that with the combined source and doublet paneling there is little buildup of internal waves. (As we shall see later, the tip region pressures are improved with the nine parameter spline.)

\subsection{APPLICATION OF THE CONTINUOUS DOUBLET SPLINE TO THREE DIMENSIONAL CONFIGURATIONS}

The doublet spline used on each of the preceding examples was developed earlier for subsonic flow and is described in reference [3]. This spline utilized a quadratic distribution of the doublet strength over the plane panel obtained by projecting the four corners of the curved panel onto the average plane. The six parameters associated with the doublet strength allow the doublet strength either to be continuous across the panel edges only in a root mean square sense or to be continuous only at panel corners. Discontinuities in doublet strength and non-contiguous panel edges introduce vortex lines which in certain panel configurations may introduce large disturbances in the flow. The parabolic arc profile cambered swept wing of zero thickness used in figure 39 was analyzed with linearizcd boundary conditions but with different paneling. A portion of the wing paneling near the supersonic leading edge is shown in figure 49 . The pressure distribution along the 3 strips of panels are plotted in figures 50, 51 and 52. The six parameter doublet spline results exhibit large oscillations in the pressure coefficient near the tip. These oscillations are seen to be eliminated by using the 
improved panel method with continuous doublet strength and contiguous panels.

It should be noted that the poor results from the paneling in figure 49 is over a small portion near the leading edge of the same wing shown in figure 39 on which such good results were obtained. This indicates a sensitivity of the six parameter spline solution to different paneling of the same wing. The limitation of the study to the region in figure 49 was chosen because a simple analytic solution for the parabolic cambered wing was easy to obtain. The complete cambered swept wing was also computed for the paneling shown schematically in figure 53a. AC and CE are special Mach lines along which gradients in the velocity or pressure are discontinuous. The pressure in the region ACE was very smooth and in agreement with theory. In this region all the panel edges are sonic or supersonic and small discontinuities in doublet strength across panel edges do not affect the downwash. The results in ECD were also good. In $\mathrm{ABC}$ a spike in the pressure occurred at a control point near the point $\mathrm{B}$. This control point was found to lie along a Mach cone emanating from an upstream corner point and the spike was eliminated when the paneling was readjusted.

When the paneling in figure $53 \mathrm{~b}$ was used, unstable results were obtained near the tip of the wing. Refining the paneling by an order of magnitude as in figure 49 made the results worse as seen in figures 50,51 and 52 . The refined paneling increased the likelyhood that disturbances from edge vortices could impinge on control points. The only really effective cure was to use contiguous panels with continuous doublet strength.

To test the sensitivity of the nine parameter doublet spline, the supersonic flow over a $60^{\circ}$ flat plate delta wing at a Mach number of $\sqrt{2}$ and angle of attack $5.73^{\circ}$ was computed using the arbitrary, somewhat pathological, paneling shown in figure $54 \mathrm{a}$. The surface streamline slopes at the panel control points are plotted in figure $54 \mathrm{~b}$, and compared with the exact linearized solution in reference [23], page 157. The agreement with theory is good, with most of the spread occurring in the panels near the vertex.

The utilization of combined source and doublet panels on thick wings and fuselages eliminates interference of the interior wave reflection with the exterior pressure distribution by applying boundary conditions which eliminate the interior perturbation flow. The paneling shown in figure 55 was used to compute the flow over the forebody of the $\mathrm{B} 1 \mathrm{bom}$ ber. Use of the 6 coefficient doublet spline yielded smooth results and good agreement with experiment when the pressure coefficient was computed by means of the doublet gradient as seen in figure 56 . The pressure coefficient computed by the aerodynamic influence coefficients in figure 57 , however, should agree with the results in figure 56 , but shows considerable departure from the experiment (see ref. [28]). This deviation is attributed to the influence of the line vortices emanating from those panel edges lacking continuity of the doublet strength and from noncontiguous edges for which the singularities in the aerodynamic influence coefficients from adjacent panels do not cancel each other because of the gap even when the doublet strength is continuous. Calculations of pressure coefficient from the aerodynamic influence coefficients using the improved contiguous paneling and continuous doublet spline agree very well with the experimental results as seen in figure 58.

The two different panel methods were also applied to the Carlson wing using the paneling shown in figure 46. Since the leading and trailing edges are supersonic it is possible to analyze the pressures on the wing with sources only on the surface. Since there is no influence of the 
trailing vortex on the wing because of the supersonic trailing edge, the wake also can be ignored. The results for source paneling in figures 59 and 60 indicate an unstable solution resulting from the interior Mach wave reflections. From figures 47 and 48 we see that the combined source and doublet paneling with the six parameter spline show considerable improvement with disturbances from panel edge vortices only occurring in the strip 10 near the tip. The use of the continuous 9 parameter spline eliminates all disturbance and yields results in excellent agreement with experiment as shown in figures 61 and 62 .

An exact solution of the nonlinear inviscid equations for comparison purposes can be generated by using the exact solution for axisymmetric flow past a cone given in references [17] to [20]. Any plane which does not pass through the vertex of the cone will intersect the shock in an hyperbola (see figures 63 and 64). The shape of the surface was generated by computing the streamlines which pass through this hyperbola. The flow properties on this surface are the same as if the solid surface itself were immersed in the flow.

Results obtained by the 9 parameter spline for the surface defined in figure 64 were initially very disappointing (see figure 65). The problem is in the approximation of the sharp leading edge which has a slope of about 1 degree. When the configuration was taken to be the curved surface and the $x, y$ intersecting plane, control points near the leading edges of the two networks are displaced in different directions which proved to be more significant for the thin edges.

Much better results were obtained when the configuration was taken to be symmetric about the $x y$ intersecting plane (see figure 66). The errors at the top near the trailing edge $(x=1, y=0)$ are undoubtedly due to inadequate paneling in that vicinity, since the panel normals exhibit anomalous variations there. These variations are discussed in a subsequent paragraph. Were our interest not confined to the upper surface, symmetry would not be available to fix the problem in figure 65 . A more general approach is to set up a doublet network on the mean surface between the plane and the curved surface. This has been done with satisfactory results, as shown in figure 67. In this calculation the upper and lower surfaces were covered with both sources and doublets, with source strength specified to cancel the normal component of the free stream and the potential inside set equal to zero. The normal mass flux was set equal to zero on the interior mean surfacc.

To study in greater detail the difficulty with thin edges, we considered the flow past a sharp-edged delta wing whose lower surface is at zero angle of at tack and whose upper surfaces are at a small inclination to the freestream. For the case of a supersonic leading edge, the results obtained were poor near the leading edge and got worse downstream (see figure 68). Analysis of the results revealed a small discontinuity in doublet strength at the leading cdge junction of the upper and lower surface networks. This was eliminated by replacing the $\phi_{\ell}=0$ boundary conditions at the control points along the leading edge of one of the networks by $\overline{\mathrm{W}}_{\mathrm{u}} \cdot \hat{\mathrm{n}}=-\overline{\mathrm{U}} \cdot \mathrm{n}$. The results improved spectacularly, as shown in figure 69 . For the case of a subsonic leading edge, the quality of the solution, as evidenced by such data as the normal mass flux components on the external surfaces, also was much better when the boundary conditions were adjusted to insure continuity of the doublet strength from upper to lower surface. However, figure 70 shows that the pressure distribution was just as good whether or not the doublet strength was continuous. We attribute this difference in behavior to the difference in character between the supersonic and subsonic vortices generated at a doublet strength discontinuity. (These computations were made with the program that did not 
match doublet strength exactly across network edges. With the later program, these difficulties may not have arisen).

The remedy which worked so well with the delta wings improved the results for the conical-flow-field surface, but not to our complete satisfaction. Figure 71 shows the pressure distribution at the centers of panels along the leading edge to be quite oscillatory. Much better results have been obtained with different paneling of the same surface, and, pending further study, we are inclined to ascribe the inaccuracies shown in figure 71 to the high aspect ratio of the leading-edge panels.

Another problem encountered with the concial-flow-field surface was a fall off in pressure on the triangular panels near the top of the bump. We feel this is due to an anomalous variation of the directions of the normals to the panels. However, we now find it is not easy to avoid such anomalies in the vicinity of saddle-point-like behavior of the surface being paneled. In particular, it is not generally the case that the surface normals converge to the correct value at a saddle point as the grid is refined. Unfortunately, results for the pressure distribution are quite sensitive to any disparity in surface normal direction. The alternative panelings shown in figure 72 differ considerably in the variation of the $x$-component of the surface normal over the triangular panels, but even the one on the right is sufficiently bad to cause a considerable drop off in pressure near the top of the bump (although much less than for the case on the left).

Experience with the delta wing of figures 68 to 70 lead to an improved way of matching the doublet strength at network edges. On the downstream side of the common network boundary the boundary conditions of the vanishing of the lower peturbation velocity potential is replaced by zero mass flux penetration of the upper surface. This dramatically improved the delta wing pressures. It also improved the solution to the stream surface of figure 63 , but the results appear to be adversely affected by the high aspect ratio panels along the leading edge.

\subsection{APPLICATION OF THE CONTINUOUS DOUBLET SPLINE WITH POTENTIAL BOUNDARY CONDITIONS TO SUPERSONIC FLOW OVER WING-BODY CONFIGURATIONS}

The paneling used on the wing body combination described in reference [29] is shown in figure 73. The midwing position with zero angle of attack and yaw was chosen. The flow has, therefore, two planes of symmetry and paneling is required for only one quarter of the surface. The body consists of an ogive nose terminated by a circular cylinder. The wing has a symmetric NACA 65 A004 profile. The pressure distribution on the fore-body is shown in figure 74. The agreement with the experimental measurements is good. Figure 75 shows plots of pressure coefficients at $36 \%, 53 \%$, and $75 \%$ of semispan. The pressure distribution for the panel method is smooth and in fairly good agreement with the experimental measurements. Over most of the cross section profiles the computed pressures are somewhat higher than the experimental values. Near the leading edge the gradient of the pressure coefficient from the panel method is steeper than the measured distribution. This difference is due in part to inadequate paneling near the leading edge. The actual airfoil has a blunt leading edge while the paneling is necessarily sharp since the leading edge panels must be inclined at angles less than the Mach angle. 
The flow over an arrow wing-body combination using the paneling in figure 76 , for which no gaps appear between network edges, was computed. The pressure distribution on the wing is shown at various spanwise cross sections in figures 77 and 78. The calculations are in good agreement with the pressure measurements. The introduction of far-field aerodynamic influence coefficients reduced the computing time by about $40 \%$ over the solutions with all near-field aerodynamic influence coefficients. Experimental pressures on the upper surface near the leading edge at the $65 \%$ and $80 \%$ span locations are consistently higher than the theory. This is attributed to a leading edge vortex which has not been taken into account in the calculations.

An analysis was performed on a similar arrow wing-body configuration in figure 79 but featuring a twisted wing. The spanwise wing paneling was rearranged to obtain a match with the pressure orifice locations on the wind tunnel model. The wing body intersection paneling was also refined to match the model closely. The paneling resulted in 983 control points out of a possible 1000. The usual panel boundary conditions of $\phi=0$ on the interior surface and $\sigma=-\overline{\mathrm{U}}$. $\hat{\mathrm{n}}$ were applied. The solution required about $600 \mathrm{cpu}$ seconds on the CDC 7600 .

A comparison of wing surface pressure distribution with experiment is shown in figures 80 and 81 for a Mach number of 1.70 and 4 degrees angle of attack. Good agreement with experiment is shown except at the inboard most station. The leading edge peak in the calculations is caused by a disturbance at the intersection of the wing at the leading edge and the body. The effects of this disturbance are also seen in the calculated body pressures shown in figure 82. It is thought that this was caused by poor paneling near the wing-body intersection where one of the subpanels was found to be superinclined. Superinclined panels cannot represent solid surfaces.

\subsection{APPLICATION OF SUPERINCLINED PANELS TO CLOSE INLETS FOR THE COMPUTATION OF SUPERSONIC FLOW OVER NACELLES WITH THE COMBINED SOURCE AND DOUBLET PANEL METHOD}

To test the effectiveness of superinclined panels for closing an inlet, the supersonic flow over the nacelle shown in figure 83 was computed using the pilot code. A plane superinclined network was used to cover the inlet. The exit area need not be closed as in subsonic flow since it lies outside the region of influence for the nacelle.

Boundary conditions of vanishing perturbation potential and perturbation mass flux were applied to the downstream side of the inlet and conventional potential type boundary conditions were used on the nacelle surface. To provide a disturbance for the superinclined network to cancel, four source panels in the plane $\phi=\pi / 2$ were placed upstream a distance of the nacelle radius. To have a solution without superinclined panels for comparison, the source distribution on the nacelle was set equal to $-\overline{\mathrm{U}} \cdot \hat{\mathrm{n}}$ and standard mass flux boundary conditions were applied to the exterior surface. Figures 84 and 85 show the pressure coefficient along four aximuth positions on the nacelle. The two calculations are in good agreement. The flow without the superinclined panels appears to have interference from the interior flow due to the reflected waves from the lip that is effectively eliminated by the superinclined panels.

Another test on how effectively the superinclined networks absorb upstream disturbances was conducted on the same nacelle. The plane superinclined network was placed inside the nacelle at axial station 2.25 (see figure 86 ). On the upstream portion of the nacelle, zero 
normal mass flux boundary conditions were prescribed on the upper surface of a source network. This results in an internal flow to be absorbed by the superinclined network.

Figurc 86 shows the exterior surface pressure distribution on the nacelle as a function of axial position for two different paneling of the superinclined network. The 48 panel network is seen to be too coarse to absorb adequately the interior reflections. The pressure is oscillatory with a wavelength about equal to the reflected Mach wave pattern. Doubling the panels by increasing the number of radial divisions smooths out the pressure distribution. Except for the region near the superinclined network, the pressure is in good agreement with Lighthill's theory [25].

With the successful development of the superinclined networks, the LES 216 supercruiser configuration, which contains superinclined inlets, was successfully analyzed by the advanced panel pilot code. The paneling scheme is shown in figures 87 and 88 . A total of 674 panels divided into 31 networks were used to define the configuration. The model has two openings for flow into the engine. The diverter, which splits off the low energy of the boundary layer flow from the engine inlet is closed by a superinclined network with the mass flux prescribed as boundary conditions. The boundary of the inlet opening has edges which are subinclined to the flow on the upper part and superinclined to the flow on the lower part. The upper section of the inlet is covered by a subinclined network and the lower side is covered with a superinclined network and the mass flux is specified on both networks. The complete solution required $612 \mathrm{cpu}$ seconds on the CDC 7600 . Computed results are compared with experimental data and with results from a linearized boundary condition method similar to FLEXSTAB are shown in figure 89 . There is very good agreement between the pilot code calculations and the experimental data of reference [31]. Note that the results from the linearized code, in which the fuselage is represented by a simple body of revolution, are clearly inadequate to rcpresent the effects of the canopy and inlet on the wing pressures. Here is an example in which the present panel method can more accurately model the aerodynamics of the configuration than the simpler models currently in use.

Some waviness is apparent in the pressure distribution on the upper surface for $\eta=0.37$. This was observed in other pressure distributions and was found to be eliminated by increased weighting of the upstream panel center values of the singularity strengths in the least square fit. This is explained in Appendix $\mathrm{C}$ where a detailed description of the singularity splines is presented. 


\subsection{CONCLUSIONS}

Theoretical analysis of the singularities caused by discontinuities in doublet strength and in panel geometry, combined with the experience of the flow computations described in section 12 , indicate that it is important to have panel geometry that contains no gaps and a doublet spline system that maintains continuity of doublet strength over the surface. It was also found that use of a source panel system alone on closed configurations, such as three dimensional wings and bodies, produces interior wave reflections that influence the exterior pressure distribution.

This ultimately lead to the development of the present panel method utilizing combined source and doublet panels with potential boundary conditions to eliminate the interior perturbations. The panel geometry with all contiguous edges was achieved by dividing the basic nonplanar quadrilateral panel into eight triangular subpanels. Continuity of doublet strength was obtained by prescribing a quadratic doublet distribution over each triangular subpanel instead of just over the panel projection. The resulting panel method appears to be insensitive to choice of paneling provided the paneling is sufficiently fine to describe the solution adequately.

Superinclined panels, that is, panels which are inclined at angles with the free stream greater than the Mach angle, were developed and applied to the closing of inlets on nacelles. They were found to be effective in absorbing the incident perturbation flow. With the addition of superinclined panels, the present advanced panel method is now capable of solving for the flow over complicated wing-body combinations with engine inlets and nacelles.

Boeing Commercial Airplane Company

P.O. Box 3707

Seattle, Washington 98124

1978 


\section{APPENDIX A: \\ DERIVATION OF BASIC FUNCTIONS AND THEIR DERIVATIVES}

\section{A1. BASIC VARIABLES FOR SUBINCLINED PANELS (SECTIONS 5 TO 8)}

For convenience, we have introduced the following parameters:

$$
\mathrm{m}=\left(\mathrm{y}_{2}-\mathrm{y}_{1}\right) /\left(\mathrm{x}_{2}-\mathrm{x}_{1}\right) \quad \lambda=1 / \mathrm{m}
$$

The quantity $m$ is the slope of the panel edge defined by

$$
x_{m}=\left(x-x_{1}\right)-\left(y-y_{1}\right) / m=0
$$

We let

$$
\begin{aligned}
& s_{m}=x_{m}+s / m \\
& r=\sqrt{s^{2}+z^{2}} \\
& s_{z}=s x_{m}-z^{2} / m \\
& z_{m}=x_{m^{2}}+z^{2} / m^{2}
\end{aligned}
$$

\section{A2. BASIC INTEGRALS FOR SUBINCLINED PANELS}

For the basic integrals we have defined

$$
\begin{aligned}
I_{n}(r, t) & =\int t_{d t} / \sqrt{t(t+2 r)} \\
w_{n} & =\int s^{n} d s / \sqrt{s_{m^{2}}-r^{2}}=\int s^{n_{d s} / R} \\
Q_{n} & =\int s^{n} / r^{2} R \\
R_{n} & =\int s^{n} R d s \\
P_{n} & =z \int s^{n} R d s / r^{2} \\
S_{n} & =\int s^{n} I_{0}\left(r, s_{m}-r\right) d s
\end{aligned}
$$


We shall use the subscript $m$ to denote combinations of functions of the form

$$
w_{m n}=\int \frac{s_{m} s^{n}}{\sqrt{s_{m^{2}}-r^{2}}} d s=x_{m} w_{n}+w_{n+1} / m
$$

and similarly for the other functions $\mathrm{Q}, \mathrm{R}$, and $\mathrm{S}$.

\section{A3. RECURSION RELATIONS FOR THE FUNCTIONS-SUBINCLINED PANELS}

The integral $I_{n}(r, t)$ occurs in the first integration of $\phi$ in the form

$$
\int_{0}^{s_{m}-r} t^{n} d t / \sqrt{t(t+2 r)}=I_{n}\left(r, s_{m}^{-r}\right)
$$

To find a recursion formula, we consider

$$
\frac{d}{d t}\left(t^{n} \sqrt{t(t+2 r)}\right)=\frac{t^{n}(r+t)+n t^{n}(t+2 r)}{\sqrt{t(t+2 r)}}
$$

Integration yields

$$
t^{n} \sqrt{t(t+2 r)}=r(2 n+1) I_{n}(r, t)+(n+1) I_{n+1}(r, t)
$$

or

$$
I_{n+1}(r, t)=\left[t^{n} \sqrt{t(t+2 r)}-r(2 n+1) I_{n}(r, t)\right] /(n+1)
$$

Substituting the limits 0 to $\mathrm{s}_{\mathrm{m}^{-\mathrm{r}} \text { yields }}$

$$
I_{n+1}\left(r, s_{m}-r\right)=\left[\left(s_{m}-r\right) n \sqrt{s_{m}^{2}-r^{2}}-(2 n+1) r I_{n}\left(r, s_{m}-r\right)\right] /(n+1)
$$

When the first three $I_{n}$ functions are expressed in terms of $I_{0}$, we obtain 


$$
\begin{aligned}
& I_{1}=R-r I_{0} \\
& I_{2}=3 r^{2} I_{0} / 2+\left(s_{m}-4 r\right) R / 2 \\
& I_{3}=-5 r^{3} I_{0} / 2+\left(2 s_{m}{ }^{2}-9 s_{m} r+22 r^{2}\right) R / 6
\end{aligned}
$$

where $R=\sqrt{s_{m}^{2}-r^{2}}$

Following a similar procedure for the $w_{n}$ we write

$$
\frac{d}{d s}\left(s^{n} \sqrt{s_{m^{2}}-r^{2}}\right)=\frac{n s^{n-1}\left(s_{m}^{2}-r^{2}\right)+s^{n}\left(s_{m} / m-s\right)}{\sqrt{s_{m^{2}-r}}}
$$

Integrating and rearranging terms yields

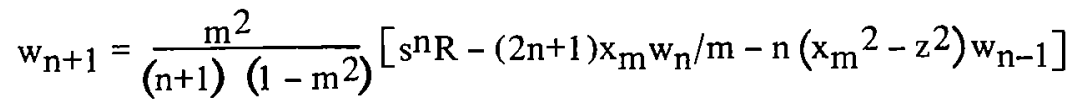

Now

$$
Q_{n}=\int \frac{s^{n} d s}{r^{2} \sqrt{s_{m}^{2}-r^{2}}}=\int \frac{s^{n-2} d s}{\sqrt{s_{m}^{2}-r^{2}}}-z^{2} \int \frac{s^{n-2} d s}{r^{2} \sqrt{s_{m}^{2}-r^{2}}}
$$

and this results in

$$
\mathrm{Q}_{\mathrm{n}}=\mathrm{w}_{\mathrm{n}-2}-\mathrm{z}^{2} \mathrm{Q}_{\mathrm{n}-2}
$$

The function $R_{n}$ in equation (A4) is more easily expressed in terms of the other functions by moving the radical to the denominator. This yields

$$
R_{n}=w_{m m n}-w_{n+2}-z^{2} w_{n}
$$


Expanding $\mathrm{w}_{\mathrm{mm0}}$ and using the recursion for $\mathrm{w}_{2}$, we obtain for $\mathrm{R}_{0}$

$$
\mathrm{R}_{0}=\left(\mathrm{x}_{\mathrm{m}}^{2}-\mathrm{z}^{2}\right) \mathrm{w}_{0} / 2+\mathrm{x}_{\mathrm{m}} \mathrm{w}_{1} / 2 \mathrm{~m}
$$

The functions $S_{n}$ are easily expressed in terms of the other functions by integration by parts. Hence, using equation (A14), we get

$$
\begin{aligned}
S_{n} & =\int s^{n} I_{0}\left(r, s_{m}-r\right) d s=\int s^{n_{l o g}}\left[\left(s_{m}+R\right) /\left(s_{m}-R\right)\right] d s \\
& =\left[s^{n+1} I_{0}\left(r, s_{m}-r\right)+Q_{m n+2}-w_{n+1} / m\right] /(n+1)
\end{aligned}
$$

Using equation (A10), we may simplify this expression to

$$
S_{n}=\left[s^{n+1} I_{0}+x_{m} w_{n}-z^{2} Q_{m n}\right] /(n+1)
$$

The $P_{n}$ functions can be expressed in terms of the functions $Q_{n}$ and $w_{n}$ since

$$
P_{n}=z \int s^{n} R d s / r^{2}=z \int^{n}\left(s_{m^{2}}-r^{2}\right) d s / R r^{2}
$$

then

$$
P_{n}=z\left(Q_{m m n}-w_{n}\right)=z\left(x_{m} Q_{m n}+Q_{m n+1} / m-w_{n}\right)
$$

As only the functions $\mathrm{P}_{0}$ and $\mathrm{P}_{1}$ occur, we write these down immediately using the recursion formula for the $Q_{n}$ in equation (A10)

$$
P_{0}=x_{m}\left(z Q_{m 0}\right)+z Q_{m 1} / m-z_{0}
$$

and

$$
\mathrm{P}_{1}=\mathrm{x}_{\mathrm{m}}\left(\mathrm{zQ}_{\mathrm{ml} 1}\right)+\mathrm{z} \cdot\left[\mathrm{x}_{\mathrm{m}} \mathrm{w}_{1}-\mathrm{z}^{2} \mathrm{Q}_{\mathrm{m} 10}+\mathrm{w}_{1} / \mathrm{m}\right]-\mathrm{zw}_{1}
$$




\section{A4. ZERO ORDER FUNCTIONS}

Evaluating the integral for $\mathrm{I}_{0}$ yields

$$
I_{0}\left(r, s_{m}-r\right)=\int_{0}^{s_{m}-r} \frac{d t}{\sqrt{t(t+2 r)}}=\frac{1}{2} \log \left\lceil\frac{s_{m}+R}{s_{m}-R}\right\rceil
$$

In this and all subsequent integrals, a form is chosen so that the functions vanish when the radical $\mathrm{R}=\sqrt{\mathrm{s}_{\mathrm{m}}{ }^{2}-\mathrm{r}^{2}}$ vanishes and, hence, the real part is zero for $\mathrm{R}$ imaginary. Similarly

$$
\begin{aligned}
\mathrm{w}_{0}\left(\mathrm{~s}, \mathrm{~s}_{\mathrm{m}}, \mathrm{r}, \mathrm{m}\right) & =\int \frac{\mathrm{ds}}{\sqrt{\mathrm{s}_{\mathrm{m}^{2}-\mathrm{r}^{2}}}} \\
& =\frac{\mathrm{m}}{2 \sqrt{1-\mathrm{m}^{2}}} \log \left[\frac{\left(\mathrm{s}_{\mathrm{m}}-\mathrm{ms}\right)+\mathrm{R} \sqrt{1-\mathrm{m}^{2}}}{\left(\mathrm{~s}_{\mathrm{m}}-\mathrm{ms}\right)-\mathrm{R} \sqrt{1-\mathrm{m}^{2}}}\right]
\end{aligned}
$$

for $|\mathrm{m}|<1$ and

$$
\begin{aligned}
& \mathrm{w}_{0}=\frac{\mathrm{m}}{\sqrt{\mathrm{m}^{2}-1}} \tan ^{-1}\left(\frac{\mathrm{ms}-\mathrm{s}_{\mathrm{m}}}{\mathrm{R} \sqrt{\mathrm{m}^{2}-1}}\right) \\
& \mathrm{w}_{0}=\frac{1}{\sqrt{1-\lambda^{2}}} \tan ^{-1}\left(\frac{\mathrm{s}-\lambda \mathrm{s}_{\mathrm{m}}}{\mathrm{R} \sqrt{1-\lambda^{2}}}\right)
\end{aligned}
$$

for $|m|>1$. Care must be taken in evaluating all inverse tangents in the aerodynamic influence coefficients to select the correct quadrant. The correct signs of the numerator and denominator of the argument are essential. In the FORTRAN code the function ATAN2(A, B) is used instead of $\operatorname{ATAN}(A, B)$.

It is considerably more complicated to derive $Q_{0}$ and $Q_{1}$. For this we write

$$
\frac{1}{s+i z}=\frac{s-i z}{r^{2}}, i=\sqrt{-1}
$$

and obtain

$$
I_{Q}=Q_{1}-i z Q_{0}=\int \frac{d s}{(s+i z) \sqrt{s_{m^{2}-r^{2}}}}
$$


Introducing a new variable $\tau=\mathrm{s}+\mathrm{iz}$, the integral $\mathrm{I}_{\mathrm{Q}}$ becomes

$$
\mathrm{I}_{\mathrm{Q}}=\int \frac{\mathrm{d} \tau}{\tau \sqrt{\mathrm{a}+\mathrm{b} \tau+c \tau^{2}}}=-\frac{1}{2 \sqrt{\mathrm{a}}} \log \left[\frac{\mathrm{a}+\mathrm{b} \tau / 2+\mathrm{R} \sqrt{\mathrm{a}}}{\mathrm{a}+\mathrm{b} \tau / 2-\mathrm{R} \sqrt{\mathrm{a}}}\right]
$$

where $\quad a=\left(x_{m}-i z / m\right)^{2}, b=2\left(x_{m}-i z / m\right) / m+2 i z$, and $c=\left(1-m^{2}\right) / m^{2}$.

Substituting $a, b$, and $c$ and simplifying yield

$$
I_{Q}=-\frac{1}{2\left(x_{m}-i z / m\right)} \log \left[\frac{x_{m}\left(s_{m}+R\right)-z^{2}+i z\left(y_{m}-R / m\right)}{x_{m}\left(s_{m}-R\right)-z^{2}+i z\left(y_{m}+R / m\right)}\right]
$$

where $y_{m}=s-s_{m} / m$. Multiplying by $x_{m}-i z / m$ and using the recursion formula for $Q_{n}$ leads to

$$
\begin{gathered}
\mathrm{Q}_{\mathrm{m} 1}-\mathrm{w}_{0} / \mathrm{m}-\mathrm{iz} \mathrm{Q}_{\mathrm{m} 0} \\
=-\frac{1}{2} \log \left(\frac{\mathrm{z}_{\mathrm{cp}}}{\mathrm{z}_{\mathrm{cm}}}\right)
\end{gathered}
$$

It is convenient to eliminate all parts of $\mathrm{Q}_{\mathrm{m} 1}$ which depend only upon endpoints, i.e., only upon $s$ and $s_{m}$, since at endpoints $s$ and $s_{m}$ reduce to $y-y_{i}$, and $x-x_{i}, i=1,2$. We shall show that the function

$$
\frac{1}{2} \log \left|\frac{z_{c p}}{z_{c m}}\right|
$$

vanishes in the integration around a contour. Now

$$
\mathrm{Q}_{\mathrm{m} 1}-\mathrm{w}_{0} / \mathrm{m}=\int \frac{\mathrm{s}_{\mathrm{m}} \mathrm{sds}}{\mathrm{r}^{2} \sqrt{\mathrm{s}_{\mathrm{m}}^{2}-\mathrm{r}^{2}}}-\int \frac{\mathrm{ds}}{\mathrm{m} \sqrt{\mathrm{s}_{\mathrm{m}}^{2}-\mathrm{r}^{2}}}
$$

Since $s_{m}-s / m=0$ represents the panel edge, then $d s / m=d s_{m}$ along the panel edge; we have

$$
Q_{m 1}-w_{G} / m=\int\left[\left(\frac{s_{m} s}{r^{2} R}\right) d s-\frac{d s_{m}}{R}\right]
$$


In order for the integral to depend only upon its end points we must have

$$
\frac{\partial}{\partial s_{m}}\left(\frac{s_{m} s}{r^{2} R}\right)=-\frac{\partial}{\partial s}\left(\frac{1}{R}\right)
$$

That this is so is easily shown by carrying out the differentiation. Thus, when $Q_{m 1}$ is not multiplied by $m$ or $x_{m}$ the function $Q_{m 1}$ may be replaced by

$$
\mathrm{Q}_{\mathrm{m} 1}=\mathrm{w}_{0} / \mathrm{m}
$$

A similar approach may also be used to simplify $\mathrm{zQ}_{\mathrm{m} 0}$. Consider

$$
\begin{aligned}
Q_{I} & =\int \frac{z x_{m} d s}{\left(s_{m}^{2}-s^{2}\right) R} \\
& =\tan ^{-1}\left[\frac{z\left(s-\lambda s_{m}\right)}{x_{m} R}\right] \text { for }|m|>1 \\
& =\operatorname{sign}(z) \tan ^{-1}\left[\frac{m_{m} R}{-|z|\left(m s-s_{m}\right)}\right] \text { for }|m|<1
\end{aligned}
$$

Then

$$
z Q_{m 0}-Q_{I}=z \int \frac{s_{m} d s}{r^{2} R}-z \int \frac{x_{m} d s}{\left(s_{m^{2}}^{2}-s^{2}\right) R}
$$

Substituting $x_{m}=s_{m}-s / m$ and writing $d s / m=d s_{m}$ yield

$$
\begin{aligned}
z Q_{m 0}-Q_{I}= & z \int\left\{\left[\frac{s_{m}}{r^{2}}-\frac{s_{m}}{\left(s_{m}^{2}-s^{2}\right) R}\right] d s\right. \\
& \left.+\left[\frac{s}{\left(s_{m}^{2}-s^{2}\right) R}\right] d s_{m}\right\}
\end{aligned}
$$


It is easily shown that the line integral on the right hand side vanishes when integrated around a closed contour since

$$
\frac{\partial}{\partial s_{m}}\left[\frac{s_{m}}{r^{2} R}-\frac{s_{m}}{\left(s_{m}^{2}-s^{2}\right) R}\right]=\frac{\partial}{\partial s}\left[\frac{s}{\left(s_{m^{2}}^{2}-s^{2}\right) R}\right]
$$

Hence, we may replace $z Q_{m 0}$ by the simpler relation $Q_{I}$ when $z Q_{m 0}$ is not multiplied by $\mathrm{m}$ or $\mathrm{x}_{\mathrm{m}}$, i.e.

$$
\mathrm{zQ}_{\mathrm{m} 0}=\mathrm{Q}_{\mathrm{I}}
$$

In evaluating the functions $P_{0}$ and $P_{1}$ defined in equations (A13), we are not free to use the simpler versions of $\mathrm{zQ}_{\mathrm{m} 0}$ and $\mathrm{Q}_{\mathrm{m} 1}$ since these quantities are multiplied by $\mathrm{x}_{\mathrm{m}}$ and $1 / \mathrm{m}$ and, hence, do not cancel in integration around a panel corner. The complete function $\mathrm{zQ}_{\mathrm{m} 0}$ is found by taking the imaginary part of equation (A17). For subsonic panel edges, $\mathrm{m}<1$, we write $\mathrm{zQ}_{\mathrm{m} 0}$ in the form

$$
z_{\mathrm{m} 0}=-\frac{1}{2}\left\{\tan ^{-1}\left[\frac{\mathrm{z}\left(\mathrm{y}_{\mathrm{m}}-\mathrm{R} / \mathrm{m}\right)}{\mathrm{z}^{2}-\mathrm{x}_{\mathrm{m}}\left(\mathrm{s}_{\mathrm{m}}+\mathrm{R}\right)}\right]-\tan ^{-1}\left[\frac{\mathrm{z}\left(\mathrm{y}_{\mathrm{m}}+\mathrm{R} / \mathrm{m}\right)}{\mathrm{z}^{2}-\mathrm{x}_{\mathrm{m}}\left(\mathrm{s}_{\mathrm{m}}-\mathrm{R}\right)}\right]\right\}
$$

For supersonic panel edges it is convenient to combine the inverse tangents. Thus

$$
\mathrm{zQ}_{\mathrm{m} 0}=\frac{1}{2} \tan ^{-1}\left[\frac{2 \mathrm{zR}\left(\mathrm{x}_{\mathrm{m}} \mathrm{s}^{\left.-\mathrm{z}^{2} / \mathrm{m}\right)}\right.}{2 \mathrm{z}^{2} \mathrm{R}^{2}-\left(\mathrm{x}_{\mathrm{m}}{ }^{2}+\mathrm{z}^{2} / \mathrm{m}-\mathrm{z}^{2}\right)\left(\mathrm{s}^{2}+\mathrm{z}^{2}\right)}\right]
$$

For points outside the Mach cone, $R$ is set to 0 but inside the Mach wedge of figure 90 defined by the envelope of Mach cones from points on the supersonic edge for which

$$
\mathrm{x}_{\mathrm{m}}{ }^{2}+\mathrm{z}^{2} / \mathrm{m}^{2}-\mathrm{z}^{2}>0
$$

the function $\mathrm{zQ}_{\mathrm{m} 0}$ takes on the values $\pm \pi / 2$.

The function $Q_{m 1}$ is found by taking the real part of equation (A17). The result can be simplified since a common factor

$$
\mathrm{x}^{2}+\mathrm{z}^{2} / \mathrm{m}^{2}-\mathrm{z}^{2}
$$

can be taken out of the numerator and denominator of the logarithm argument. The result is

$$
\mathrm{Q}_{\mathrm{m}_{1}}=\mathrm{w}_{0} / \mathrm{m}-\mathrm{I}_{0}\left(\mathrm{~s}_{\mathrm{m}}, \mathrm{R}\right)
$$

where $I_{0}$ is defined in equation (A14). 


\section{A5. EXPANSIONS OF $w_{0}$ AND $w_{1}$ FOR MACH LINE PANEL EDGES}

When $|\mathrm{m}|=1$, the panel edge is aligned with a Mach line. The coefficient of the log term and arc tangent term in $w_{0}$ then becomes infinite and an expansion is needed for both $w_{0}$ and $w_{1}$. From equation (A15) we write

where $\beta_{\mathrm{m}}=\sqrt{1-\mathrm{m}^{2}}$ and $\mathrm{z}_{\mathrm{R}}=\mathrm{R} /\left(\mathrm{s}_{\mathrm{m}}-\mathrm{ms}\right)$. Since $\beta_{\mathrm{m}}$ is small, the logarithm term is expanded in the form

$$
\mathrm{w}_{0}=\mathrm{mz}_{\mathrm{R}}{ }^{2}\left[1+\mathrm{z}_{\mathrm{R}}^{2}\left(1-\mathrm{m}^{2}\right) / 3+\mathrm{z}_{\mathrm{R}}^{4}\left(1-\mathrm{m}^{2}\right)^{2 / 5}+\mathrm{z}_{\mathrm{R}} 6\left(1-\mathrm{m}^{2}\right)^{3} / 7+\ldots\right]
$$

This expansion is valid for $\mid \mathrm{m} /$ greater or less than 1 . For $\mathrm{m}= \pm 1$, it reduces to

$$
\mathrm{w}_{0}=\mathrm{R} /\left(\mathrm{s}_{\mathrm{m}} \pm \mathrm{s}\right)
$$

To obtain a similar expansion for $\mathrm{w}_{1}$ we consider equation (A9) and obtain

$$
\mathrm{w}_{1}=\mathrm{m}^{2}\left[\mathrm{R}-\mathrm{x}_{\mathrm{m}} \mathrm{w}_{0} / \mathrm{m}\right] /\left(1-\mathrm{m}^{2}\right)
$$

Substituting the expansion for $w_{0}$ yields

$\mathrm{w}_{1}=\frac{\mathrm{m}^{2}}{1-\mathrm{m}^{2}}\left\{\mathrm{R}-\mathrm{x}_{\mathrm{m}^{2} \mathrm{R}}\left[1+\mathrm{z}_{\mathrm{R}}^{2}\left(1-\mathrm{m}^{2}\right) / 3+\mathrm{z}_{\mathrm{R}}^{4}\left(1-\mathrm{m}^{2}\right)^{2} / 5+\mathrm{z}_{\mathrm{R}} 6\left(1-\mathrm{m}^{2}\right)^{3 / 7+\ldots}\right]\right.$

Now

$$
\begin{array}{r}
R-x_{m^{2}}=R\left[1-\frac{x_{m}}{s_{m}-m s}\right]=R\left[1-\frac{s_{m}-s / m}{s_{m}-m s}\right] \\
=\frac{\left(1-m^{2}\right) R s}{m\left(s_{m}-m s\right)}=\frac{\left(1-m^{2}\right) z_{R s}}{m}
\end{array}
$$

then $\mathrm{w}_{1}$ becomes

$$
\begin{aligned}
w_{1}= & m z_{R}\left\{s-m x_{m} z_{R}^{2}\left[1 / 3+z_{R}^{2}\left(1-m^{2}\right) / 5\right.\right. \\
& \left.\left.+z_{R}^{4}\left(1-m^{2}\right)^{2} / 7+z_{R} 6\left(1-m^{2}\right)^{3} / 9+\ldots .\right]\right\}
\end{aligned}
$$


Similar expansions may be derived for $w_{n}$ for $n>1$, but these functions are not required in the aerodynamic influence coefficients in the form now being used.

\section{A6. EFFICIENT COMPUTATION OF $w_{0}, w_{1}$ AND $Q_{I}$}

In the calculation of the influence coefficients, the functions $\mathrm{w}_{0}, \mathrm{w}_{1}$, and $\mathrm{Q}_{\mathrm{I}}$ are evaluated for each edge at the two endpoints. This involves the utilization of the computer subroutine for inverse tangent and logarithm twice for each edge. Considerable speed of computation can be achieved by combining the arctangents and logarithms for each edge. For the supersonic edge we have

$$
\mathrm{w}_{0}=\left.\frac{1}{\sqrt{1-\lambda^{2}}} \tan ^{-1}\left(\frac{-\mathrm{y}_{\mathrm{m}}}{\mathrm{R} \sqrt{1-\lambda^{2}}}\right)\right|_{1} ^{2}
$$

where $y_{m}=\lambda s_{m}-s$. Using the formula for the tangent of the difference of two angles yields

$$
w_{0}=\frac{1}{\sqrt{1-\lambda^{2}}} \tan ^{-1}\left(\frac{\sqrt{1-\lambda^{2}}\left(y_{m 1} R_{2}-y_{m 2} R_{1}\right)}{y_{m 1} y_{m 2}+\left(1-\lambda^{2}\right) R_{1} R_{2}}\right)
$$

where the subscripts denote the quantities at the designated endpoints of the edge. Similarly, for $\mathrm{Q}_{\mathrm{I}}$ we have

$$
\begin{aligned}
\mathrm{Q}_{\mathrm{I}} & =\left.\tan ^{-1}\left(\frac{-\mathrm{zy}_{\mathrm{m}}}{\mathrm{x}_{\mathrm{m}} \mathrm{R}}\right)\right|_{1} ^{2} \\
& =\tan ^{-1}\left(\frac{z \mathrm{x}_{\mathrm{m}}\left(\mathrm{y}_{\mathrm{m} 1} \mathrm{R}_{2}-\mathrm{y}_{\mathrm{m} 2} \mathrm{R}_{1}\right)}{\mathrm{x}_{\mathrm{m}}{ }^{2} \mathrm{R}_{1} \mathrm{R}_{2}+\mathrm{z}^{2} \mathrm{y}_{\mathrm{m} 2} \mathrm{y}_{\mathrm{m} 1}}\right)
\end{aligned}
$$


For subsonic edges

$$
\begin{aligned}
w_{0} & =\left.\frac{m}{2\left(1-m^{2}\right)} \log \left[\frac{s_{m}-m s+R \sqrt{1-m^{2}}}{s_{m}-m s-R \sqrt{1-m^{2}}}\right]\right|_{1} ^{2} \\
& =\frac{m}{\left(1-m^{2}\right)} \log \left(s_{m}-m s+R \sqrt{1-m^{2}}\right)-\left.\log \left(m^{2} z_{m}\right)\right|_{1} ^{2}
\end{aligned}
$$

Since $\mathrm{z}_{\mathrm{m}}$ (equation (A3)) has the same value at all points of the line, we obtain

$$
w_{0}=\frac{m}{\sqrt{1-m^{2}}} \log \left[\frac{\hat{y}_{m 2}+\sqrt{1-m^{2}} R_{2}}{\hat{y}_{m 1}+\sqrt{1-m^{2}} R_{1}}\right]
$$

where $\hat{y}_{m}=s_{m}-m s$. For $Q_{I}$ we have

$$
\begin{aligned}
\mathrm{Q}_{1} & =\left.\operatorname{sign}(\mathrm{z}) \tan ^{-1}\left[\frac{\hat{\mathrm{x}}_{\mathrm{m}} \mathrm{R}}{\mid \mathrm{z} ! \hat{\mathrm{y}}_{\mathrm{m}}}\right]\right|_{1} ^{2} \\
& =\tan ^{-1}\left[\frac{\mathrm{z} \hat{\mathrm{x}}_{\mathrm{m}}\left(\hat{\mathrm{y}}_{\mathrm{m} 1} \mathrm{R}_{2}-\hat{\mathrm{y}}_{\mathrm{m} 2} \mathrm{R}_{1}\right)}{\mathrm{z}^{2} \hat{\mathrm{y}}_{\mathrm{m} 1} \hat{\mathrm{y}}_{\mathrm{m} 2}+\hat{\mathrm{x}}_{\mathrm{m}} \mathrm{R}_{1} \mathrm{R}_{2}}\right]
\end{aligned}
$$

For edges aligned with the Mach lines, wo becomes singular and we must expand the function in powers of $\sqrt{1-\lambda^{2}} \quad$ Letting

$$
z_{r}=\frac{y_{m 1} R_{2}-y_{m 2} R_{1}}{y_{m 1} y_{m 2}+\left(1-\lambda^{2}\right) R_{1} R_{2}}
$$

and expanding the inverse tangent in $w_{0}$ yields

$$
w_{0}=z_{r}\left[1-\frac{\left(1-\lambda^{2}\right) z_{r}^{2}}{3}+\frac{\left(1-\lambda^{2}\right) 2 z_{r}^{4}}{5}-\frac{\left(1-\lambda^{2}\right) 3_{z_{r}}^{6}}{7}+\ldots\right]
$$


A similar expansion for $\mathrm{w}_{1}$ yields

$$
\begin{aligned}
\mathrm{w}_{1} & =\frac{1}{1-\lambda^{2}}\left[\lambda \mathrm{x}_{\mathrm{m}} \mathrm{w}_{0}-\mathrm{R}_{2}+\mathrm{R}_{1}\right] \\
& =\frac{1}{1-\lambda^{2}}\left[\lambda \mathrm{x}_{\mathrm{m}_{\mathrm{r}}}-\mathrm{R}_{2}+\mathrm{R}_{1}\right]-\mathrm{z}_{\mathrm{r}}\left[\frac{\mathrm{z}_{\mathrm{r}}^{2}}{3}-\frac{\left(1-\lambda^{2}\right) \mathrm{z}_{\mathrm{r}}^{4}}{5}+\frac{\left(1-\lambda^{2}\right){ }^{2} \mathrm{z}_{\mathbf{r}}{ }^{6}}{7}-\ldots\right]
\end{aligned}
$$

Substituting for $\mathrm{z}_{\mathrm{T}}$ and simplifying yields

$$
\begin{aligned}
w_{1} & =\left[\left(y_{m 1} s_{2}-R_{1}^{2}\right) R_{2}-\left(y_{m 1} s_{1}-R_{2}^{2}\right) R_{1}\right] /\left[y_{m 2} y_{m 1}+\left(1-\lambda^{2}\right) R_{1} R_{2}\right] \\
& -z_{r}\left[\frac{z_{r}^{2}}{3}-\frac{\left(1-\lambda^{2}\right) z_{r}^{4}}{5}+\frac{\left(1-\lambda^{2}\right){ }^{2} z_{r}^{6}}{7}-\ldots\right]
\end{aligned}
$$

The functions $\mathrm{w}_{0}$ and $\mathrm{w}_{1}$ are seen to exist for the value $|\lambda|=|\mathrm{m}|=1$.

\section{A7. GRADIENTS OF THE BASIC FUNCTIONS FOR SUBINCLINED PANELS}

The functions used in the influence coefficients are functions of $\mathrm{s}, \mathrm{s}_{\mathrm{m}}$, and $\mathrm{z}$ when $\mathrm{x}_{\mathrm{m}}=$ $s_{m}-s / m$ is substituted. When the limits $y-y_{1}$ and $y-y_{2}$ are substituted for $s$ into the functions for evaluation at the end points of the panel edge, the quantities $s_{m}$ and $s$ take on the values $x-x_{1}, y-y_{1}$ and $x-x_{2}, y-y_{2}$, respectively. Thus, to find the derivatives with respect to $\mathrm{x}, \mathrm{y}$ and $\mathrm{z}$, we need only compute the derivatives with respect to $\mathrm{s}, \mathrm{s}_{\mathrm{m}}$, and $\mathrm{z}$. Using the operator

$$
\nabla=\left(\partial / \partial s_{m}, \partial / \partial s, \partial / \partial z\right)
$$

we obtain

$$
\nabla w_{0}=\frac{\left[-\mathrm{ms}_{\mathrm{z}}, \mathrm{m}\left(\mathrm{x}_{\mathrm{m}} \mathrm{s}_{\mathrm{m}}-\mathrm{z}^{2}\right),-\mathrm{z}\left(\mathrm{s}_{\mathrm{m}}-\mathrm{ms}\right)\right]}{\mathrm{m}\left(\mathrm{z}_{\mathrm{m}}-\mathrm{z}^{2}\right) \mathrm{R}}
$$

Using the recursion relation (A9) to find $\mathrm{w}_{1}$ leads to

$$
\nabla w_{1}=\frac{m^{2}}{1-m^{2}}\left[\frac{\bar{R}_{1}}{R}-w_{0} \frac{\nabla x_{m}}{m}-x_{m} \frac{\nabla w_{0}}{m}\right]
$$

where $\quad \bar{R}_{1}=\left(s_{m},-s,-z\right)$ and $\quad \nabla x_{m}=(1,-1 / m, 0)$. 
Using the first of equations (A19), we find that

$$
\begin{aligned}
\nabla \mathrm{Q}_{1}= & \frac{\mathrm{x}_{\mathrm{m}}}{\left(\mathrm{s}_{\mathrm{m}}^{2}-\mathrm{s}^{2}\right)\left(\mathrm{z}_{\mathrm{m}}-\mathrm{z}^{2}\right)}\left\{( \mathrm { s } - \mathrm { s } _ { \mathrm { m } } / \mathrm { m } ) \left[\mathrm{R}\left(-\mathrm{z} / \mathrm{x}_{\mathrm{m}}, \mathrm{z} / \mathrm{mx} \mathrm{x}_{\mathrm{m}}, 1\right)\right.\right. \\
& \left.\left.\frac{-\mathrm{z}\left(\mathrm{s}_{\mathrm{m}},-\mathrm{s},-\mathrm{z}\right)}{\mathrm{R}}\right]-(1 / \mathrm{m},-1,0) \mathrm{zR}\right\}
\end{aligned}
$$

This formula holds for both $0<|\mathrm{m}|<1$ and $|\mathrm{m}|>1$.

The gradients of $R_{n}$ and $S_{n}$ can be found in terms of the other functions by differentiating under the integral sign, and in the case of $\mathrm{y}$, differentiating upper and lower limits as well. This yields

$$
\begin{aligned}
& \nabla R_{n}=\left(w_{m n}, R-w_{m n} / m,-z_{n}\right) \\
& \nabla S_{n}=\left(w_{n}, s^{n I_{0}}\left(r, s_{m}-r\right)-w_{n} / m,-z Q_{m n}\right)
\end{aligned}
$$

When $\mathrm{m}=0$, the panel edge is parallel to the panel $\mathrm{x}$ axis. If $\mathrm{m}=\infty$, the panel edge is parallel to the local $y$ axis. In order to represent all panel edge angles, for $|\mathrm{m}|<1$, for which the panel edge is subsonic, we introduce new variables

$$
\begin{aligned}
& \hat{x}_{\mathrm{m}}=\mathrm{mx}_{\mathrm{m}}=\mathrm{ms}_{\mathrm{m}}-\mathrm{s} \quad \hat{\mathrm{s}_{\mathrm{z}}}=\mathrm{ms}_{\mathrm{z}}=\mathrm{s}_{\mathrm{m}}-\mathrm{z}^{2} \\
& \hat{\mathrm{z}}_{\mathrm{m}}=\mathrm{m}^{2} \mathrm{z}_{\mathrm{m}}=\hat{\mathrm{x}}_{\mathrm{m}}^{2}+\mathrm{z}^{2}
\end{aligned}
$$

and define new $\mathrm{w}_{\mathrm{n}}$ function by

$$
\hat{w}_{\mathrm{n}}=\mathrm{w}_{\mathrm{n}} / \mathrm{m}
$$

For the supersonic panel edge $|\mathrm{m}|>1$ and we replace $1 / \mathrm{m}$ by $\lambda$ but retain the original form of the variables instead of the relations in equations (A25) and (A26). In this form the functions hold for all values of $\mathrm{m}, 0<|\mathrm{m}|<1$ and for all values of $\lambda, 0<|\lambda|<1$.

\section{A8. ANALYSIS OF SINGULARITIES OF THE FUNCTIONS FOR SUBINCLINED PANEL AND THEIR DERIVATIVES}

\section{A8.1 SINGULARITIES ON THE MACH CONE}

To find the effect on the flow pattern of discontinuities in doublet distribution and source distribution, it is essential to understand the singularities of the basic functions $w_{i}$ and $\mathrm{Q}_{\mathrm{I}}$ and their derivatives. From equation (A15) we see that for subsonic panel edges

$$
\mathrm{w}_{0} \sim \mathrm{R}
$$


near $\mathrm{R}=0$, the Mach cone through an endpoint of a panel edge. The gradient $\mathrm{W}_{0}$ then becomes infinite like $1 / \mathrm{R}$ as the field point approaches the Mach cone (see fig. 91). This is true for both subsonic and supersonic edges. When $|\mathrm{m}|>1$, the panel edge lies outside the Mach cone through the corner point, $w_{0}$ does not vanish on the Mach cone, however, but letting $R \rightarrow 0$ in equations (A16) yields

$$
\mathrm{w}_{0}= \pm \pi / 2 \sqrt{1-\lambda^{2}}
$$

Since $\mathrm{x}_{\mathrm{m}}$ is always positive downstream of the panel edge, the sign is according to the sign of $s-\lambda s_{m}$. Using the recursion rclation in cquation (A9), wc see that for subsonic panel edges

$$
\mathrm{w}_{1} \sim \mathrm{R}
$$

near the Mach cone. For the supersonic panel edge

$$
\mathrm{w}_{1}=\lambda \mathrm{x}_{\mathrm{m}} \mathrm{w}_{0} /\left(\lambda^{2}-1\right)= \pm \pi \lambda \mathrm{x}_{\mathrm{m}} / 2 \sqrt{\left(1-\lambda^{2}\right)^{3}}
$$

on the Mach cone. The quantities $\mathrm{w}_{0}$ and $\mathrm{w}_{1}$ are defined inside the region between the Mach cones from the two ends of the panel edge and Mach wedge formed by the planes containing the edge and tangent to the two end Mach cones (see fig. 92). This wedge region is defined by the relation

$$
z_{m}-z^{2} \geq 0
$$

In this region the value of $w_{0}$ and $w_{1}$ take the limiting value on the Mach cone given by equations (A27) and (A28).

On $\mathrm{z}=0, \mathrm{w}_{0}$ when evaluated for each end point is a function which is conical about the end point as illustrated in figure 91 . The combination of $w_{0}$ evaluated at the endpoints

$$
\mathrm{w}_{012}=\left(\mathrm{w}_{0}\right)_{1}-\left(\mathrm{w}_{0}\right)_{2}
$$

leads to the total contribution from the panel edge described in figure 92 .

The other function which is also defined in the Mach wedge is $Q_{I}$. The variable $x_{m}$ is zero on the vertex of the Mach wedge and is positive within it. Thus, it is seen that for $|\mathrm{m}|>1$,

$$
\mathrm{Q}_{\mathrm{I}}= \pm \pi / 2
$$

as $R \rightarrow 0$ with the sign determined by $z\left(s-\lambda s_{m}\right)$ while for subsonic panel edges, $Q_{I}$ goes to zero like $R \rightarrow 0$. Since $Q_{I} \sim R$, then gradient of $Q_{I}$ becomes infinite like $1 / R$ near the Mach cone. 
The value of $Q_{I}$ for $z \simeq 0$ in the Mach wedge is easily shown for $|m|>1$. We must consider the contribution from the endpoints of the panel edge:

$$
\mathrm{Q}_{11}-\mathrm{Q}_{12}
$$

Downstream of the panel edge $x_{m}>0$. The quantity $s-s_{m} / m$ is proportional to the oblique coordinate to $\mathrm{x}_{\mathrm{m}}$, say $\mathrm{y}_{\mathrm{m}}$.

For points $\mathrm{x}$, $\mathrm{y}$ lying between

$$
\mathrm{y}_{\mathrm{ml}}=\mathrm{s}_{1}-\mathrm{s}_{\mathrm{ml}} / \mathrm{m}=0
$$

and

$$
\mathrm{y}_{\mathrm{m} 2}=\mathrm{s}_{2}-\mathrm{s}_{\mathrm{m} 2} / \mathrm{m}=0
$$

the quantity $y_{m 2}>0$ and $y_{m 1}<0$. For this case $Q_{I 1}=-(\pi / 2) \operatorname{sign}(z)$ and $Q_{I 2}=(\pi / 2)$ sign $(z)$ and we have

$$
\mathrm{Q}_{\mathrm{I} 1}-\mathrm{Q}_{\mathrm{I} 2}=-\operatorname{sign}(\mathrm{z})
$$

For points outside for which $\mathrm{y}_{\mathrm{m} 1}$ and $\mathrm{y}_{\mathrm{m} 2}$ have the same sign and $\mathrm{R}=0$,

$$
\mathrm{Q}_{\mathrm{I} 1}-\mathrm{Q}_{\mathrm{I} 2}=0
$$

For subsonic leading edges and $z \rightarrow 0$, the function $Q_{I}= \pm \pi / 2$ if $R \neq 0$. The sign is determined by the sign of $\mathrm{z}$ and of $\mathrm{mx}_{\mathrm{m}}$. Consider a point downstream of the intersection of two subsonic panel edges as illustrated in figure 93 . The quantity $\mathrm{mx}_{\mathrm{m}}$ is positive for the right hand edge in figure 93 and negative for the left hand edge. Since the influence coefficient from a panel is found by going around the panel in the counterclockwise direction the value of $\mathrm{Q}_{\mathrm{I}}$ from equation (A19) for the two sides evaluated at the common endpoint for $|\mathrm{m}|<1$ is

$$
-\pi \operatorname{sign}(\mathrm{z})
$$

For points between the Mach lines and the nearby panel edge the contribution from the function $\mathrm{Q}_{\mathrm{I}}$ is zero. In the region between the Mach cones inside the Mach wedge for a supersonic panel edge equation (A30) holds. 


\section{A8.2 SINGULARITIES ON PANEL EDGES}

For $\mathrm{z}=0$, we have, for subsonic panel edges

$$
\mathrm{w}_{0}=\frac{\mathrm{m}}{2 \sqrt{1-\mathrm{m}^{2}}} \log \left[\frac{\left(\mathrm{s}_{\mathrm{m}}-\mathrm{ms}\right)+\sqrt{\left(1-\mathrm{m}^{2}\right)\left(\mathrm{s}^{2}-\mathrm{s}^{2}\right)}}{\left(\mathrm{s}_{\mathrm{m}}-\mathrm{ms}\right)-\sqrt{\left(1-\mathrm{m}^{2}\right)\left(\mathrm{s}^{2}-\mathrm{s}^{2}\right)}}\right]
$$

Upon multiplying both the numerator and denominator by the denominator, the relation takes the form

$$
\mathrm{w}_{0}=\frac{\mathrm{m}}{\sqrt{1-\mathrm{m}^{2}}} \log \left[\frac{\mathrm{m}\left(\mathrm{s}_{\mathrm{m}}-\mathrm{s} / \mathrm{m}\right)}{\left(\mathrm{s}_{\mathrm{m}}-\mathrm{ms}\right)-\sqrt{\left(1-\mathrm{m}^{2}\right)\left(\mathrm{s}_{\mathrm{m}^{2}-\mathrm{s}^{2}}\right)}}\right]
$$

Since $s_{m}-s / m=x_{m}=0$ is the panel edge, we see that $w_{0}$ has a logarithmic singularity at the panel edge. From equation $(\Lambda 21)$ setting $z=0$ yields

$$
\nabla w_{0}=\frac{\left(-s, s_{m}, 0\right)}{x_{m} \sqrt{s^{2} m-s^{2}}}
$$

The gradient of $\mathrm{w}_{0}$ has a higher order singularity $1 / \mathrm{x}_{\mathrm{m}}$ at the subsonic panel edge. Since this formula holds for supersonic edges as well, the gradient has a $1 / \sqrt{s_{m} \pm s}$ singularity on the two Mach lines through the corner point and is zero between the Mach lines and the panel edge. The gradient $\mathrm{Q}_{\mathrm{I}}$ on the panel is given by setting $\mathrm{z}=0$ in equation (A23), or

$$
\begin{aligned}
& \nabla Q_{I}=\frac{1}{x_{m}}\left\{\frac{\left(s-s_{m} / m\right)}{\left(s_{m}^{2}-s^{2}\right)} R(0,0,1)\right\} \\
& \partial Q_{I} / \partial z=\frac{\left(s-s_{m} / m\right)}{x_{m} \sqrt{s_{m}^{2}-s^{2}}}, \frac{\partial Q_{I}}{\partial x}=\frac{\partial Q_{I}}{\partial y}=0 .
\end{aligned}
$$

For $|\mathrm{m}|<1$, this has a $1 / \mathrm{x}_{\mathrm{m}}$ singularity near the panel edge $\mathrm{x}_{\mathrm{m}}=0$. For the supersonic panel edge $|\mathrm{m}|>1$, and $\partial \mathrm{Q}_{\mathrm{I}} / \partial \mathrm{z}$ is zero ahead of the Mach line. Also $\partial \mathrm{Q}_{\mathrm{I}} / \partial \mathrm{z}$ has a $1 / \sqrt{\mathrm{s}_{\mathrm{m}} \pm \mathrm{s}}$ singularity at Mach lines. When the panel edge is a Mach line, then the singularity is $1 / \sqrt{x_{m}}$ at the panel edge. 
A9. DERIVATION OF THE BASIC FUNCTIONS FOR SUPERINCLINED PANELS (SECTION 9)

We define the following functions:

$$
\widetilde{Q}_{I}=x_{e} \int \frac{d \zeta}{r^{2} R_{s}} \text { and } \tilde{w}_{0}=\int \frac{d \zeta}{R_{s}}
$$

where

$$
r^{2}=y_{e}^{2}+\left(\xi-z_{e}\right)^{2} \text { and } R_{s}=\sqrt{x^{2}-r^{2}}
$$

Let

$$
t=r^{2}=y_{e}^{2}+\left(\zeta-z_{e}\right)^{2} \text { or } \zeta-z_{e}=\sqrt{t-y_{e}^{2}} \text {. Then } d \zeta=d t / 2 \sqrt{t-y_{e}^{2}}
$$

and

$$
\widetilde{Q}_{I}=\frac{x y_{e}}{2} \int \frac{d t}{t \sqrt{\left(x^{2}-t\right)\left(t-y^{2}\right)}}
$$

Integration yields, after some manipulation,

$$
\tilde{\mathrm{Q}}_{\mathrm{I}}=\frac{1}{2} \tan ^{-1}\left[\frac{\mathrm{x}^{2}\left(\zeta-\mathrm{z}_{\mathrm{e}}\right)^{2}-\mathrm{y}_{\mathrm{e}}{ }^{2} \mathrm{R}_{\mathrm{s}}^{2}}{2 \mathrm{xy}_{\mathrm{e}}\left(\zeta-\mathrm{z}_{\mathrm{e}}\right) \mathrm{R}_{\mathrm{s}}}\right]
$$

Using the formula, $\tan ^{-1}(1 / \mathrm{X})=\pi / 2-\tan ^{-1}(\mathrm{X})$, yields

$$
\tilde{Q}_{I}=-\frac{1}{2} \tan ^{-1}\left[\frac{2 y_{e} R_{S} / x\left(\zeta-z_{e}\right)}{1-\frac{y^{2} R_{S}^{2}}{x^{2}\left(\zeta-z_{e}\right)^{2}}}\right]=\frac{1}{2} \tan ^{-1}\left[\frac{-2 y_{e} R / x(\zeta-z)}{1-\frac{y^{2} R_{s}^{2}}{x^{2}\left(\zeta-z_{e}\right)^{2}}}\right]
$$

This can be further simplified by applying the formulae

$$
\tan 2 \theta=\frac{2 \tan \theta}{1-\tan ^{2} \theta}
$$

and

$$
\tan ^{-1}(1 / x)=\pi / 2-\tan ^{-1}(\mathbf{x})
$$

Thus

$$
\widetilde{Q}_{I}=\tan ^{-1}\left[\frac{x\left(z_{e}-\zeta\right)}{-y_{e} R_{S}}\right]
$$

115 
The function $\widetilde{w}_{0}$ is seen to be

$$
\tilde{w}_{0}=\int \frac{\mathrm{d} \zeta}{\mathrm{R}_{\mathrm{s}}}=\int \frac{\mathrm{d} \zeta}{\sqrt{\mathrm{x}^{2}-\mathrm{y}_{\mathrm{e}}^{2}-\left(\zeta-\mathrm{z}_{\mathrm{e}}\right)^{2}}}=\tan ^{-1}\left(\frac{\zeta-\mathrm{z}_{\mathrm{e}}}{\mathrm{R}_{\mathrm{s}}}\right)
$$

Consider

The higher order functions $w_{n}=\int \frac{\zeta^{n} d \zeta}{R_{s}}$, can be evaluated by a recursion relation.

$$
\frac{d}{d \zeta}\left(\zeta^{n} R_{s}\right)=\frac{n \xi^{n-1} R_{s}^{2}-\zeta^{n}\left(\zeta-z_{e}\right)}{R_{s}}
$$

Integrating yields

$$
\widetilde{w}_{n+1}=\frac{1}{n+1}\left[(2 n+1) z_{e} \widetilde{w}_{n}+n\left(x^{2}-y_{e}^{2}-z_{e}^{2}\right) \widetilde{w}_{n-1}-\xi^{n} R_{s}\right]
$$

from which

$$
\begin{aligned}
& \widetilde{w}_{1}=z_{e} \widetilde{w}_{0}-R_{s} \\
& \widetilde{w}_{2}=\frac{1}{2}\left[3 z_{e} \widetilde{w}_{1}+\left(x^{2}-y_{e}{ }^{2}-z_{e} 2\right) \widetilde{w}_{0}-\zeta R_{s}\right]
\end{aligned}
$$


APPENDIX B:

DERIVATION OF THE INFLUENCE COEFFICIENTS FOR SUBINCLINED PANELS OF SMALL RELATIVE CURVATURE

B1. THE CURVED DOUBLET PANEL

\section{B1.1 EVALUATION OF THE FIRST INTEGRAL IN EQUATION (5.10) TO THE FIRST ORDER IN $\zeta$}

The first integral of equation (5.10) is expanded in the $\epsilon$ 's and only first order terms are retained. Using the expansions

$$
\begin{aligned}
& \mu\left(\mathrm{x}-\mathrm{r}+\epsilon_{1}-\mathrm{t}, \mathrm{y}_{0}\right)=\mu\left(\mathrm{x}-\mathrm{r}-\mathrm{t}, \mathrm{y}_{0}\right)+\epsilon_{1} \mu_{\mathrm{x}}\left(\mathrm{x}-\mathrm{v}-\mathrm{t}, \mathrm{y}_{0}\right) \\
& \frac{1}{\sqrt{\mathrm{t}\left(\mathrm{a}_{1} \mathrm{t}+\mathrm{b}_{1}\right)}}=\frac{1}{\sqrt{\mathrm{t}(\mathrm{t}+2 \mathrm{r})}}\left[1+\frac{2 \epsilon_{3}-\epsilon_{2} \mathrm{t}}{2(\mathrm{t}+2 \mathrm{r})}+\ldots\right] \\
& \int_{0}^{\mathrm{x}+\epsilon_{1}} \mathrm{~F}\left(\mathrm{x}_{0}\right) \mathrm{dx_{0 }}=\int_{0}^{\mathrm{x}} \mathrm{F}\left(\mathrm{x}_{0}\right) \mathrm{dx}_{0}+\epsilon_{1} \mathrm{~F}(\mathrm{x})
\end{aligned}
$$

and substituting the new variables

$$
\begin{aligned}
& x_{m}=x-x_{1}-\left(y-y_{1}\right) / m, s=y-y_{0} \\
& s_{m}=x-x_{1}+\left(y_{0}-y_{1}\right) / m=x_{m}+s / m \\
& r^{2}=s^{2}+z^{2}
\end{aligned}
$$

we oblain

$$
\begin{aligned}
-\frac{1}{2 \pi} \frac{\partial}{\partial z}\left\{\int_{\mathrm{y}-\mathrm{y}_{2}}^{\mathrm{y}-\mathrm{y} 1}\right. & \int_{0}^{\mathrm{s}_{\mathrm{m}}-\mathrm{r}} \frac{\mu(\mathrm{x}-\mathrm{r}-\mathrm{t}, \mathrm{y}-\mathrm{s})+(\mathrm{z} / \mathrm{r}) \zeta(\mathrm{x}-\mathrm{r}, \mathrm{y}-\mathrm{s}) \mu_{\mathrm{x}}(\mathrm{y}-\mathrm{r}-\mathrm{t}, \mathrm{y}-\mathrm{s}) \mathrm{dtds}}{\sqrt{\mathrm{t}(\mathrm{t}+2 \mathrm{r})}} \\
& +\mathrm{z} \int_{\mathrm{y}-\mathrm{y}_{2}}^{\mathrm{y}-\mathrm{y}_{1}} \frac{\mu\left(\mathrm{x}-\mathrm{s}_{\mathrm{m}}, \mathrm{y}-\mathrm{s}\right) \zeta(\mathrm{x}-\mathrm{r}, \mathrm{y}-\mathrm{s}) \mathrm{ds}}{\mathrm{r}^{2} \sqrt{\mathrm{s}_{\mathrm{m}^{2}-\mathrm{r}^{2}}}} \\
& -\frac{\mathrm{z}}{2} \int_{\mathrm{y}-\mathrm{y}_{2}}^{\mathrm{y}-\mathrm{y}_{1}} \int_{0}^{\mathrm{s}^{-r}} \frac{\mu(\mathrm{x}-\mathrm{r}-\mathrm{t}, \mathrm{y}-\mathrm{s})\left[\zeta_{\mathrm{xx}} \mathrm{t}-2\left(\bar{\xi}_{\mathrm{x}}+\xi / \mathrm{r}\right)\right] \mathrm{dtds}}{\sqrt{\mathrm{t}(\mathrm{t}+2 \mathrm{r})^{3}}}
\end{aligned}
$$


To simplify the last integral of equation (B2), we replace the first term in square brackets with

$$
\zeta_{x x} t /(t+2 r)=[1-2 r /(t+2 r)] \zeta_{x x}
$$

and expand the remaining terms to obtain

$$
\bar{\zeta}_{\mathrm{X}}+\bar{\zeta} / \mathrm{r}=\bar{\zeta}_{\mathrm{X}}(\mathrm{x}-\mathrm{r}, \mathrm{y}-\mathrm{s})+\zeta(\mathrm{x}-\mathrm{r}, \mathrm{y}-\mathrm{s}) / \mathrm{r}=\zeta_{0} / \mathrm{r}-\mathrm{r} \zeta_{\mathrm{xX}} / 2
$$

where $\zeta_{0}=\zeta(x, y-s)$.

Since

$$
\int \frac{d t}{\sqrt{t(t+2 r)^{3}}}=\int \frac{d(t+2 r)}{(t+2 r) \sqrt{(t+2 r)[(t+2 r)-2 r]}}=\frac{t}{r \sqrt{t(t+2 r)}}
$$

integrating the term

$$
\frac{2\left(\bar{\zeta}_{\mathrm{X}}+\bar{\zeta} / \mathrm{r}\right)}{\sqrt{\mathrm{t}(\mathrm{t}+2 \mathrm{r})^{3}}}
$$

of equation (B2) by parts and combining all similar terms in the resulting equation yields

$$
\begin{aligned}
& -\frac{1}{2 \pi} \int_{\frac{\partial}{\partial z}\left\{\left(1-z \zeta_{x x} / 2\right) \int_{y-y_{2}}^{y-y_{1}} \int_{0}^{s_{m}-r} \frac{\mu(x-r-t, y-s) d t d s}{\sqrt{t(t+2 r)}}\right.}^{+z \int_{y-y_{2}}^{y-y_{1}} \frac{\mu\left(x-s_{m}, y-s\right)\left[s_{m}\left(\zeta_{0} / r^{2}+\zeta_{x x} / 2\right)-\zeta_{0 x}\right] d s}{\sqrt{s^{2}-r^{2}}}} \\
& \left.+z \int_{y-y_{2}}^{y-y_{1}} \int_{0}^{\frac{s_{m}-r}{\sqrt{t(t+2 r)}}\left[\frac{\zeta(x-r, y-s)}{r}+t\left(\frac{\zeta_{0}}{r^{2}}+\frac{\zeta_{x x}}{2}\right)\right] d t d s}\right\}
\end{aligned}
$$


To perform the integrations with respect to $t$, we define the functions

$$
I_{n}(r, t)=\int_{0}^{t} \frac{t^{n} d t}{\sqrt{t(t+2 r)}}
$$

Expanding

$$
\mu(x-r-t, y-s)=\bar{\mu}-\bar{\mu}_{x} t+\mu_{x x} t^{2} / 2
$$

where $\bar{\mu}=\mu(\mathrm{x}-\mathrm{r}, \mathrm{y}-\mathrm{s})$

and

$$
\bar{\zeta}=\zeta(x-r, y-s)=\zeta_{0}-r \xi_{0 x}+r^{2} \xi x x / 2
$$

where $\zeta_{0}=\zeta(\mathrm{x}, \mathrm{y}-\mathrm{s})$

equation (B3) then becomes

$$
\begin{aligned}
& -\frac{1}{2 \pi} \frac{\partial}{\partial z}\left\{\left(1-z \zeta_{x x} / 2\right) \int_{y-y_{2}}^{y-y_{1}}\left[\bar{\mu} I_{0}-I_{1} \bar{\mu}_{x}+I_{2} \bar{\mu}_{x x} / 2\right] d s\right. \\
& +2 \int_{y-y_{2}}^{y-y_{1}} \frac{\mu\left(x-s_{m}, y-s\right)\left[s_{m}\left(\zeta_{0} / r^{2}+\zeta_{x x} / 2\right)-\zeta_{0 x}\right] d s}{\sqrt{s_{m}^{2}-r^{2}}} \\
& \int_{y-y_{2}}^{y-y}{ }_{-I_{1}\left(\mu_{x x} \zeta(x-r, y-s) / r-\bar{\mu}_{x}\left(\zeta_{0} / r^{2}+\zeta_{x x} / 2\right)\right)}^{r} \frac{\zeta(x-r, y-s)}{r} I_{0} \\
& \left.-I_{2} \mu_{x x}\left(\zeta_{0} / r^{2}+\zeta_{x x} / 2\right)\right] d s
\end{aligned}
$$


where $I_{n}=I_{n}\left(r, s_{m}-r\right)$.

The integrals in equation (B6) are further simplified by expanding

$$
\bar{\mu}=\mu(\mathrm{x}-\mathrm{r}, \mathrm{y}-\mathrm{s}) \text { and } \zeta(\mathrm{x}-\mathrm{r}, \mathrm{y}-\mathrm{s})
$$

in $\mathrm{r}$ and using the recursion relations in equation (A8), we obtain

$$
\begin{aligned}
& -\frac{1}{2 n} \frac{\partial}{\partial z}\left\{\left(1-z \zeta_{x x} / 2\right) \int_{y-y_{2}}^{y-y_{1}}\left[\left(\mu_{0}+r^{2} \zeta_{x x} / 4\right) I_{0}-\left(\mu_{0 x}-\frac{s_{m} \mu_{x x}}{4}\right) \sqrt{s_{m^{2}}-r^{2}}\right] d s\right. \\
& +z \int_{y-y_{2}}^{y-y_{1}} \mu\left(x-s_{m}, y-s\right)\left[s_{m}\left(\zeta_{0} / r^{2}+\zeta_{x x} / 2\right)-\zeta_{0 x}\right] d s / \sqrt{s_{m^{2}-r^{2}}} \\
& \int_{-z-y_{2}}^{y-y_{1}}\left[\zeta_{0 x} \mu_{0 x}+\left(\zeta_{0}+r^{2} \zeta_{x x} / 2\right) \mu_{x x} / 2\right] I_{0} d s \\
& +z \\
& \left.\int_{y-y_{2}}^{y-y_{1}}\left[\zeta_{0 x} \mu_{x x}+\left(\zeta_{0} / r^{2}+\zeta_{x x} / 2\right)\left(\mu_{0 x}-\frac{s_{m}}{2} \mu_{x x}\right)\right] \sqrt{s_{m^{2}-r^{2}}^{d s}}\right\}
\end{aligned}
$$

Since $\sqrt{s_{m}^{2}-r^{2}} / r^{2}=s_{m} 2 / r^{2} \sqrt{s_{m}^{2}-r^{2}}-1 / \sqrt{s_{m}^{2}-r^{2}}$

expanding $\mu\left(x-s_{m}, y-s\right)$ in powers of $s_{m}$ and collecting coefficients of

$$
\mu(\mathrm{x}, \mathrm{y}-\mathrm{s}), \mu_{\mathrm{x}}(\mathrm{x}, \mathrm{y}-\mathrm{s}), \mu_{\mathrm{xx}}
$$

yields

$$
\phi(z)=-\frac{1}{2 \pi} \frac{\partial}{\partial z} \int_{y-y_{2}}^{y-y}\left\{\mu(x, y-s) \psi_{1}(s)+\mu_{x}(x, y-s) \psi_{2}(s)+\mu_{x x} \psi_{3}(s)\right\} d s
$$


where

$$
\begin{aligned}
\psi_{1}= & \left(1-z \zeta_{x x} / 2\right) I_{0}+z\left[\frac{s_{m} \zeta_{0}}{r^{2} \sqrt{s_{m}^{2}-r^{2}}}+\frac{s_{m} \zeta_{x x} / 2-\zeta_{0 x}}{\sqrt{s_{m}^{2}-r^{2}}}\right] \\
\psi_{2}= & -z \zeta_{0 x} I_{0}+\left(z \zeta_{x x}-1\right) \sqrt{s_{m}^{2}-r^{2}}-\frac{z\left[\zeta_{0}-s_{m} \zeta_{0 x}+s_{m}^{2} \zeta_{x x} / 2\right]}{\sqrt{s_{m}^{2}-r^{2}}} \\
\psi_{3}= & {\left[\left(1-\frac{3 z \zeta_{x x}}{2}\right) \frac{r^{2}}{4}-\frac{z \zeta_{0}}{2}\right] I_{0}+\left[z \zeta_{0 x}+\left(1-\frac{3 z \zeta_{x x}}{2}\right) \frac{s_{m}}{4}\right] \sqrt{s_{m}^{2}-r^{2}} } \\
& +\frac{z s_{m}}{2}\left(\zeta_{0}-s_{m} \zeta_{0 x}+s_{m}^{2} \zeta_{x x} / 2\right) / \sqrt{s_{m}^{2}-r^{2}}
\end{aligned}
$$

Before performing the integration and differentiation, we now examine the other integrals and cast them in a similar form.

\section{B1.2 EVALUATION OF THE SECOND INTEGRAL OF EQUATION (5.10)}

Performing the differentiation with respect to $x$ of the second integral in equation $(5.10)$ and introducing the variables in equation (B1), yields

$$
\begin{aligned}
\phi(x)= & -\frac{1}{2 \pi} \int_{y-y_{2}}^{y-y_{1}} \frac{\zeta_{x}\left(x-s_{m}, y-s\right) \mu\left(x-s_{m}, y-s\right) d s}{\sqrt{s_{m}^{2}-r^{2}}} \\
& -\frac{1}{2 \pi} \int_{y-y_{2}}^{y-y_{1}} \int_{0}^{s_{m}-r}\left[\zeta_{x x^{\mu}}(x-r-t, y-s)\right. \\
& \left.+\zeta_{x}(x-r-t, y-s) \mu_{x}(x-r-t, y-s)\right] d t d s / \sqrt{t(t+2 r)}
\end{aligned}
$$


To perform the integration with respect to $t$, we expand the quantity in square brackets in powers of $t$. Integration of the second integral in equation (B10) with respect to t yields

$$
\begin{aligned}
& -\frac{1}{2 \pi} \int_{y-y_{2}}^{y-y_{1}}\left[\left(\zeta_{x x} \vec{\mu}+\zeta_{0 x} \bar{\mu}_{x}\right)_{0}^{I_{0}}\right. \\
& \left.-\left(2 \zeta_{x x} \mu_{x}+\zeta_{0 x} \mu_{x x}\right) I_{1}+3 \zeta_{x x} \mu_{x x} I_{2}\right] d s
\end{aligned}
$$

Applying the recursion relations in equation (A8) leads to

$$
\begin{gathered}
=-\frac{1}{2 \pi} \int_{y-y_{2}}^{y-y}\left\{\left[\left(\zeta_{x x} \bar{\mu}+\zeta_{0 x} \bar{\mu}_{x}\right)+r\left(2 \zeta_{x x} \bar{\mu}_{x}+\zeta_{0 x} \mu_{x x}\right)\right.\right. \\
\left.+9 r 2 \zeta_{x x} \mu_{x x} / 4\right] I_{0}-\left(2 \zeta_{x x} \bar{\mu}_{x}+\zeta_{0 x} \mu_{x x}\right) \sqrt{s_{m^{2}-r^{2}}} \\
\left.+\frac{3\left(s_{m}-4 r\right)}{4} \zeta_{x x} \mu_{x x} \sqrt{s_{m}^{2}-r^{2}}\right\} d s
\end{gathered}
$$

Equation (B10) is placed in the form of equation (B8) by expanding $\bar{\mu}$ in powers of $\mathrm{r}$ and $\mu\left(x-s_{m}, y-s\right)$ in powers of $s_{m}$. This yields finally for equation (B10), with equation (B11),

$$
\phi(\mathrm{x})=-\frac{1}{2 \pi} \int_{\mathrm{y}-\mathrm{y}_{2}}^{\mathrm{y}-\mathrm{y}_{1}}\left[\Omega_{1}(\mathrm{~s}) \mu(\mathrm{x}, \mathrm{y}-\mathrm{s})+\Omega_{2}(\mathrm{~s}) \mu_{\mathrm{x}}(\mathrm{x}, \mathrm{y}-\mathrm{s})+\Omega_{3}(\mathrm{~s}) \mu_{\mathrm{xx}}\right] \mathrm{ds}
$$


where

$$
\begin{aligned}
& \Omega_{1}=\frac{\zeta_{\mathrm{x}}\left(\mathrm{x}-\mathrm{s}_{\mathrm{m}}, \mathrm{y}-\mathrm{s}\right)}{\sqrt{\mathrm{s}_{\mathrm{m}}^{2}-\mathrm{r}^{2}}}+\zeta_{\mathrm{xx}} \mathrm{I}_{0} \\
& \Omega_{2}=-\frac{\mathrm{s}_{\mathrm{m}} \zeta_{\mathrm{x}}(\mathrm{x}-\mathrm{sm}, \mathrm{y}-\mathrm{s})}{\sqrt{\mathrm{s}_{\mathrm{m}}^{2}-\mathrm{r}^{2}}}-2 \zeta_{\mathrm{xx}} \sqrt{\mathrm{s}_{\mathrm{m}}^{2}-\mathrm{r}^{2}}+\zeta_{0 \mathrm{x}} \mathrm{I}_{0} \\
& \Omega_{3}=\frac{\mathrm{s}_{\mathrm{m}}^{2} \zeta_{\mathrm{x}}\left(\mathrm{x}-\mathrm{s}_{\mathrm{m}}, \mathrm{y}-\mathrm{s}\right)}{2 \sqrt{\mathrm{s}_{\mathrm{m}}^{2}-\mathrm{r}^{2}}}-\left(\zeta_{0 \mathrm{x}}-3 \zeta_{\mathrm{xx}} \mathrm{s}_{\mathrm{m}} / 4\right) \sqrt{\mathrm{s}_{\mathrm{m}}^{2}-\mathrm{r}^{2}}+3 \mathrm{r}^{2} \zeta_{\mathrm{xx}} \mathrm{I}_{0} / 4
\end{aligned}
$$

\section{B1.3 EVALUATION OF THE THIRD INTEGRAL IN EQUATION (5.10)}

Performing the differentiation with respect to $y$ in the third integral of equation (5.10) and introducing the variables of equation (B1), yield

$$
\begin{aligned}
\phi(y)= & -\frac{1}{2 \pi} \int_{y-y_{2}}^{y-y_{1}} \frac{s \xi_{y}\left(x-s_{m}, y-s\right) \mu(x-s m, y-s) d s}{r \sqrt{s_{m^{2}-r^{2}}}} \\
& -\frac{1}{2 \pi} \int_{y-y_{2}}^{y-y_{1}} \frac{s}{r} \int_{0}^{s_{m^{-r}}}\left[\frac{\zeta_{x y} \mu(x-r-t, y-s)+\zeta_{y}(x-r-t, y-s) \mu_{x}(x-r-t, y-s) d s}{\sqrt{t(t+2 r)}}\right] \\
& -\frac{1}{2 \pi} \int_{y-y_{2}}^{y-y_{1}} \frac{s}{r} \int_{0}^{s_{m^{-r}}} \frac{\zeta_{y}(x-r-t, y) \mu\left(x-r-t, y_{0}\right) d t d s}{\sqrt{t(t+2 r)^{3}}}
\end{aligned}
$$

Since

$$
\int \frac{d t}{\sqrt{t(t+2 r)^{3}}}=\frac{t}{r \sqrt{t(t+2 r)}}
$$


integration of the last integral by parts leads to

$$
\begin{aligned}
\phi(y)= & -\frac{1}{2 \pi} \int_{y-y_{2}}^{y-y_{1}} \frac{s s_{m}\left[\zeta_{y}\left(x-s_{m}, y-s\right) \mu\left(x-s_{m}, y-s\right)\right] d s}{r^{2} \sqrt{s_{m^{2}} r^{2}}} \\
& -\frac{1}{2 \pi} \int_{y-y_{2}}^{y-y_{1}} s \int_{0}^{s_{m^{-r}}}(r+t)\left[\zeta_{x y} \mu(x-r-t, y-s)\right. \\
& \left.+\zeta_{y}(x-r-t, y-s) \mu_{x}(x-r-t, y-s)\right] d t d s / r^{2} \sqrt{t(t+2 r)}
\end{aligned}
$$

To in tegrate the last term with respect to $t$, we expand $\mu(x-r-t, y-s)$ and $\zeta(x-r-t, y-s)$ in powers of $t$ and apply the recursion relations in equation (A8). After considerable simplification, equation (B14) then becomes

$$
\begin{aligned}
\phi(y)= & -\frac{1}{2 \pi} \int_{y-y_{2}}^{y-y_{1}} s_{m} \xi_{y}\left(x-s_{m}, y-s\right) \mu\left(x-s_{m}, y-s\right) d s / r^{2} \sqrt{s_{m}^{2}-r^{2}} \\
& -\frac{1}{2} \pi \int_{y-y_{2}}^{y-y} 1-\left(2 \zeta_{x y} \mu_{x}+\zeta_{y} \mu_{x x}\right) I_{0} s / 2+\zeta_{x y} \mu_{x x} s \sqrt{s_{m^{2}-r^{2}}} \\
& \left.+\left[\zeta_{x y^{\prime \prime}}+\zeta_{y}\left(x-s_{m}, y-s\right)\left(\mu_{x}-s_{m} \mu_{x x} / 2\right)\right] s \sqrt{s_{m}^{2}-r^{2} / r^{2}}\right\} d s
\end{aligned}
$$

Finally, $\phi(y)$ takes the form

$$
\phi(y)=-\frac{1}{2 \pi} \int_{y-y_{2}}^{y-y_{1}}\left[\Phi_{1}(s) \mu(x, y-s)+\Phi_{2}(s) \mu_{x}(x, y-s)+\Phi_{3}(s) \mu_{x x}\right] d s
$$


where

$$
\begin{aligned}
& \Phi_{1}=s\left(s_{m} \zeta_{y} / r^{2}-\zeta_{x y}\right) / \sqrt{s_{m}^{2}-r^{2}} \\
& \Phi_{2}=-\zeta_{x y} s I_{0}-s \zeta_{y}\left(x-s_{m}, y-s\right) / \sqrt{s_{m^{2}}-r^{2}} \\
& \Phi_{3}=-\zeta_{y} s_{0} / 2+\zeta_{x y} s \sqrt{s_{m}^{2}-r^{2}}+s_{m} \zeta_{y}\left(x-s_{m}, y-s\right) / 2 \sqrt{s_{m^{2}}-r^{2}}
\end{aligned}
$$

\section{B1:4 INTEGRATION OF THE COMPLETE POTENTIAL}

From the form of equations (B8), (B12), and (B15), we see that the velocity potential from the doublet distributions is

$$
\begin{aligned}
& \phi=\phi(\mathrm{z})+\phi(\mathrm{x})+\phi(\mathrm{y}) \\
& \phi=-\frac{1}{2 \pi} \int_{\mathrm{y}-\mathrm{y}_{2}}^{\mathrm{y}-\mathrm{y}_{1}}\left[\omega_{1}(\mathrm{~s}) \mu(\mathrm{x}, \mathrm{y}-\mathrm{s})+\omega_{2}(\mathrm{~s}) \mu_{\mathrm{x}}(\mathrm{x}, \mathrm{y}-\mathrm{s})+\omega_{3}(\mathrm{~s}) \mu_{\mathrm{xx}}\right] \mathrm{ds}
\end{aligned}
$$

where $\quad \omega_{\mathrm{i}}=\frac{\partial}{\partial \mathrm{z}} \quad \psi_{\mathrm{i}}(\mathrm{s}, \mathrm{z})+\Omega_{\mathrm{i}}(\mathrm{s})+\Phi_{\mathrm{i}}(\mathrm{s}) \quad \mathrm{i}=1,2,3$

Since $\mu$ is a quadratic distribution of the form

$$
\mu=a_{0}+a_{1} x+a_{2} y+a_{3} x y+a_{4} x^{2}+a_{5} y^{2}
$$

then $\phi$ may be expressed as

$$
\phi=\sum_{\mathrm{n}=0}^{5} \mathrm{a}_{\mathrm{n}} \phi_{\mathrm{n}}
$$

To separate $\phi$ into its components, we define

$$
\alpha_{i}=-\frac{1}{2 \pi} \int_{y-y_{2}}^{y-y_{1}} \omega_{1}(s) s^{i} d s
$$




$$
\begin{aligned}
& \gamma_{i}=-\frac{1}{2 \pi} \int_{y-y_{2}}^{y-y_{1}} \omega_{2}(s) s s^{i} d s \\
& x=-\frac{1}{2 \pi} \int_{y-y_{2}}^{y-y_{1}} \omega_{3}(s) d s
\end{aligned}
$$

Then the potentials $\phi_{\mathrm{n}}$ become

$$
\begin{aligned}
& \phi_{0}=\alpha_{0} \\
& \phi_{1}=\mathrm{x} \alpha_{0}+\gamma_{0} \\
& \phi_{2}=\mathrm{y} \alpha_{0}-\alpha_{1} \\
& \phi_{3}=\mathrm{xy} \alpha_{0}-\mathrm{x} \alpha_{1}+\mathrm{y} \gamma_{0}-\gamma_{1} \\
& \phi_{4}=\mathrm{x}^{2} \alpha_{0}+\left(2 \mathrm{x}-\mathrm{x}_{\mathrm{m}}\right) \gamma_{0}-\gamma_{1} / \mathrm{m}+x \\
& \phi_{5}=\mathrm{y}^{2} \alpha_{0}-2 \mathrm{y} \alpha_{1}+\alpha_{2}
\end{aligned}
$$

The basic integrals needed in evaluating $\alpha_{\mathrm{i}}, \gamma_{\mathrm{i}}$ and $\mathrm{x}$

$$
\begin{aligned}
& R_{i}=\int s^{i} \sqrt{s_{m^{2}}-r^{2}} d s \\
& S_{i}=\int s^{i} I_{0}\left(r, s_{m}-r\right) d s \\
& Q_{i}=\int s^{i} d s / r^{2} \sqrt{s_{m^{2}-r^{2}}} \\
& w_{i}=\int s^{i} d s / \sqrt{s_{m^{2}}-r^{2}}
\end{aligned}
$$


The functions $R_{i}, S_{i}, Q_{i}$, and $W_{i}$ are derived in Appendix $A$. It is also convenient to use the subscript $m$ to define functions like

$$
w_{m i}=\int \frac{s_{m} s^{i} d s}{\sqrt{s_{m}^{2}-r^{2}}}=\int \frac{s^{i}\left(x_{m}+s / m\right)}{\sqrt{s_{m}^{2}-r^{2}}}=x_{m} w_{i}+w_{i+1} / m
$$

Similar notation is applied to the functions $S, Q$, and $R$ when required.

Using equations (B9), (B13), and (B16), we obtain for equations (B20), (B21), (B22)

$$
\begin{aligned}
& \alpha_{\mathrm{i}}=\frac{1}{2 \pi}\left\{-\frac{\partial}{\partial \mathrm{z}}\left[\left(1-\mathrm{z} \xi_{\mathrm{XX}} / 2\right) \mathrm{S}_{\mathrm{i}}+\mathrm{z}\left(\mathrm{A} \cdot \mathrm{Q}_{\mathrm{mi}}+\zeta_{\mathrm{Xx}} \mathrm{w}_{\mathrm{mi}} / 2-\mathrm{B} \cdot \mathrm{w}_{\mathrm{i}}\right)\right]\right. \\
& \left.-\zeta_{x x}\left(s_{i}-w_{m i}\right)-B \cdot w_{i}-\zeta_{y} Q_{m i+1}+\zeta_{y y} Q_{m i+2}+\zeta_{x y} w_{i+1}\right\} \\
& \gamma_{i}=-\frac{1}{2 \pi}\left\{\frac { \partial } { \partial z } \left[-z B \cdot S_{i}+\left(z \zeta_{x x^{-1}}\right) R_{j}-z\left(A \cdot w_{i}-B \cdot w_{m i}\right.\right.\right. \\
& \left.\left.+\zeta_{x x} w_{m m i} / 2\right)\right]+B \cdot S_{i}-B \cdot w_{m i}+\zeta_{x x} w_{m m i}-2 \xi_{x x} R_{i} \\
& \left.-\zeta_{x y} S_{i+1}-\zeta_{y} w_{i+1}+\zeta_{x y} w_{m i+1}+\zeta_{y y} w_{i+2}\right\} \\
& \chi=-\frac{1}{2 \pi}\left\{\frac { \partial } { \partial z } \left[\left(1-3 \frac{z \xi_{x x}}{2}\right)\left(z^{2} S_{0}+S_{2}\right) / 2-z A \cdot S_{0}-z B \cdot S_{m 0}\right.\right. \\
& \left.+2 z B R_{0}-\frac{1}{2}\left(1-\frac{z \zeta_{x x}}{2}\right) R_{m 0}\right]+B \cdot S_{m 0}-2 B \cdot R_{0}-\zeta_{x x} R_{m 0} / 2 \\
& \left.+\frac{3}{2} \zeta_{x x}\left(z^{2} s_{m 0}+s_{m 2}\right)-\zeta_{x y} s_{m 1}-\zeta_{y} s_{1}+\zeta_{y y} s_{2}+2 \zeta_{x y} R_{1}\right\}
\end{aligned}
$$


where the terms of the form $A \cdot w_{i}$ and $B \cdot w_{i}$ are defined by

$$
\begin{aligned}
& A \cdot w_{i}=\zeta w_{i}-\zeta_{y} w_{i+1}+\zeta_{y y} w_{i+2} / 2 \\
& B \cdot w_{i}=\zeta_{x} w_{i}-\zeta_{x y} w_{i+1}
\end{aligned}
$$

Since the integrands of $S_{i}$ and $R_{i}$ are continuous, differentiating under the integral sign is permissible and we obtain

$$
\frac{\partial S_{i}}{\partial z}=-z Q_{m i}, \frac{\partial R_{i}}{\partial z}=-z w_{i}
$$

With these relations, performing the differentiation with respect to $z$ of the terms indicated and simplifying leads to

$$
\begin{aligned}
& \alpha_{i}=\frac{1}{2 \pi}\left\{z Q_{m i}-\zeta_{x x}\left(S_{i}+z^{2} Q_{m i}\right) / 2\right. \\
& -\frac{\partial}{\partial z}\left(A \cdot Q_{m i} z\right)-z \frac{\partial}{\partial z}\left(\zeta_{x x} w_{m i} / 2-B_{i} w_{i}\right) \\
& +\zeta_{\mathrm{xx}} \mathrm{w}_{\mathrm{mi}} / 2-\zeta_{\mathrm{y}} \mathrm{Q}_{\mathrm{mi}}+1 \\
& \left.+\zeta_{y y} Q_{m i}+2+\zeta_{x y} w_{i+1}\right\} \\
& \gamma_{i}=\frac{1}{2 \pi}\left\{-z_{i}+\zeta_{x x}\left(z^{2} w_{i}+R_{i}\right)+B \cdot\left(w_{m i}-z^{2} Q_{m i}\right)\right. \\
& +\left(1+\mathrm{z} \frac{\partial}{\partial \mathrm{z}}\right)\left[\mathrm{A} \cdot \mathrm{w}_{\mathrm{i}}-\mathrm{B} \cdot \mathrm{w}_{\mathrm{mi}}+\zeta_{\mathrm{xx}} \mathrm{w}_{\mathrm{mmi}} / 2\right]+\zeta_{\mathrm{xx}} \mathrm{w}_{\mathrm{mmi}} \\
& \left.+\zeta_{x y} s_{i+1}+\zeta_{y} w_{i+1}-\zeta_{x y} w_{m i+1}-\zeta_{y y} w_{i}+2\right\} \\
& \chi=-\frac{1}{2 \pi}\left\{z_{0}+\left(\zeta_{x x} / 2\right)\left[z^{2} w_{m 0}-3 z^{2} S_{0} / 2+3 S_{2} / 2-R_{m 0} / 2\right]+z^{2} B \cdot\left(Q_{m m 0}-2 w_{0}\right)\right. \\
& \left.-\xi_{x y} S_{m 1}-\xi_{y} s_{1}+\xi_{y y} S_{2}+2 \zeta_{x y} R_{1}+A \cdot\left(z^{2} Q_{m 0}-S_{0}\right)\right\}
\end{aligned}
$$


When curvature is neglected, $\zeta=\mathrm{A} \cdot=\mathrm{B} \cdot=0$ and

$$
\begin{aligned}
& \alpha_{\mathrm{i}}=\mathrm{zQ}_{\mathrm{mi}} / 2 \pi \\
& \gamma_{\mathrm{i}}=-\mathrm{zw}_{\mathrm{i}} / 2 \pi \\
& \chi=-\mathrm{zS}_{0} / 2 \pi
\end{aligned}
$$

the potential then reduces to equation (5.17).

By examining the terms in the velocity potential involving $\zeta$, we find that the potential has the same singularities as the velocity components for the flat panel. From equation (B35) for $\alpha_{0}$, we find the term

$$
\frac{\partial}{\partial z}\left(A \cdot z Q_{m 0}\right)=\frac{\partial}{\partial z}\left(\zeta Q_{I}-z Q_{m 1} \xi_{y}+Q_{m} 2 \xi_{y y} / 2\right)
$$

leading to the derivative

$$
\partial \mathrm{Q}_{\mathrm{I}} / \partial \mathrm{z}
$$

From equation (A23) we see that the gradient of $\mathrm{Q}_{\mathrm{I}}$ has a $1 / \mathrm{R}$ singularity on the Mach cone $\mathrm{R}=0$. The velocity components then will have $1 / \mathrm{R}^{3}$ singularities on the Mach cone. This singularity is too strong since it is not integrable and renders the terms involving $\zeta$ impractical. Expanding the potential to the first order in $\zeta$ acts like a differentiation of the flat plate potential. To eliminate this singularity, the doublet distribution would have to be prescribed on the curved surface itself. It was decided that flat panels only would be used.

\section{B2. THE CURVED SOURCE PANEL}

We shall evaluate the influence coefficients for the source panel in a manner similar to that followed for the first integral of the doublet panel in the preceding section of B1.1. Equation (8.2) differs from the first integral of equation (5.10) only in the differentiation with respect to $\mathrm{z}$. Consequently, expanding this integral, retaining only first order terms in $\zeta$ can be written down from equation (B3). This is

$$
\begin{aligned}
\phi=-\frac{1}{2 \pi}\left\{\left(1-z \zeta_{\mathrm{xx}} / 2\right) \int_{\mathrm{y}-\mathrm{y}_{2}}^{\mathrm{y}-\mathrm{y} 1} \int_{0}^{\mathrm{s}^{-r}} \frac{\sigma(\mathrm{x}-\mathrm{r}-\mathrm{t}, \mathrm{y}-\mathrm{s}) \mathrm{dtds}}{\sqrt{\mathrm{t}(\mathrm{t}+2 \mathrm{r})}}\right. \\
+\mathrm{z} \int_{\mathrm{y}-\mathrm{y}_{2}}^{\mathrm{y}-\mathrm{y} 1} \frac{\sigma\left(\mathrm{x}-\mathrm{s}_{\mathrm{m}}, \mathrm{y}-\mathrm{s}\right)\left[\mathrm{s}_{\mathrm{m}}\left(\zeta_{0} / \mathrm{r}^{2}+\zeta_{\mathrm{xx}} / 2\right)-\zeta_{\mathrm{Ox}}\right] \mathrm{ds}}{\sqrt{\mathrm{s}^{2}-\mathrm{r}^{2}}}
\end{aligned}
$$




$$
\left.+z \int_{y-y_{2}}^{y-y_{1}} \int_{0}^{s_{m}-r} \frac{\sigma_{x}\left[\zeta(x-r, y-s) / r+t\left(\zeta_{0} / r^{2}+\zeta_{x x} / 2\right)\right] d t d s}{\sqrt{t(t+2 r)}}\right\}
$$

where $\zeta_{0}=\zeta(\mathrm{x}, \mathrm{y}-\mathrm{s})$ and $\mathrm{s}_{\mathrm{m}}$ and $\mathrm{r}$ are defined in equations (A3). Now

$$
\sigma(\mathrm{x}-\mathrm{r}-\mathrm{t}, \mathrm{y}-\mathrm{s})=\sigma(\mathrm{x}-\mathrm{r}, \mathrm{y}-\mathrm{s})-\sigma_{\mathrm{x}} \mathrm{t}
$$

since $\sigma$ is linear in $\mathrm{x}$ and $\mathrm{y}$. Performing the integration with respect to $\mathrm{t} y$ ields

$$
\begin{aligned}
\phi= & -\frac{1}{2 \pi} \int_{\mathrm{y}-\mathrm{y}_{2}}^{\mathrm{y}-\mathrm{y} 1}\left\{( 1 - \mathrm { z } \zeta _ { \mathrm { xx } } / 2 ) \left[\sigma(\mathrm{x}-\mathrm{r}, \mathrm{y}-\mathrm{s}) \mathrm{I}_{0}\left(\mathrm{r}, \mathrm{s}_{\mathrm{m}}-\mathrm{r}\right)\right.\right. \\
& \left.-\sigma_{\mathrm{X}} \mathrm{I}_{1}\left(\mathrm{r}, \mathrm{s}_{\mathrm{m}}-\mathrm{r}\right)\right]+\mathrm{z} \frac{\sigma\left(\mathrm{x}-\mathrm{s}_{\mathrm{m}}, \mathrm{y}-\mathrm{s}\right)\left[\mathrm{s}_{\mathrm{m}}\left(\zeta_{0} / \mathrm{r}^{2}+\zeta_{\mathrm{xx}} / 2\right)-\zeta_{0 \mathrm{x}}\right]}{\sqrt{\mathrm{s}_{\mathrm{m}}{ }^{2} \mathrm{r}^{2}}} \\
& \left.+\mathrm{z} \sigma_{\mathrm{X}}\left[\frac{\zeta(\mathrm{x}-\mathrm{r}, \mathrm{y}-\mathrm{s})}{\mathrm{r}} \mathrm{I}_{0}\left(\mathrm{r}, \mathrm{s}_{\mathrm{m}}-\mathrm{r}\right)+\left(\frac{\zeta_{0}}{\mathrm{r}^{2}}+\frac{\zeta_{\mathrm{Xx}}}{2}\right) \mathrm{I}_{1}\left(\mathrm{r}, \mathrm{s}_{\mathrm{m}}-\mathrm{r}\right)\right]\right\} \mathrm{d}
\end{aligned}
$$

Eliminating $I_{1}$ by means of equation (A8) and expanding

$$
\sigma(\mathrm{x}-\mathrm{r}, \mathrm{y}-\mathrm{s})=\sigma(\mathrm{x}, \mathrm{y}-\mathrm{s})-\mathrm{r} \sigma_{\mathrm{X}}=\sigma_{0}-\mathrm{r} \sigma_{\mathrm{X}}
$$

lead to

$$
\begin{aligned}
\phi= & -\frac{1}{2 \pi} \int_{\mathrm{y}-\mathrm{y}}^{2}{ }^{\mathrm{y}-\mathrm{y}} 1\left(1-\mathrm{z} \zeta_{\mathrm{xx}} / 2\right)\left[\sigma_{0} \mathrm{I}_{0}-\sigma_{\mathrm{x}} \mathrm{R}\right] \\
& +\mathrm{z} \sigma\left(\mathrm{x}-\mathrm{s}_{\mathrm{m}}, \mathrm{y}-\mathrm{s}\right)\left[\mathrm{s}_{\mathrm{m}}\left(\zeta_{0} / \mathrm{r}^{2}+\zeta_{\mathrm{xx}} / 2\right)-\zeta_{0 \mathrm{x}}\right] / \mathrm{R} \\
& \left.+\mathrm{z} \sigma_{\mathrm{x}}\left[-\zeta_{0 \mathrm{x}} \mathrm{I}_{0}+\left(\frac{\zeta_{0}}{\mathrm{r}^{2}}+\frac{\zeta_{\mathrm{xx}}}{2}\right) \mathrm{R}\right]\right\} \mathrm{ds}
\end{aligned}
$$

where

$$
R=\sqrt{s_{m}^{2}-r^{2}}
$$


To express the integrals in terms of the basic functions of appendix A, we substitute

$$
\frac{\mathrm{R}}{\mathrm{r}^{2}}=\frac{\mathrm{s}_{\mathrm{m}}^{2}}{\mathrm{r}^{2} \mathrm{R}}-\frac{1}{\mathrm{R}}
$$

We then expand $\sigma(\mathrm{x}, \mathrm{y}-\mathrm{s})$ and $\sigma\left(\mathrm{x}-\mathrm{s}_{\mathrm{m}}, \mathrm{y}-\mathrm{s}\right)$ in $\mathrm{s}$ and $\mathrm{s}_{\mathrm{m}}$. After combining and simplifying we obtain

$$
\phi=-\frac{1}{2 \pi} \int_{\mathrm{y}-\mathrm{y}_{1}}^{\mathrm{y}-\mathrm{y}_{1}}\left[\sigma(\mathrm{x}, \mathrm{y}) \psi_{1}(\mathrm{~s})+\sigma_{\mathrm{x}} \psi_{2}(\mathrm{~s})+\sigma_{\mathrm{y}} \psi_{3}(\mathrm{~s})\right] \mathrm{ds}
$$

where

$$
\begin{aligned}
& \psi_{1}=\left(1-z \zeta_{\mathrm{xx}} / 2\right) \mathrm{I}_{0}+\mathrm{zs} \mathrm{s}_{0} \zeta_{0} / \mathrm{r}^{2} \mathrm{R}+\mathrm{z}\left(\mathrm{s}_{\mathrm{m}} \zeta_{\mathrm{xx}} / 2-\zeta_{0 \mathrm{x}}\right) / \mathrm{R} \\
& \psi_{2}=-\mathrm{R}+\frac{\mathrm{z} \zeta_{\mathrm{Xx}}}{2 \mathrm{R}} \mathrm{s}_{\mathrm{m}}{ }^{2}+\frac{\mathrm{zs_{ \textrm {m } }}}{\mathrm{R}} \zeta_{0 \mathrm{x}}-\frac{\mathrm{z}}{\mathrm{R}} \zeta_{0} \\
& \psi_{3}=-\mathrm{s}\left(1-\mathrm{z} \zeta_{\mathrm{xx}} / 2\right) \mathrm{I}_{0}-\mathrm{z} \mathrm{s}_{\mathrm{m}} \zeta_{0} \mathrm{~s} / \mathrm{r}^{2} \mathrm{R}-\mathrm{zs}\left(\mathrm{s}_{\mathrm{m}} \zeta_{\mathrm{xx}} / 2-\zeta_{0 \mathrm{x}}\right) / \mathrm{R}
\end{aligned}
$$

where $\zeta_{0}=\zeta(\mathrm{x}, \mathrm{y}-\mathrm{s})$

Since the source distribution is linear in $\mathrm{x}$ and $\mathrm{y}$

$$
\sigma=a_{0}+a_{1} x+a_{2} y
$$

then the velocity potential may be expressed in the form

$$
\phi=\mathrm{a}_{0} \phi_{0}+\mathrm{a}_{1} \phi_{1}+\mathrm{a}_{2} \phi_{2}
$$

Then

$$
\phi_{0}=-\frac{1}{2 \pi} \int_{y-y_{2}}^{y-y_{1}} \psi_{1}(s) d s
$$




$$
=-\frac{1}{2 \pi} \int_{y-y_{2}}^{\mathrm{y}-\mathrm{y}_{1}}\left\{\left(1-z \zeta_{\mathrm{xx}} / 2\right) \mathrm{I}_{0}+\mathrm{zs}_{\mathrm{m}} \zeta_{0} / \mathrm{r}^{2} \mathrm{R}+\mathrm{z}\left(\zeta_{\mathrm{xx}} \mathrm{s}_{\mathrm{m}} / 2-\zeta_{0 \mathrm{x}}\right) / \mathrm{R}\right\} \mathrm{ds}
$$

and integrating yields

$$
\phi_{0}=\frac{1}{2 \pi}\left[S_{0}+z_{0}\right]
$$

where

$$
\chi_{0}=A \cdot Q_{m 0}+\zeta_{x x} w_{m 0} / 2-B \cdot w_{0}-\zeta_{x x} S_{0} / 2
$$

and $A \cdot$ and $B \cdot$ are defined in equations (B33) and (B34). Now

$$
\phi_{1}=\mathrm{x} \phi_{0}-\frac{1}{2 \pi} \int_{\mathrm{y}-\mathrm{y}_{2}}^{\mathrm{y}-\mathrm{y} 1} \psi_{2}(\mathrm{~s}) \mathrm{ds}
$$

Finally integration yields

$$
\phi_{1}=\mathrm{x} \phi_{0}+\mathrm{R}_{0} / 2 \pi+\mathrm{z}_{1} / 2 \pi
$$

where

$$
\chi_{1}=\mathrm{A} \cdot \mathrm{w}_{0}-\mathrm{B} \cdot \mathrm{w}_{\mathrm{m} 0}-\zeta_{\mathrm{xx}} \mathrm{w}_{\mathrm{mm} 0} / 2
$$

Similarly,

$$
\phi_{2}=\mathrm{y} \phi_{0}-\frac{1}{2 \pi} \int_{\mathrm{y}-\mathrm{y}_{2}}^{\mathrm{y}-\mathrm{y} 1} \psi_{3}(\mathrm{~s}) \mathrm{ds}
$$


and

$$
\phi_{2}=\mathrm{y} \phi_{0}+\mathrm{S}_{1} / 2 \pi+\mathrm{zx}_{2} / 2 \pi
$$

where

$$
\chi_{2}=\zeta_{x x}\left(w_{m 1}-S_{1}\right) / 2+A \cdot Q_{m 1}-B \cdot w_{1}
$$

To compute the perturbation velocity vector, it is convenient to write the complete velocity potential which becomes

$$
\phi=-\frac{1}{2 \pi}\left\{\sigma(\mathrm{x}, \mathrm{y})\left(\mathrm{S}_{0}+\mathrm{z}_{0}\right)-\sigma_{\mathrm{x}}\left(\mathrm{R}_{0}+\mathrm{z}_{1}\right)-\sigma_{\mathrm{y}}\left(\mathrm{S}_{1}+\mathrm{z}_{2}\right)\right\}
$$

In the same manner as for the doublet panel method, we obtain the relations for the velocity by the gradient operator

$$
\nabla=\left(\frac{\partial}{\partial s_{m}}, \frac{\partial}{\partial s}, \frac{\partial}{\partial z}\right)
$$

Thus, we obtain

$$
\begin{aligned}
\nabla \phi= & -\frac{1}{2 \pi}\left\{\sigma(\mathrm{x}, \mathrm{y})\left[\nabla \mathrm{S}_{0}+\nabla\left(\mathrm{z} \chi_{0}\right)\right]\right. \\
& +\left(\sigma_{\mathrm{x}}, \sigma_{\mathrm{y}}, 0\right)\left(\mathrm{S}_{0}+\mathrm{z} \chi_{0} / 2 \pi\right) \\
& -\sigma_{\mathrm{x}}\left[\nabla \mathrm{R}_{0}+\nabla\left(\mathrm{z} \mathrm{x}_{1}\right)\right] \\
& \left.-\sigma_{\mathrm{y}}\left[\nabla \mathrm{S}_{1}+\nabla\left(\mathrm{z} \chi_{2}\right)\right]\right\}
\end{aligned}
$$

Since the expression for the velocity potential contains the term

$$
\mathrm{zQ}_{\mathrm{mO}} \zeta
$$

in $z_{0}$ of equations (B53) and (B48), we see that the contribution to the source velocity components from the panel shape parameters have some of the same singularities as the flat doublet panel. As we discovered from the doublet, expanding in the shape parameter $\zeta$ acts as a differentiation of the basic flat panel aerodynamic inifuence coefficients. 


\section{APPENDIX C: \\ DESCRIPTION OF THE SPLINES FOR SUPERSONIC SOURCE AND DOUBLET PANELS}

\section{C1. SOURCE PANEL}

For each panel, an average plane is defined which passes through the panel center and midpoints of the lines joining the panel corners. On the panel projected onto this average plane, the source strength $\sigma$ is taken to be linear in the coordinates of this plane, i.e.,

$$
\sigma=\sigma_{0}+\sigma_{\mathrm{x}} \mathrm{x}+\sigma_{\mathrm{y}} \mathrm{y}
$$

The three parameters $\sigma_{\mathrm{O}}, \sigma_{\mathrm{x}}$, and $\sigma_{\mathrm{y}}$ which are needed to specify the source strength on each panel are not, per se, basic unknowns of the problem. Rather, they are calculated as needed in terms of the source strengths at the centers of the panels, which, in turn, are determined so that the solution meets the specified boundary conditions.

The parameters of equation (C1) are determined by a weighted least-squares fit to the source strength at the centers of the eight surrounding panels. That is, if the source strength at the center $\zeta_{\mathrm{i}}, \eta_{\mathrm{i}}$ of the ith of the 9 panels involved is called $\sigma_{\mathrm{i}}$ the quantity

$$
E=\sum_{i=1}^{9} w_{i}\left(\sigma_{i}-\sigma_{0}-\sigma_{x} \xi_{i}-\sigma_{y} \eta_{i}\right)^{2}
$$

is made stationary with respect to variations in $\sigma_{\mathrm{O}}, \sigma_{\mathrm{X}}$, and $\sigma_{\mathrm{y}}$. The weight $\mathrm{w}_{\mathrm{i}}$ is equal to $10^{8}$ if $i$ is the label of the center of the panel for which equation (C1) is sought, and $w_{i}=1$ otherwise.

If the panel is at the corner of the network, there are three neighboring panels, which is enough to make this system determinate (see figure 94).

Two points are worth noting about this representation of the source strength. First, there is little reason for the source strength to be continuous from one panel to the next, since source discontinuities do not introduce infinite singularities in the flow as doublet strength discontinuities do. Of course, in the limit as the grid is refined, we expect the discontinuitics in the source strength to vanish, so that these discontinuities furnish a measure of whether the mesh is sufficiently fine. Secondly, since the source distribution within the panels is determined in terms of the source strength at the centers of nearby panels, the number of unknowns governing the source strength is exactly equal to the number of panels. 


\section{C2. DOUBLET PANEL}

Since it was shown in section 3.7 that the jump in tangential velocity across a singularity distribution is related to the local value of the derivatives of the doublet strength, while the jump in normal flux is given in terms of the source strength, it is necessary to use a higher order representation of the doublet strength than of the source strength to achieve the same level of representation of their velocity fields. Thus, the doublet strength is represented by a quadratic:

$$
\mu=\mu_{\mathrm{o}}+\mu_{\mathrm{x}} \mathrm{x}+\mu_{\mathrm{y}} \mathrm{y}+\mu_{\mathrm{xx}} \mathrm{x}^{2} / 2+\mu_{\mathrm{xy}} \mathrm{xy}+\mu_{\mathrm{yy}} \mathrm{y}^{2} / 2
$$

For the six parametcr spline, a quantity similar to equation (C2) is formed for the doublet strength in equation (C3), namely,

$$
\mathrm{E}=\sum_{\mathrm{k}=1}^{\mathrm{N}} \mathrm{w}_{\mathrm{k}}\left(\mu_{0}+\mu_{\mathrm{x}} \xi_{\mathrm{k}}+\mu_{\mathrm{y}} \eta_{\mathrm{k}}+\mu_{\mathrm{xx}} \xi_{\mathrm{k}} 2 / 2+\mu_{\mathrm{xy}} \xi_{\mathrm{k}} \eta_{\mathrm{k}}+\mu_{\mathrm{y} y} \eta_{\mathrm{k}} 2 / 2-\lambda_{\mathrm{k}}\right)^{2}
$$

where $\lambda_{\mathrm{k}}$ is the value of the doublet strength at $\zeta_{\mathrm{k}}, \eta_{\mathrm{k}}$, the control point of the kth neighboring panel (or point on network edge) projected upon the average plane of the panel for which the coefficients are to be defined. The quantity $\mathrm{E}$ is made stationary with respect to the coefficients of the quadratic defining the doublet strength. To facilitate the determination of these coefficients, we define the vectors:

$$
\begin{gathered}
c_{i}=\left(\mu_{0}, \mu_{x}, \mu_{y}, \mu_{x x}, \mu_{x y}, \mu_{y y}\right), i=1,2,3, \ldots, 6 \\
a_{k i}=\left(1, \xi_{\mathrm{k}}, \eta_{\mathrm{k}}, \xi_{\mathrm{k}} 2 / 2, \xi_{\mathrm{k}} \eta_{\mathrm{k}}, \eta_{\mathrm{k}} 2 / 2\right) \begin{array}{l}
\mathrm{k} \text { not summed } \\
\mathrm{i}=1,2, \ldots, 6
\end{array}
\end{gathered}
$$

then

$$
E=\sum_{k=1}^{N}\left(c_{i} a_{k i}-\lambda_{k}\right)^{2} w_{k}
$$

from which

$$
\frac{\partial E}{\partial-j}=\sum_{k=1}^{\eta} w_{k}\left(c_{i} a_{k i}-\lambda_{k}\right) a_{j k}=0
$$


and

$$
\left\{\sum_{k=1}^{N} \quad a_{k j} \quad a_{k i} w_{k}\right\} c_{i}=\sum_{k=1}^{N} w_{k} \lambda_{k} a_{j k}
$$

Solving for $c_{i}$ yields

$$
c_{i}=\left\{\sum_{k=1}^{N} a_{k j} a_{k i} w_{k}\right\}^{-1}\left\{\sum_{k=1}^{N} w_{k} \lambda_{k} a_{j k}\right\}
$$

Equation (C6) may be expressed as the product of an $\mathrm{N}$ by 6 matrix with the vector of parameters $\lambda_{\mathrm{k}}$ :

$$
c_{i}=\left\{\mathrm{SP}_{\mathrm{ik}}\right\} \begin{array}{r}
\mathrm{N} \times 6 \\
\lambda_{\mathrm{k}}
\end{array}
$$

This is the basic relation for evaluating the coefficients for the six parameter spline.

For the nine parameter spline, a relation similar to equation (C4) is used but the spline is made to fit exactly the value of $\lambda_{k}$ which corresponds to the center value of the panel for which we are seeking the doublet distribution. A weighted least squares fit is then made with the values of $\lambda$ for the adjoining panels. It was found that smoother pressure distributions were obtained when the weights for the upstream panels were increased, with the highest weights (about 7) for the panels directly upstream. This weighting recognizes the predominance of upstream influences in supersonic flows.

As was shown in section 6, it is essential that the doublet strength be continuous across all panel edges if we are to avoid the infinite singularities emanating into the flow field from panel edges. This is accomplished by dividing the panel into eight subpanels and defining a quadratic distribution over each subpanel. The determination is accomplished in the stages following.

For each panel, nine doublet strength parameters are defined as the values of the doublet strength at the nine vertices of the eight triangular subpanels strength at the nine vertices of the eight triangular subpanels by using equations (C3) and (C7). Since the definitions of 8 of these doublet strength parameters are associated with points on the boundaries between adjacent panels, they are exactly the same for the adjoining panels. It will be shown that this achieves the desired continuity of doublet strength across panel boundaries for a quadratic doublet distribution.

Six of the ten straight lines which bound subpanels contain three subpanel vertices. In figure 96 these are the lines ABC, DEF, GHI, ADG, BEH, and CFI. The doublet strength parameters associated with the vertices may, therefore, be fitted with quadratics along each of these six lines. 
Since three doublet strength values along each line uniquely determine a quadratic, the doublet strength on a panel edge not only agrees with the doublet strength on the adjoining edge at the three common points, but also at all other points of the line. By using piecewise quadratic distributions in the eight subpanels of figure 95 , we can construct a nine parameter spline which is continuous everywhere in a network. This can be easily seen by considering figure 97 , which shows values of doublet strength interpolated by the quadratic at the midpoints of the subpanels edges. Every subpanel has six values of the doublet strength which uniquely defines the quadratic inside the triangle. As seen in figure 98 , continuity across subpanel edges is assured since the resulting quadratics from adjacent subpanels are uniquely defined on the panel edges by the three common values of doublet strength.

In place of actually determining the doublet strength at subpanel midpoints, a quantity is defined for each edge which provides the required additional information. This quantity is

$$
\begin{aligned}
\mathrm{K}_{\mathrm{ij}} & =\mu\left(\overline{\mathrm{P}}_{\mathrm{i}}\right)+\nabla \mu\left(\overline{\mathrm{P}}_{\mathrm{i}}\right) \cdot\left(\overline{\mathrm{P}}_{\mathrm{j}}-\overline{\mathrm{P}}_{\mathrm{i}}\right) / 2 \\
& =\mu\left(\overline{\mathrm{P}}_{\mathrm{j}}\right)+\nabla \mu\left(\overline{\mathrm{P}}_{\mathrm{j}}\right) \cdot\left(\overline{\mathrm{P}}_{\mathrm{i}}-\overline{\mathrm{P}}_{\mathrm{j}}\right) / 2
\end{aligned}
$$

where $P_{i}$ and $P_{j}$ are the position vectors of the two endpoints of the subpanel edge. For a quadratic distribution, these two forms can be shown to be equal. Using equation (C3) for $\mu$ with points $\zeta_{\mathrm{i}}, \eta_{\mathrm{i}}$ and $\zeta_{\mathrm{i}}$, we obtain

$$
\begin{aligned}
\mathrm{K}_{\mathrm{ij}}=\mu_{0} & +\mu_{\mathrm{x}}\left(\xi_{\mathrm{i}}+\xi_{\mathrm{j}}\right) / 2+\mu_{\mathrm{y}}\left(\eta_{\mathrm{i}}+\eta_{\mathrm{j}}\right) / 2 \\
& +\mu_{\mathrm{xx}}\left(\xi_{\mathrm{i}} \xi_{\mathrm{j}}\right) / 2+\mu_{\mathrm{xy}}\left(\xi_{\mathrm{i}} \eta_{\mathrm{j}}+\xi_{\mathrm{i}} \eta_{\mathrm{i}}\right) / 2 \\
& +\mu_{\mathrm{yy}} \eta_{\mathrm{i}} \eta_{\mathrm{j}} / 2
\end{aligned}
$$

which is seen to be symmetric in $\mathrm{i}$ and $\mathrm{j}$. These quantities are obtained on the 12 subpanel edges corresponding to the lines DEF, GHI, ADG, BEH, and CFI by using equations (C3) and with the coefficients defined by equation (C7). Since the gradient of a piecewise quadratic is not necessarily continuous everywhere, we approximate the $\mathrm{K}_{\mathrm{ij}}$ value for the edges $\mathrm{DH}, \mathrm{HF}$, $\mathrm{FB}$, and $\mathrm{BD}$ by a linear combination of the $\mathrm{K}_{\mathrm{ij}}$ for surrounding edges. For example, the value of $K_{i j}$ for the side $\mathrm{DH}$ is found by a linear combination of the $\mathrm{K}_{\mathrm{ij}}$ for the sides DG, $\mathrm{GH}$, $\mathrm{DE}$, and HE. For this evaluation we need to develop parameters associated with the departure of the panel from a parallelogram.

We now calculate parameters $C_{\mathfrak{i}} j$ which measure the skewness of the panel. Referring to figure 98 we define the vector $\overline{\mathrm{P}}_{\mathrm{mn}}=\overline{\mathrm{P}}_{\mathrm{m}}-\overline{\mathrm{P}}_{\mathrm{n}}$, where $\overline{\mathrm{P}}_{\mathrm{m}}$ and $\overline{\mathrm{P}}_{\mathrm{n}}$ are the position vectors of the corner points. Then, for the upper quadrilateral of the panel, ${ }_{1}{ }_{1} P_{6} P_{9} P_{5}$ in figure 98 , we define constants $C_{1}{ }^{1}$ such that

$$
\widetilde{\bar{P}}_{19}=\left(1+C_{1}{ }^{1}\right) \widetilde{\bar{P}}_{59}+\left(1+C_{2}{ }^{1}\right) \widetilde{\bar{P}}_{69}
$$


where the tilda denotes the projection of the point on the average plane. If $C_{1} 1=C_{2} 1=0$, then the quadrilateral is a parallelogram. We eliminate $\mathrm{C}_{2}$ by performing the cross product of the equation with $\mathrm{P}_{69}$ and obtain

$$
\widetilde{\overline{\mathrm{P}}}_{69} \times\left(\widetilde{\overline{\mathrm{P}}}_{19}-\widetilde{\overline{\mathrm{P}}}_{59}\right)=\mathrm{C}_{1} 1\left(\widetilde{\widetilde{\mathrm{P}}}_{69} \times \widetilde{\overline{\mathrm{P}}}_{59}\right)
$$

Now $\mathrm{P}_{19}=\mathrm{P}_{59}+\mathrm{P}_{15}$, and since the crossproducts are vectors normal to the plane (or effectively scalars), we obtain for $\mathrm{C}_{1} 1$

$$
C_{1} 1=\frac{\widetilde{\overline{\mathrm{P}}}_{69} \times \widetilde{\overline{\mathrm{P}}}_{15}}{\widetilde{\widetilde{\mathrm{P}}}_{69} \times \widetilde{\widetilde{\mathrm{P}}}_{59}}
$$

Now the tilda marked vectors differ from the complete vectors by components normal to the average plane, hence, the value of $C_{1}{ }^{1}$ can be expressed in terms of the points $\overline{\mathrm{P}}_{\mathbf{j}}$ by

$$
C_{1} 1=\frac{\left(\overline{\mathrm{P}}_{69} \times \overline{\mathrm{P}}_{15}\right) \cdot \hat{\mathrm{n}}}{\left(\overline{\mathrm{P}}_{69} \times \overline{\mathrm{P}}_{59}\right) \cdot \hat{\mathrm{n}}}
$$

since the contributions to the numerator and denominator from the normal components of the vectors vanish. Similarly, eliminating $C_{1}{ }^{1}$ by the cross product of the equation with $\overline{\mathrm{P}}_{59}$ yields

$$
\mathrm{C}_{2} 1=\left(\widetilde{\overline{\mathrm{P}}}_{16} \times \widetilde{\overline{\mathrm{P}}}_{59}\right) /\left(\widetilde{\overline{\mathrm{P}}}_{69} \times \widetilde{\overline{\mathrm{P}}}_{59}\right)
$$

since $\mathrm{P}_{19}=\mathrm{P}_{69}+\mathrm{P}_{16}$. For the remaining corners, we take the points $\mathrm{P}_{2} \mathrm{P}_{6} \mathrm{P}_{9} \mathrm{P}_{7}, \mathrm{P}_{3} \mathrm{P}_{8} \mathrm{P}_{9} \mathrm{P}_{7}$, and $\mathrm{P}_{4} \mathrm{P}_{8} \mathrm{P}_{9} \mathrm{P}_{5}$ and obtain the following formulas for $\mathrm{C}_{\mathrm{j}} \mathrm{j}$ :

$$
\begin{aligned}
& \mathrm{C}_{1} 1=\frac{\left(\overline{\mathrm{P}}_{69} \times \overline{\mathrm{P}}_{15}\right) \cdot \hat{n}}{\left(\overline{\mathrm{P}}_{69} \times \overline{\mathrm{P}}_{59}\right) \cdot \hat{n}} \\
& \mathrm{C}_{1}{ }^{2}=\frac{\left(\overline{\mathrm{P}}_{69} \times \overline{\mathrm{P}}_{27}\right) \cdot \hat{n}}{\left(\overline{\mathrm{P}}_{69} \times \overline{\mathrm{P}}_{79}\right) \cdot \hat{n}} \\
& \mathrm{C}_{1}{ }^{3}=\frac{\left(\overline{\mathrm{P}}_{89} \times \overline{\mathrm{P}}_{37}\right) \cdot \hat{n}}{\left(\overline{\mathrm{P}}_{89} \times \overline{\mathrm{P}}_{79}\right) \cdot \hat{n}} \\
& \mathrm{C}_{1}^{4}=\frac{\left(\overline{\mathrm{P}}_{89} \times \overline{\mathrm{P}}_{45}\right) \cdot \hat{n}}{\left(\overline{\mathrm{P}}_{89} \times \overline{\mathrm{P}}_{59}\right) \cdot \hat{n}}
\end{aligned}
$$


for $\mathrm{C}_{2} \mathrm{j}$,

$$
\begin{aligned}
& \mathrm{C}_{2} 1=\frac{\left(\overline{\mathrm{P}}_{16} \times \overline{\mathrm{P}}_{59}\right) \cdot \hat{\mathrm{n}}}{\left(\overline{\mathrm{P}}_{69} \times \overline{\mathrm{P}}_{59}\right) \cdot \hat{\mathrm{n}}} \\
& \mathrm{C}_{2}{ }^{2}=\frac{\left(\overline{\mathrm{P}}_{26} \times \overline{\mathrm{P}}_{79}\right) \cdot \hat{\mathrm{n}}}{\left(\overline{\mathrm{P}}_{69} \times \overline{\mathrm{P}}_{79}\right) \cdot \hat{n}} \\
& \mathrm{C}_{2}{ }^{3}=\frac{\left(\overline{\mathrm{P}}_{38} \times \overline{\mathrm{P}}_{79}\right) \cdot \hat{\mathrm{n}}}{\left(\overline{\mathrm{P}}_{89} \times \overline{\mathrm{P}}_{79}\right) \cdot \hat{\mathrm{n}}} \\
& \mathrm{C}_{2} 4=\frac{\left(\overline{\mathrm{P}}_{48} \times \overline{\mathrm{P}}_{59}\right) \cdot \hat{\mathrm{n}}}{\left(\overline{\mathrm{P}}_{89} \times \overline{\mathrm{P}}_{59}\right) \cdot \hat{\mathrm{n}}}
\end{aligned}
$$

We are now able to define the $\mathrm{K}_{\mathrm{ij}}$ quantities for the subpanel edges $\mathrm{DH}, \mathrm{HF}, \mathrm{FB}$, and $\mathrm{BD}$ in figure 96. Let $F_{i}=1+C_{1}{ }^{i}+C_{2}{ }^{i}$. For the value of $K_{58}$ and side $P_{5}-P_{8}$ in figure 98 , we have

$$
\mathrm{K}_{58}=\frac{\mathrm{F}_{1}}{1+\mathrm{F}_{1}}\left(\mathrm{~K}_{59}+\mathrm{K}_{89}\right)+\frac{1}{1+\mathrm{F}_{1}}\left(\mathrm{~K}_{51}+\mathrm{K}_{81}\right)
$$

Similar results may be written down for the other edges, $\mathrm{P}_{5}-\mathrm{P}_{6}, \mathrm{P}_{6}-\mathrm{P}_{7}$, and $\mathrm{P}_{7}-\mathrm{P}_{8}$.

For each triangular subpanel we now have the values of the doublet strength at the corner points and values of $\mathrm{K}_{\mathrm{ij}}$ for each of the sides. The coefficients of the quadratic in equation (C3) then can be expressed in terms of these six quantities by

$$
\left(\begin{array}{l}
\mu_{1} \\
\mu_{2} \\
\mu_{3} \\
\mathrm{~K}_{1} \\
\mathrm{~K}_{2} \\
\mathrm{~K}_{3}
\end{array}\right)=\mathrm{D} \quad\left(\begin{array}{l}
\mu_{\mathrm{o}} \\
\mu_{\mathrm{x}} \\
\mu_{\mathrm{y}} \\
\mu_{\mathrm{xx}} \\
\mu_{\mathrm{xy}} \\
\mu_{\mathrm{yy}}
\end{array}\right)
$$


or

$$
\left(\begin{array}{l}
\mu_{\mathrm{o}} \\
\mu_{\mathrm{x}} \\
\mu_{\mathrm{y}} \\
\mu_{\mathrm{xx}} \\
\mu_{\mathrm{xy}} \\
\mu_{\mathrm{yy}}
\end{array}\right)=\mathrm{D}^{-1}\left(\begin{array}{l}
\mu_{1} \\
\mu_{2} \\
\mu_{3} \\
\mathrm{~K}_{1} \\
\mathrm{~K}_{2} \\
\mathrm{~K}_{3}
\end{array}\right)
$$

where $\mu_{1}, \mu_{2}, \mu_{3}$ are the corner values of the doublet strength on the triangular subpanels, and $\mathrm{K}_{1}, \mathrm{~K}_{2}, \mathrm{~K}_{3}$ are the values of the parameter $\mathrm{K}_{\mathrm{ij}}$ for the three sides of the subpanel. The matrix $\mathrm{D}$ is given by

$$
\left(\begin{array}{lllll}
1 \xi_{1} & \eta_{1} & \xi_{1}^{2} / 2 & \xi_{1} \eta_{1} & \eta_{1}{ }^{2 / 2} \\
1 \xi_{2} & \eta_{2} & \xi_{2}{ }^{2 / 2} & \xi_{2} \eta_{2} & \eta_{2}^{2} / 2 \\
1 \xi_{3} & \eta_{3} & \xi_{3}^{2 / 2} & \xi_{3} \eta_{3} & \eta_{3}^{2} / 2 \\
1 \frac{\xi_{1}+\xi_{2}}{2} & \frac{\eta_{1}+\eta_{2}}{2} & \frac{\xi_{1} \xi_{2}}{2} & \frac{1}{2}\left(\begin{array}{c}
\xi_{1} \eta_{2} \\
+\xi_{2} \eta_{1}
\end{array}\right) \frac{\eta_{1} \eta_{2}}{2} \\
1 \frac{\xi_{2}+\xi_{3}}{2} & \frac{\eta_{2}+\eta_{3}}{2} & \frac{\xi_{2} \xi_{3}}{2} & \frac{1}{2}\left(\begin{array}{c}
\xi_{2} \eta_{3} \\
+\eta_{2} \xi_{3}
\end{array}\right) \frac{\eta_{2} n_{3}}{2} \\
1 \frac{\xi_{1}+\xi_{3}}{2} & \frac{\eta_{1}+\eta_{3}}{2} & \frac{\xi_{3} \xi_{1}}{2} & \frac{1}{2}\left(\begin{array}{c}
\xi_{1} \eta_{3} \\
+\xi_{3} \eta_{1}
\end{array}\right) \frac{\eta_{1} \eta_{3}}{2}
\end{array}\right)
$$

The matrix D may be inverted whenever the triangular subpanel does not degenerate into a line, for which we do not need the coefficients for $\mu$.

Hence, within each panel we have a continuous piecewise quadratic representation of the doublet strength in terms of the nine doublet strength parameters. The doublet strength is also continuous with the doublet strength on neighboring panels.

We shall now explain how the nine values of the doublet strength at the vertices of the eight panel subpanels in figure 96 are obtained. As in the case of the source distribution, it is convenient to regard as the basic unknowns the values of the doublet strength at the panel centers. This helps to assure a correspondence between the number of unknowns and the number of boundary conditions. For panels well within the network interior, the doublet strength parameter at a corner of a panel is determined by least-squares fitting of the quadratic in equation (C3) to the doublet strength at the centers of 12 nearby panels, as 
shown in figure 99a by a means of equation (C7). The squared errors at the four panel mispoints closest to the corner point in question are given a weight of $10^{3}$ while the outer . uncircled points are weighted according to a formula which gives greater weight to points upstream of the point where the doublet strength is desired, namely

$$
\mathrm{w}_{\mathrm{k}}=1+\mathrm{M}\left(1-\mathrm{R}_{\mathrm{k}} \cdot \hat{\mathrm{C}}\right)
$$

where $R_{k}$ is the vector distance between the central point and the kth outer point and $\hat{C}$ is the unit vector in the compressible direction (usually the freestream direction). Similarly, the doublet strength parameters at the midpoint of a panel edge is found by least square fitting a quadratic to the doublet strength at the centers of 12 nearby panels, the squared errors at the centers of the two panels bordering the edge being weighted at $10^{3}$ while the other points are weighted according to the formula. These arrangements are illustrated in figure $99 \mathrm{a}$ and $99 \mathrm{~b}$.

The doublet strengths at panel corner points along network edges are required for the nine parameter spline. Because of the necessity of matching doublet strength across network edges, the determination of the doublet strength at panel corners on a network edge must be confined to the valucs of the doublet parameters along the cdge. We recall from figure 14 that singularities are provided at the midpoints of the panel edges bordering networks and at network corners. The corner values are the actual doublet strength. The midpoint edge values are the doublet strength for the six parameter spline; but for the nine parameter spline we use, instead of the doublet strength, the quantity

$$
\begin{aligned}
\lambda_{\mathrm{i}} & =\mu\left(\overline{\mathrm{P}}_{\mathrm{i}-1}\right)+\frac{1}{2} \nabla \mu\left(\overline{\mathrm{P}}_{\mathrm{i}-1}\right) \cdot\left(\overline{\mathrm{P}}_{\mathrm{i}}-\overline{\mathrm{P}}_{\mathrm{i}-1}\right) \\
& =\mu\left(\overline{\mathrm{P}}_{\mathrm{i}}\right)+\frac{1}{2} \nabla \mu\left(\overline{\mathrm{P}}_{\mathrm{i}}\right) \cdot\left(\overline{\mathrm{P}}_{\mathrm{i}-1}-\overline{\mathrm{P}}_{\mathrm{i}}\right)
\end{aligned}
$$

In terms of these parameters, the corner values of doublet strength are given by

$$
\mu\left(\overline{\mathrm{P}}_{i}\right)=\frac{\left|\overline{\mathrm{P}}_{\mathrm{i}}-\overline{\mathrm{M}}_{\mathrm{i}-1}\right| \lambda_{\mathrm{i}+\mathrm{i}}}{\left|\overline{\mathrm{P}}_{\mathrm{i}}-\overline{\mathrm{M}}_{\mathrm{i}-1}\right|+\left|\overline{\mathrm{M}}_{\mathrm{i}}-\overline{\mathrm{P}}_{\mathrm{i}}\right|}+\frac{\left|\overline{\mathrm{M}}_{\mathrm{i}}-\overline{\mathrm{P}}_{\mathrm{i}}\right| \lambda_{\mathrm{i}}}{\left|\overline{\mathrm{P}}_{\mathrm{i}}-\overline{\mathrm{M}}_{\mathrm{i}-1}\right|+\left|\overline{\mathrm{M}}_{\mathrm{i}}-\overline{\mathrm{P}}_{\mathrm{i}}\right|}
$$

where $P_{i}$ denotes the cornerpoint and $\bar{M}_{\mathfrak{i}}$ the midpoints as shown in figure 100 . This can be shown easily by substituting $\lambda_{i+1}$ by the first of equations (C14) and $\lambda_{i}$ by the second, while noting that the gradient terms cancel. 
Equation (C15) is equivalent to straightening the edge and using a single variable to represent $\mu$ along the edge. In terms of a single variable, then equation (C14) takes the form

$$
\lambda_{i+1}=\mu_{i+1}+\frac{1}{2}\left(\frac{d \mu}{d x}\right)_{i+1}\left(x_{i}-x_{i+1}\right)
$$

Since we require $\mu$ at the midpoint, we let $x_{i+1}$ and $x_{i}$ be \pm 1 , respectively. Then along the panel edge, the doublet strength varies as

$$
\mu(\mathrm{x})=\mu_{\mathrm{m}}+\frac{\mu_{\mathrm{i}+1}-\mu_{\mathrm{i}}}{2} \mathrm{x}+\left(\frac{\mu_{\mathrm{i}+1}+\mu_{\mathrm{i}}}{2}-\mu_{\mathrm{m}}\right) \mathrm{x}^{2}
$$

where $\mu_{\mathrm{m}}$ denotes the value of the doublet strength at the midpoint of the panel edge. From equation $(\mathrm{C} 17)$, we have

$$
\left(\frac{\mathrm{d} \mu}{\mathrm{dx}}\right)_{\mathrm{i}+1}=\frac{3 \mathrm{u}_{\mathrm{i}+1}+\mu_{\mathrm{i}-1}}{2}-2 \mu_{\mathrm{m}}
$$

which gives for equation (C16), after solving for $\mu_{\mathrm{m}}$,

$$
\mu_{\mathrm{m}}=\mu_{\mathrm{i}+1} / 4+\mu_{\mathrm{i}} / 4+\lambda_{\mathrm{i}+1} / 2
$$

Thus, the panel comer values and panel midpoint values of the doublet strength are determined by the singularity parameters $\lambda_{i}$ at the panel midpoints on the network edge by equations (C15) and (C18).

With the values of the doublet strength at panel corners of the network edge defined, we are now able to compute the corner and midpoint values for all the panels in the network for application to the nine parameter spline. For the panels near the network edges, we obtain the corner values of the doublet strength by using equation (C3) and (C7) to obtain a least squares fit with neighboring panel center values and with network edge values.

Figure 99 shows the fit for interior panels well within the network. For panels close to the network edge, the panel corner and edge midpoint values are computed with a least square fit with neighboring doublet strength values which also include corner points lying on the network edge. Figures $101 \mathrm{a}$ and $101 \mathrm{~d}$ show a fit made with only ten points. However, the doublet strengths at the two corner panel points lying on the network edge are expressed in terms of the two basic parameters on the network edge at the panel midpoints of the adjoining panels. Thus, the vector of coefficients for equation (C3) is computed in terms of a matrix product of a 12 by 6 matrix and the neighboring $\lambda_{k}$ basic singularity parameters. In figures $101 \mathrm{~b}$ and $101 \mathrm{c}$ a least squares fit of eleven points is used. All cases result in a 12 by 6 matrix with the 12 basic singularity parameters. 
Naturally, special attention must be given in cases for which the panels are triangular rather than quadrilateral, if only because it is impossible to fit a quadratic to three values at a common point. However, the same basic selection of unknowns serves to define enough doublet strength parameters to fix piecewise continuous quadratics in each of the six subpanels of any triangular panel (see fig. 102). 


\section{APPENDIX D: \\ DESCRIPTION OF THE PILOT CODE FOR COMPUTING LINEARIZED SUPERSONIC FLOW OVER AIRCRAFT CONFIGURATIONS}

The basic formulas for the computation of supersonic flow by the improved higher order panel method have been derived in the foregoing sections. These derivations are detailed and somewhat lengthy with the formulas actually to be computed scattered throughout the document. To present an overall view of the method we shall describe, in some detail, the basic calculations used in the pilot code to compute linearized supersonic flow and refer to the equations and the sections in the text which are applicable. This section is not meant to be a description of inputs and procedures to be applied by the user in running the pilot code, since a document written for this purpose is available in reference [32].

Section 3 presents the basic theory of linearized supcrsonic flow following to some degree the work of G.N. Ward [10]. Section 4 gives a brief general description of the panel method. The aerodynamic influence coefficients are derived and a discussion of their properties is given in sections 5 through 9 for both the subinclined and superinclined panels. Some of the pure mathematical details have been relegated to the appendices A and B. Since the formulas for the influence coefficients were derived for subinclined panels parallel to the freestream direction and superinclined panels normal to the freestream, the influence coefficients must be transformed for panels at other inclinations by means of the transformation matrices derived in section 10. If panels are located well within the upstream Mach cone from a control point, then the computations can be madc morc cconomical by using the far field expansions described in section 11 .

\section{D1. BASIC GEOMETRY AND PANEL DEFINING QUANTITIES}

The first step in using a panel method is to divide the configuration into networks. The network boundaries are distinguished by discontinuities in the configuration geometry such as wing and body junctions. The spline representation of source and doublet assumes smoothly varying geometry so the body is divided into networks and appropriate matching conditions are used across network boundaries for the doublet strength. Networks also may be distinguished by the boundary conditions applied to them. For this reason, no network can contain both subinclined and superinclined panels.

Each network is divided into panels by a double array of grid points on the surface. The rows and columns are chosen so that the cross product of a vector in the column direction with the vector in the row direction is pointed in the direction of the positive outward normal to the surface into the fluid.

The boundary conditions are applied at the center of the panel and for this we requirc the unit normal vector. The panel normal is chosen as the normal to the average plane or to the central parallelogram of figure 98 . This is defined as the cross product of the vectors formed by the lines joining the midpoints of the sides of the parallelogram or 


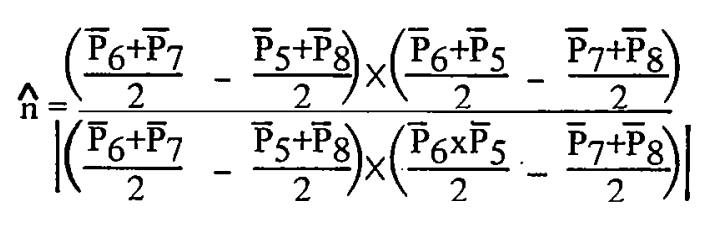

Normals are also defined for the subpanels. Although these are not used for boundary conditions, they are used to define the transformation matrices required for the aerodynamic influence coefficients of each subpanel. Also computed is the center of each panel or subpanel. It is defined as the average of the corner points and is the origin of the coordinates in which the formulas for the aerodynamic influence coefficients are expressed. For supersonic flow, one of these coordinates is aligned with the projection of the freestream velocity on the panel.

The control points at which boundary conditions are applied are then computed, the locations of which are illustrated in figures 13 and 14 for the source and doublet networks with analysis boundary conditions. For the nine parameter spline, the panel center control point must be away from exact center because of this point being a common vertex to the four central subpanels of figure 98 .

The control points at the edges of doublet networks are used for matching doublet strength across network boundaries. For the 6 parameter spline and for earlier versions of the nine-parameter spline, the edge matching control points are moved into the network an infinitesimal distance. This is essential since the infinite singularity at the panel edge in section 6 is used in the six parameter spline to match the doublet strength at network boundaries. For the nine parameter spline, the continuity of the doublet strength allows us to drop all line vortices at panel edges and in the earlier version, a simulated incompressible vortex is introduced at network edges as described in section 4 with equations (4.1) and (4.2) to match doublet strength. The latest version matches doublet strength exactly by prescribing regular downwash boundary conditions at the edge control points for the upstream network to determine the doublet strength and using these values in the downstream network, to obtain exact matching of the doublet strength across the two networks.

Up to this point, the calculations are carried out without regard to whether the flow is subsonic or supersonic or for any choice of Mach number. In summary, we have computed the following quantities:

1. Designation of networks and the double array of panel corner points on the surface corresponding to each network.

2. Additional points of each panel used to define the eight triangular subpanels.

3. Unit normals to each panel and subpanel.

4. Location of the control points at panel centers for the boundary conditions and control points at network edges for matching doublet strength. 


\section{D2. TRANSFORMATION MATRICES}

The aerodynamic influence coefficients were developed for a scaled coordinate system with subinclined panels parallel to the free stream and superinclined panels normal. To calculate the transformations which convert the aerodynamic influence coefficients for any general inclined panel, we must introduce the Mach number at which the pressures and velocities are desired.

These transformations were derived in section 10 . With $x_{p}, y_{p}, z_{p}$ designating the coordinate system on the subpanel whose origin is at the subpanel center, the transformation from a reference system $x_{r}, y_{r}, z_{r}$ to the panel system is described by the equations (10.37) (10.38), and (10.39) or

$$
\left(x_{p}, y_{p}, z_{p}\right)=\left\{A_{2}\right\}\left\{A_{1}\right\}\left\{A_{r}\right\} \quad\left(\begin{array}{l}
x_{r}-x_{o} \\
y_{r}-y_{o} \\
z_{r}-z_{o}
\end{array}\right) .
$$

where $\mathrm{x}_{0}, \mathrm{y}_{0}, \mathrm{z}_{0}$ are the coordinates of the subpanel center in reference coordinates.

For source influence coefficients there is a correction required which is given by the Jacobian of the transformation from the reference system to the panel coordinate system in equations (10.42) for subinclined panels and (10.44) for superinclined panels.

\section{D3. CALCULATION OF AERODYNAMIC INFLUENCE COEFFICIENTS}

The influence coefficients to be calculated depend upon the boundary conditions that are to be applied to the surface. The pilot code is set up on the assumption of a combined source and doublet panel system. A single singularity surface is selected by setting the unrequired singularity equal to zero. The most likely boundary conditions are the potential type boundary conditions, in which the source strength $\sigma$ is defined to cancel the the normal component of the free stream velocity on the outer surface, i.e.,

$$
\sigma=-\overline{\mathrm{U}} \cdot \hat{\mathrm{n}}
$$

and the perturbation potential on the interior surface of the configuration is set equal to zero, or

$$
\phi^{-}=0
$$

These boundary conditions were shown in section 3.8 to satisfy the vanishing of the normal component of the linear mass flux on the outer surface and to eliminate the interior flow perturbations. Equation (D3) leads to a set of simultaneous linear equations to be solved for the doublet parameters. 
Since the source parameters are defined by equation (D2) they contribute to the right hand side of the simultaneous equations.

The boundary conditions in equation (D3) is applied to the downstream network edge. On the upstream network edge of the adjoining network we apply the boundary condition in equation (4.1).

The contribution from a panel edge to the potential from a quadratic distribution of the doublet strength is given in equation (5.17) where the functions are derived in Appendix A. The contribution of the entire subpanel to the perturbation potential at the control point is found by evaluating and summing equation (5.17) for each end point of the side and for. each side by proceding counter-clockwise around the panel. The quantity $\mu$ and its derivatives are defined in equation (C3) evaluated for the $\mathrm{x}, \mathrm{y}, \mathrm{z}$ coordinates of the control point where the boundary conditions are to be computed. Substituting equation (C3) for $\mu$ into equation (5.17) and combining coefficients yields a relation of the form

$$
\phi=\mathrm{A}_{\mathbf{i}} \mathbf{c}_{\mathbf{i}}
$$

where the vector $c_{i}$ is defined in equalion (C5). The contribution to the velocity potential from the panel can be expressed in terms of the basic parameters at the panel centers and network edges by equation (C7) yielding

$$
\phi=\mathrm{A}_{\mathrm{i}}\left\{\mathrm{SP}_{\mathrm{ik}}\right\} \mathrm{N} \times 6 \lambda_{\mathrm{k}}
$$

A similar relation holds for the contribution to $\phi$ from the source distribution; but with the $\lambda_{k}$ for the source panels known from the boundary conditions of equation (D2), it contributes to the right hand side of the boundary condition equation (D3). The potential for a panel edge from the source distribution is given in equation (8.6). The contribution to the entire subpanel or panel is found by evaluating and summing equation (8.6) for each edge and at both endpoints proceding around the panel in a counter-clockwise direction in the same way as for the doublet panel.

The pilot code computes the coefficients of the $\lambda_{k}$ in equation (D5) for each panel at all the control points before proceding to the next panel. A panel is selected and the control points for all the panels are transformed in to the coordinate system of the subpanel by equation (D1). Then a test is made to determine if the selected panel lies within or is cut by the Mach cone emanating upstream from the control point. The aerodynamic influence coefficients are not computed for those panels lying outside of the mach cone. The contribution from the selected panel is computed and stored for each control point. Then the program takes the next panel in order, repeats the procedures, and sums the results with the previous calculations for each control point. 
Similar procedures are followed when velocity components are computed at the control points. The velocity components for the quadratic doublet distribution are given in equation (6.1) for the six parameter spline and in equation (7.4), (7.6), and (7.9) for the nine parameter spline, in which the line vortices have been removed. The result of the computations of the boundary conditions in equation (D2) and (D3) lead to a set of simultaneous equations of the form

$$
\sum_{\mathrm{k}=1}^{N_{\mathrm{d}}} A_{\mathrm{ik}} \lambda_{\mathrm{k}}=\sum_{\mathrm{k}=1}^{\mathrm{N}_{\mathrm{S}}} B_{\mathrm{ik}}\left(\overline{\mathrm{U}} \cdot \hat{\mathrm{n}}_{\mathrm{k}}\right) \quad \mathrm{i}=1,2, \ldots \mathrm{N}_{\mathrm{d}}
$$

to be solved for the doublet parameters $\lambda_{k}$. Here, $N_{d}$ and $N_{s}$ are the numbers of doublet parameter and source parameters, respectively. Once the $\lambda_{k}$ are known, a similar procedure is used to compute the velocity components and the pressure at each control point.

For the combined source and doublet panels with the boundary conditions of equations (D2) and (D3), the tangential perturbation velocity components on the outer surface can be computed by differentiating the doublet strength on the panel. With the transformation from reference coordinate to panel coordinate system given by equation (D1), the tengential velocity vector $V_{\mathrm{r}}$ in the reference system is given in terms of the subpanel tangential velocity $\mathrm{V}_{\mathrm{p}}$ by

$$
\overline{\mathrm{V}}_{\mathrm{r}}=\left\{\mathrm{A}_{\mathrm{r}}\right\}^{\mathrm{T}}\left\{\mathrm{A}_{1}\right\}^{\mathrm{T}}\left\{\mathrm{A}_{2}\right\}^{\mathrm{T}} \overline{\mathrm{V}}_{\mathrm{p}}
$$

where the $T$ denotes the transpose of the matrix. The perturbation velocity is used to compute the pressure coefficient using equation (3.10) or the isentropic relation.

When the configuration also contains superinclined networks representing inlets, the procedure as outlined above is followed except the boundary conditions on the interior or downstream side of the network are

$$
\phi=\overline{\mathrm{W}} \cdot \hat{\mathrm{n}}=0
$$

Since there arc gencrally subinclined panels in the zone of dependence of the superinclined network, these boundary conditions yield relations similar to equation (D6) with the left hand side containing unknown source parameters as well as doublet.

The velocity potential for superinclined doublet panels is given in equations (9.12) and (9.16) with the functions $\mathrm{Q}_{\mathrm{I}}$ and $\mathrm{w}_{0}$ derived in Appendix $\mathrm{A}$, section $\mathrm{A}$ 9. The velocity potential for source panels is given in the equation following equation (9.16). The perturbation velocity components for the superinclined doublet panels are given in equations (9.30) and (9.31) and are used only in the nine parameter spline, since the line integrals of the doublet strength have been dropped. The velocity components from the superinclined source panel are presented at the end of section 9.3. 


\section{APPENDIX E: \\ RELATIONSHIP OF SUPERSONIC PANEL INFLUENCE COEFFICIENTS TO SUBSONIC PANEL INFLUENCE COEFFICIENTS}

The subsonic panel influence coefficients (ref. [3]) were obtained by evaluating the integrals of the form

$$
\mathrm{H}(\mathrm{M}, \mathrm{N}, \mathrm{K})=\iint_{\Sigma} \frac{(\xi-\mathrm{x})^{\mathrm{M}-1}(\eta-\mathrm{y})^{\mathrm{N}-1} \mathrm{~d} \xi \mathrm{d} \eta}{\mathrm{R}^{\mathrm{k}}}
$$

and

$$
F(M, N, K)=\int_{L} \frac{(\xi-x)^{M-1}(\eta-y)^{N-1} d \ell}{R^{k}}
$$

where

$$
R=\sqrt{(\xi-x)^{2}+(\eta-y)^{2}+h^{2}}
$$

Here $\Sigma$ is the panel surface (assumed to be flat for present purposes) and $\mathrm{L}$ is a typical edge. Also $(\mathrm{x}, \mathrm{y}, \mathrm{h})$ are the coordinates of the field point in the local panel coordinate system and $(\zeta, \eta, 0)$ are the corresponding coordinates of the integration point.

$e_{t}$

The in tegrals in equations (E1) and (E2) can be formally defined for a subinclined panel in supersonic tlow for which $\mathrm{R}$ becomes the hyperbolic distance defined by

$$
\mathrm{R}=\sqrt{(\xi-\mathrm{x})^{2}-(\eta-\mathrm{y})^{2}-\mathrm{h}^{2}}
$$

The $\mathrm{H}$ integrals satisfy three recursion relations obtained in the same manner as for subsonic flow. We have

$$
\begin{gathered}
H(M, N, K-2)=H(M+2, N, K)-H(M, N+2, K)-h^{2} H(M, N, K-2) \\
(K-2) H(M, N, K)=(M-2) H(M-2, N, K-2)-\sum_{1}^{4} \nu_{\xi} F(M, N-1, K-2)
\end{gathered}
$$




$$
(K-2) H(M, N, K)=-(N-2) H(M, N-2, K-2)+\sum_{1}^{4} \nu_{\xi} F(M, N-1, K-2)
$$

where the summation is over the panel edges. These recursions can be combined to produce the following, more useful recursions:

$$
\begin{aligned}
(M-N-K) H(M, N, K)= & h^{2}(M-2) H(M-2, N, K)-h^{2} \sum_{1}^{4} \nu_{\xi} F(M-1, N, K) \\
& +\sum_{1}^{4} \text { a F }(M, N, K)
\end{aligned}
$$

$$
\begin{aligned}
& (\mathrm{M}-\mathrm{N}-\mathrm{K}) \mathrm{H}(\mathrm{M}, \mathrm{N}, \mathrm{K})=-\mathrm{h}^{2}(\mathrm{~N}-2) \mathrm{H}(\mathrm{M}, \mathrm{N}-2, \mathrm{~K})+\mathrm{h}^{2}{\stackrel{\sum}{1} \nu_{\eta} \mathrm{F}(\mathrm{M}, \mathrm{N}-1, \mathrm{~K})}+\sum_{1}^{4} \text { a } \mathrm{F}(\mathrm{M}, \mathrm{N}, \mathrm{K}) \\
& (\mathrm{K}-2) \mathrm{h}^{2} \mathrm{H}(\mathrm{M}, \mathrm{N}, \mathrm{K})=(\mathrm{M}+\mathrm{N}-\mathrm{K}-2) \mathrm{H}(\mathrm{M}, \mathrm{N}, \mathrm{K}-2)-\sum_{1}^{4} \text { a } \mathrm{F}(\mathrm{M}, \mathrm{N}, \mathrm{K}-2)
\end{aligned}
$$

Here, a $\nu_{\xi}$ and $\nu_{\eta}$ are defined as in the subsonic case, i.e., $\hat{\nu}=\left(\nu_{\xi} \nu \eta\right)$ is the exterior normal to the edge $\ell$ and $a=\hat{\nu} \cdot(\xi-x, \eta-y)$.

The $\mathrm{F}$ integrals also satisfy three recursion relations obtained in the same manner as in subsonic flow. We have

$$
\begin{gathered}
F(M+2, N, K)-F(M, N+2, K)-h^{2} F(M, N, K)=F(M, N, K-2) \\
\nu_{\xi} F(M+1, N, K)+\nu_{\eta} F(M, N+1, K)=\text { a } F(M, N, K)
\end{gathered}
$$




$$
\begin{aligned}
& -(\mathrm{M}-1) \mathrm{F}(\mathrm{M}-1, \mathrm{~N}, \mathrm{~K}-2) \nu_{\eta}+(\mathrm{N}-1) \mathrm{F}(\mathrm{M}, \mathrm{N}-1, \mathrm{~K}-2) \nu_{\xi} \\
& +(\mathrm{K}-2) \mathrm{F}(\mathrm{M}+1, \mathrm{~N}, \mathrm{~K}) \nu_{\eta}+(\mathrm{K}-2) \mathrm{F}(\mathrm{M}, \mathrm{N}+1, \mathrm{~K}) \nu_{\xi}=\mathrm{E}(\mathrm{M}, \mathrm{N}, \mathrm{K}-2)
\end{aligned}
$$

where

$$
E(M, N, K)=\left.\frac{(\xi-x)^{M-1}(\eta-y)^{N-1}}{R^{k}}\right|_{1} ^{2}
$$

where the numbers 1 and 2 denote the endpoints of the subpanel edge. Because of the limited number of terms required for the flat panel supersonic case without line vortex terms no attempt has been made to recombine (E10), (E11), and (E12) into more useful recursion relations.

In order to evaluate the fundamental integrals $H(1,1,3)$ and $F(1,1,1)$ it is useful to make the following definitions. Let

$$
\mathrm{b}=\nu \xi^{2}-\nu_{\eta}{ }^{2}, \mathrm{~g}^{2}=\mathrm{a}^{2}-\mathrm{bh}^{2}, \text { and } \ell=\nu_{\eta}(\xi-\mathrm{x})+\nu \xi(\eta-\mathrm{y})
$$

Then

$$
\mathrm{R}^{2}=\left(\mathrm{g}^{2}-\ell^{2}\right) / \mathrm{b}
$$

and

$$
F(1,1,1)=\frac{1}{b} \int_{\ell_{1}}^{\ell_{2}} \frac{\mathrm{d} \ell}{\mathrm{R}}
$$

and

$$
H(1,1,3)=-\int_{\ell_{1}}^{\ell_{2}} \frac{\mathrm{ad} \ell}{\mathrm{R}\left(\mathrm{a}^{2}-\ell^{2}\right)}
$$

For linear source and quadratic doublet (without line vortex terms) influence coefficients $F(1,2,1)$ and $F(2,1,1)$ are also required, and these may be obtained via equations (E11) and (E12). Upon considerable analysis we obtain the following evaluations: 


$$
h H(1,1,3)=\left\{\begin{array}{l}
0, \quad h=0 \text { (point not on panel) } \\
\pi \operatorname{sign}(h \nu \xi), \quad R_{1}=R_{2}=0 \text { (wedge region) } \\
\tan ^{-1}\left[\mathrm{haF}_{1} /\left(\mathrm{R}_{1} \mathrm{R}_{2}+\mathrm{h}^{2} \mathrm{~F}_{2}\right)\right] \quad \text {, otherwise }
\end{array}\right.
$$

where

$$
\begin{aligned}
& F_{1}=\left\{\begin{array}{l}
\left(\ell_{1} R_{2}-\ell_{2} R_{1}\right) / g^{2}, b \geq 0 \\
\left(R_{2}^{2}-R_{1}^{2}\right) /\left(\ell_{1} R_{2}+\ell_{2} R_{1}\right), b \leq 0
\end{array}\right. \\
& F_{2}= \begin{cases}\left(b R_{1} R_{2}+\ell_{1} \ell_{2}\right) / g^{2} . & b \geq 0 \\
\left(g^{2}-\ell_{1} 2-\ell_{2} 2\right) /\left(b R_{1} R_{2}-\ell_{1} \ell_{2}\right) & b \leq 0\end{cases}
\end{aligned}
$$

and the subscripts 1 and 2 on $R$ and $\ell$ designate the quantities evaluated at the two ends of the side. Note that

$$
\mathrm{F}_{2}{ }^{2}+\mathrm{bF}_{1}{ }^{2}=1
$$

We also have

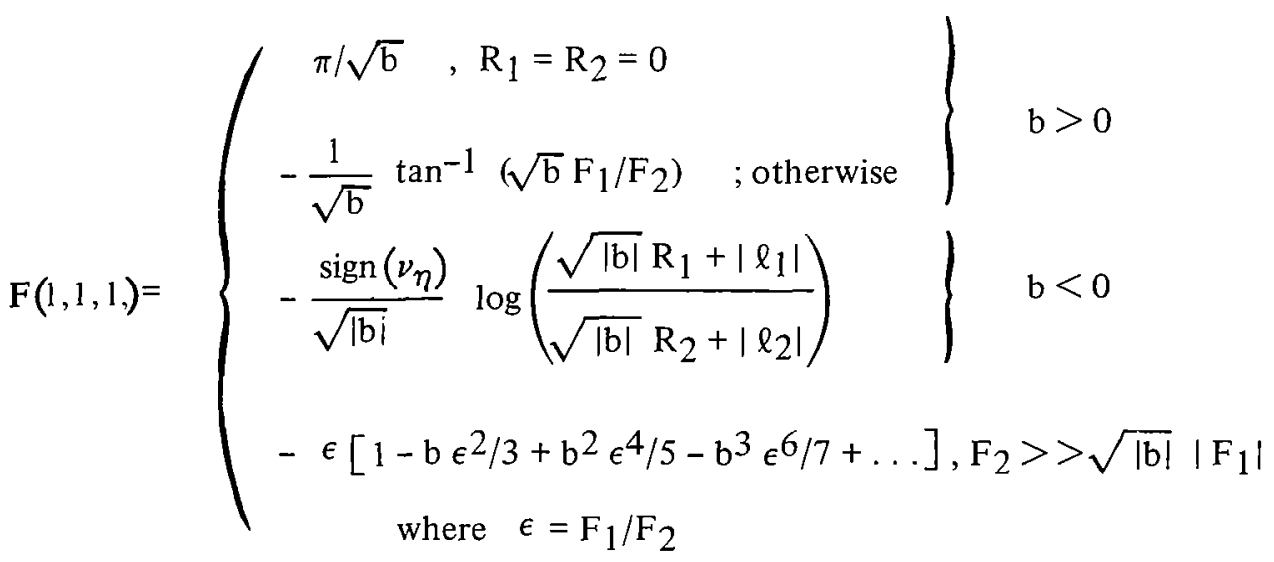


and

$$
\mathrm{F}(1,2,1)= \begin{cases}-\left[\nu_{\zeta}\left(\mathrm{R}_{2}-\mathrm{R}_{1}\right)+\mathrm{a} \nu_{\eta} \mathrm{F}(1,1,1)\right] / \mathrm{b} ; & \mathrm{b} \neq 0 \\ {\left[-\nu_{\zeta}\left(\mathrm{R}_{2}-\mathrm{R}_{1}\right) \mathrm{R}_{1} \mathrm{R}_{2}+\ell_{2} \mathrm{R}_{1}\left(\eta_{1}-\mathrm{y}\right)-\ell_{1} \mathrm{R}_{2}\left(\eta_{2}-\mathrm{y}\right)\right] / \mathrm{g}^{2} \mathrm{~F}_{2}} \\ -\mathrm{a} \nu_{\mathrm{n}} \epsilon^{3}\left[1 / 3-\mathrm{b} \epsilon^{2} / 5+\mathrm{b}^{2} \epsilon^{4} / 7+\ldots\right] & ; \mathrm{F}_{2}>>\sqrt{|\mathrm{b}|}\left|\mathrm{F}_{1}\right|\end{cases}
$$

and

$$
\mathrm{F}(2,1,1)=-\nu_{\eta}\left(\mathrm{R}_{2}-\mathrm{R}_{1}\right)+\mathrm{a} \nu_{\xi} \mathrm{F}(1,1,1)-2 \nu_{\xi} \nu_{\eta} \mathrm{F}(1,2,1)
$$

The relationship between the calculations of equations (1) through (24) and those of Appendix A will now be established through the following identities:

$$
\begin{aligned}
& \ell=\nu_{\eta}(\xi-\mathrm{x})+\nu_{\xi}(\eta-\mathrm{y})=-\nu_{\eta} \mathrm{s}_{\mathrm{m}}-\nu_{\xi} \mathrm{s}=\nu_{\xi}\left(\mathrm{s}_{\mathrm{m}} / \mathrm{m}-\mathrm{s}\right) \\
& \mathrm{d} \ell=\nu_{\xi}\left(1 / \mathrm{m}^{2}-1\right) \mathrm{ds} \\
& \lambda \quad=-\nu_{\eta} / \nu_{\xi} \\
& \mathrm{m} \quad=-\nu_{\xi} / \nu_{\eta} \\
& \mathrm{x}_{\mathrm{m}}=-\mathrm{a} / \nu_{\xi} \\
& \quad=\left(\mathrm{a} \nu_{\eta}-\ell \nu_{\xi}\right) / \mathrm{b} \\
& \mathrm{t}=\left(-\mathrm{a} \nu_{\xi}+\ell \nu_{\eta}\right) / \mathrm{b} \\
& \mathrm{Q}_{\mathrm{I}}=-\mathrm{hH}(1,1,3) \\
& \mathrm{w}_{\mathrm{O}}=-\nu_{\xi} \mathrm{F}(1,1,1) \\
& \lambda \mathrm{w}_{1}+\mathrm{x}_{\mathrm{m}} \mathrm{w}_{\mathrm{O}}=\nu_{\xi} \mathrm{F}(2,1,1) \\
& \mathrm{w}_{1}=\nu_{\xi} \mathrm{F}(1,2,1) \\
& \hat{\mathrm{x}}_{\mathrm{m}}=-\mathrm{a} / \nu_{\eta} \\
& \hat{\mathrm{w}}_{\mathrm{O}}=\nu_{\eta} \mathrm{F}(1,1,1) \\
& \hat{\mathrm{w}}_{1}=-\nu_{\eta} \mathrm{F}(1,2,1)
\end{aligned}
$$




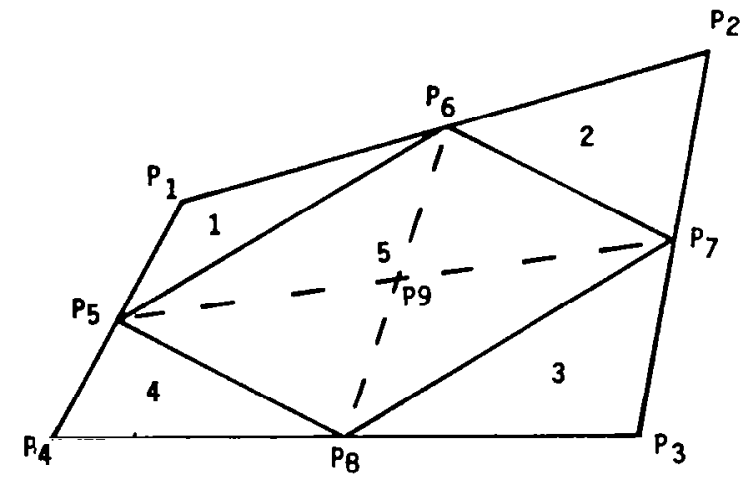

Figure 1.-Subpanel Structure of Basic Four Point Panel Showing The Five Planar Areas and Eight Triangular Subpanels

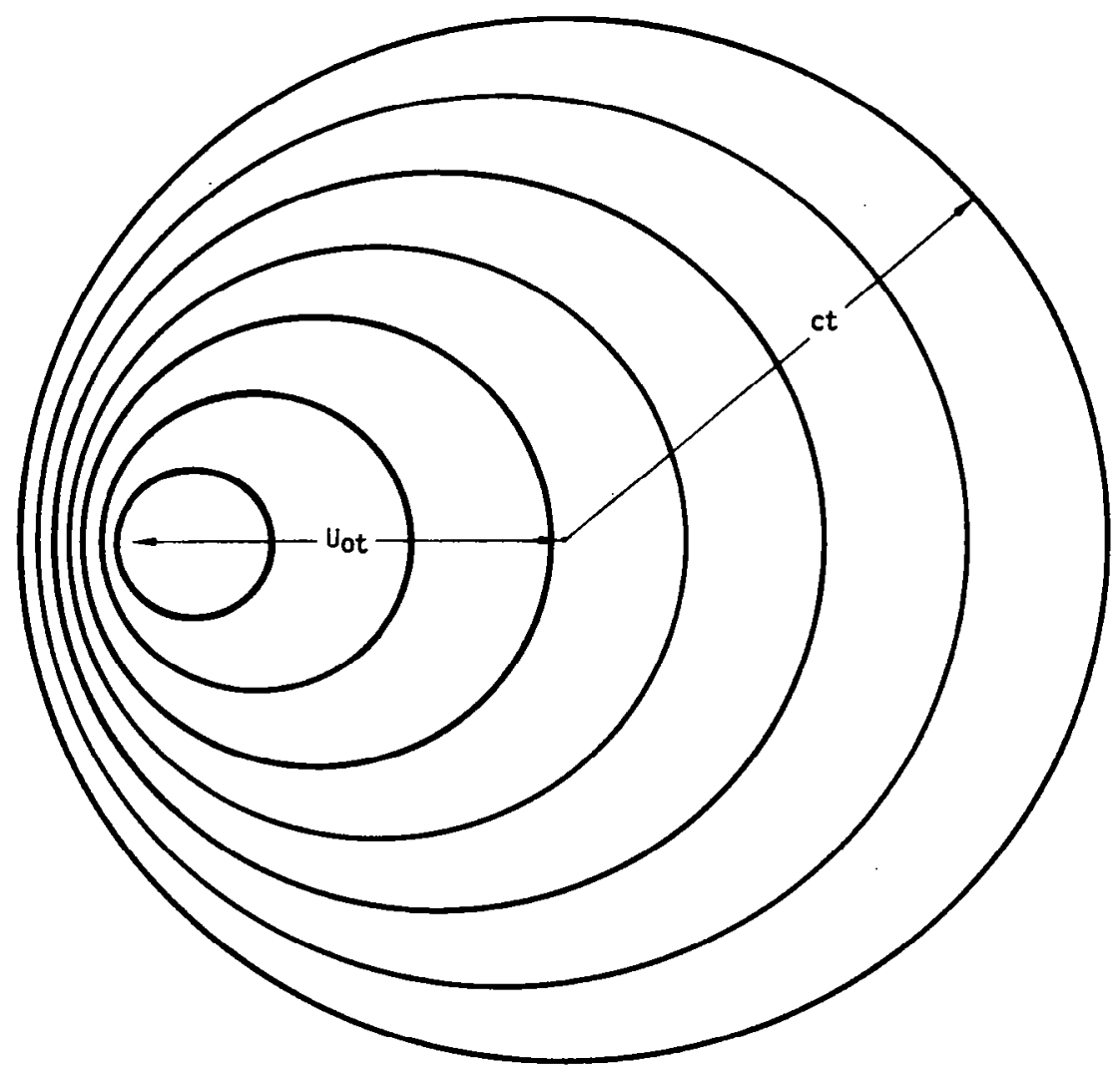

Figure 2.-Propagation of a Disturbance in Subsonic Flow 


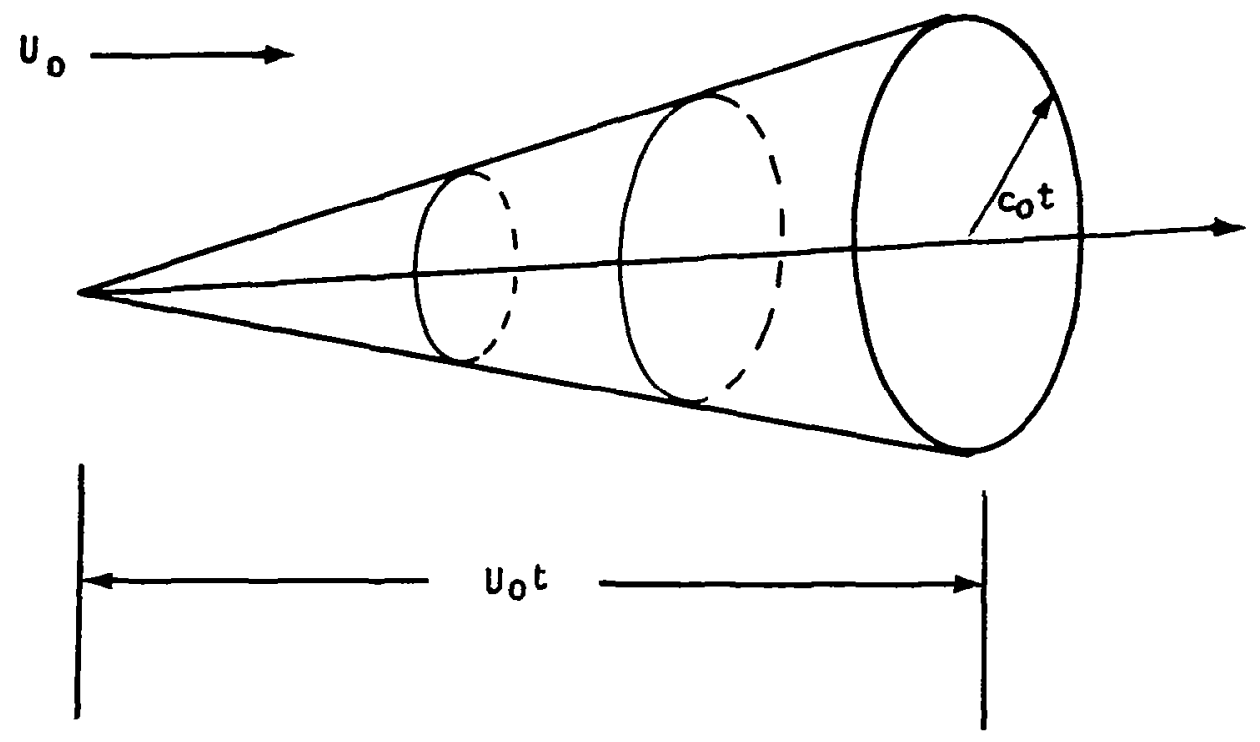

Figure 3.-Propagation of a Disturbance in Supersonic Flow

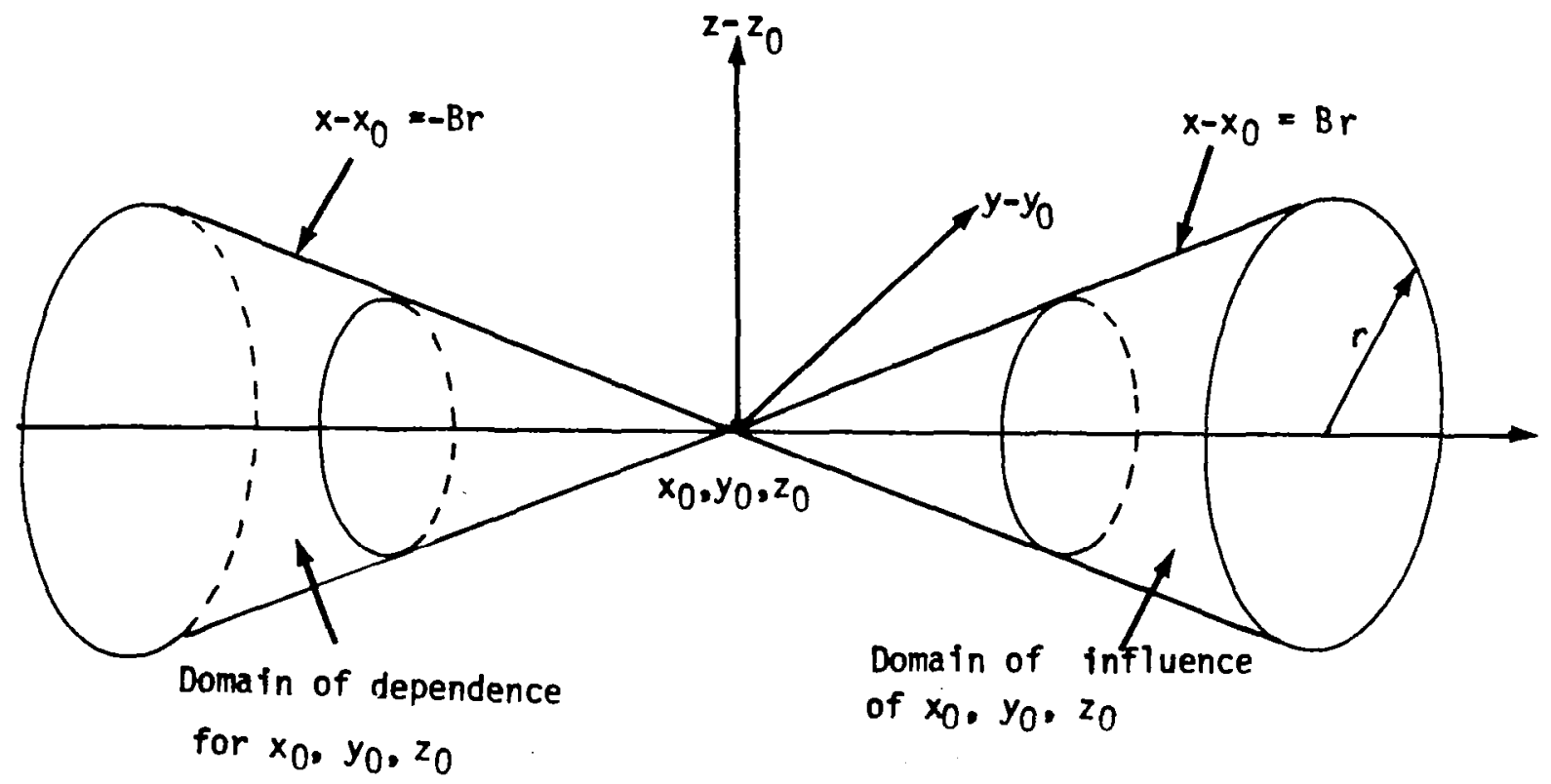

Figure 4.-Domains of Influence and Dependence of The Point $x_{0}, y_{0}, z_{0}$ in Supersonic Flow 


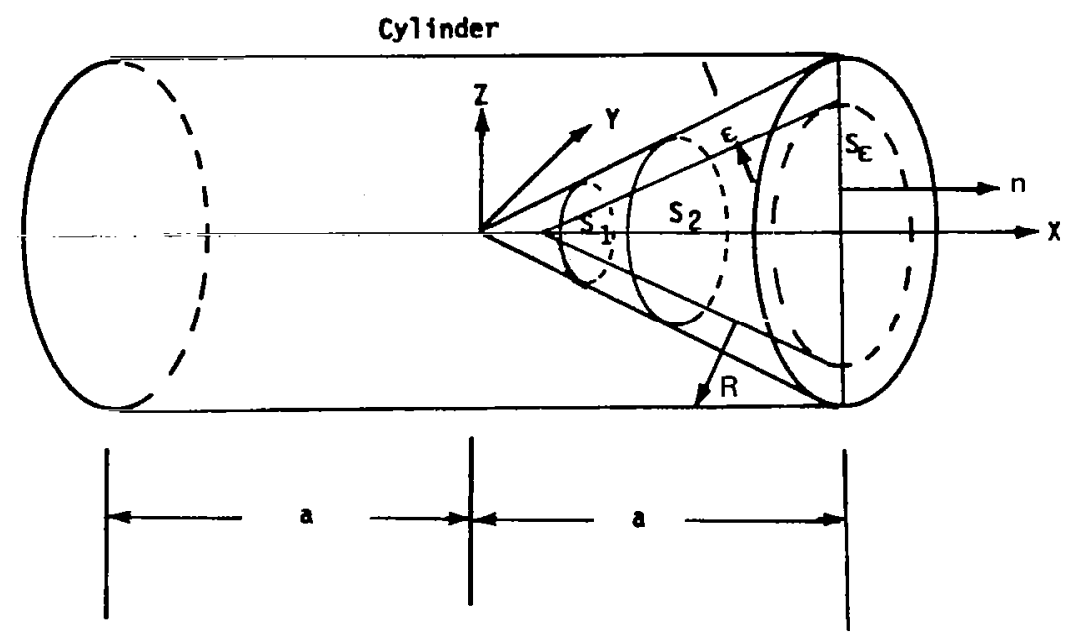

Figure 5.-Mass Flow Calculation For Supersonic Source

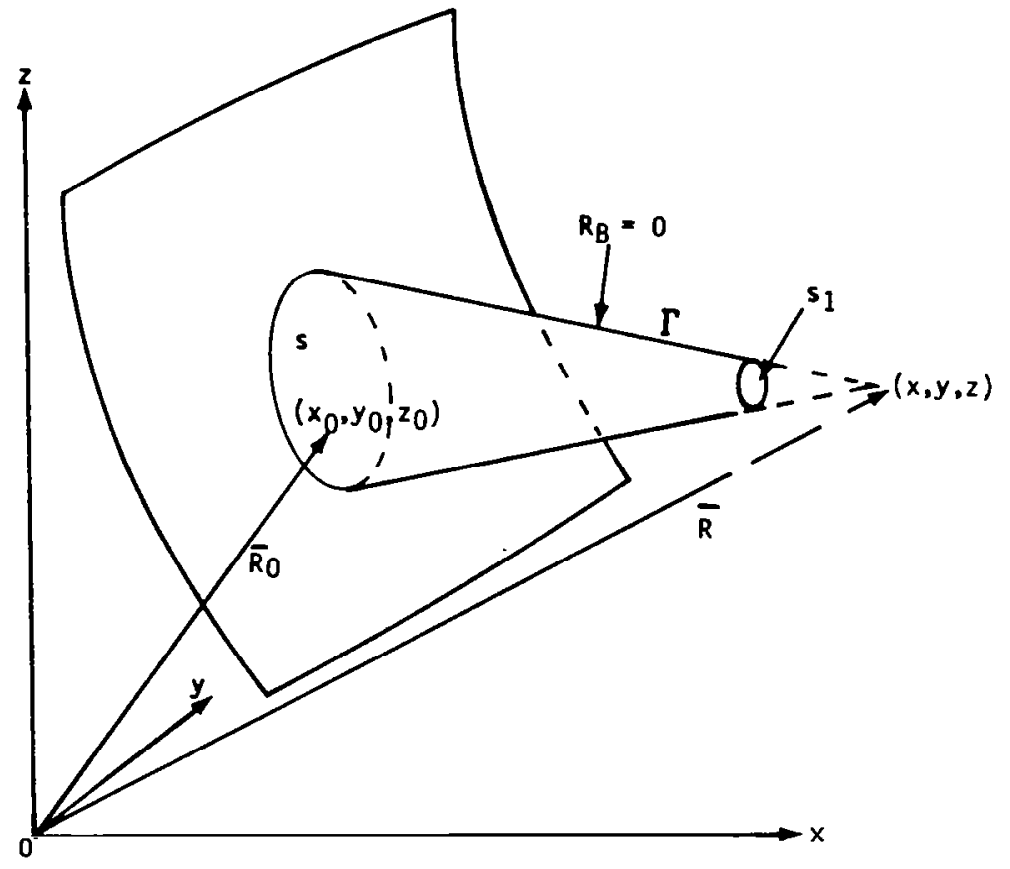

Figure 6.-Volume And Surfaces For Derivation of Integral Equation For Superinclined Surfaces in Supersonic Flow 


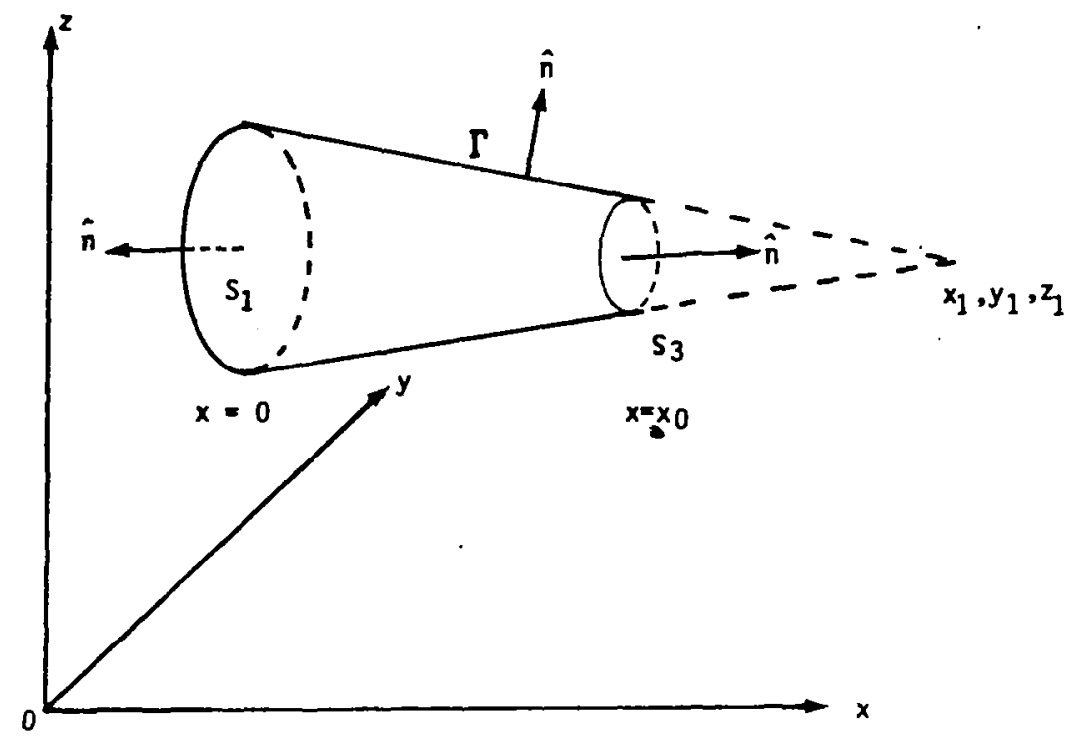

Figure 7.-Volume And Surfaces For The Uniqueness Proof From Boundary Conditions on Superinclined Surfaces in Supersonic Flow

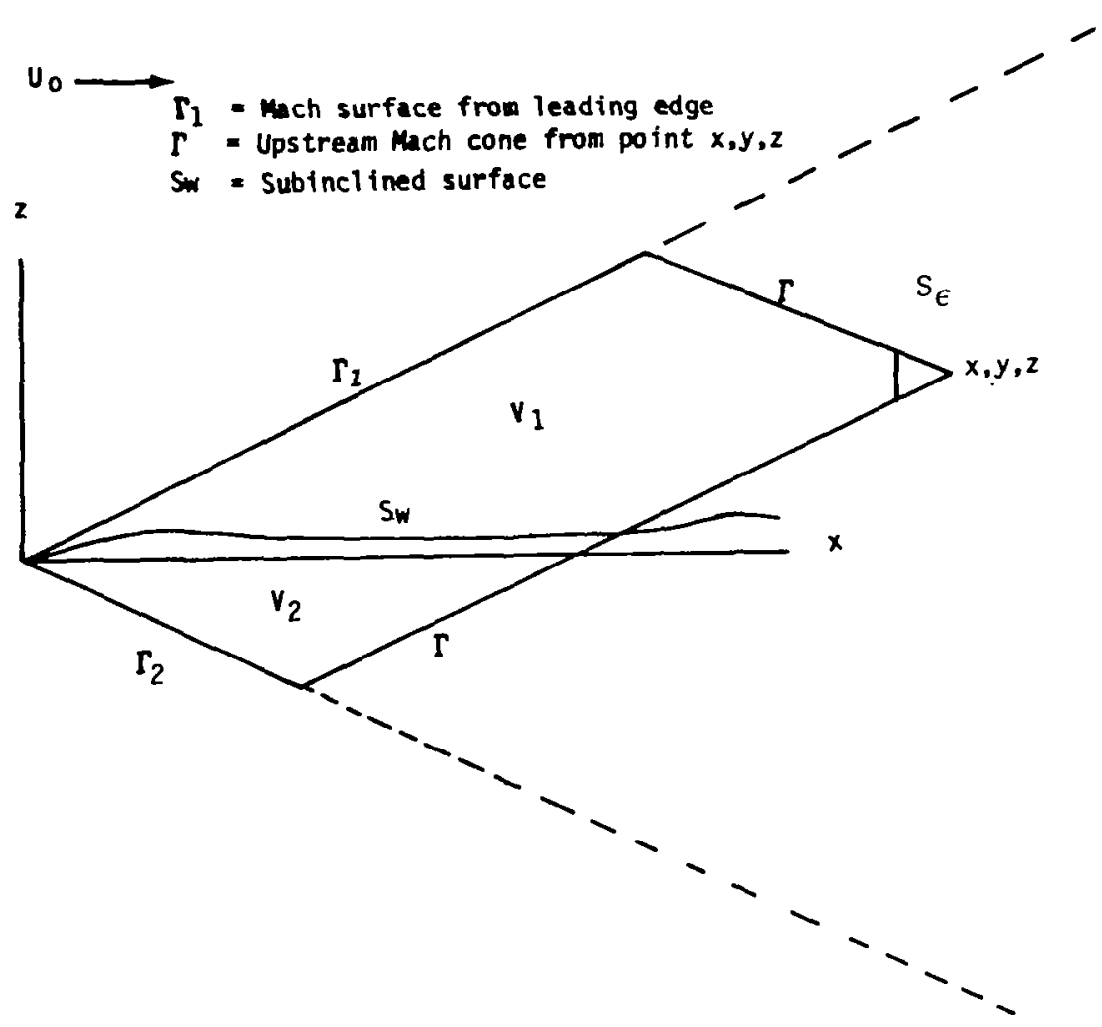

Figure 8. - Cross Section at $y_{0}=y$ of The Volumes and Surfaces For Deriving The Integral Equation For Subinclined Surfaces in Supersonic Flow 


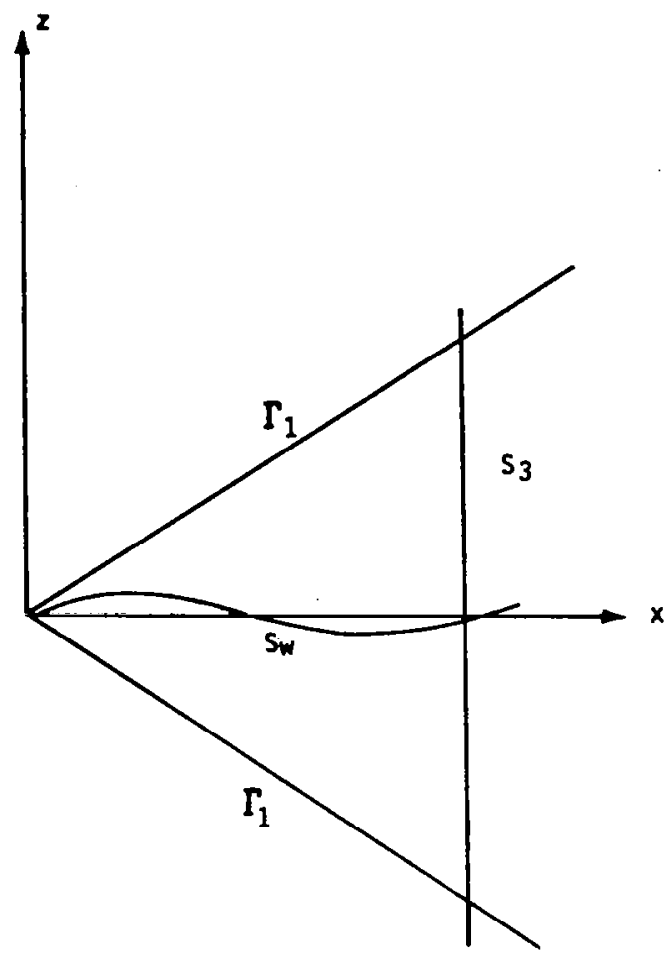

Figure 9.-Cross Section at $y=O$ of The Volumes And Surfaces For The Uniqueness Proof For Subinclined Surfaces in SuperSupersonic Flow

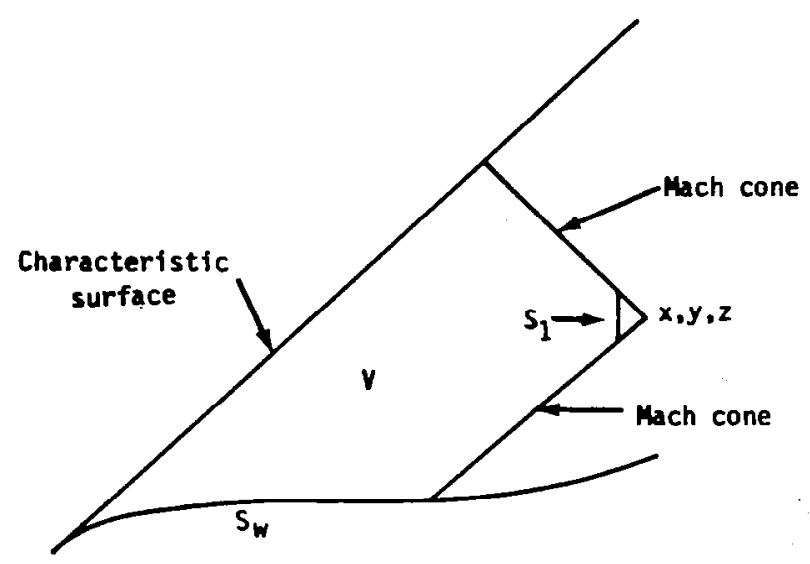

Figure 10.-Cross Section of Volume at $y=$ Constant And Surface For Calculating Velocity Neglecting Edge Vortices 


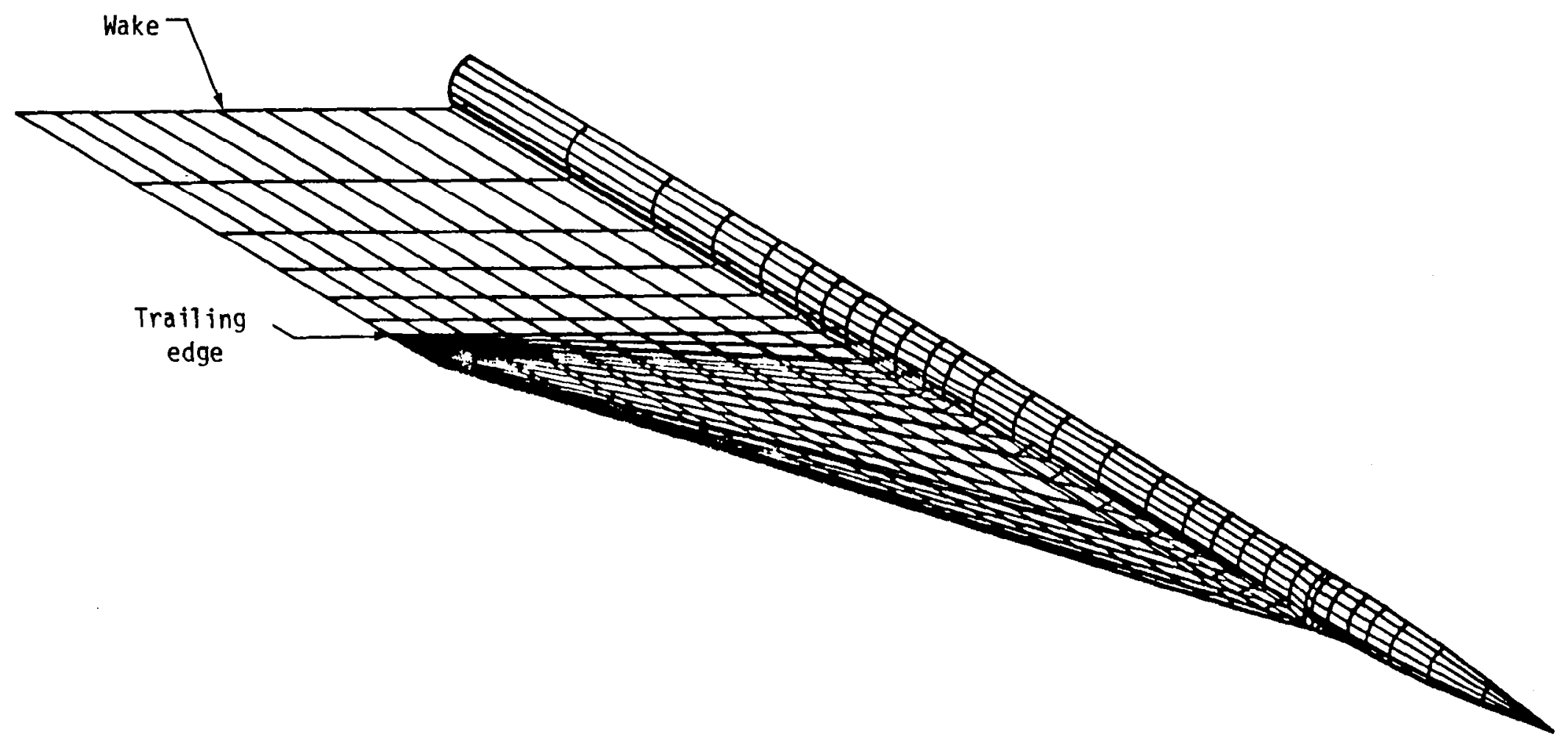

Figure 11.-Example of Wing and Body Paneling 


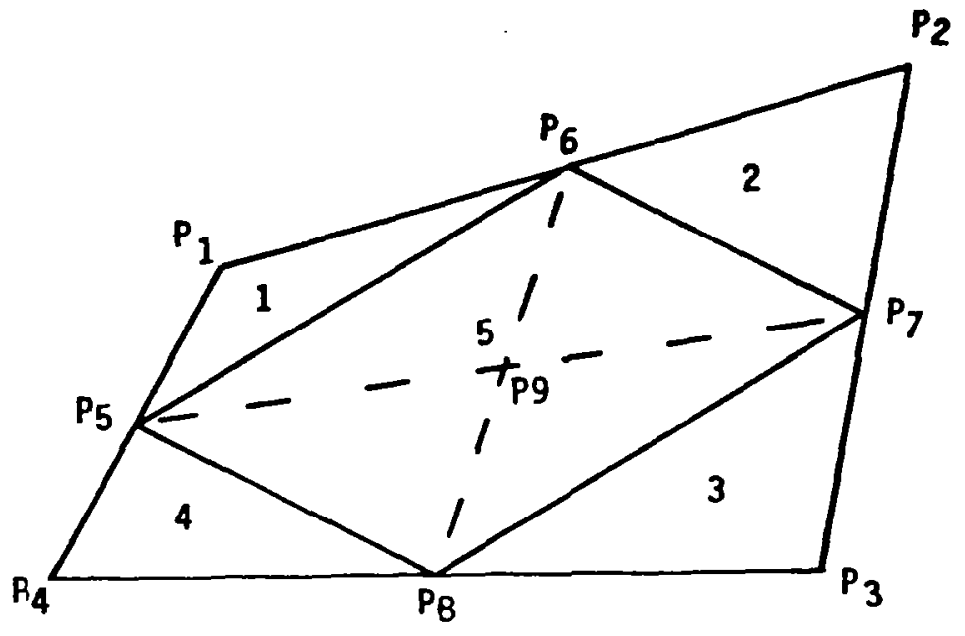

Figure 12.-Subpanel Structure of Basic Four Point Panel Showing The Five Planar Areas And Eight Triangular Subpanels 
Source/Analysis

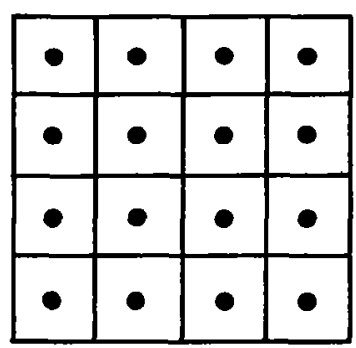

Figure 13.-Schematic Location of Control Points And Corresponding Values of Source Singularity Strength For Analysis Networks

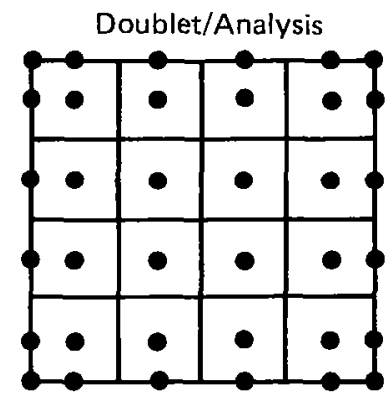

Figure 14.-Schematic Location of Control Points And Corresponding Values of Doublet Strength For Analysis Networks

(Doublet/Desiyn

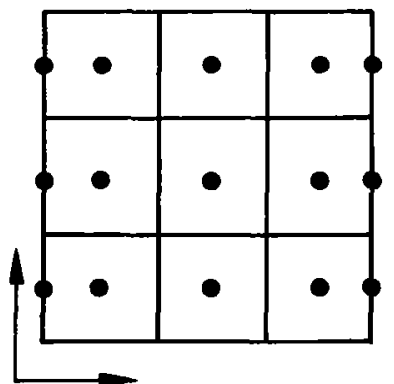

a. Control point locations

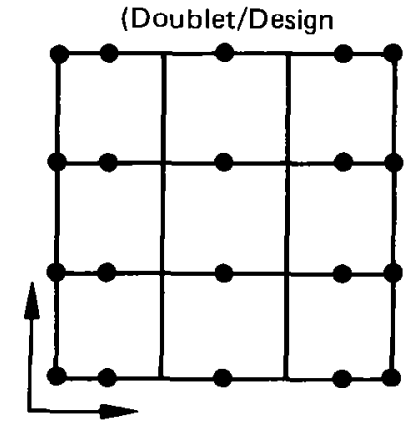

b. Singularity value locations

Figure 15.-Schematic Location of Control Points And Corresponding Values of Doublet Strength For Design Networks 


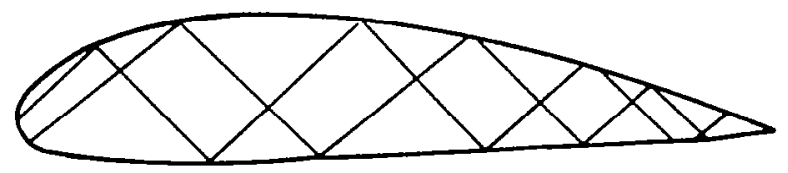

Figure 16. - Illustration of Mach Wave Propagation And Reflection of Interior Perturbations

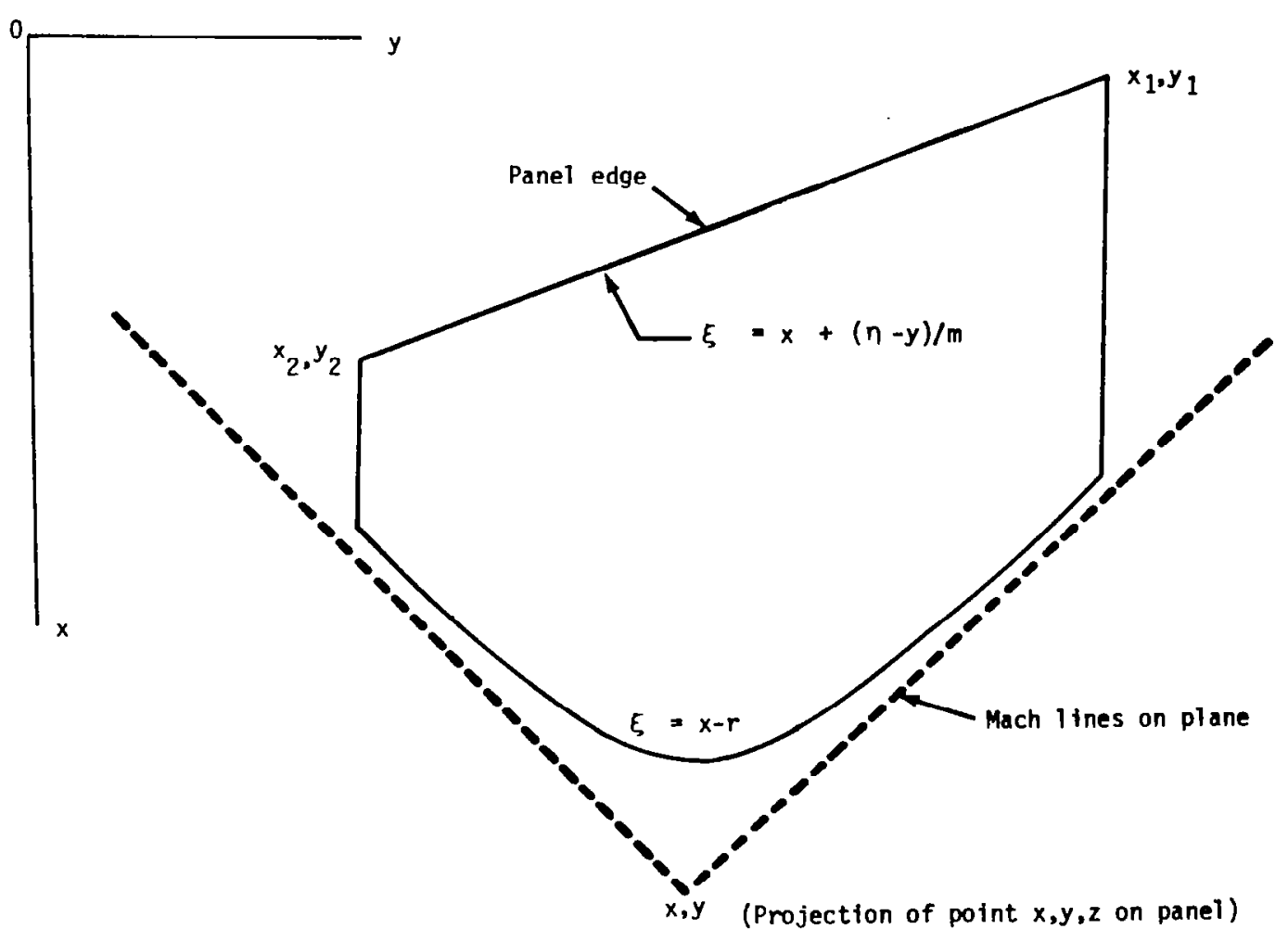

Figure 17.-Region of Integration For Subinclined Panel Edge 


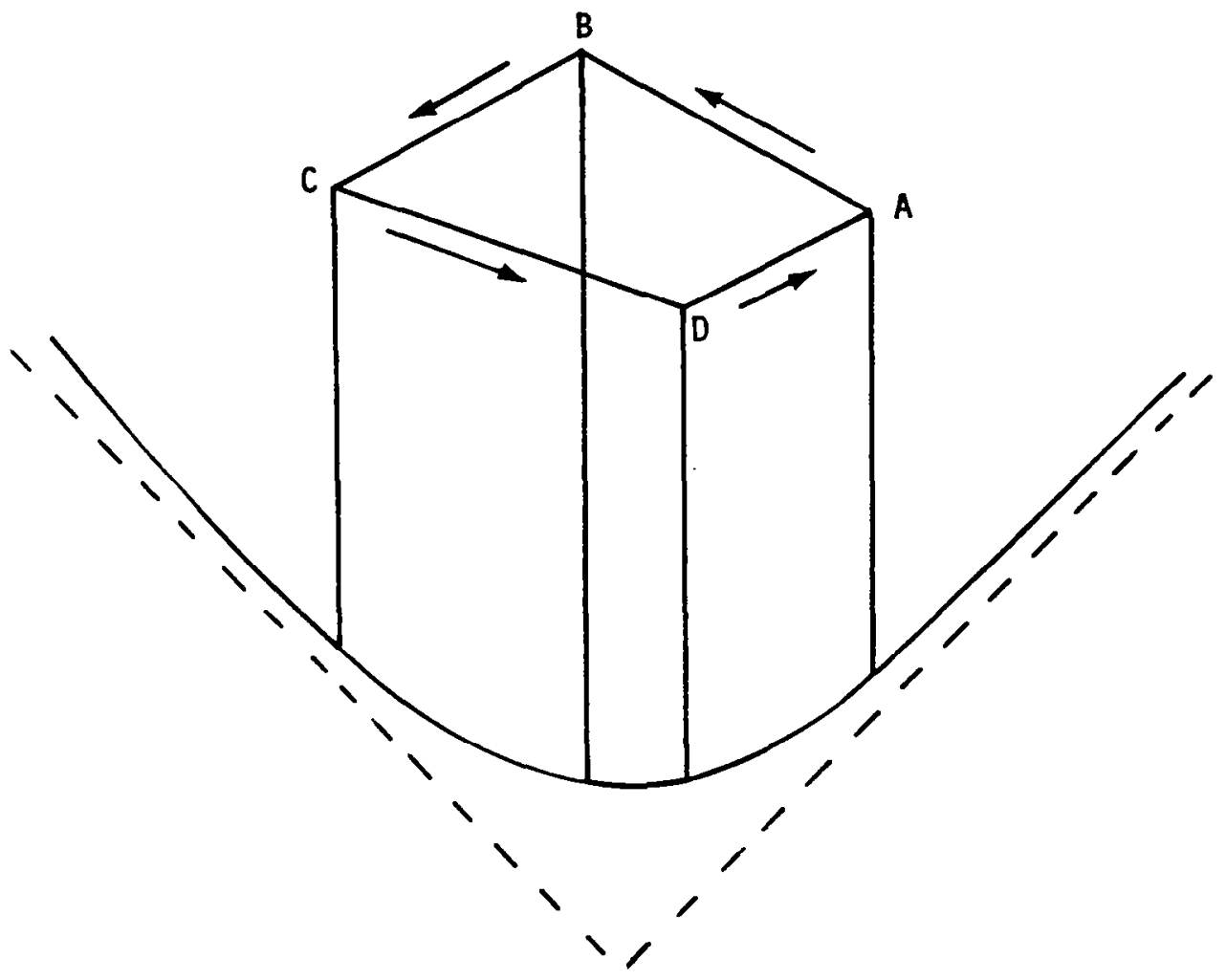

Figure 18.-IIlustration of Combining Edge Integrals to Find Integration Over a Panel 


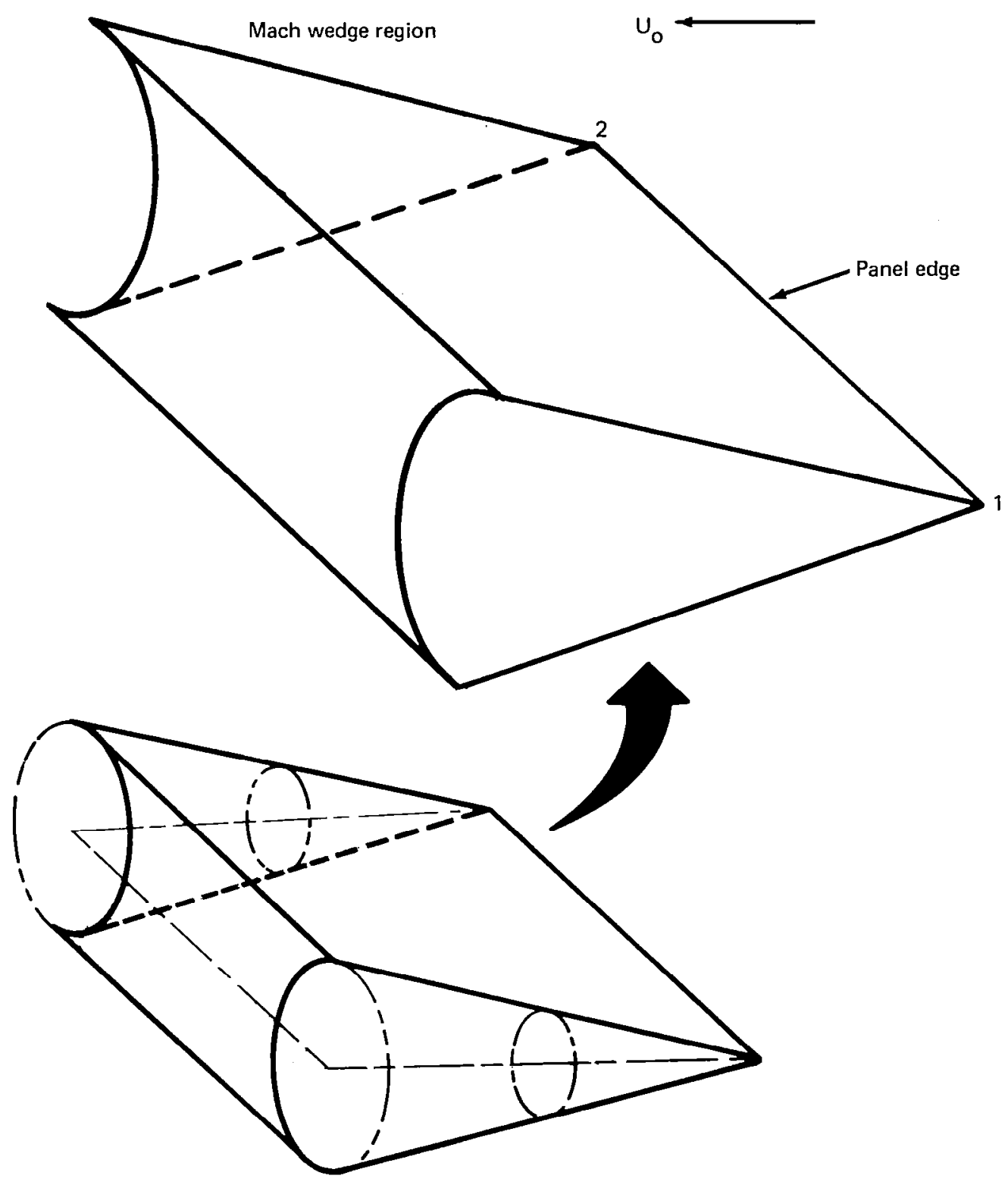

Figure 19.-Mach Wedge Region Behind Supersonic Panel Edge 


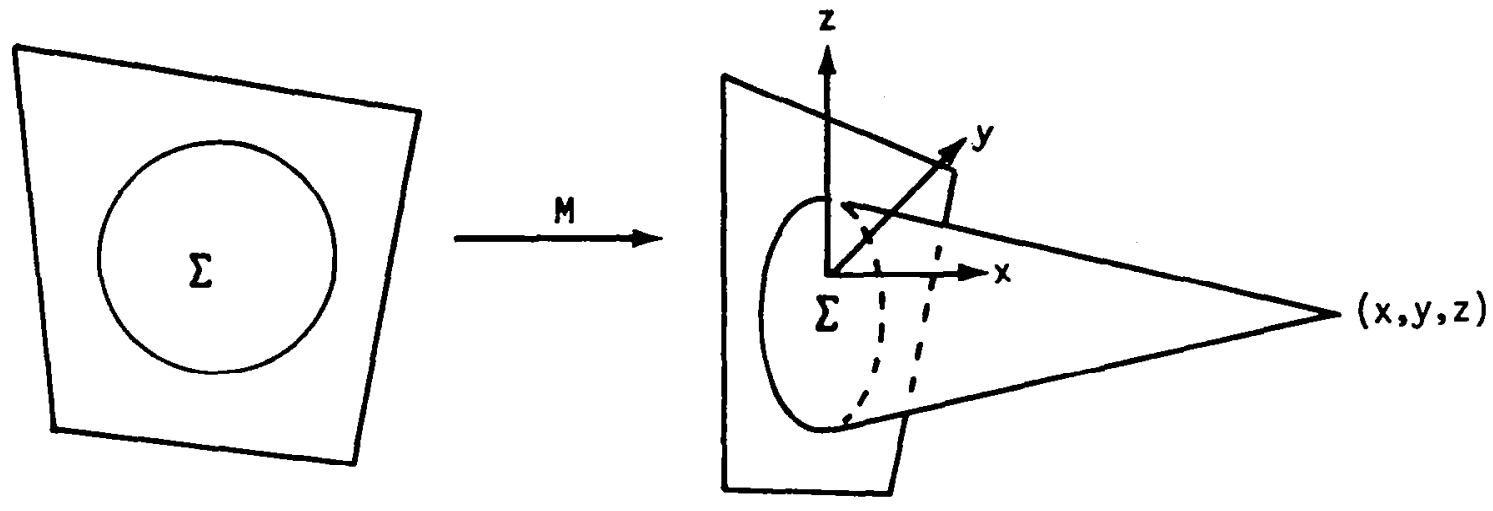

a. Mach cone intersection inside panel
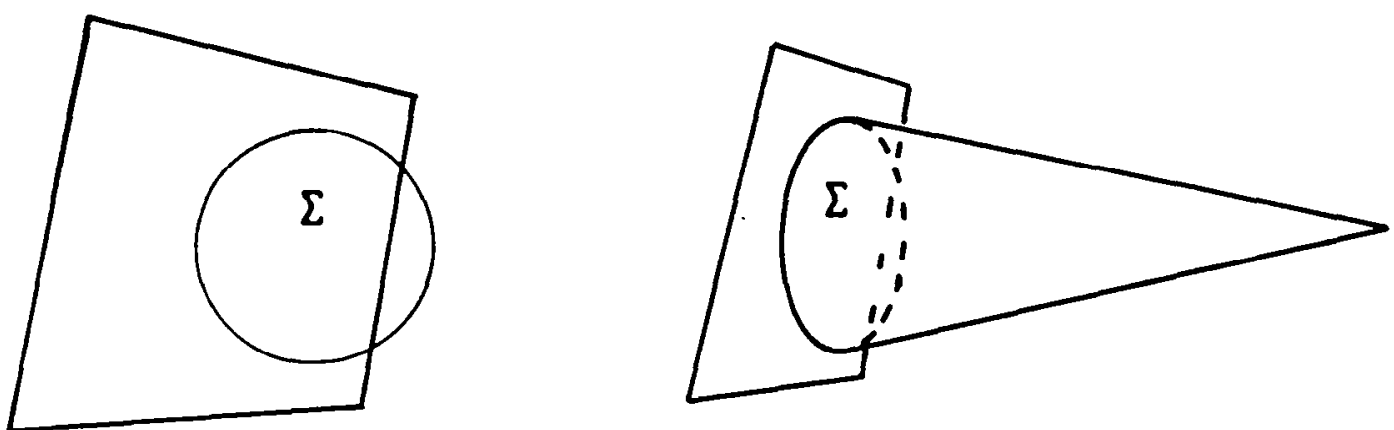

b. Mach cone intersecting one side of panel

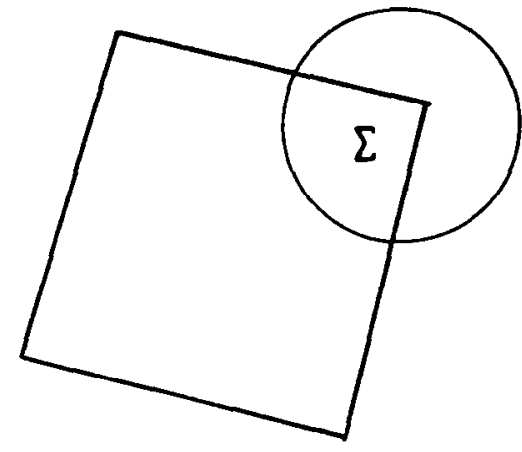

c. Mach cone intersecting panel corner

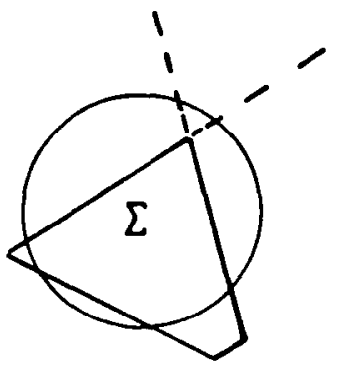

d. Mach cone intersecting panel corner cone and opposite side

Figure 20.-Examples of Mach Cone Intersections With Superinclined Panel Aligned Normal to The Free Stream 


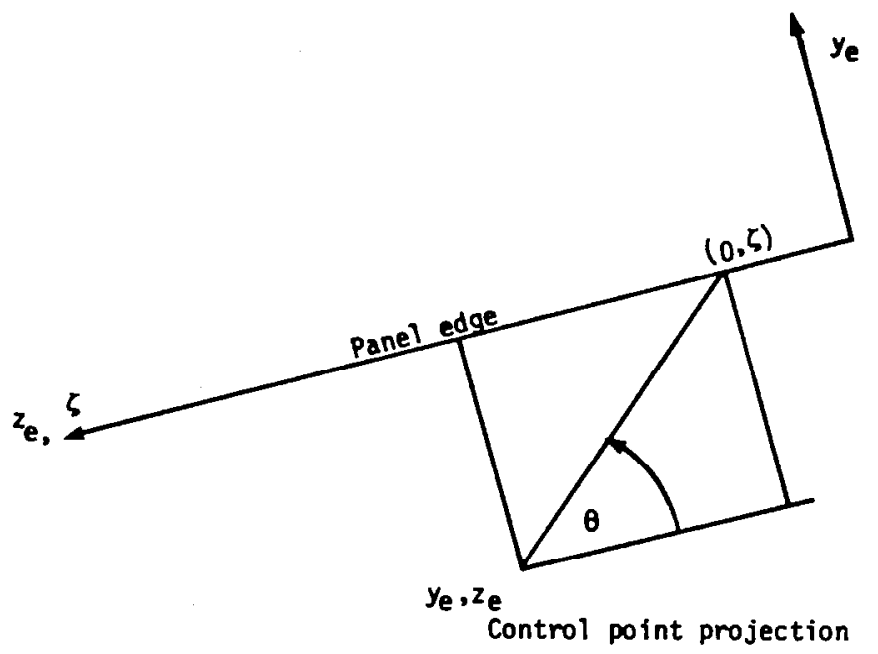

Figure 21.-IIlustration of Panel Edge Coordinates With Panel Edges Specified Counterclockwise on Exterior or Downstream Side of Superinclined Surface

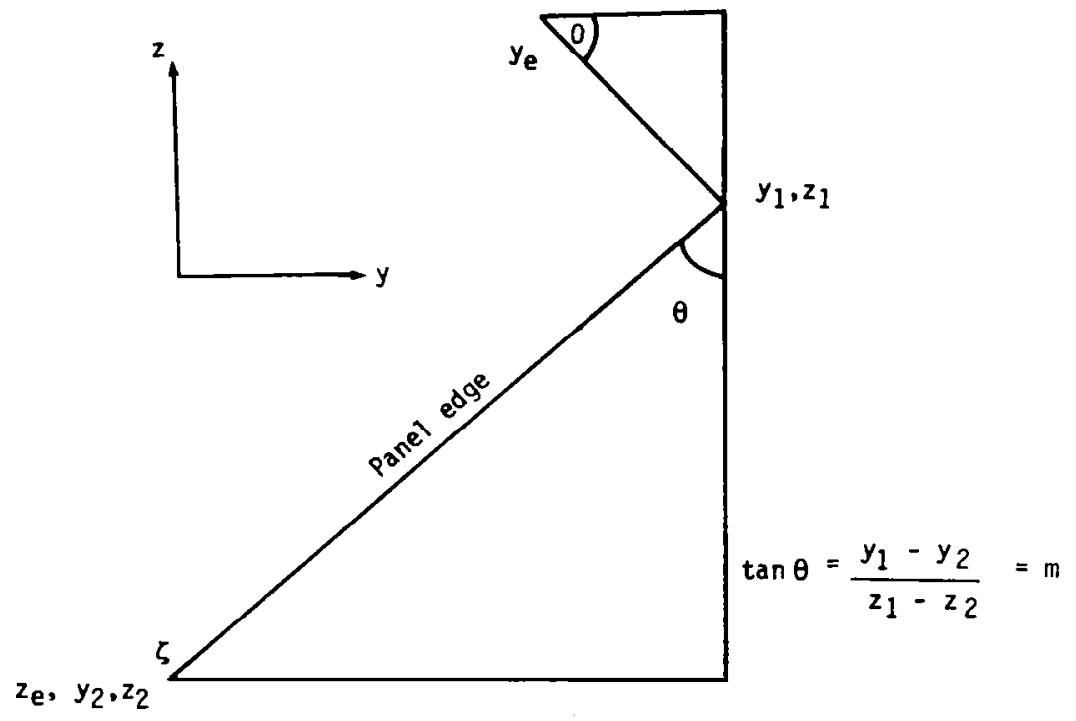

Figure 22.-Relation Between Panel And Edge Coordinate Systems of Superinclined Panel 


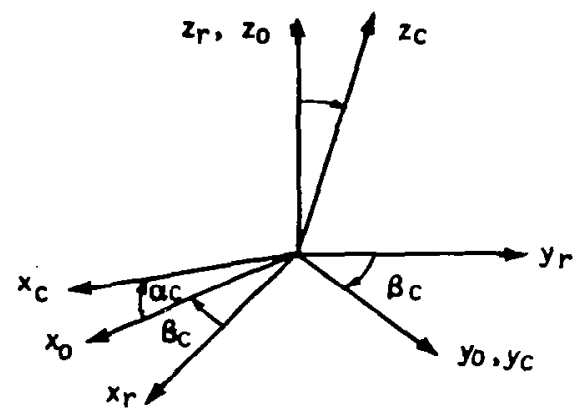

Figure 23. - Transformation From Reference Coordinates $x_{r}, y_{r}, z_{r}$ To Compressible Coordinates $x_{C}, y_{C^{\prime}} z_{C}$

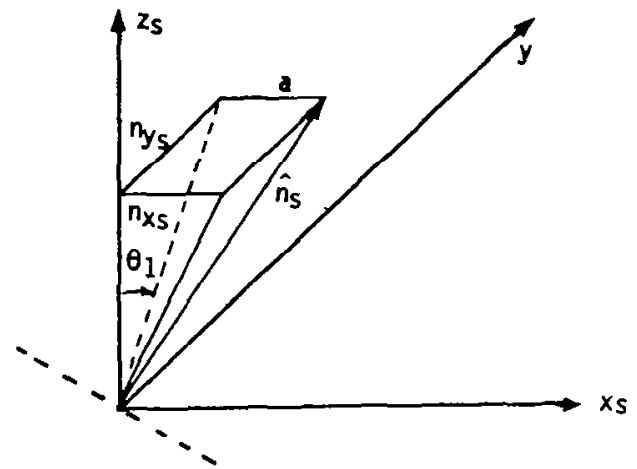

Figure 24. - First Angle of Rotation For Panel Coordinate System

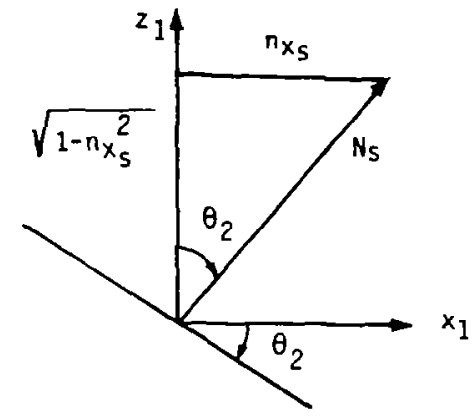

Figure 25.-IIlustration of Second Angle of Rotation For Subsonic Flow or of Oblique Transformation For Supersonic Flow 


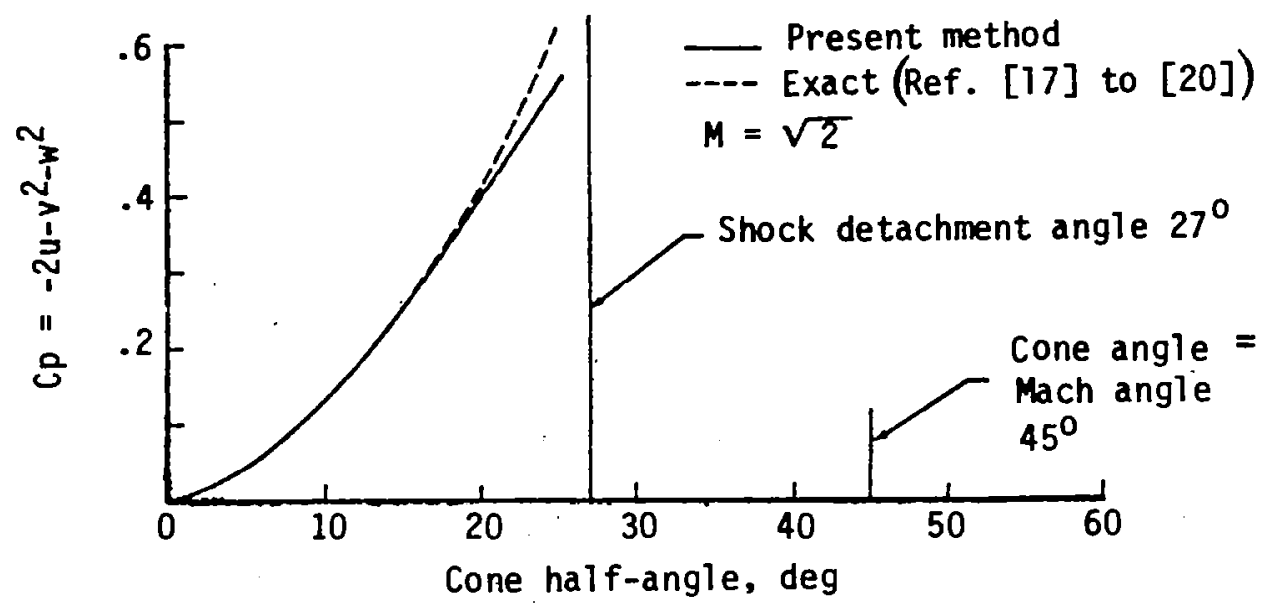

Figure 26.-Comparison of Pressure Coefficient on Cones at Zero Angle of Attack With Exact Theory

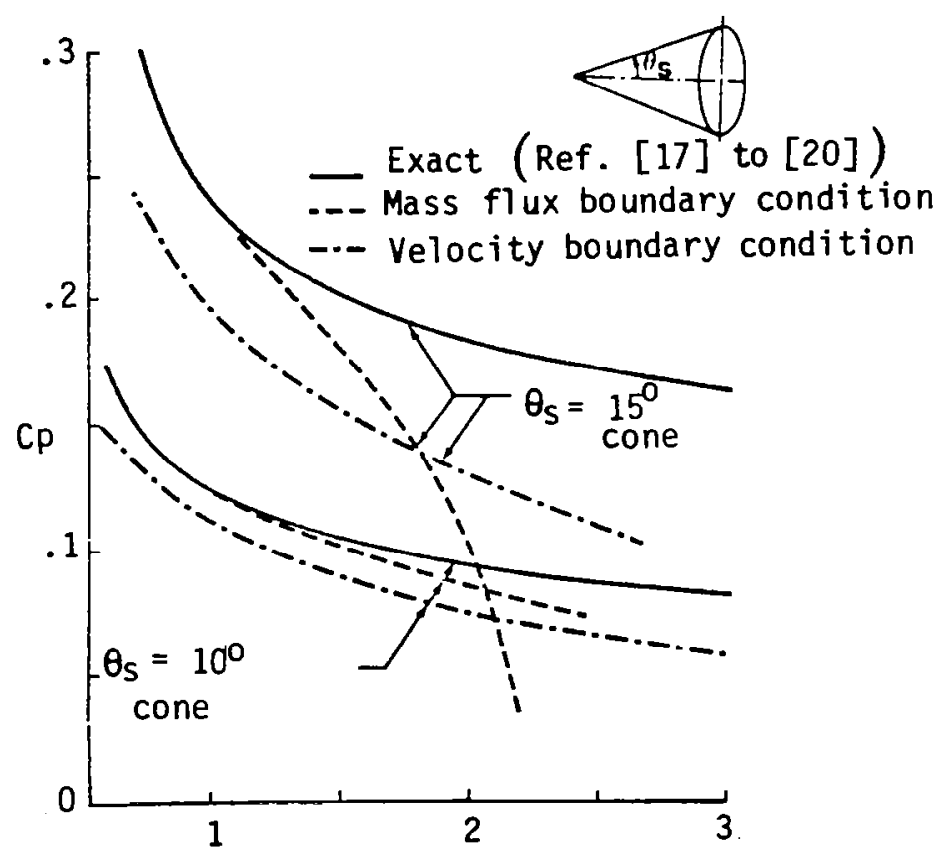

Figure 27.-Comparison of Three Methods for Computing Pressure Coefficients on $10^{\circ}$ And $15^{\circ} \mathrm{Half}$-angle Cones at Zero Angle of Attack as a Function of Mach Number 


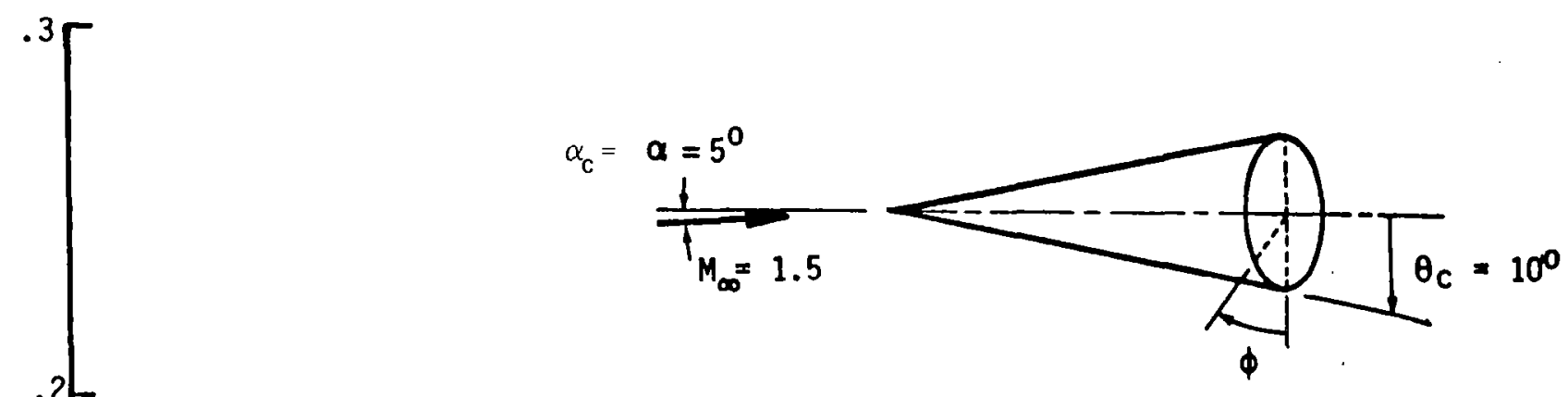

$c_{p}$
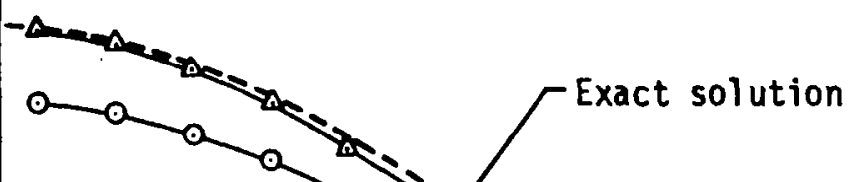

$\angle$ Mass flux boundary conditions

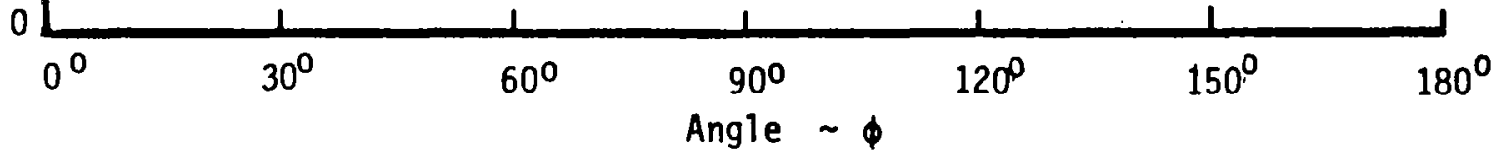

Figure 28.-Comparison of Pressure Coefficients Computed by Zero Normal Mass Flux Boundary Conditions And Zero Normal Velocity Boundary Conditions 

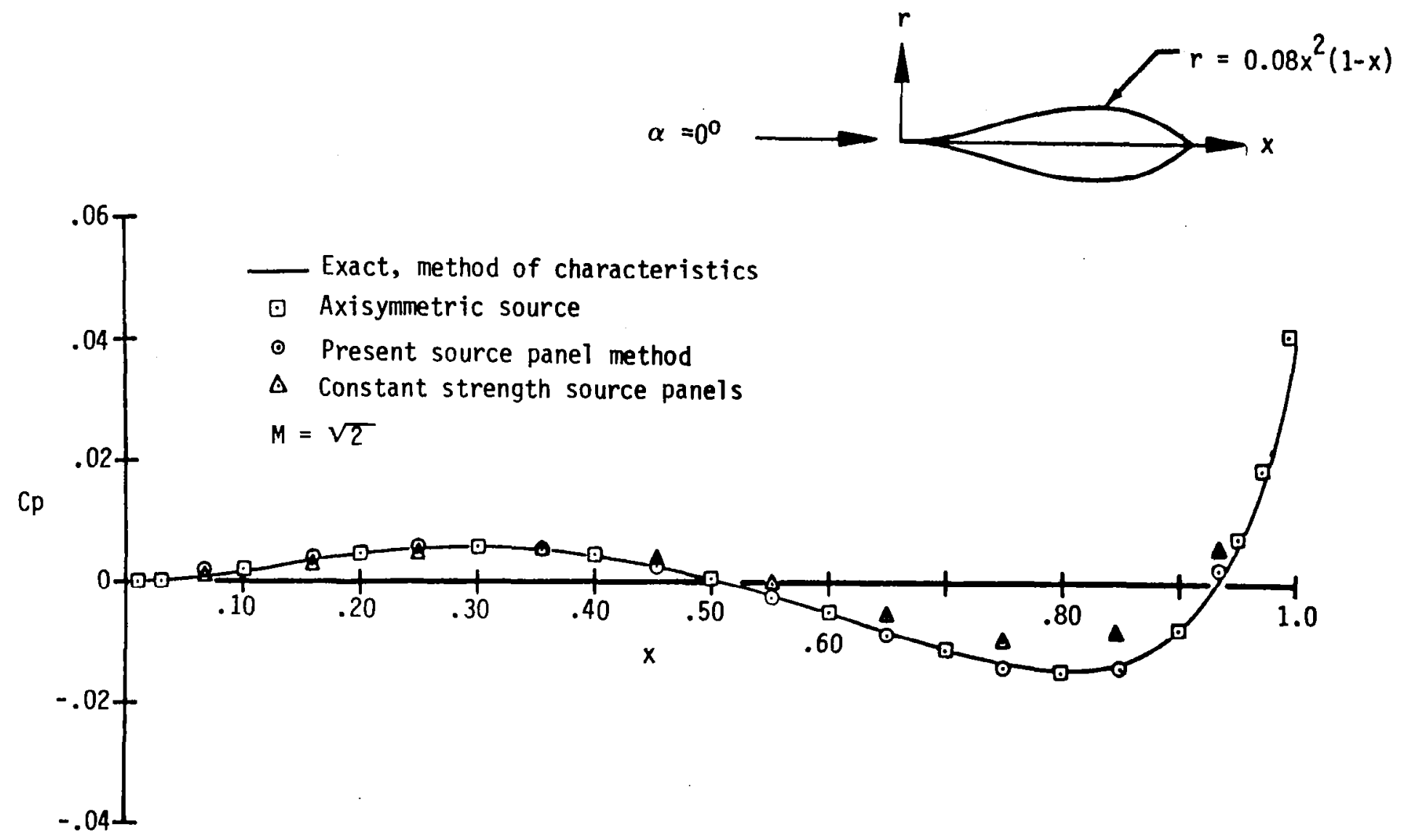

Figure 29. - Comparison of Four Methods For Computing The Pressure Coefficient on The Spindle $r=0.08 x^{2}(1-x)$ 


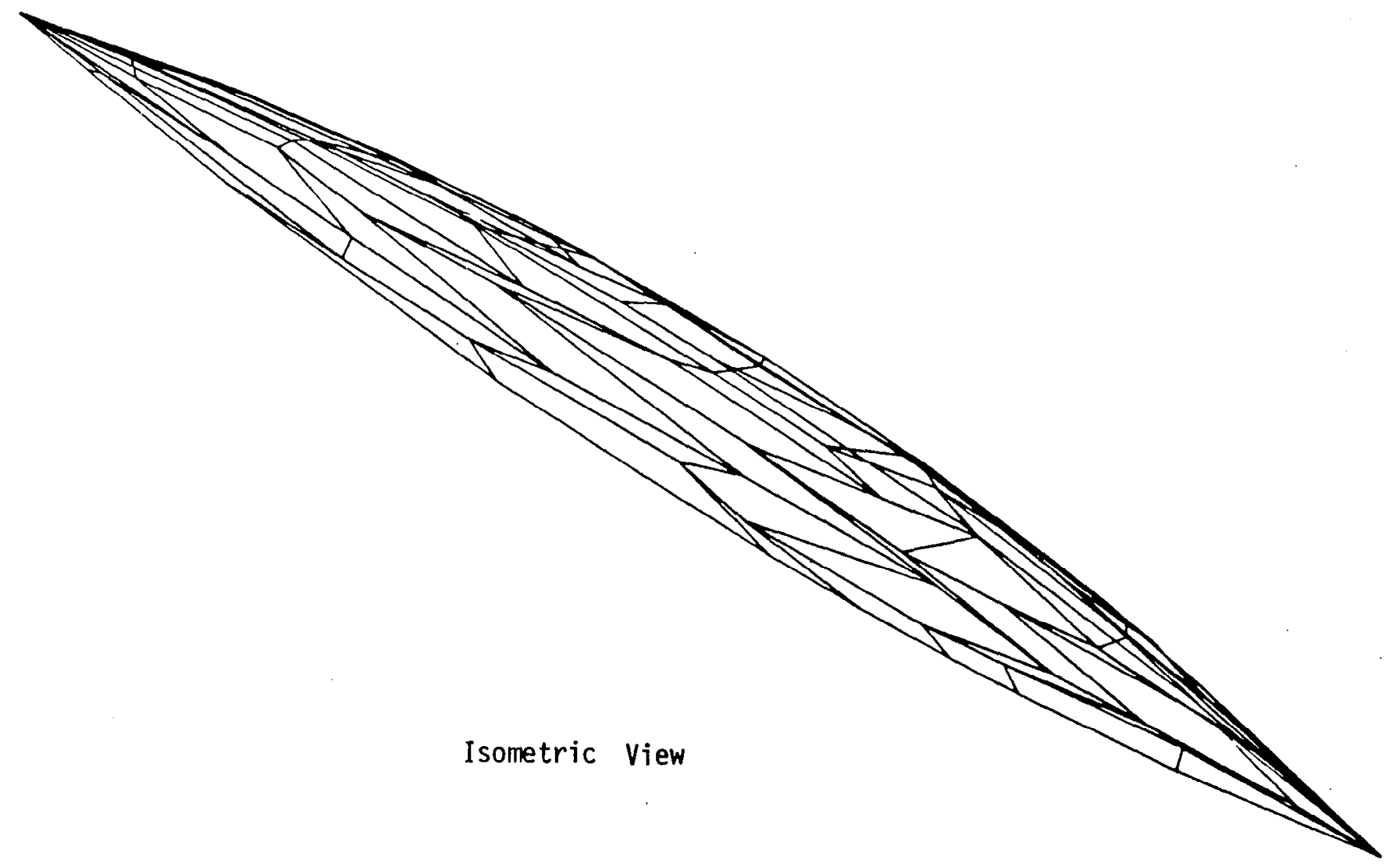

Figure 30. - Random Paneling on the Spindle $r=x(1-x) / 5$ Chosen to Test Stability of Source Panel Method 


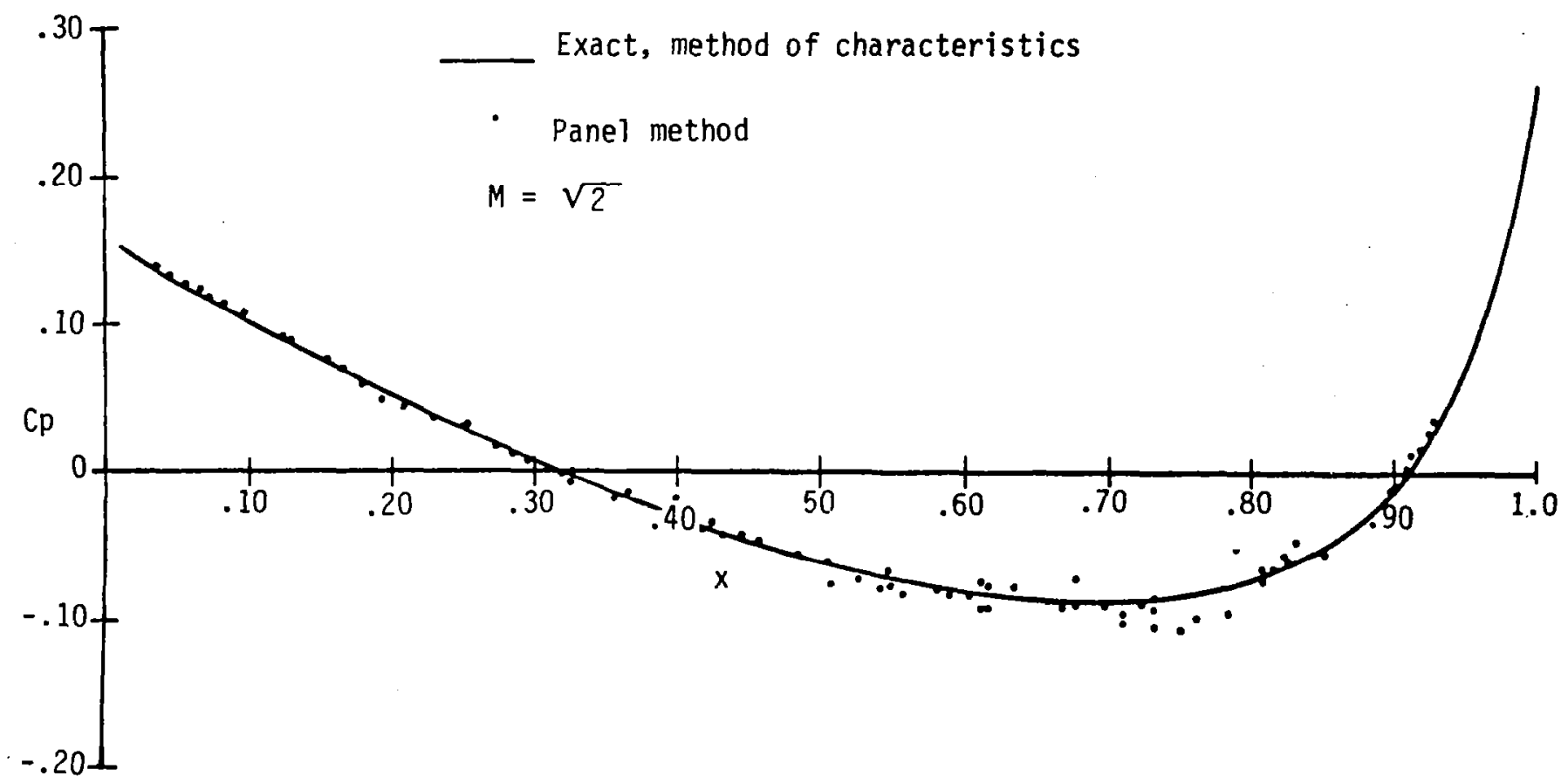

Figure 31. - Pressure Distribution From Random Paneling on The Spindle $r=x(1-x) / 5$ at Zero Angle of Attack 


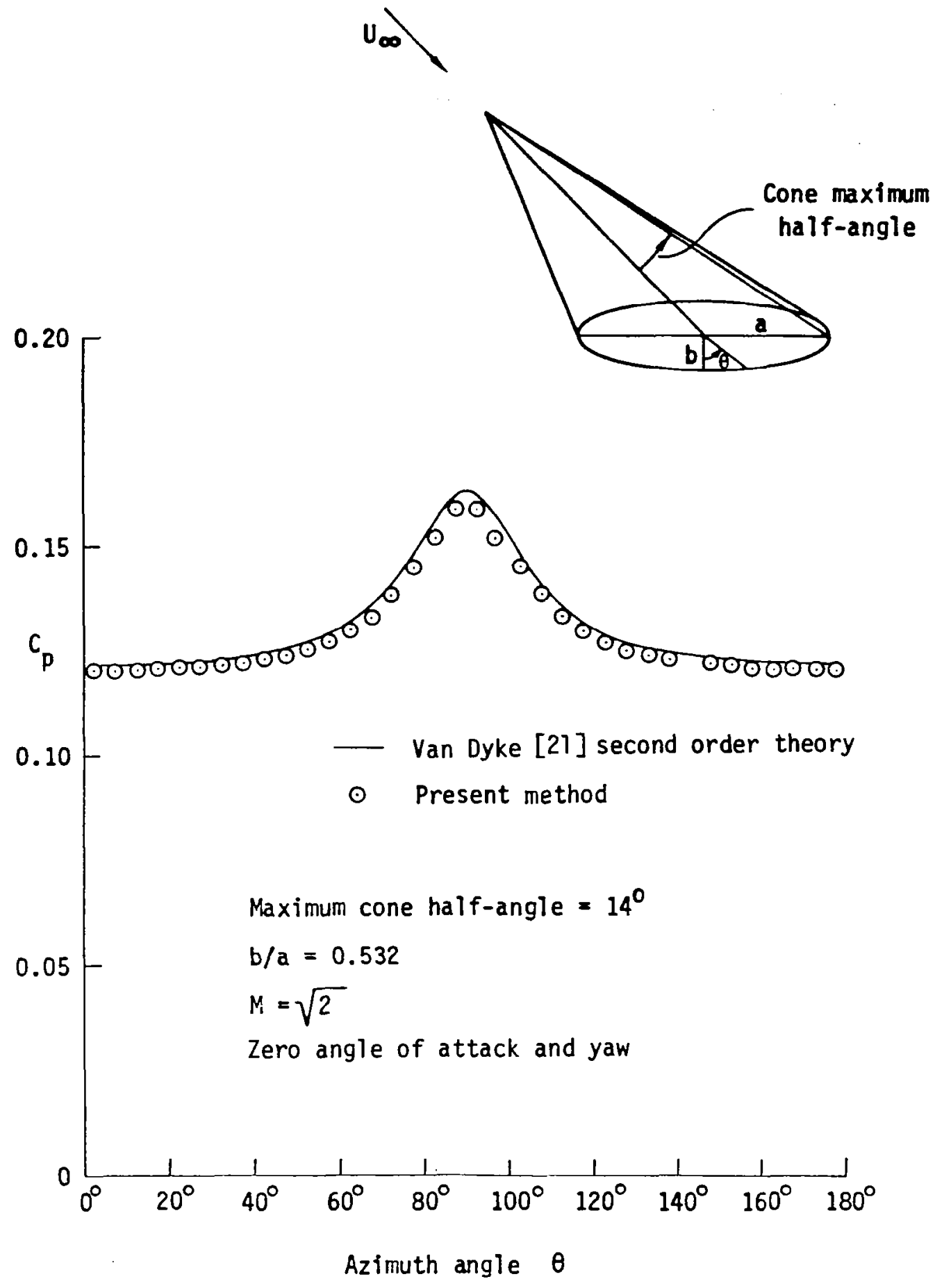

Figure 32.-Comparison of The Pressure Distribution by The Present Method With The Van Dyke Second Order Theory For The Elliptic Cone Having a Maximum Half Angle of $14^{\circ}$ And Fineness Ratio of 0.532 

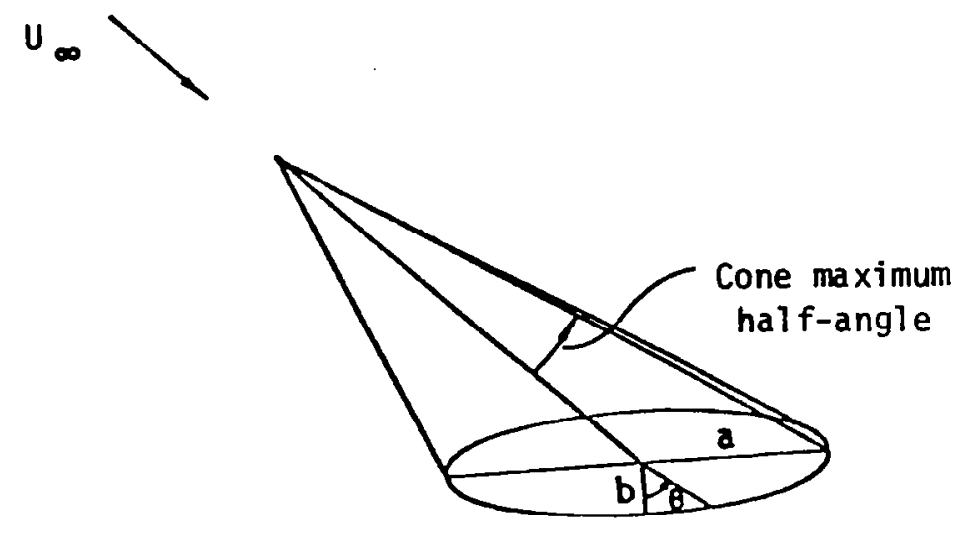

Van Dyke [21] second order theory.

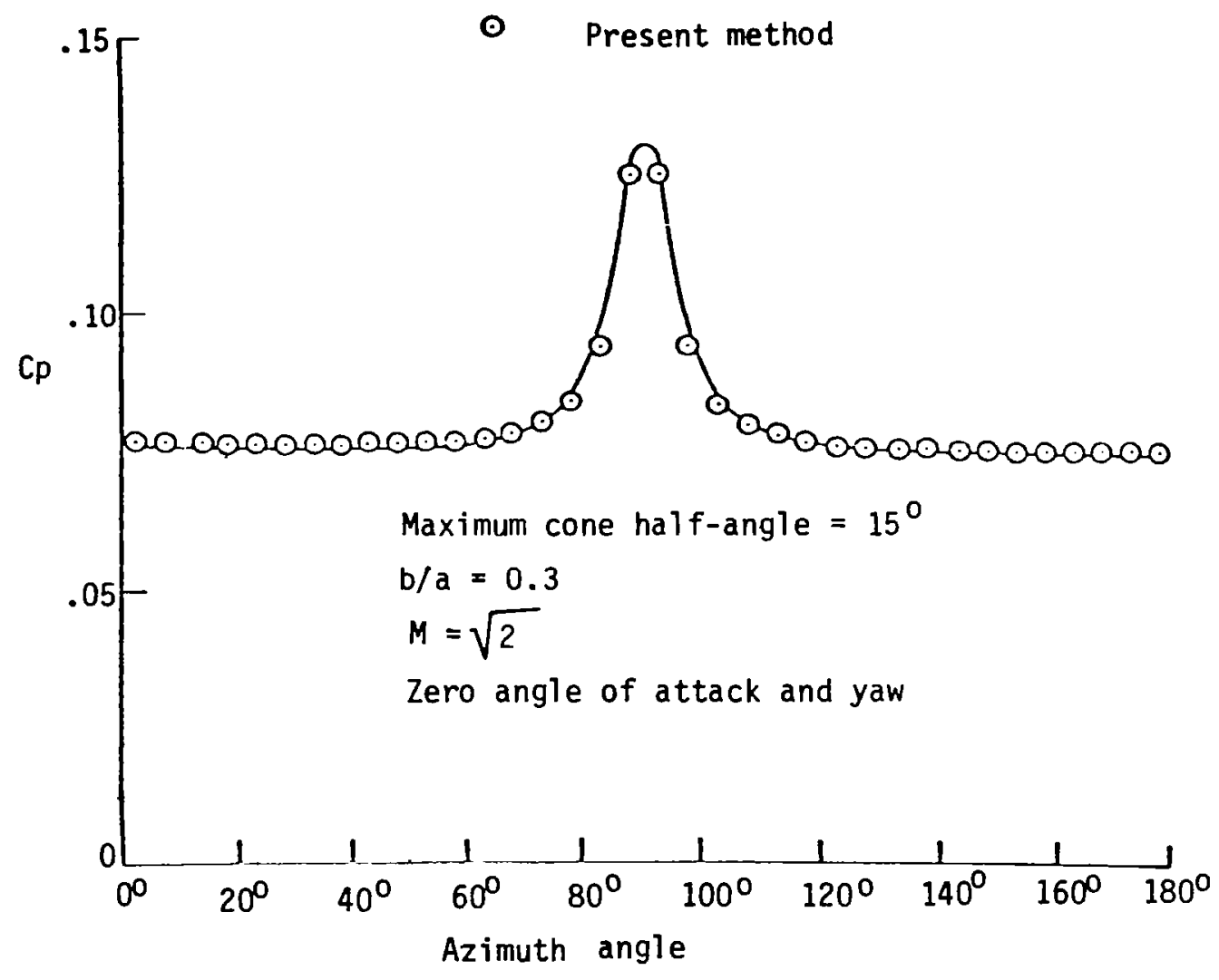

Figure 33. - Comparison of The Pressure Distribution by The Present Method With The Van Dyke Second Order Theory For The Elliptic Cone Having a Maximum Half Angle of $15^{\circ}$ And a Fineness Ratio of 0.3 


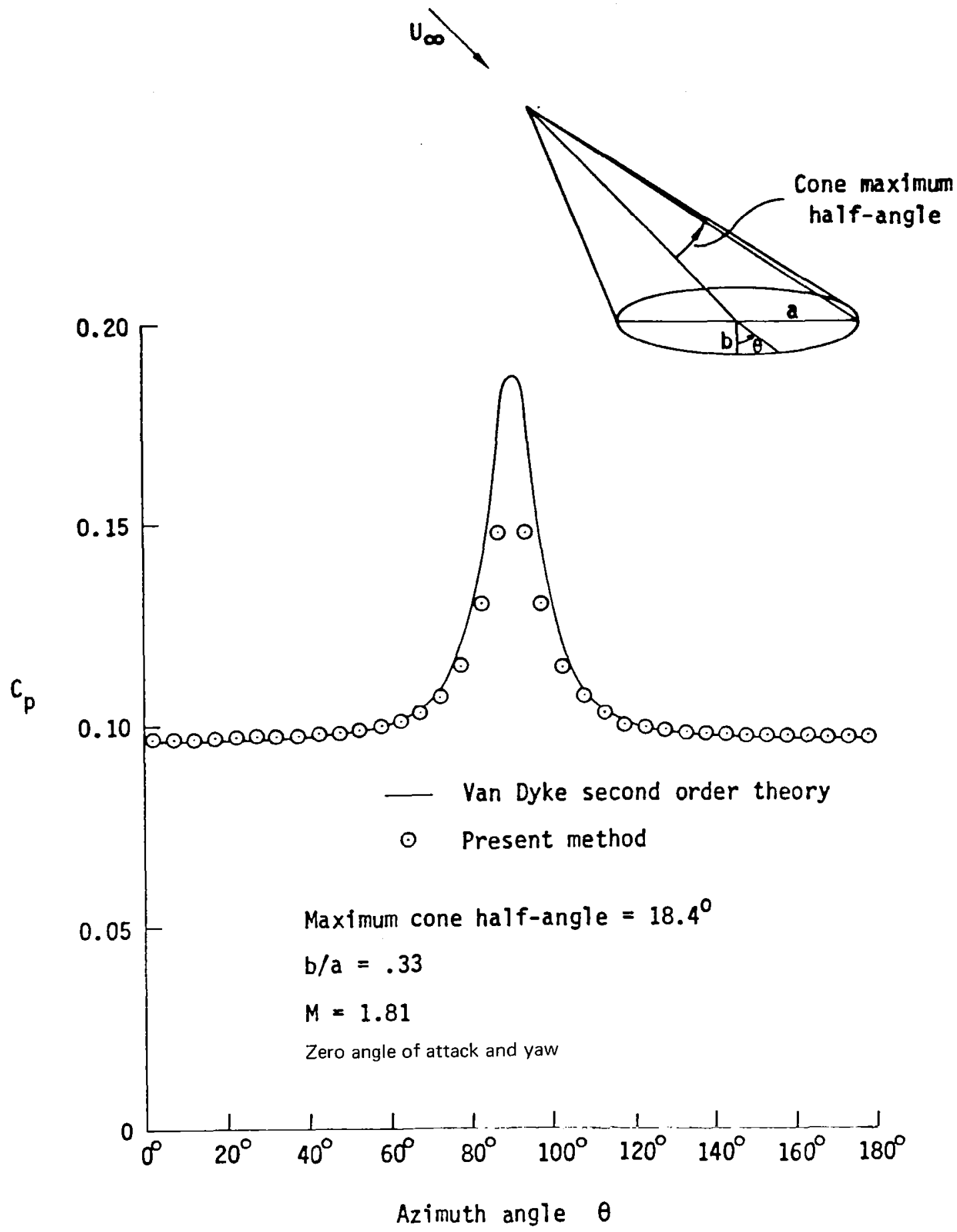

Figure 34.-Comparison of The Pressure Distribution by The Present Method With The Van Dyke Second Order Theory For The Elliptic Cone Having a Maximum Half Angle of $18.4^{\circ}$ And a Fineness Ratio of 0.33 


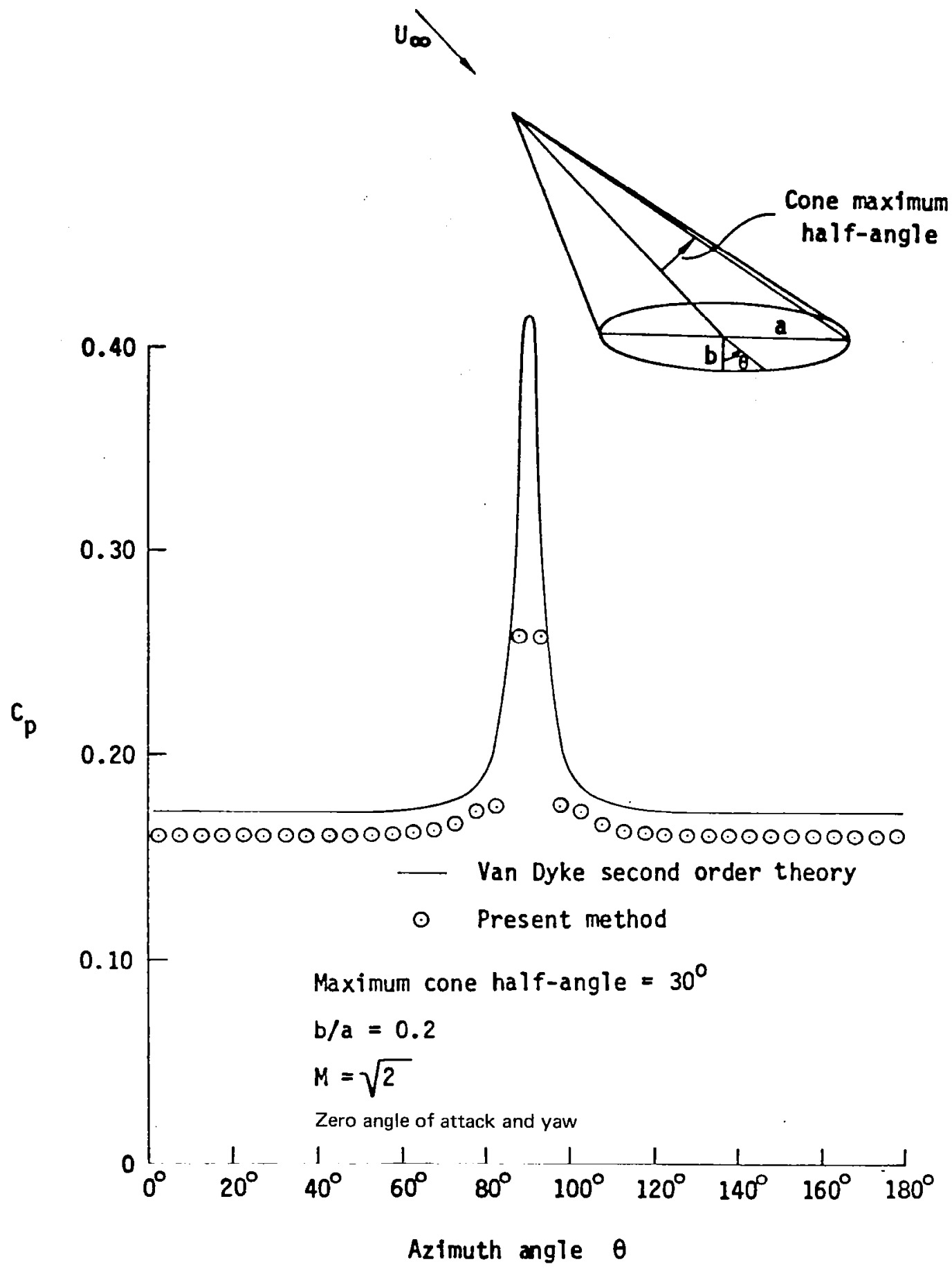

Figure 35.-Comparison of The Pressure Distribution by The Present Method With The Van Dyke Second Order Theory For The Elliptic Cone Having a Maximum Half Angle of $30^{\circ}$ And a Fineness Ratio of 0.2 


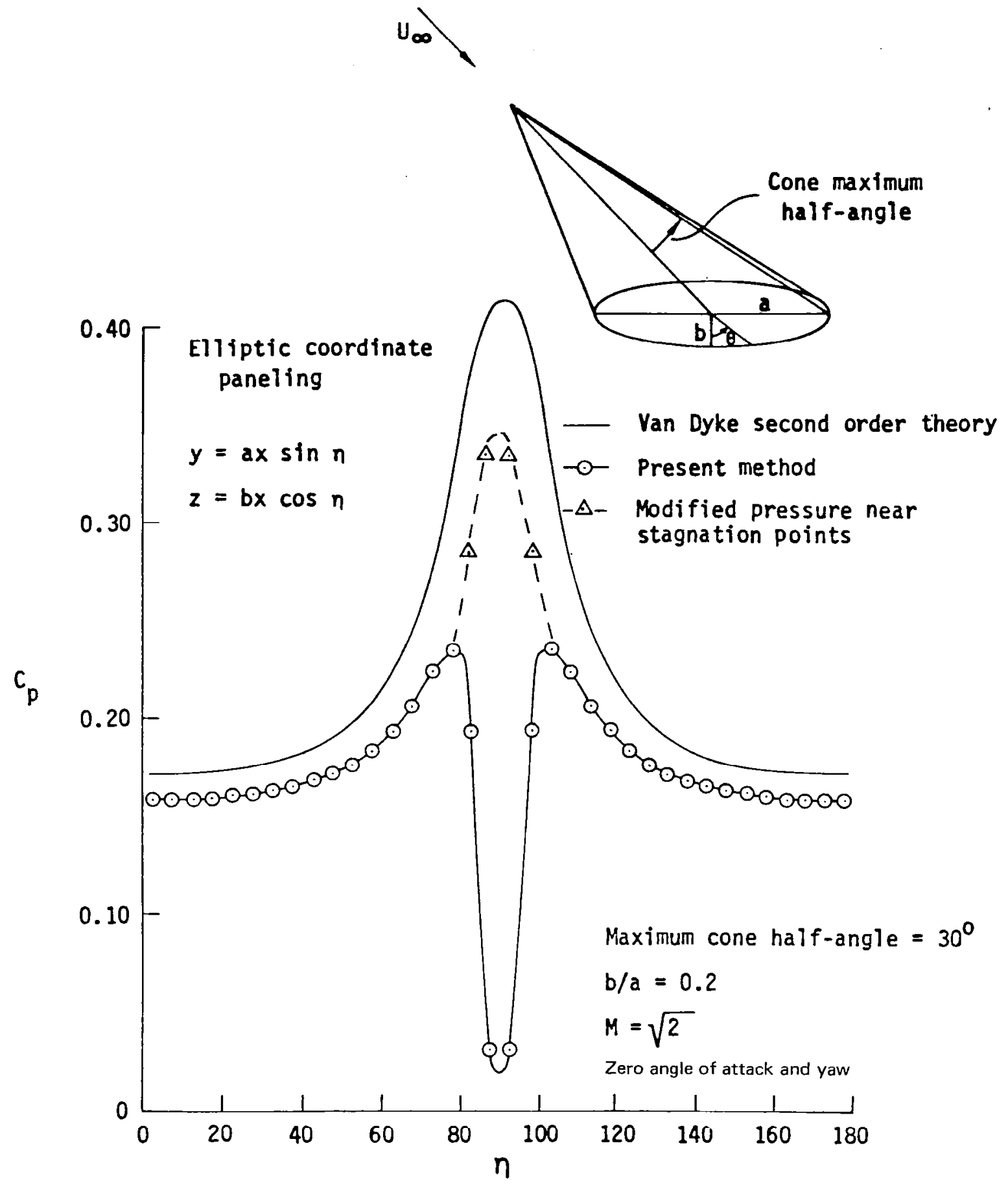

Figure 36.-Comparison of The Pressure Distribution by The Present Method With The Van Dyke Second Order Theory For The Elliptic Cone Having a Maximum Half Angle of $30^{\circ}$ And Fineness Ratio of 0.2 With Refined Paneling And Modified Pressure Calculation For Near Stagnation Conditions 


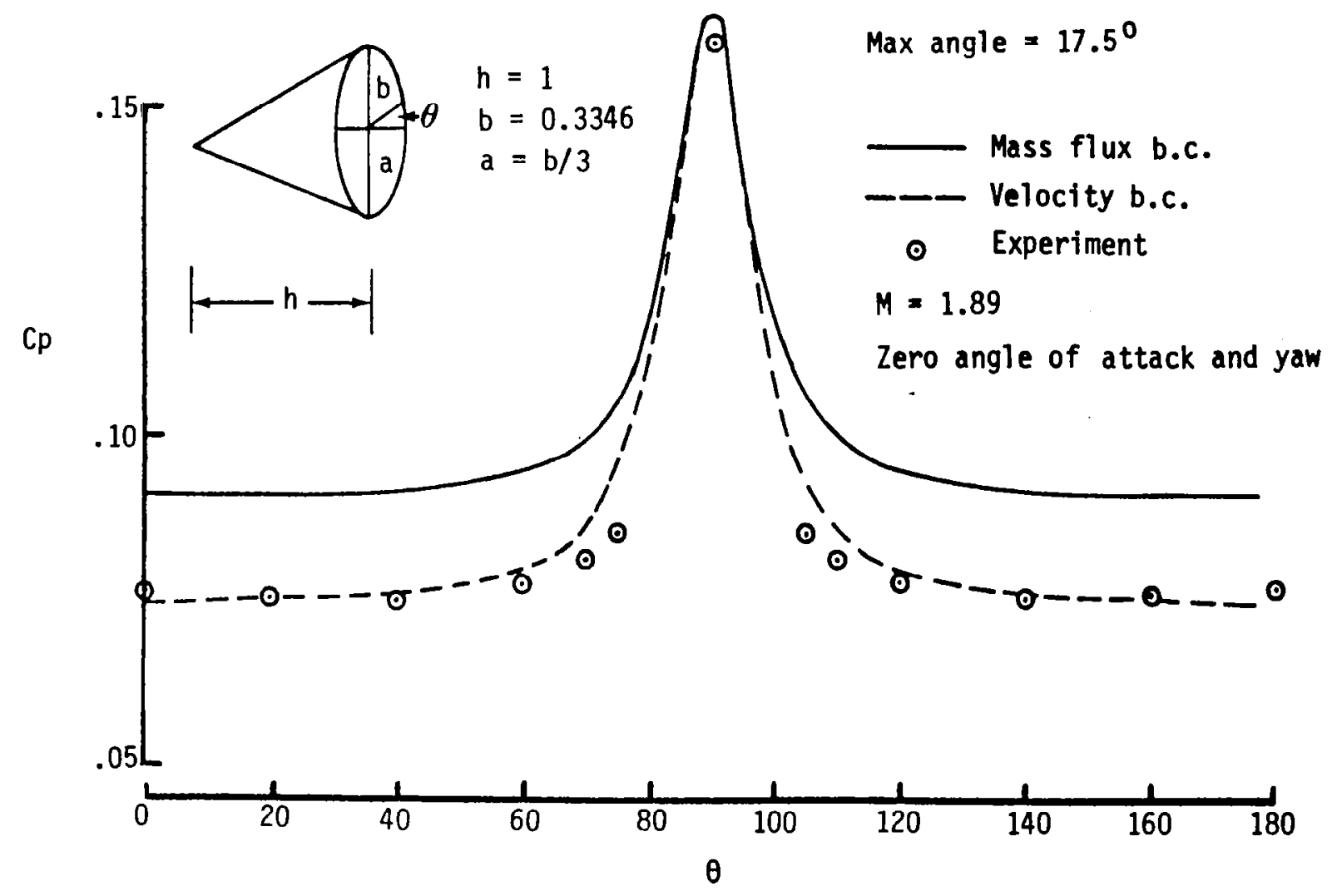

Figure 37.-Comparison of Pressure Distributions on an Elliptic Cone Resulting From Mass Flux and Velocity Type Boundary Conditions 


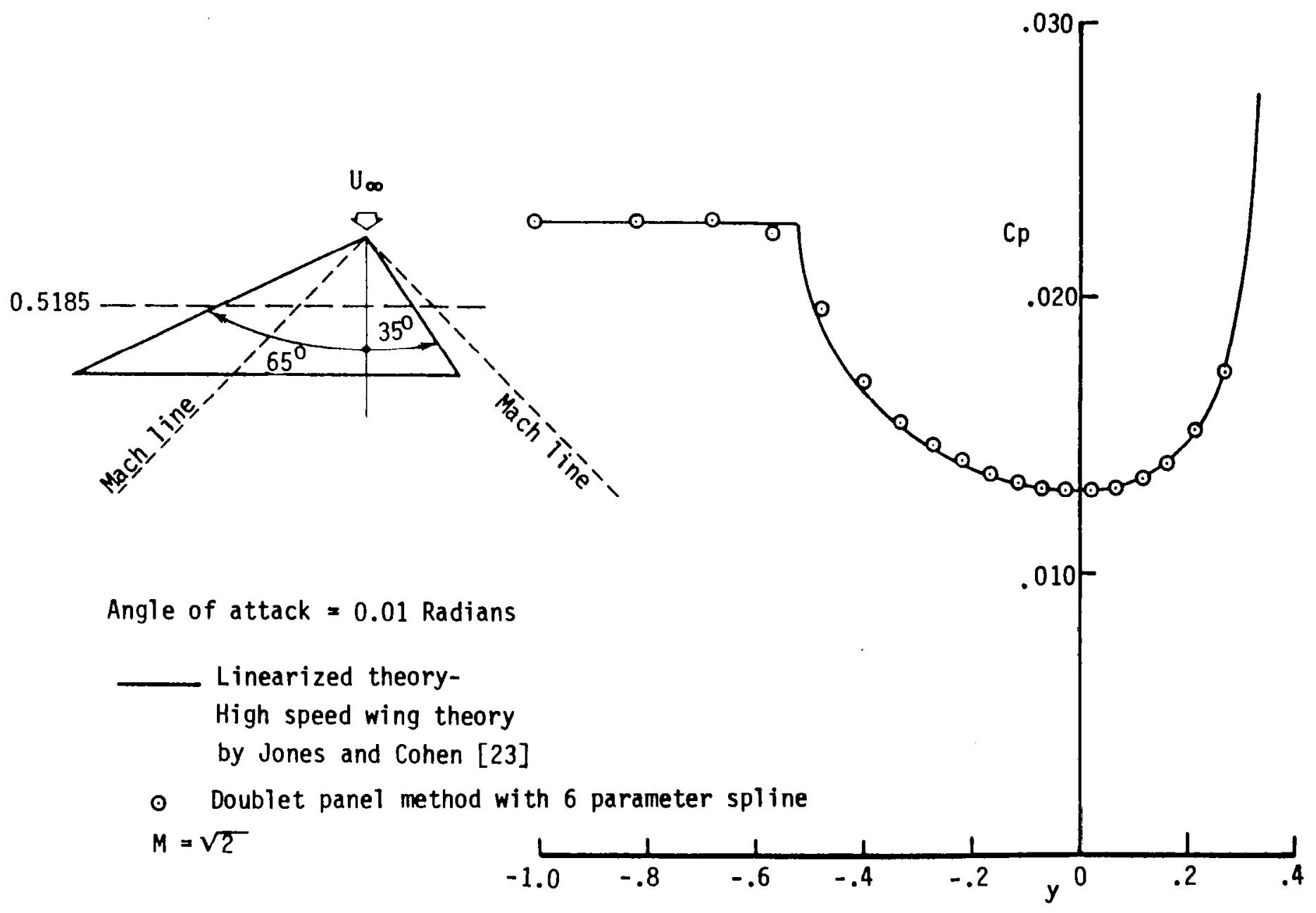

Figure 38.-Comparison of Pressure Distribution From Planar Doublet Panel Method With Linearized Theory For a Yawed Delta Wing 


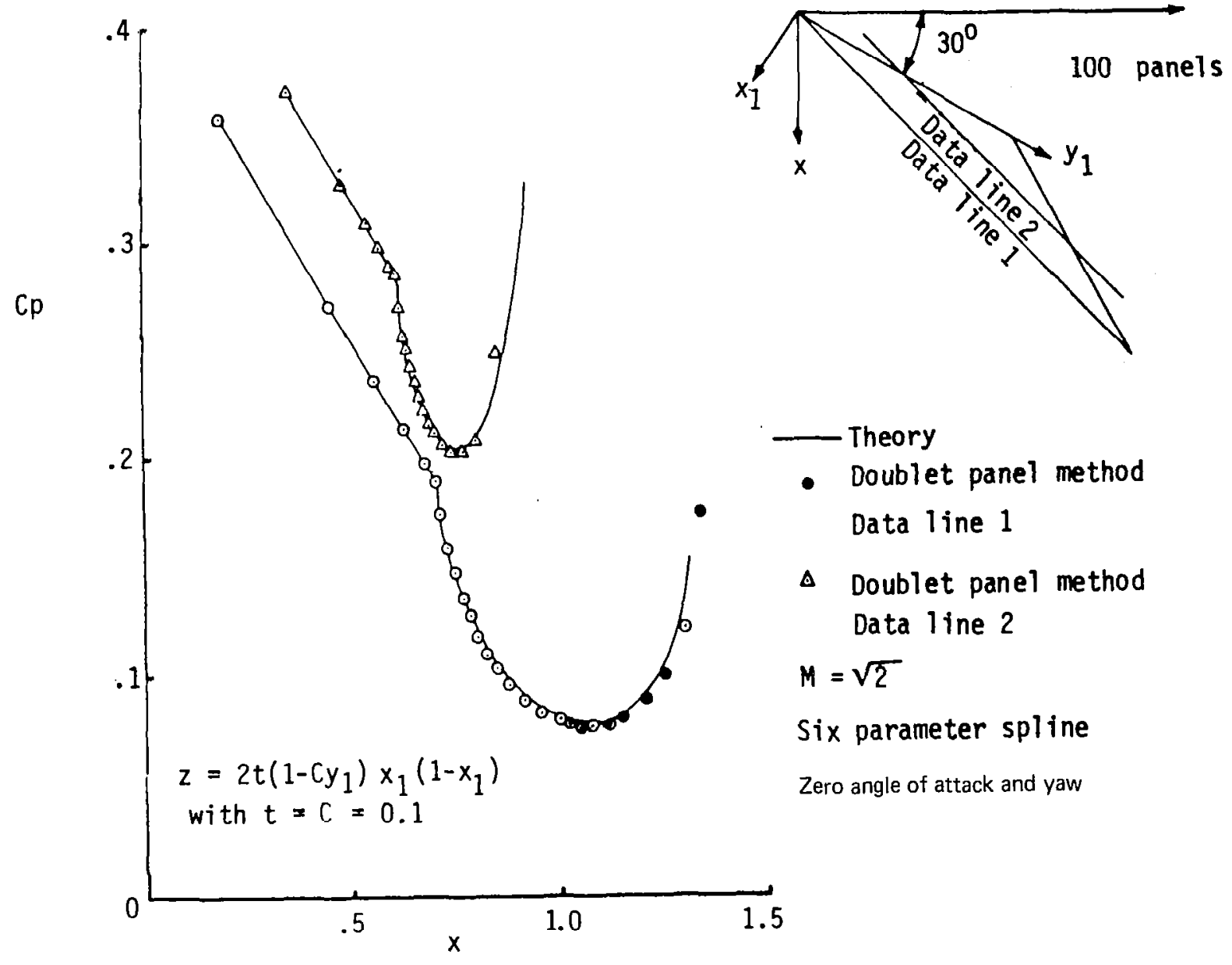

Figure 39.-Comparison of Pressure Distribution on Parabolic Cambered Wing From Planar Doublet Panel Method With Exact Linearized Theory 


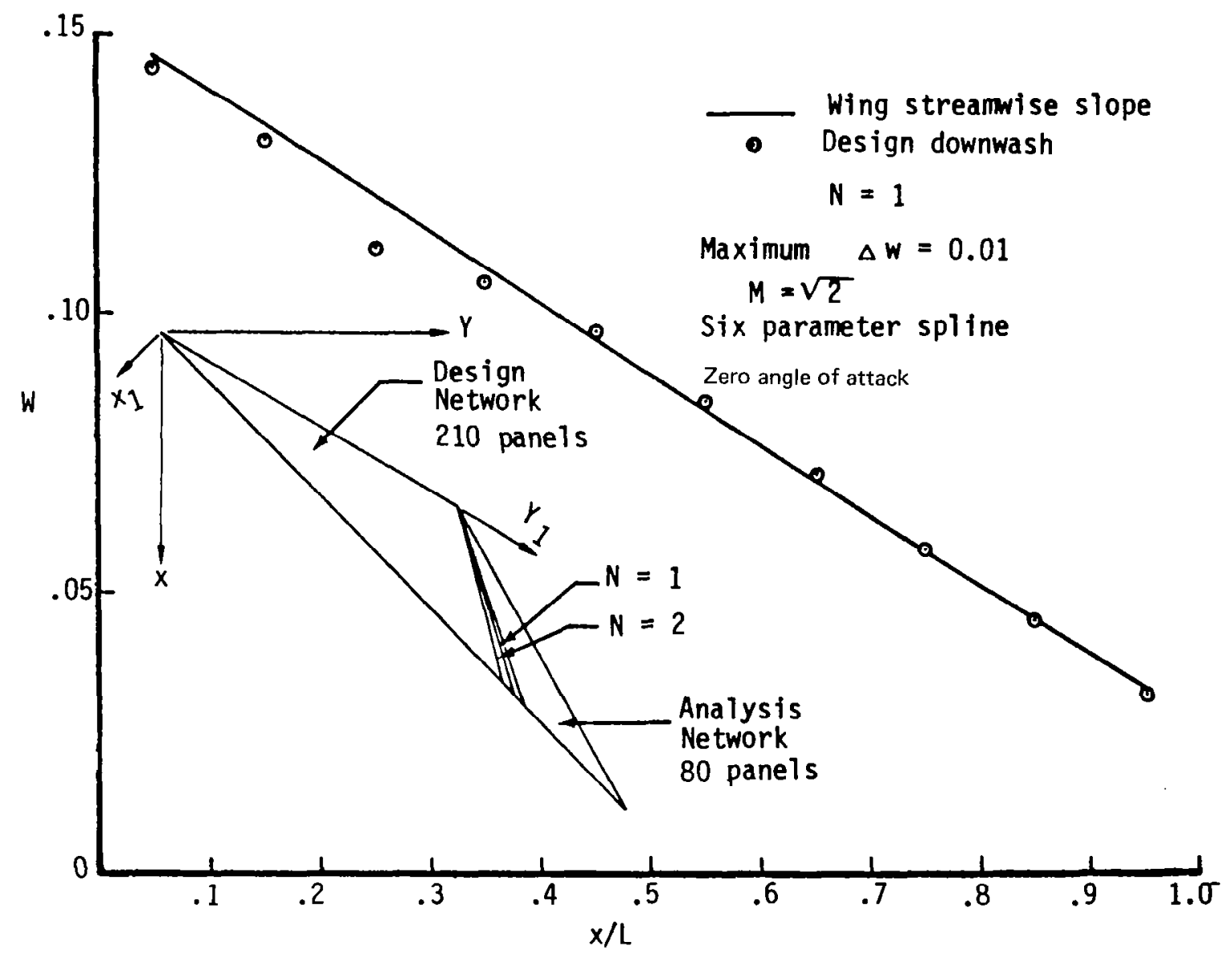

Figure 40.- Comparison of Downwash Computed From Planar Doublet Design Method With The Actual Wing Slopes on The Line N=1 For Cambered Wing in Figure 39 


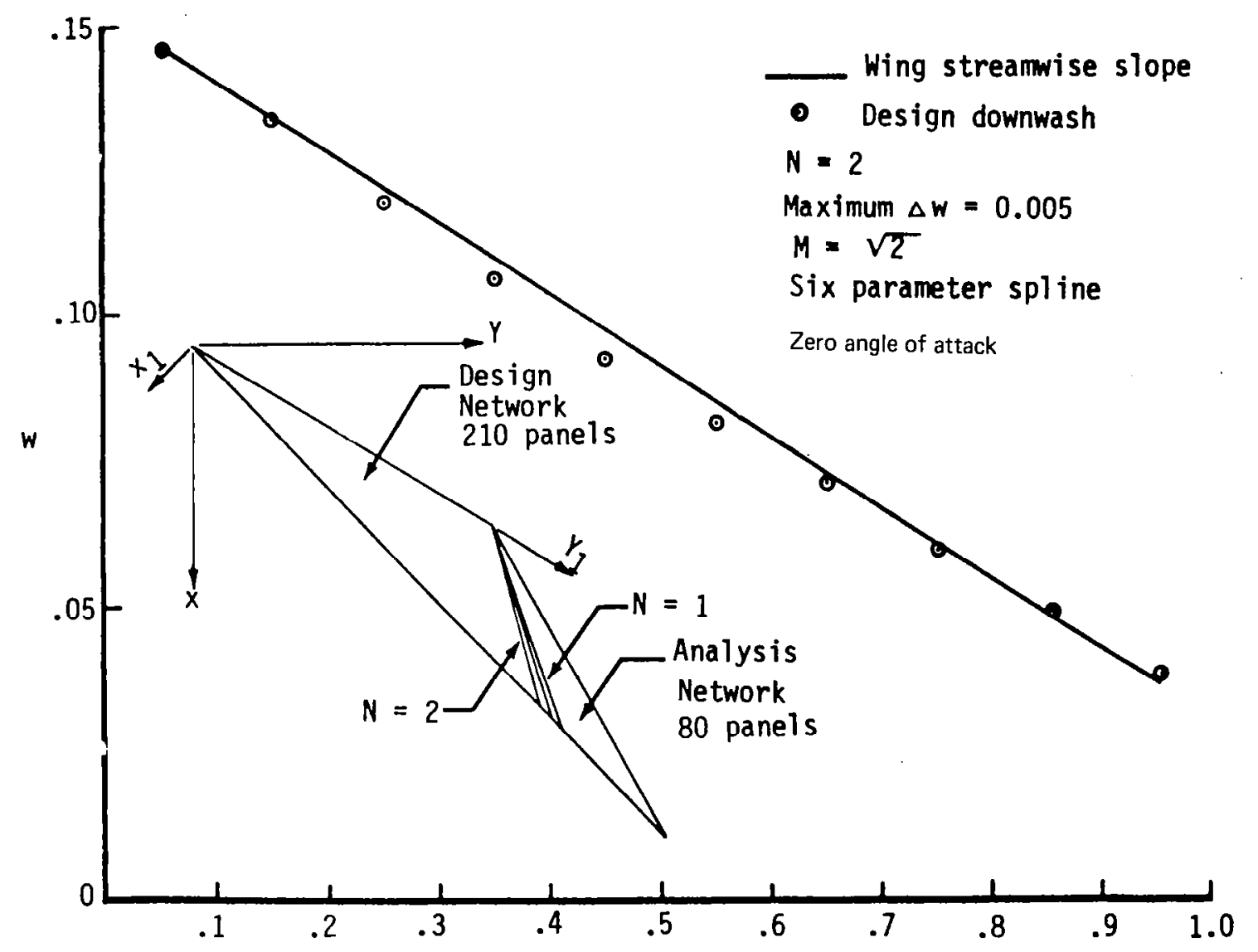

Figure 41.-Comparison of Downwash Computed From Planar Doublet Design Method With Actual Wing Slopes on The Line $N=2$ For Cambered Wing in Figure 39 


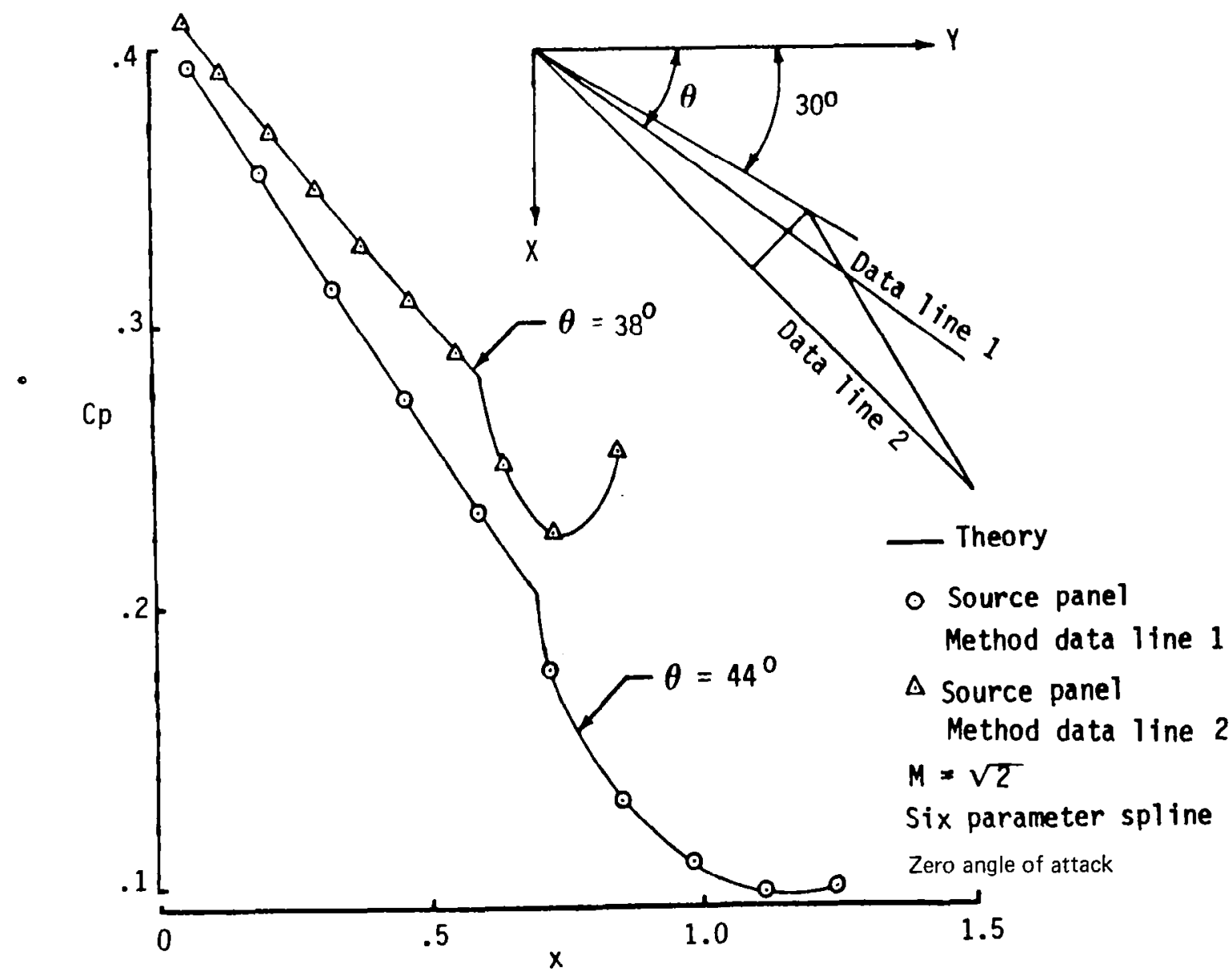

Figure 42.- Comparison of Pressure Distribution on Symmetric Thick Wing Having Same Upper Surface Slopes as in Figure 39 With The Exäct Linearized Theory Solution 


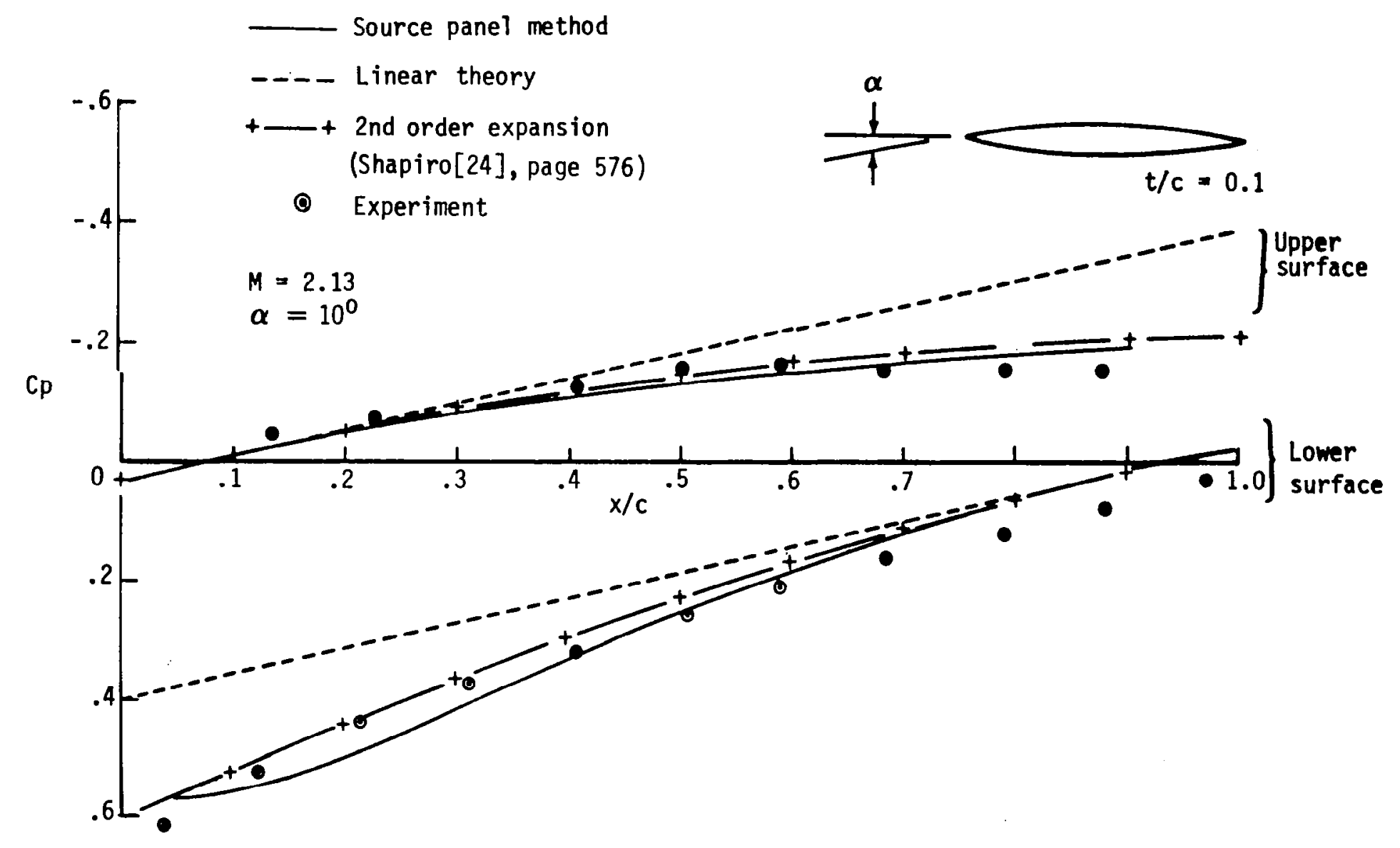

Figure 43.-IIlustrating Advantage of Surface Paneling With Exact Boundary Conditions Over Planar Paneling With Linearized Boundary Conditions 

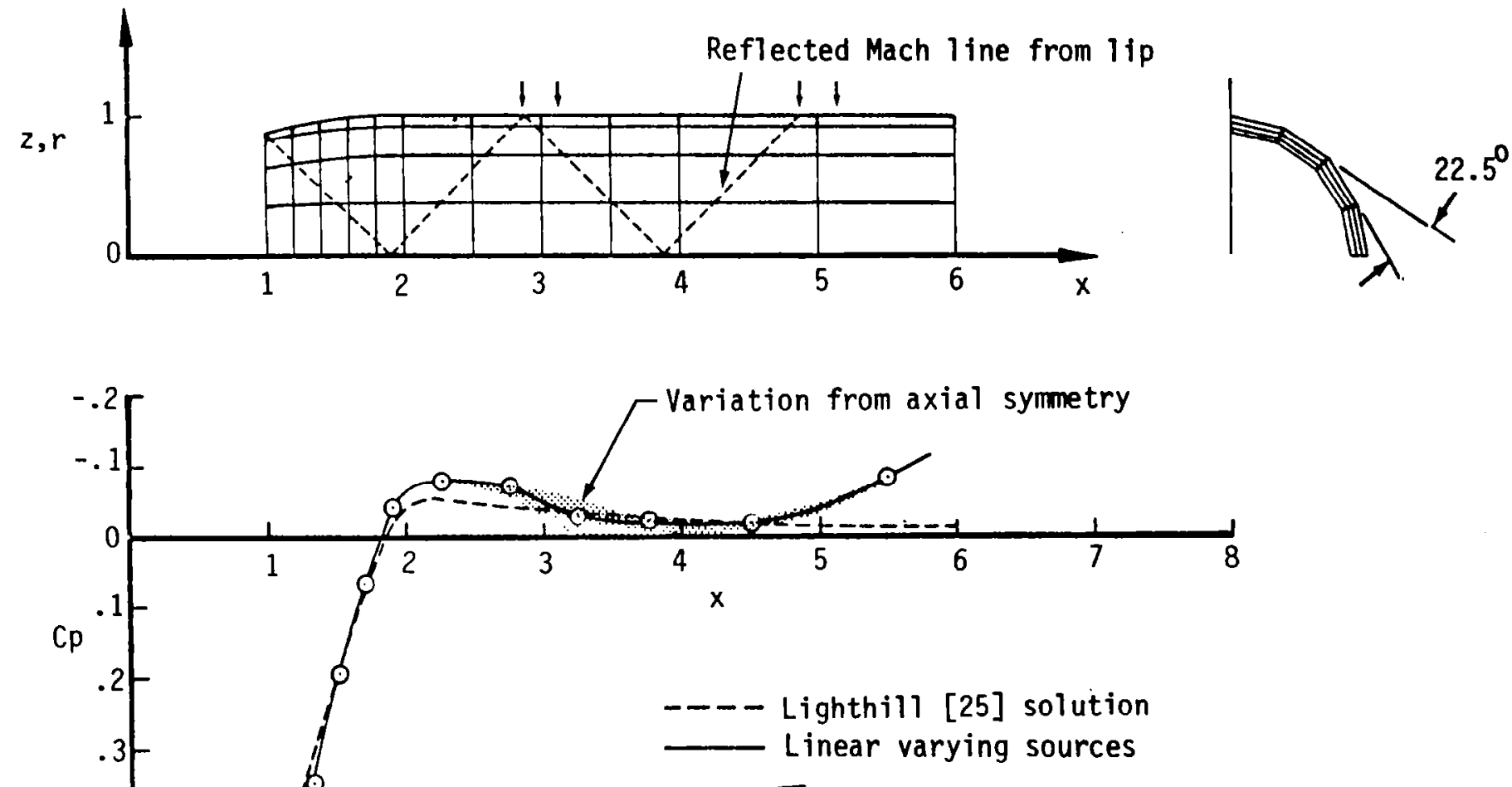

$M=\sqrt{2}$ 

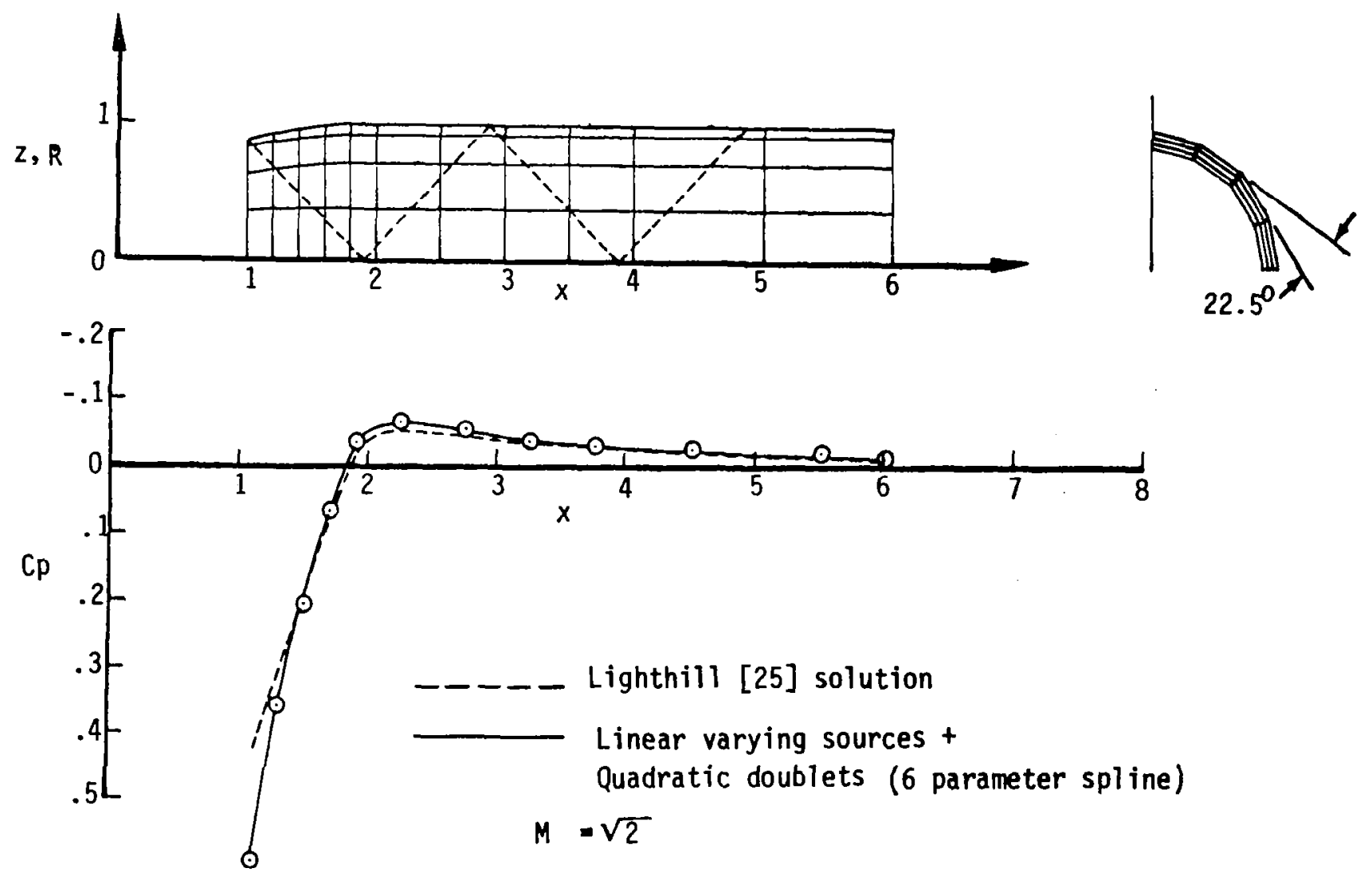

Figure 45.-Coefficient of Pressure on Outer Surface of Inlet Nacelle by Combined DoubietSource Panel Method 


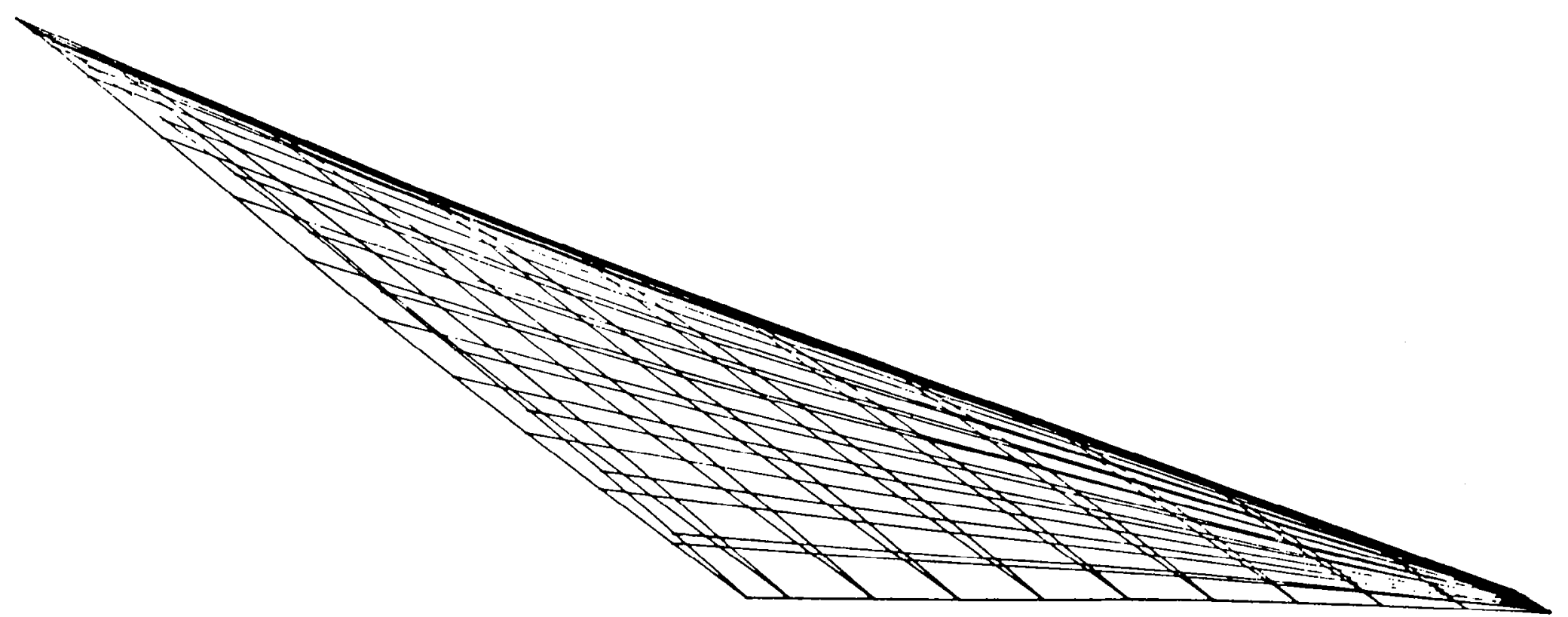

Figure 46. - Paneling on Car/son Wing $2 T$ 


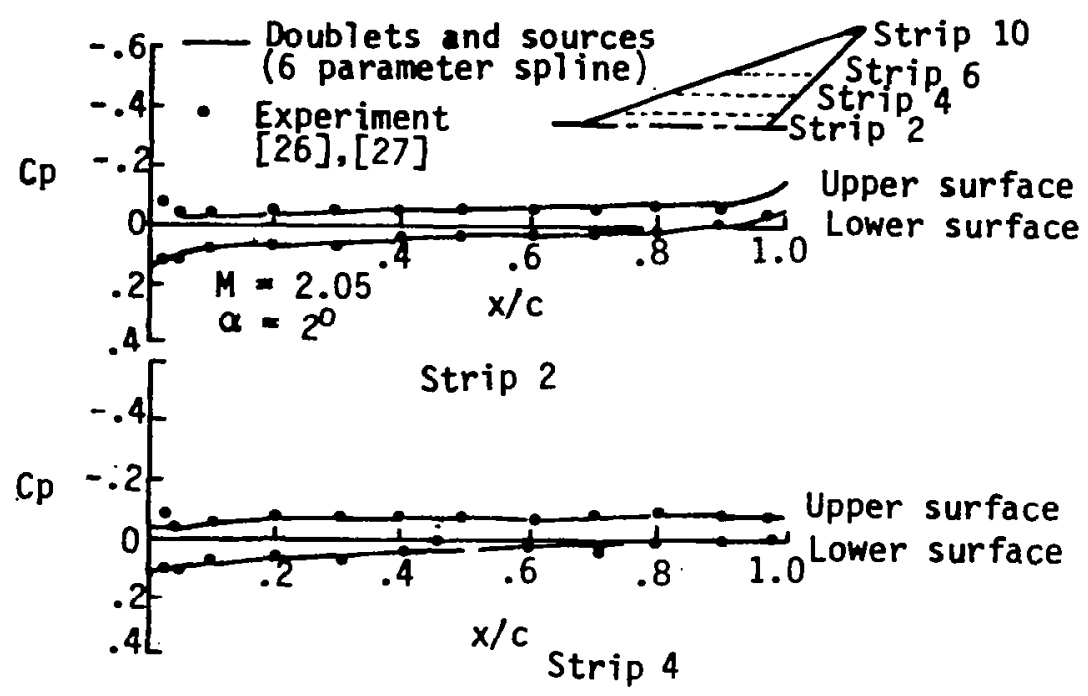

Figure 47.-Comparison of Pressure Distribution Computed From Combined Doublet-source Panel Method With Experiment on Strips 2 and 4 of Carlson Wing $2 T$

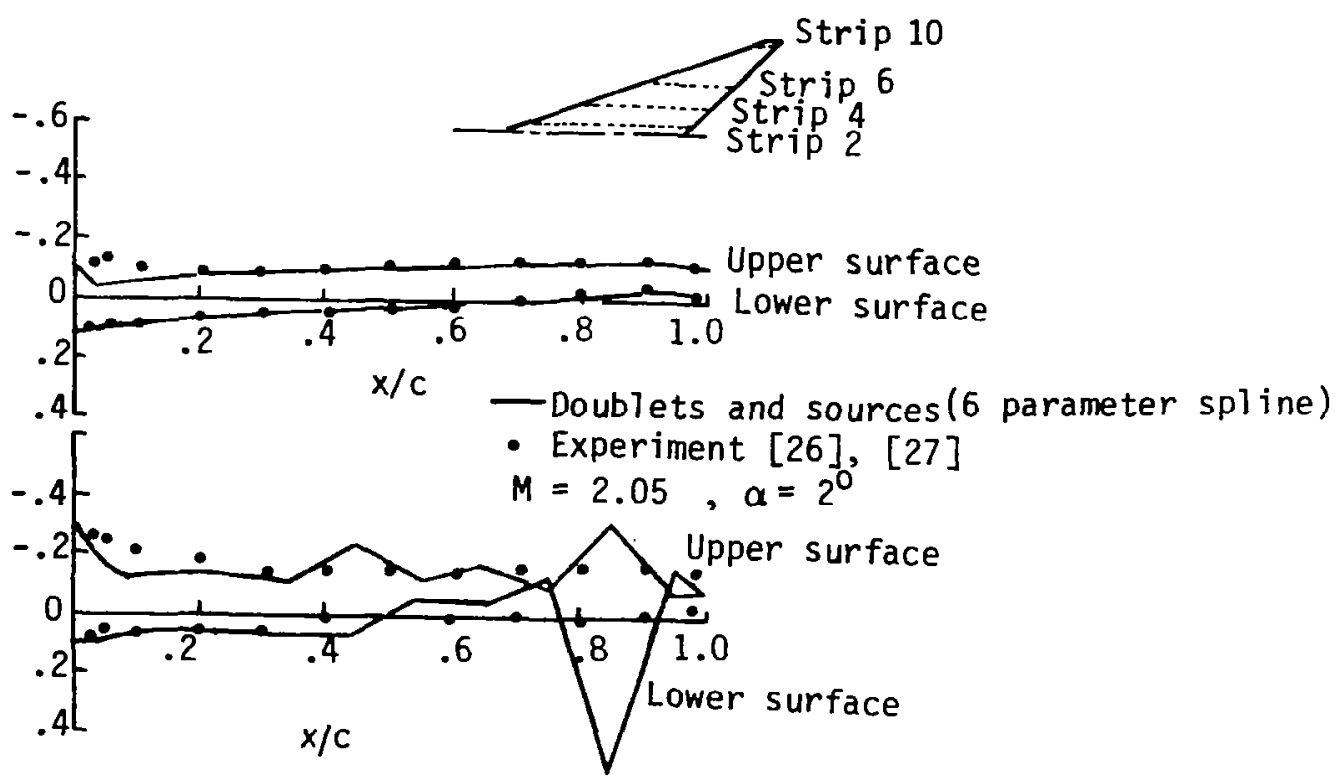

Figure 48. - Comparison of Pressure Distribution Computed From Combined Doublet-Source Panel Method With Experiment on Strips 6 and 10 of Carlson Wing $2 T$ 


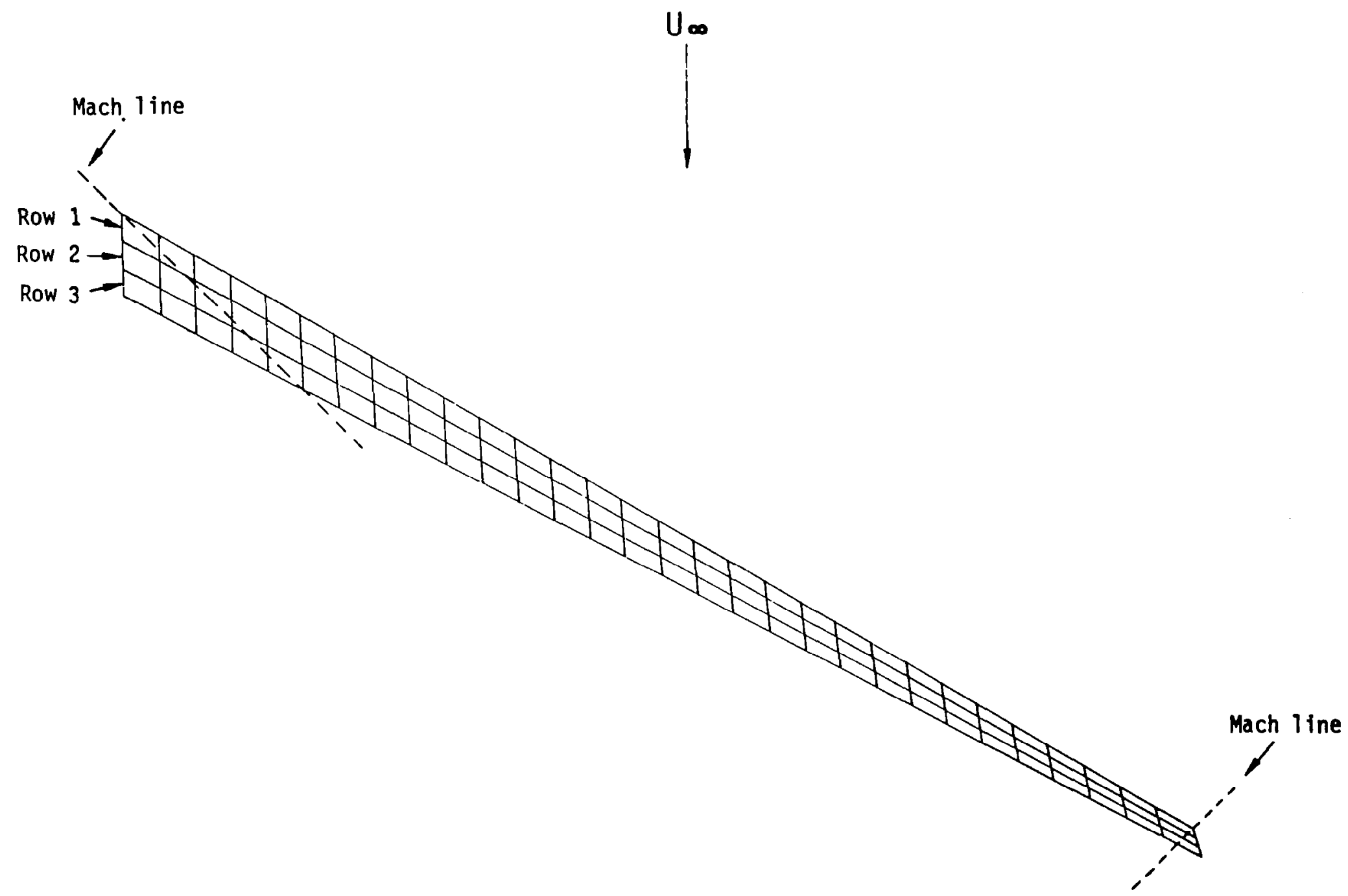

Figure 49.-Paneling of Leading Edge Region of The Parabolic Arc Cambered Wing in Figure 39 Which Showed Instability in The Solution 


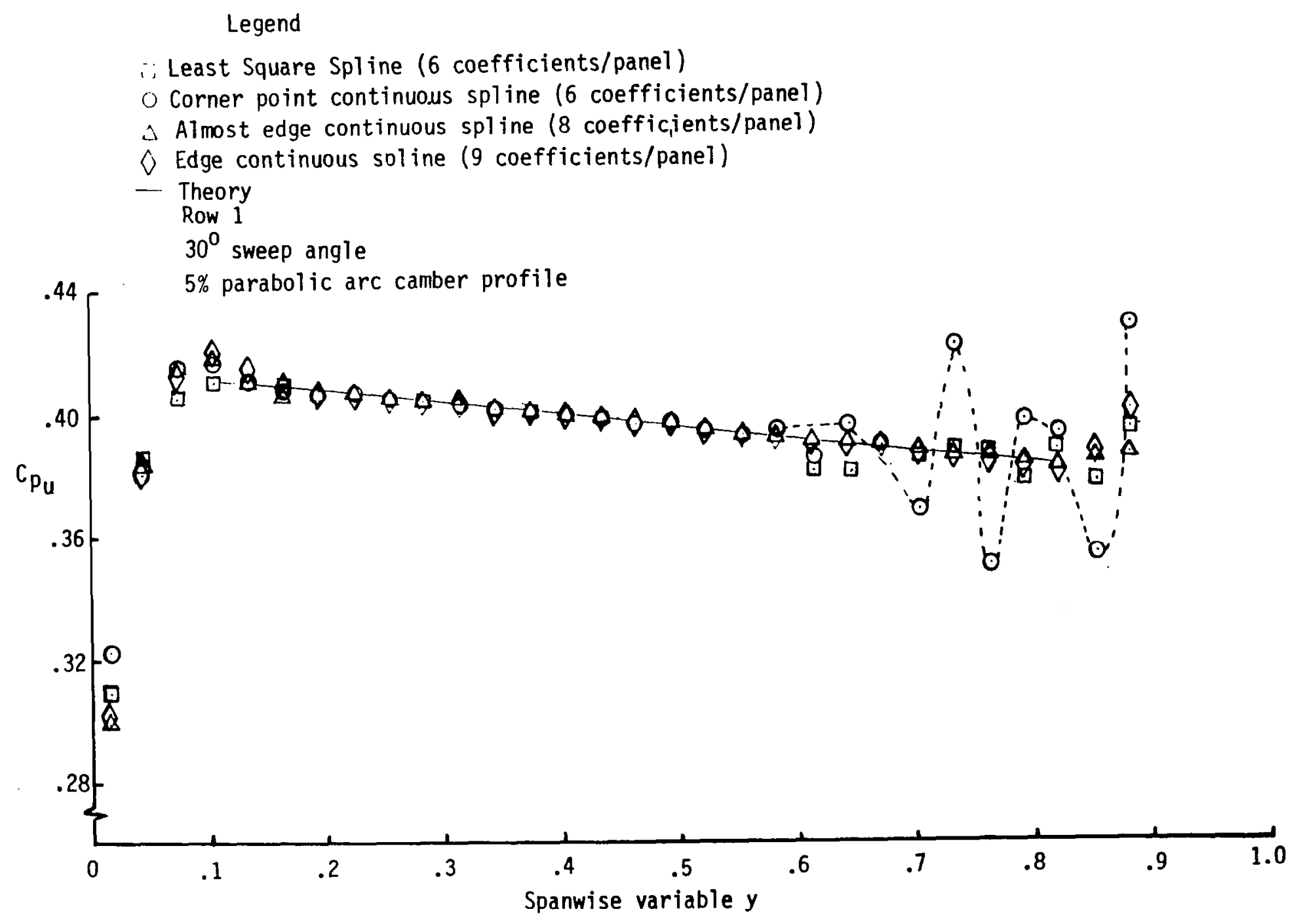


Least square spline (6 coefficients/pane1)

O Corner point continuous spline ( 6 coefficients/panel)

$\triangle$ Almost edge continuous spline ( 8 coefficients/ panel)

$\diamond$ Edge continuous spline (9 coefficients/panel)

- Theory Row 2

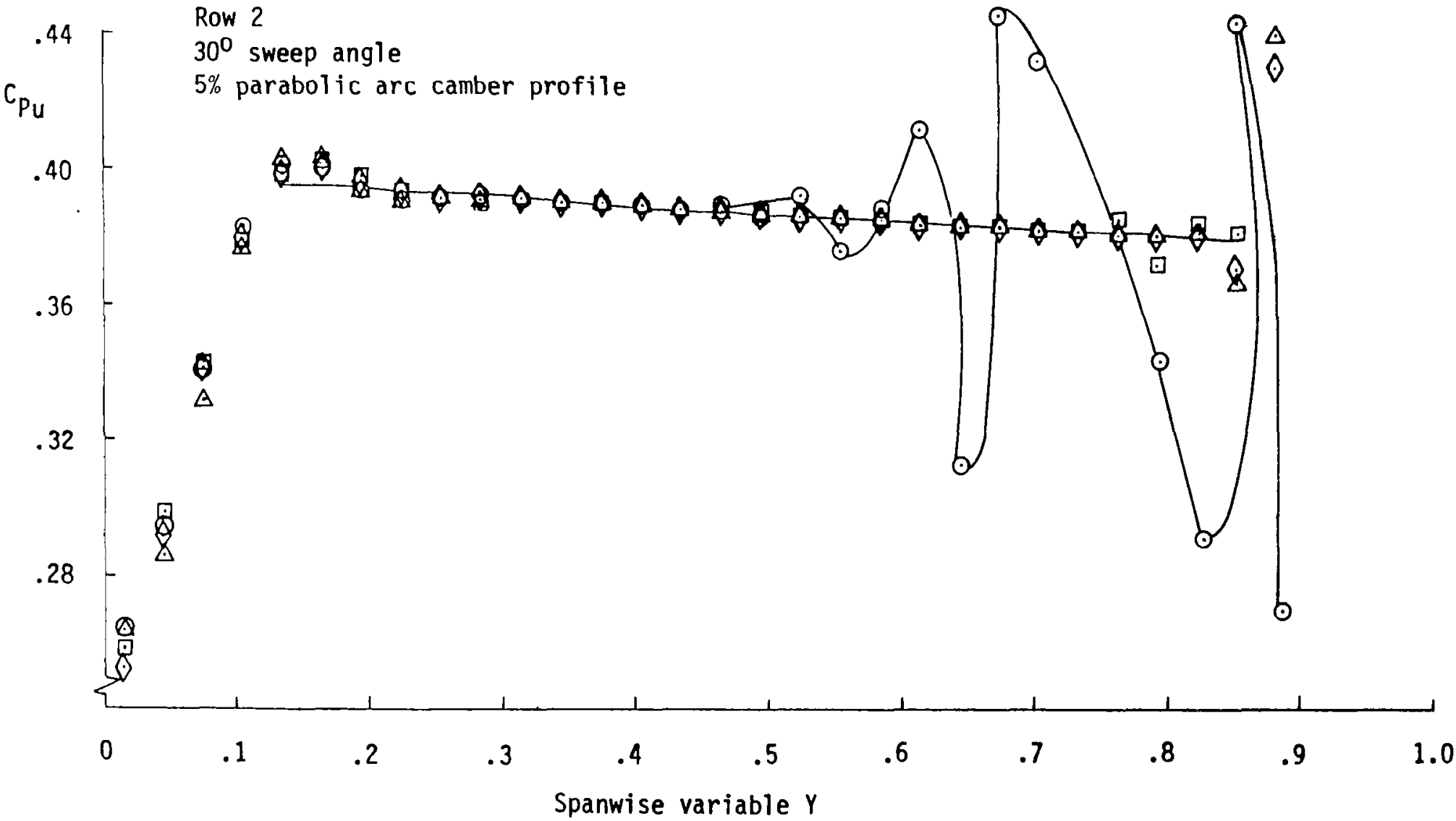

Figure 51.-Comparison of Pressure Distribution on Row 2 of Upper Surface of The Parabolic Cambered Wing Using Three Different Splines And The Paneling of Figure 49 
Legend

$\square$ Least square spline (6 coefficients/panel)

$.44-O$ Corner point continuous spline ( 6 coefficients/panel)

$\triangle$ Almost edge continuous spline (8 coefficients/panel)

$\checkmark$ Edge continuous spline (9 coefficients/panel)

- Theory

$.40-30^{\circ}$ sweep angle

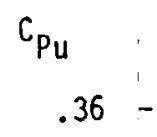

$5 \%$ parabolic arc camber profile

$\triangle \stackrel{\Delta}{8} \mathscr{Q}$

.

$\odot$

$.32:$
0
$.28 \div \quad 8$

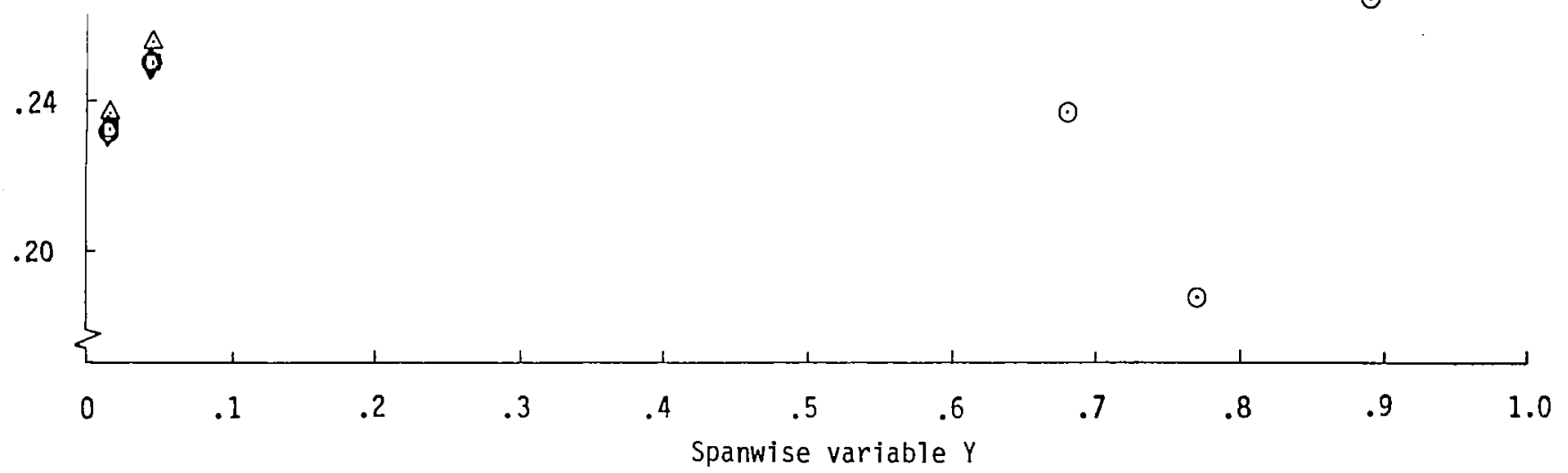

Figure 52.-Comparison of Pressure Distribution on Row 3 of Upper Surface of The Parabolic Cambered Wing Using Three Different Splines And The Paneling in Figure 49 

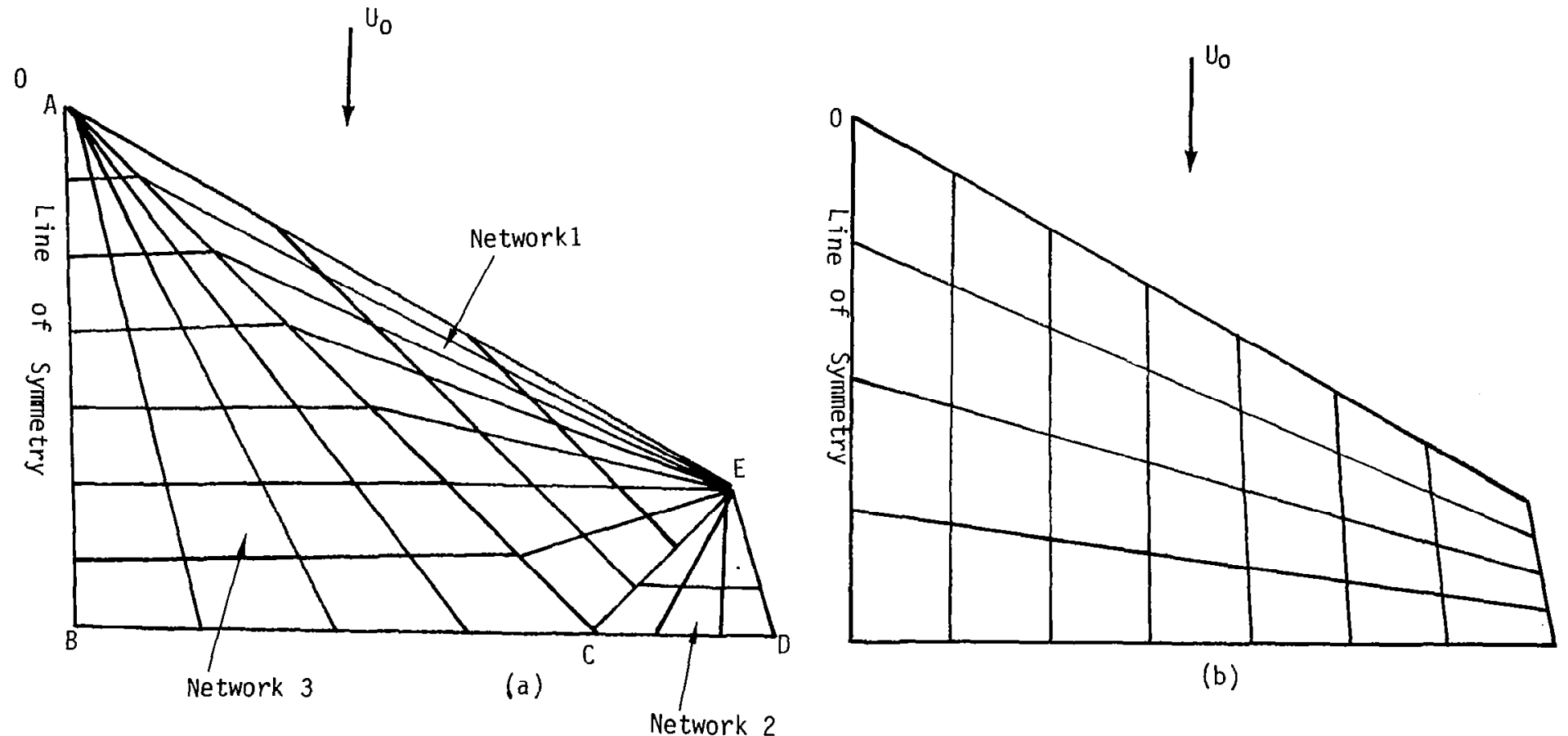

(b)

Figure 53.-Examples of Successful And Unsuccessful Paneling of The Parabolic Cambered Wing 

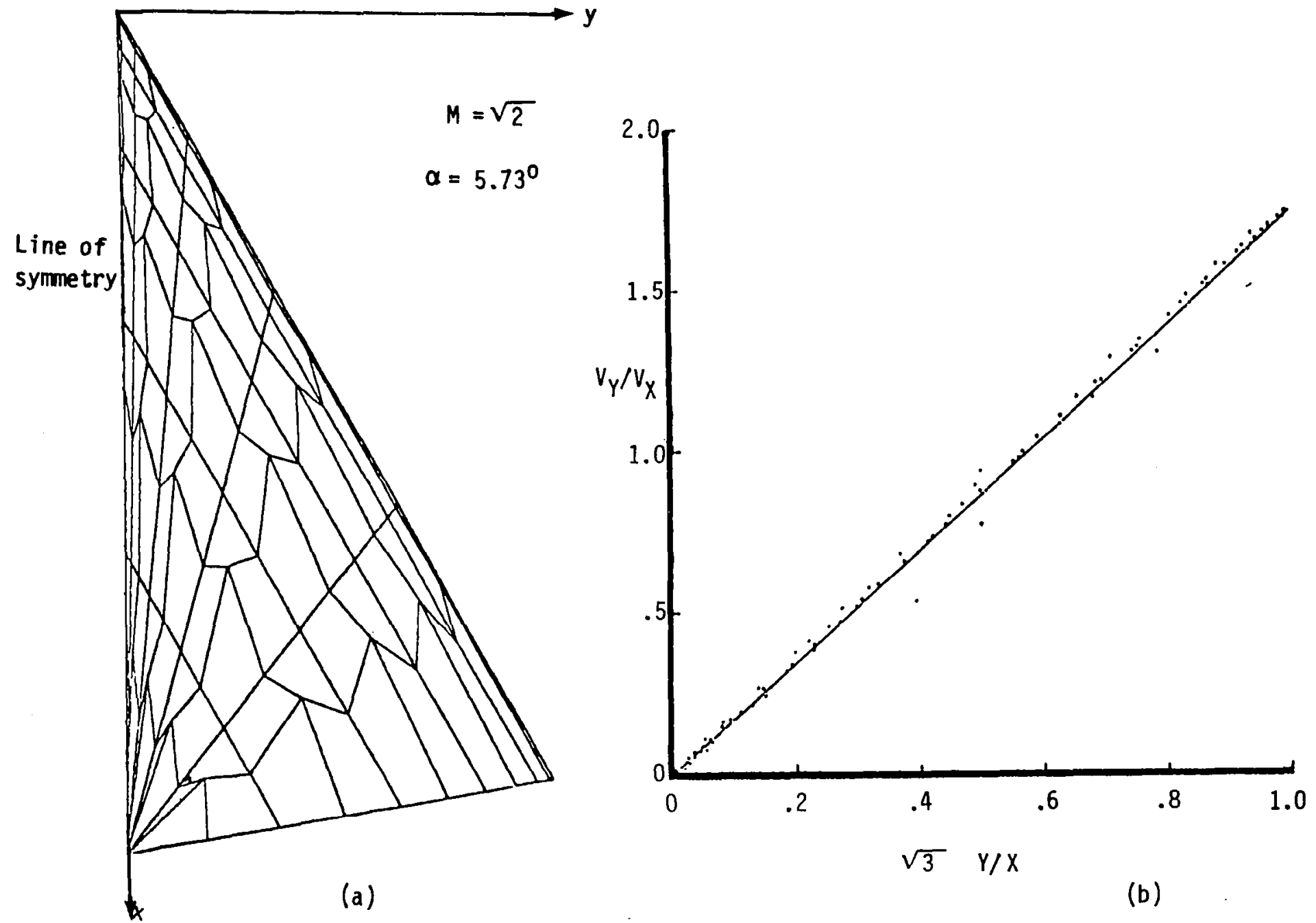

Figure 54.-Arbitrary Paneling of The Flat Delta Wing Used to Test The Stability of The Nine Parameter Doublet Spline 

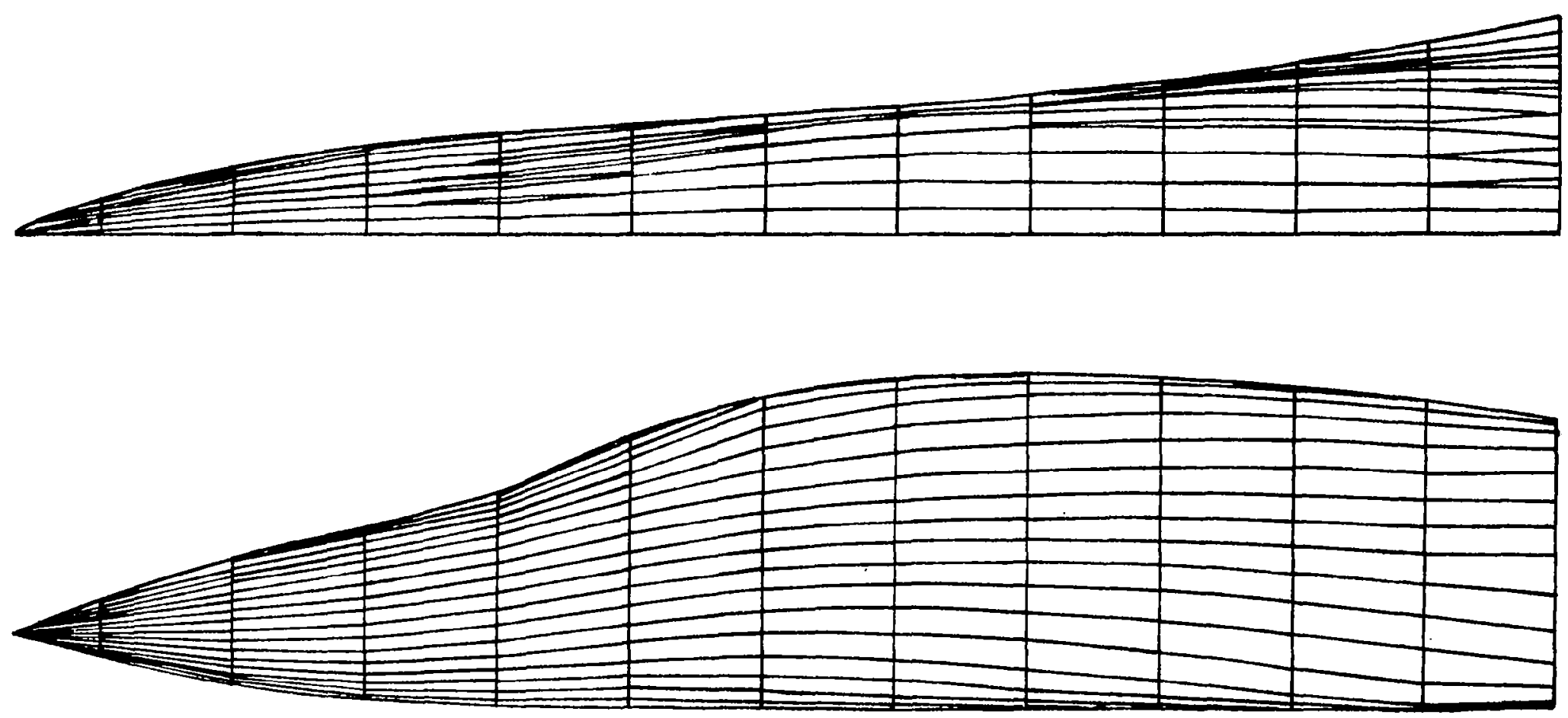

Figure 55.-Paneling on The Forebody of The B-1 Bomber 


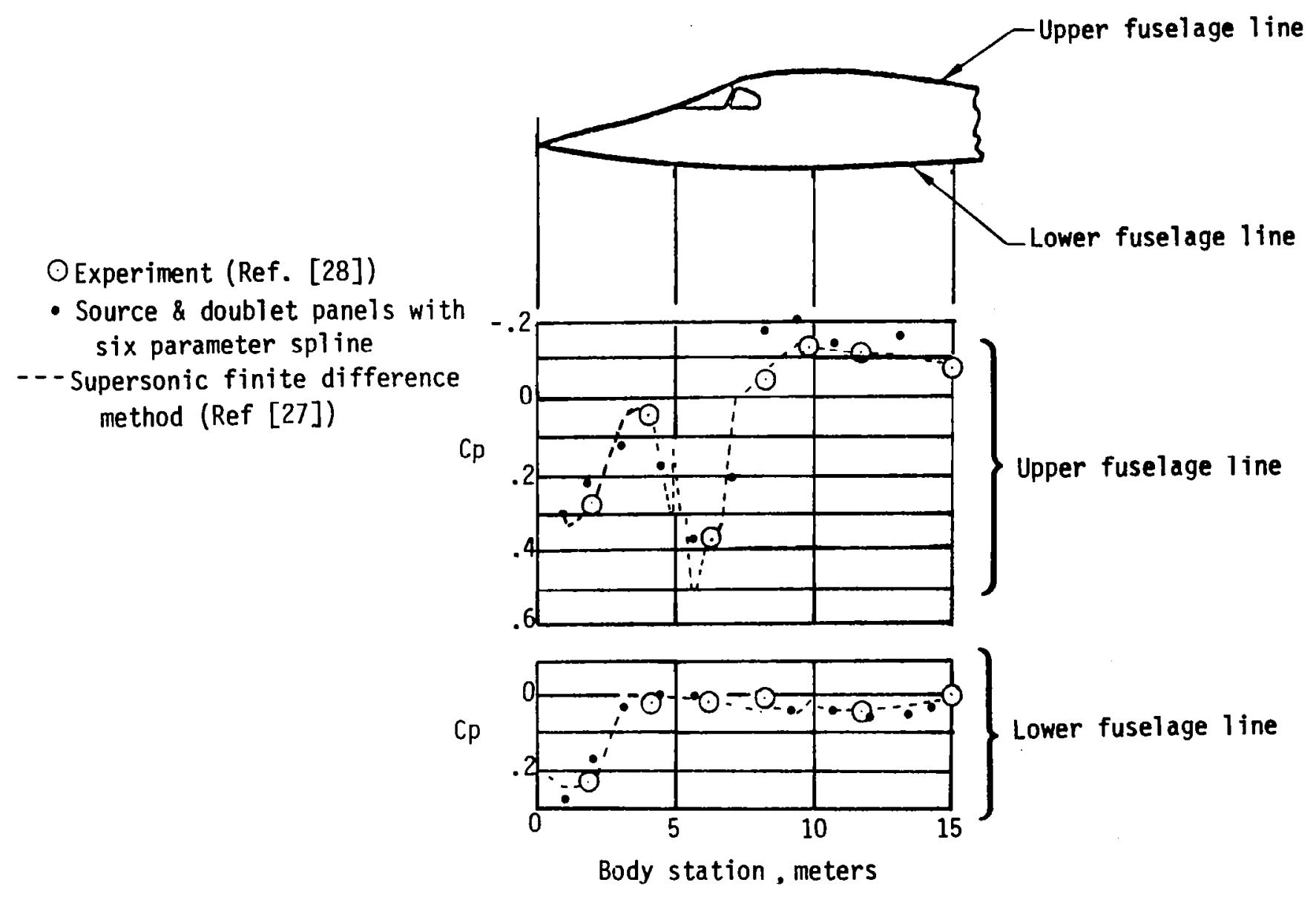
Doublet Gradient With Experiment And With The Finite Difference Solution 
- Experiment (Ref. [28])

- Source \& doublet panels with six parameter spline

-.---Supersonic finite difference method

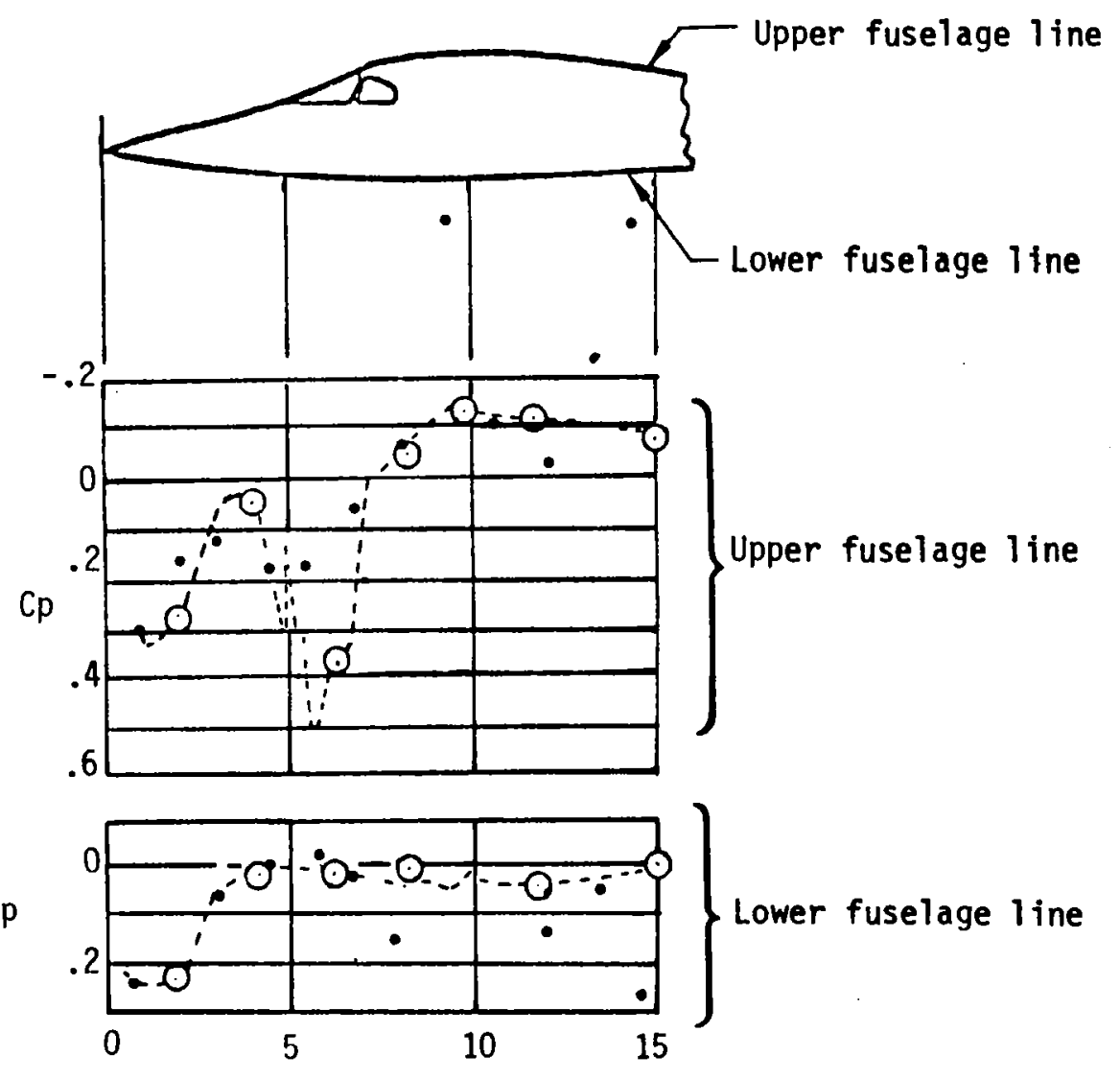

Body station, meters

Figure 57.-Comparison of The Pressure Coefficient on The B-1 Forebody Computed From The Aerodynamic Influence Coefficients Using The Six Parameter Spline With Experiment And With The Finite Difference Solution 
○ Experiment [Ref. 28]

- Source \& doublet panels with nine parameter spline -..--Supersonic finite difference method

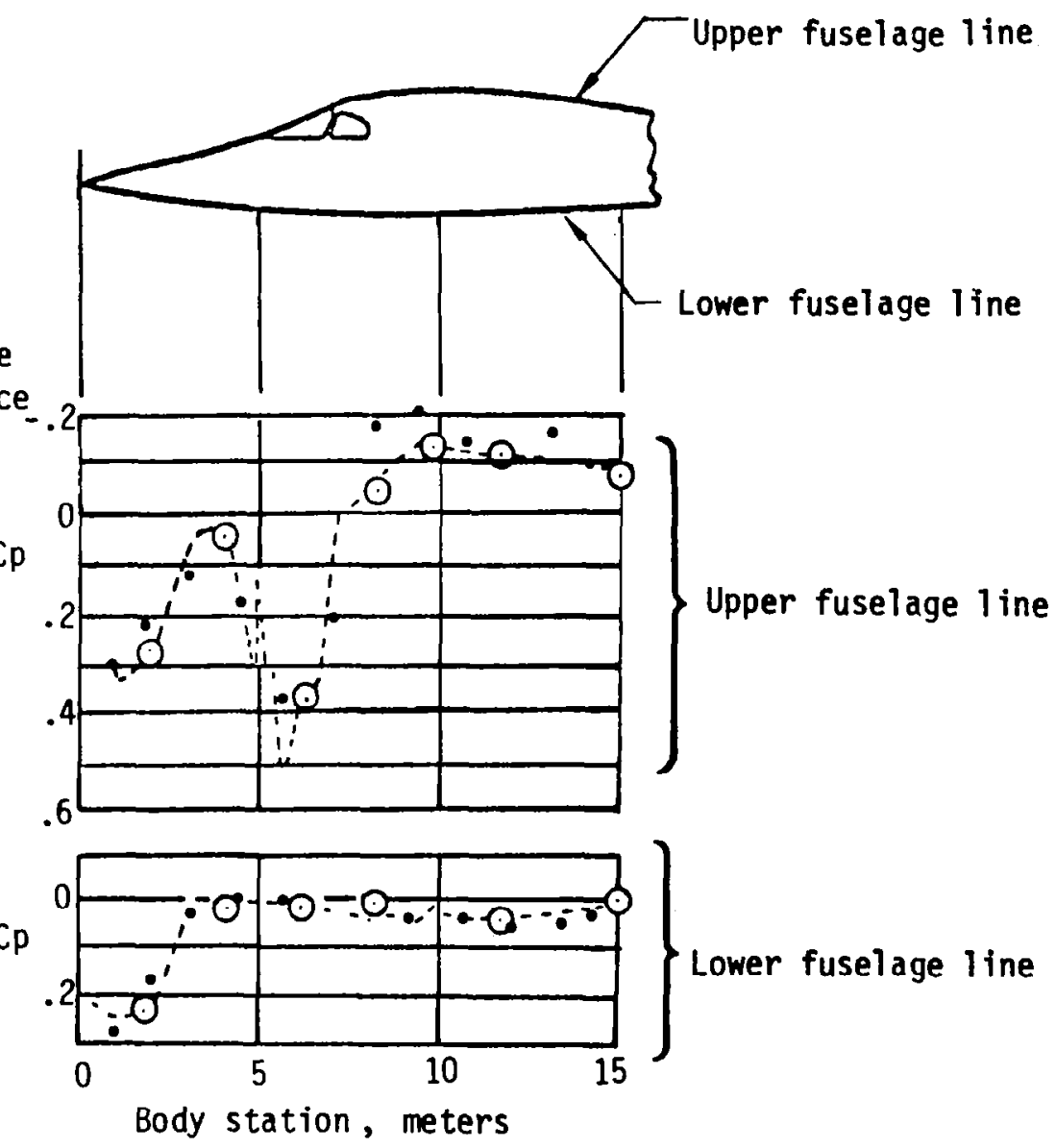

Figure 58.-Comparison of The Pressure Coefficient on The B-1 Forebody Computed From The Aerodynamic Influence Coefficients Using The Nine Parameter Spline With Experiment And With The Finite Difference Solution 


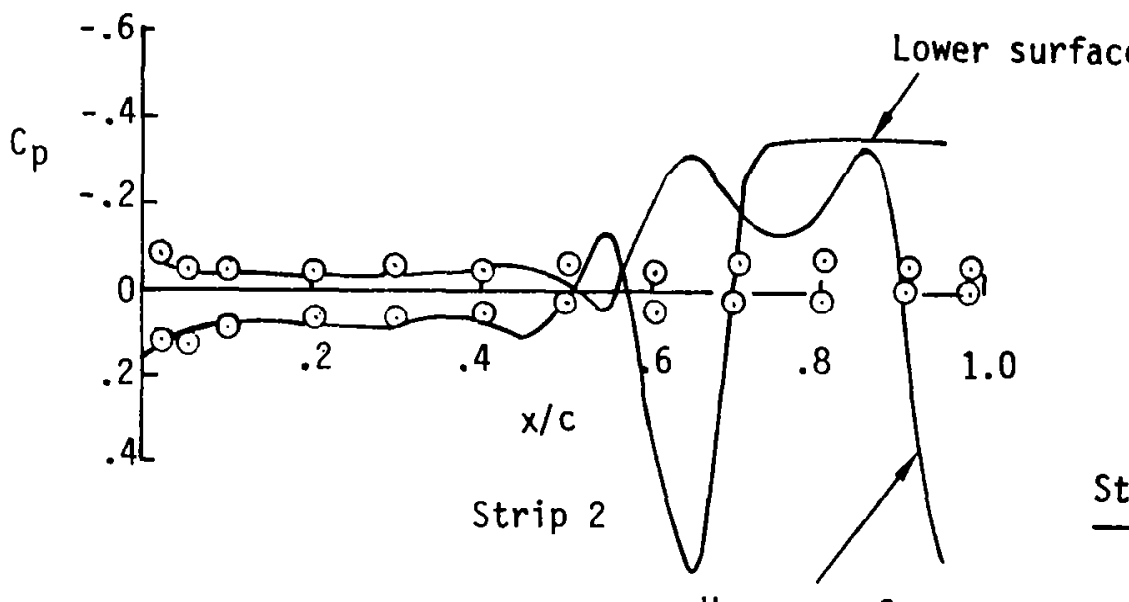

$$
\begin{aligned}
& M=2.05 \\
& a=2^{0}
\end{aligned}
$$
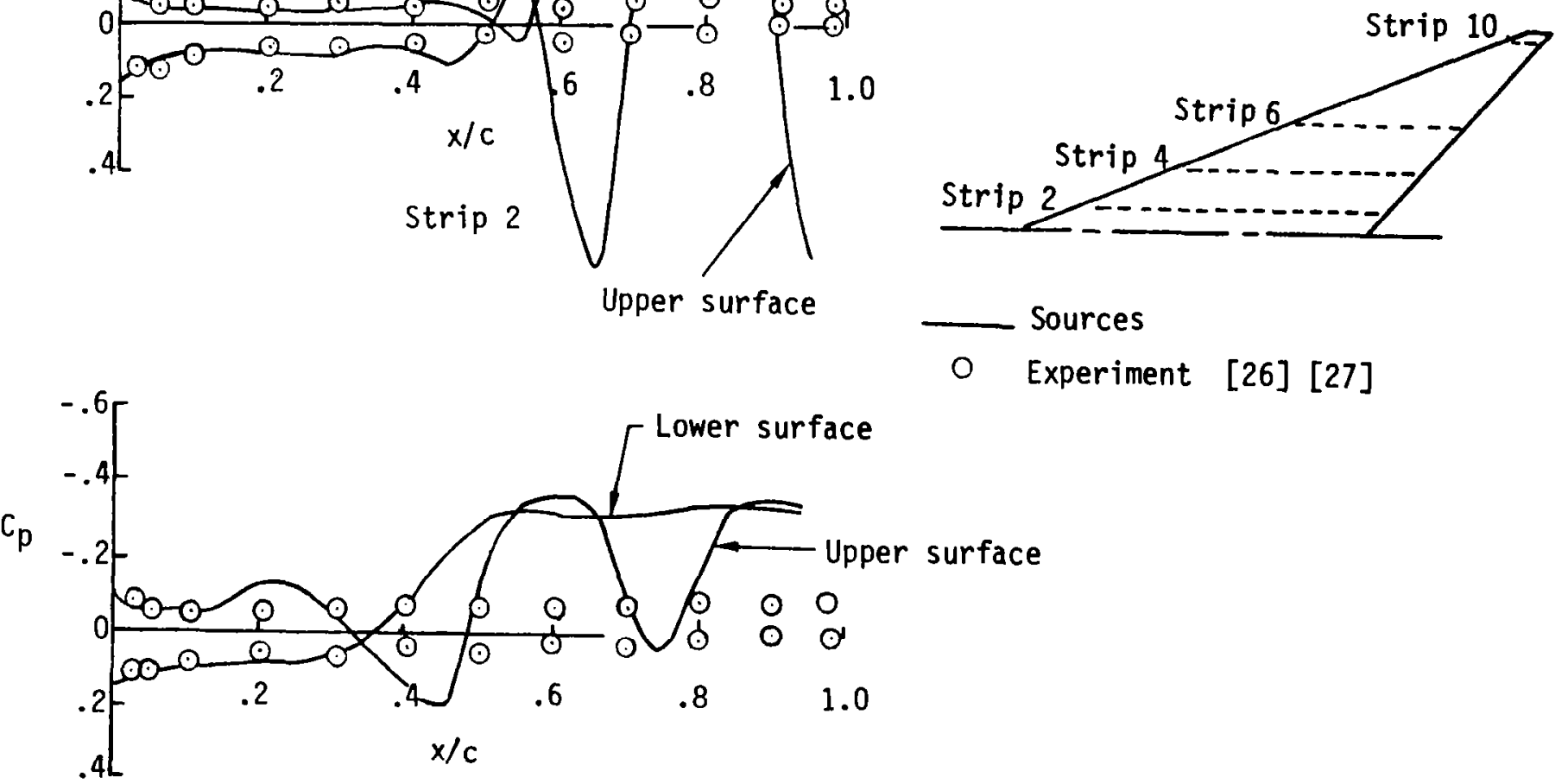

\section{Sources}

Experiment [26] [27]

\section{Strip 4}

Figure 59. - Comparison of Pressure Coefficient on The Carlson Wing Computed by Sources Alone With Experimental Measurements at Span Stations 2 and 4 


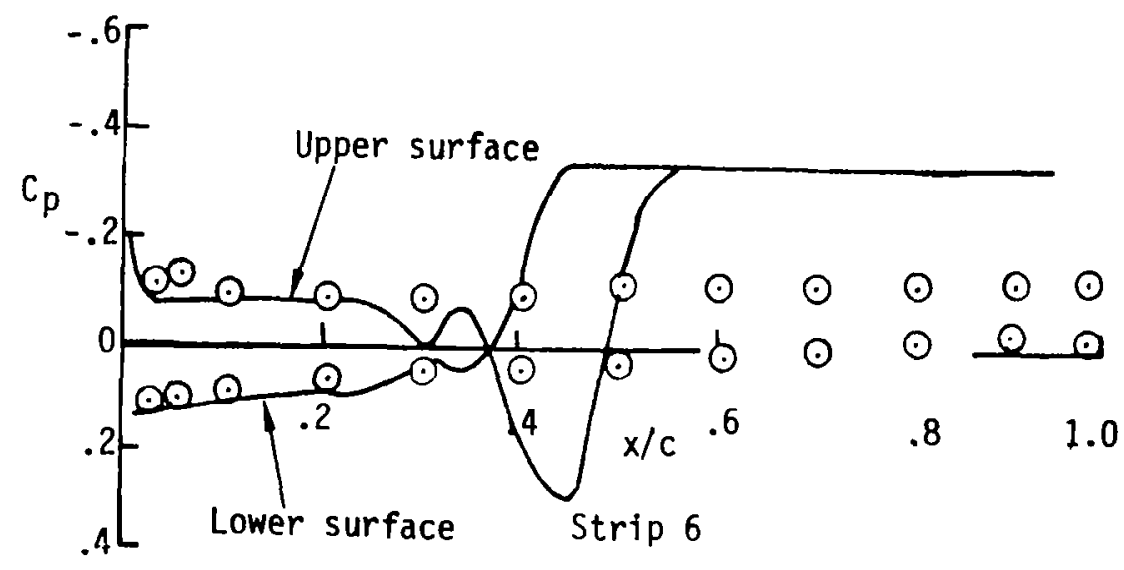

$$
\begin{aligned}
& M=2.05 \\
& \alpha=2^{0}
\end{aligned}
$$
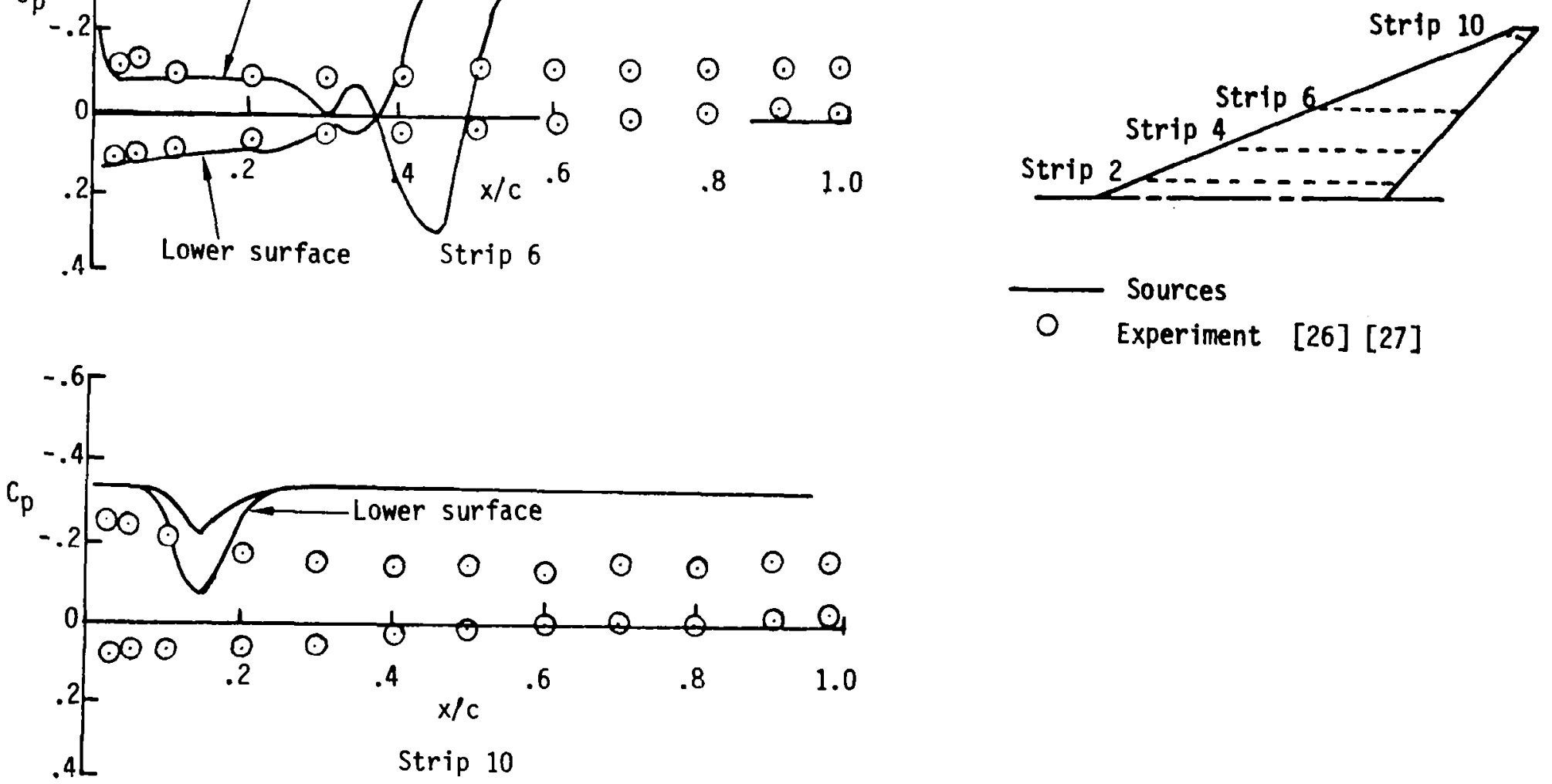

Figure 60.-Comparison of Pressure Coefficient on The Carlson Wing Computed by Sources Alone With Experimental Measurements at Span Station 6 and 10 


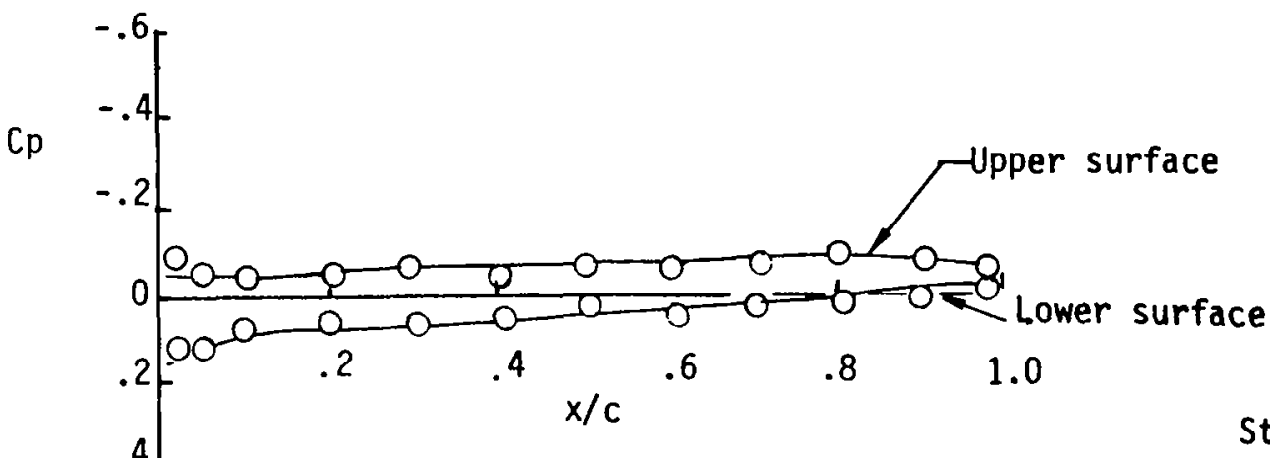

$M=2.05$

$\alpha=2^{0}$

Strip 2

Strip 2

Strip 10

Strip 6

Strip 4

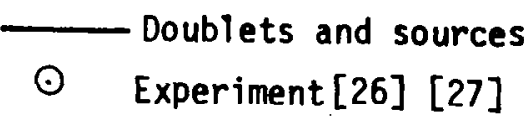

Figure 61.-Comparison of Pressure Coefficient on The Carlson Wing Computed by Combined Doublet-source Pane/s Using The Nine Parameter Spline With Experiment at Span Stations 2 and 4 

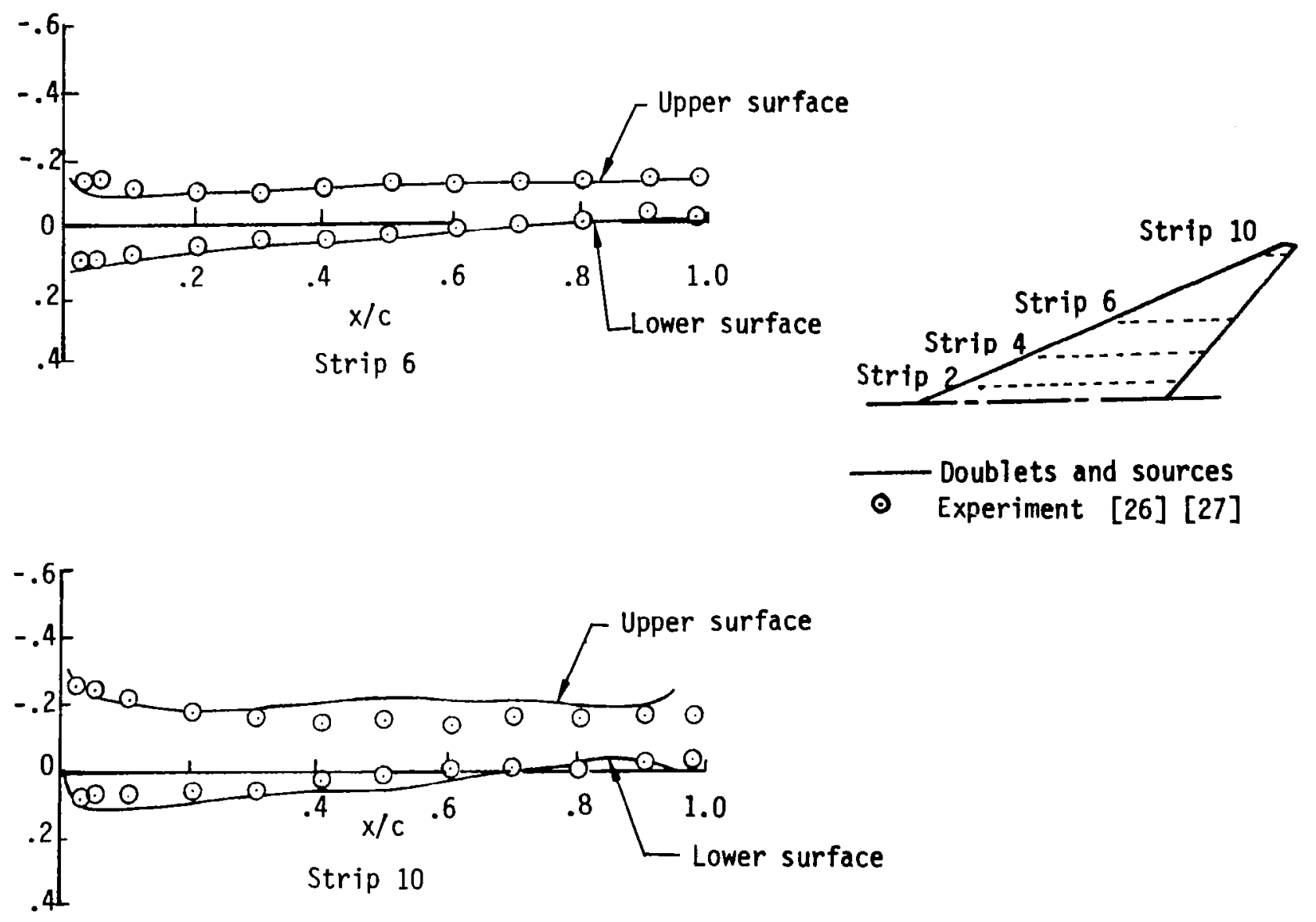

Figure 62.-Comparison of Pressure Coefficient on The Car/son Wing Computed by Combined Doublet-source Pane/s Using The Nine Parameter Spline With Experiment at Span Stations 6 and 10 


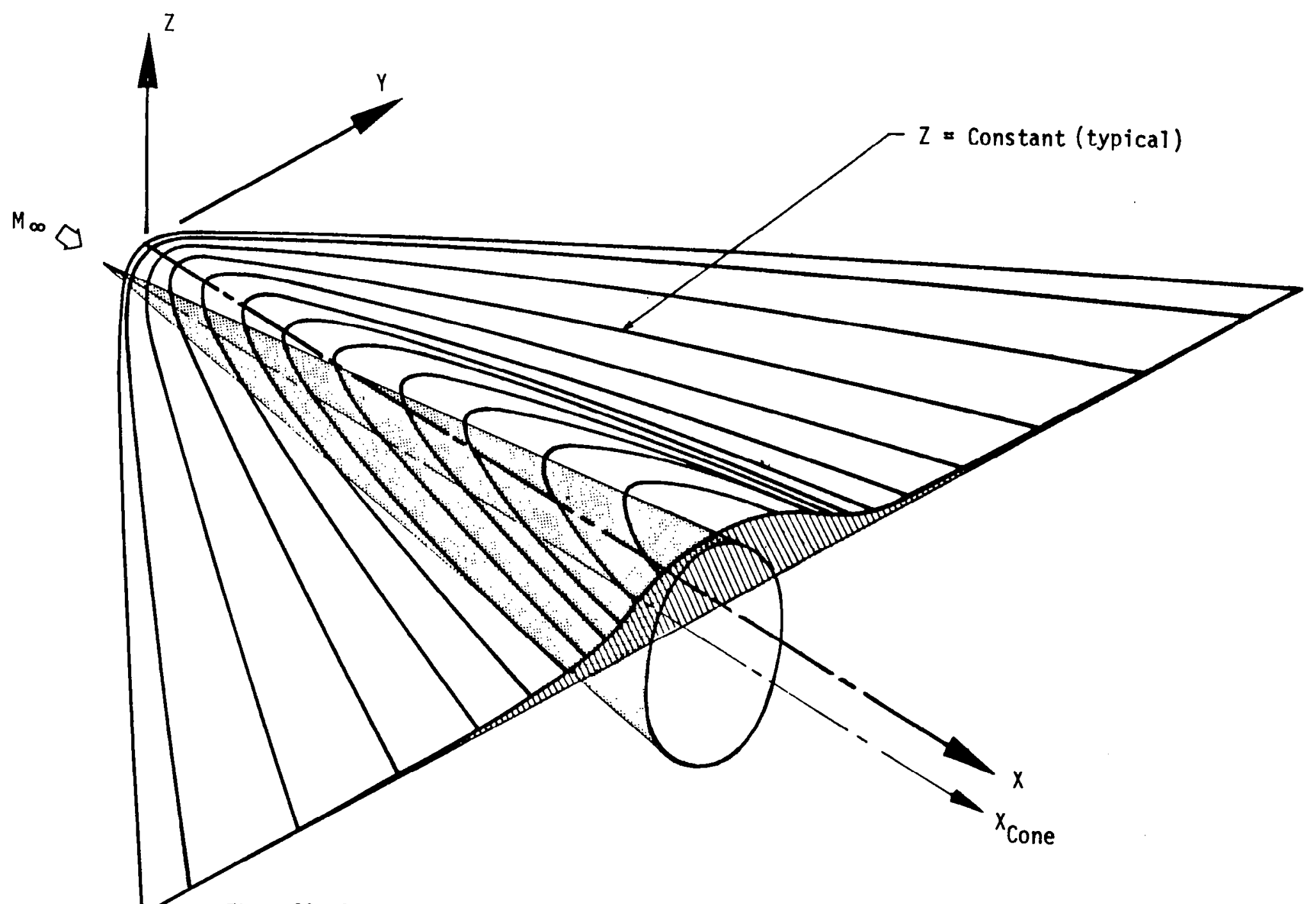

Figure 63.-Stream Surface Taken From Exact Solution For Flow Past Cone And Defined by a Plane Parallel to The Shock Axis Cutting The Conical Shock 

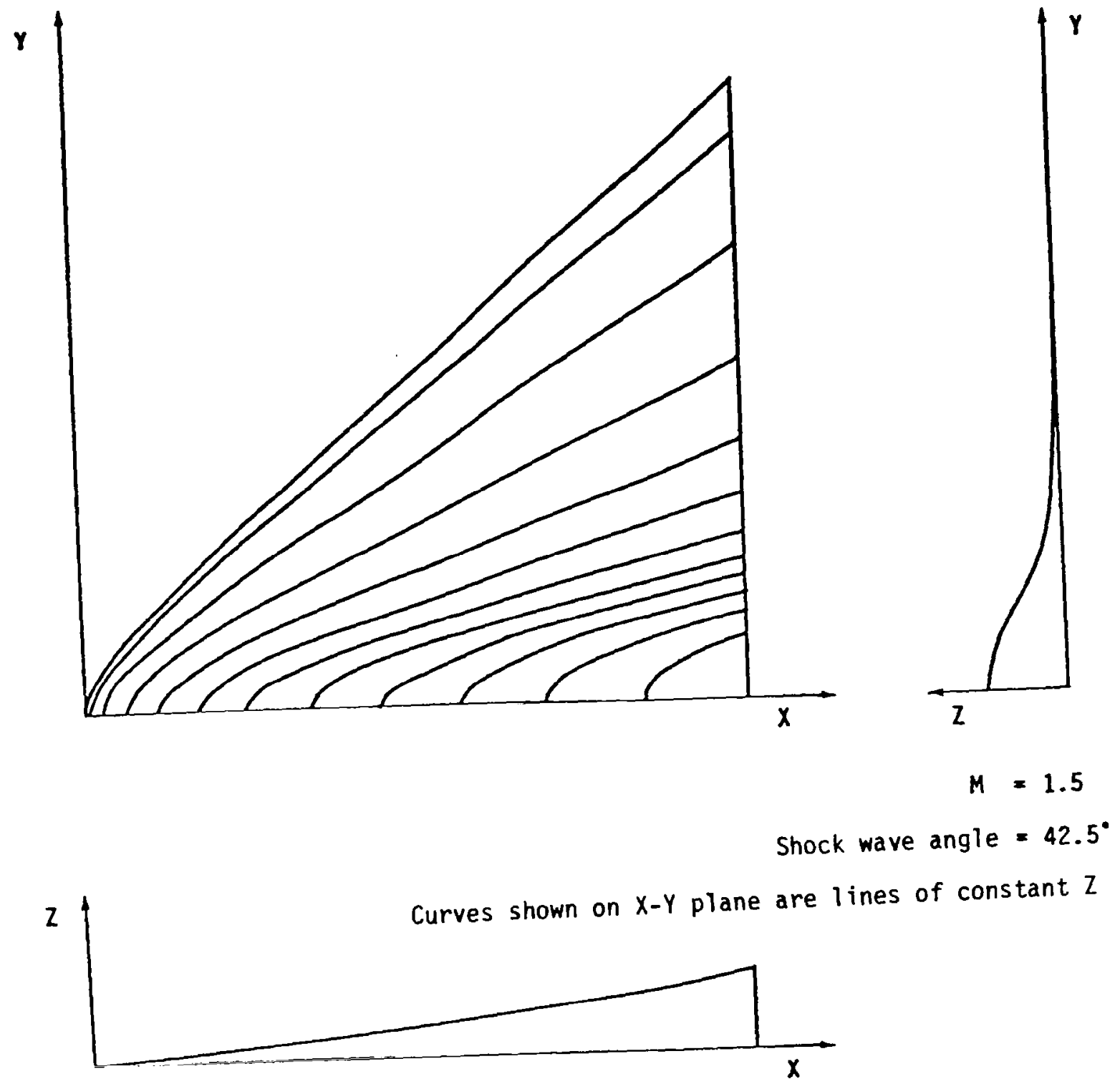

Figure 64.-Geometry of Stream Surface Generated at The Intersection of Conical Shock With Plane Parallel to The Shock Axis 


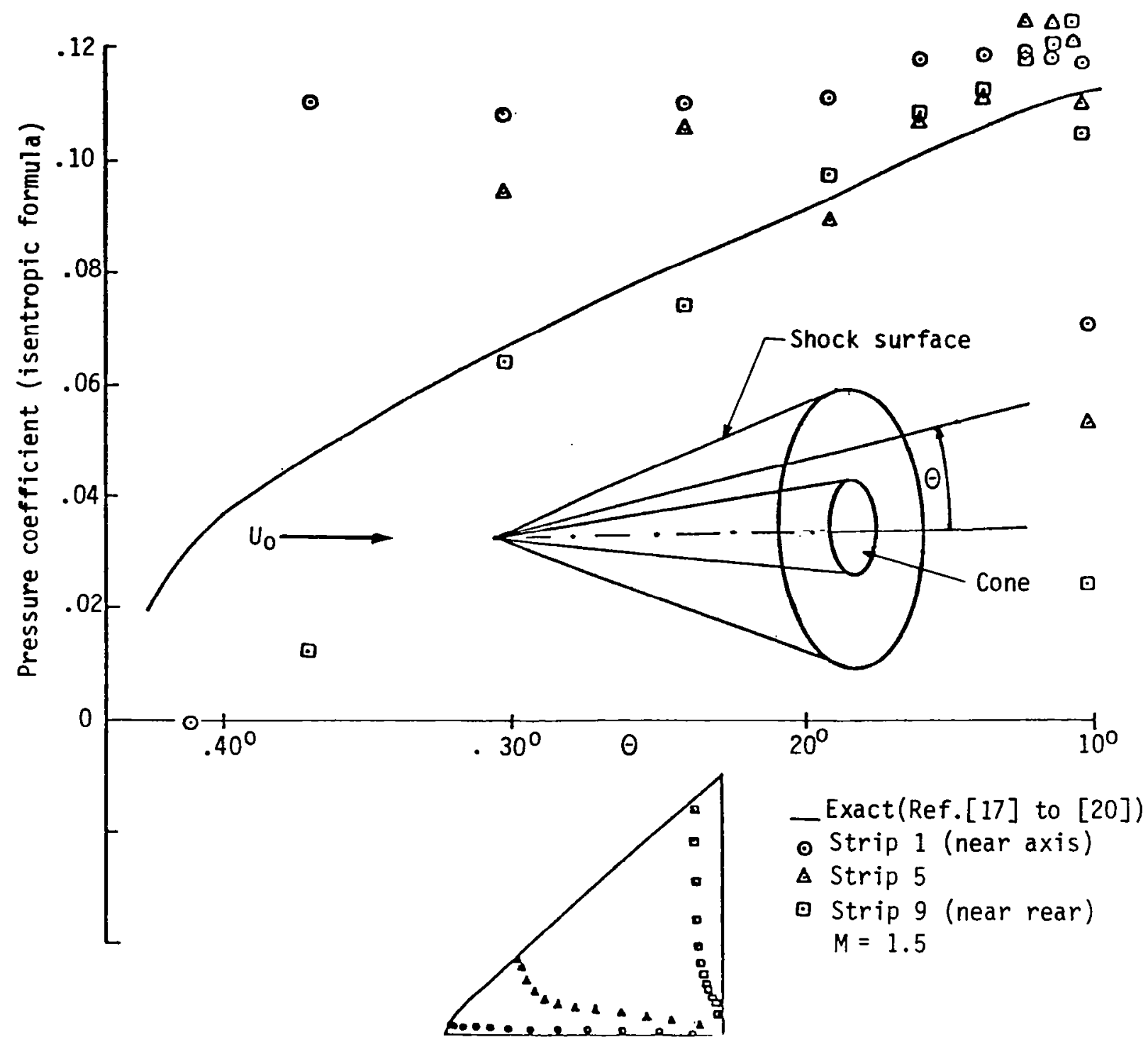

Figure 65.-Pressure Distribution on Upper Surface of Asymmetric Configuration Bounded by Surface of Figure 64 and $x, y$ Plane Using The Nine Parameter Spline With Combined Doublet-source Panels 


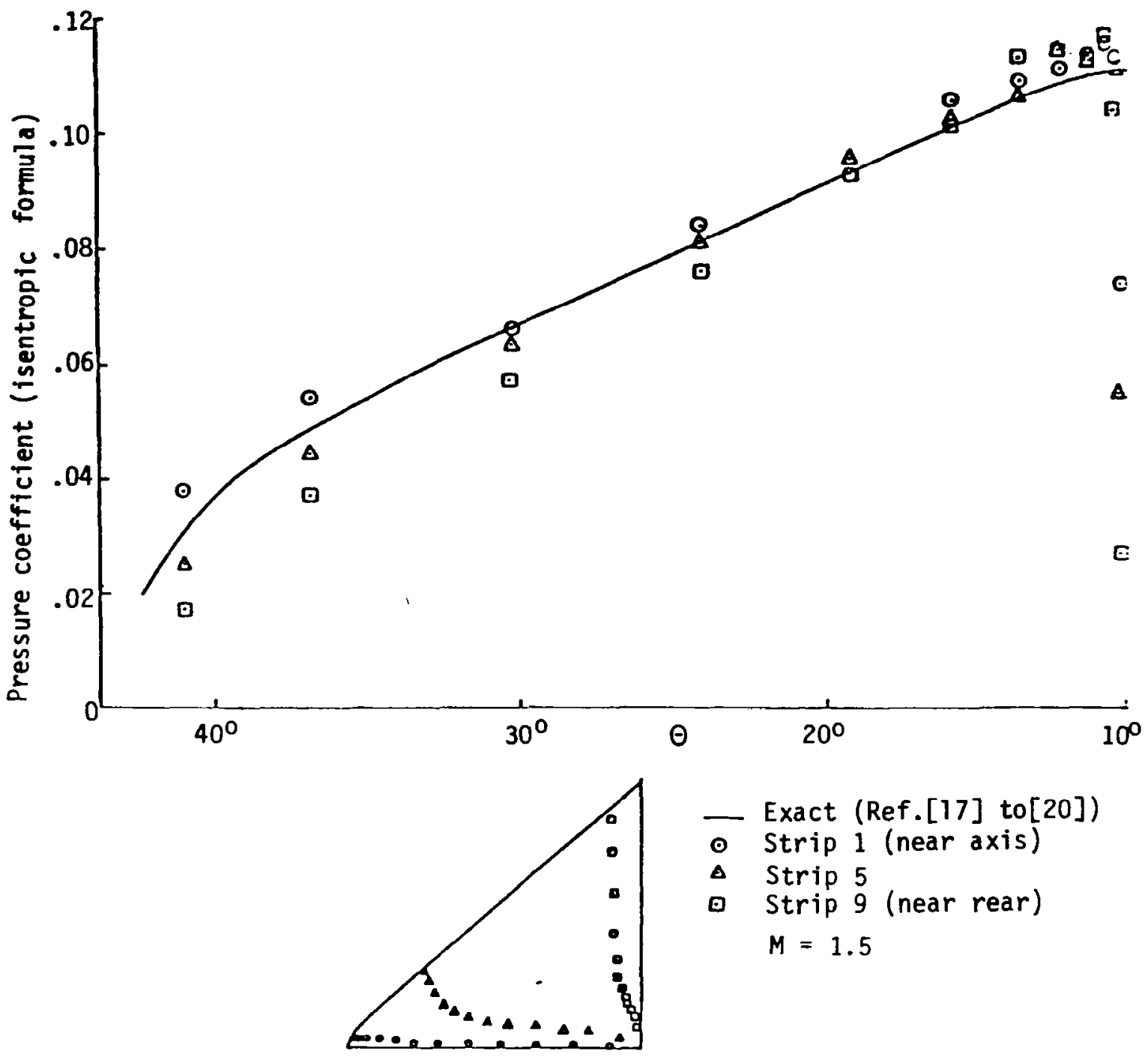

Figure 66.-Pressure Distribution on Upper Surface of Symmetric Configuration Bounded by Surface of Figure 64 And Its Reflection in The $x, y$ Plane Using The Nine Parameter Spline With Combined Doublet-source Panels 


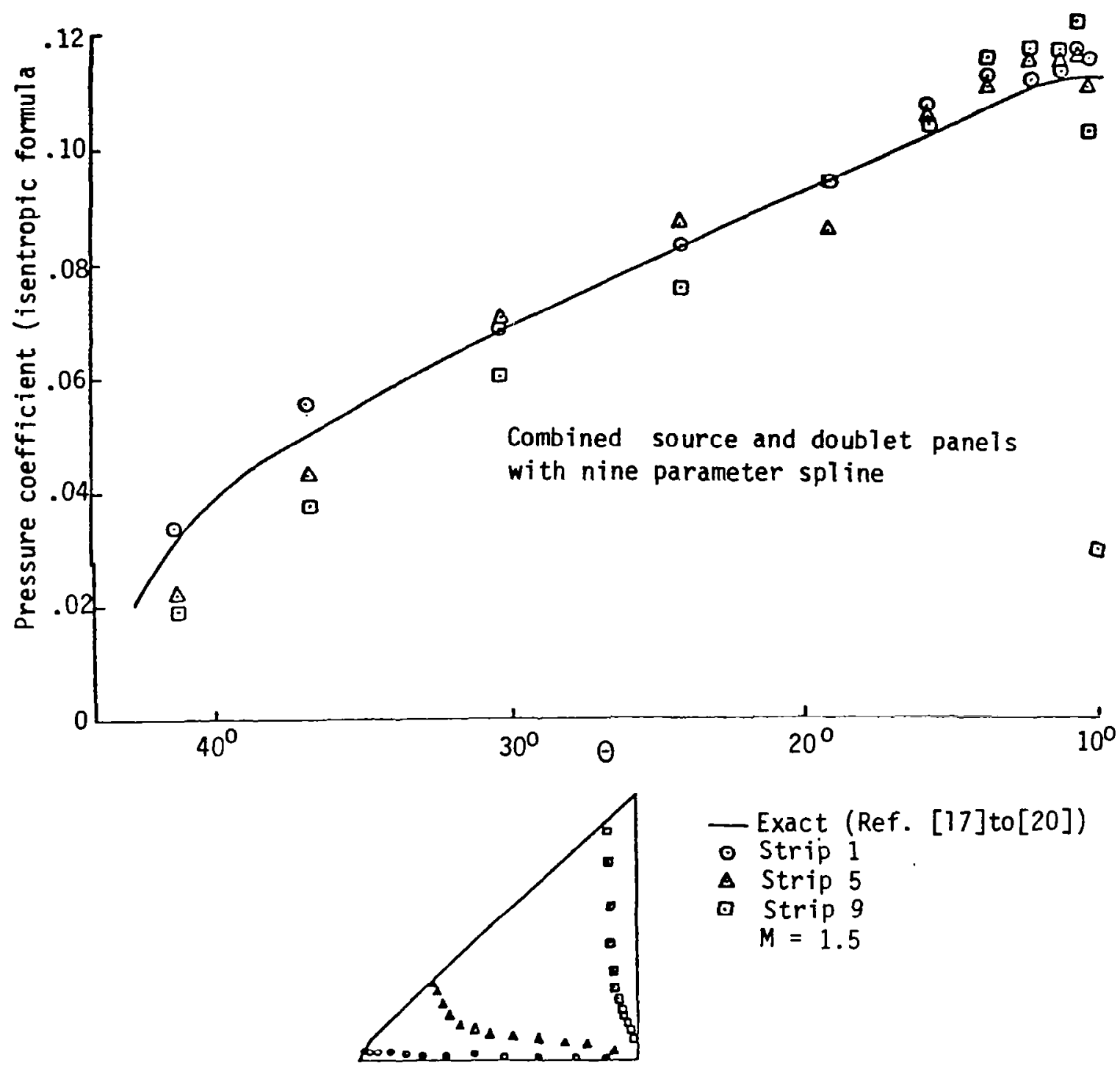

Figure 67.-Pressure Distribution on Upper Surface of Asymmetric Configuration Bounded by Surface of Figure 64 And $x, y$ Plane With Additional Doublets Distributed Over The Mean Surface With Zero Normal Mass Flux Boundary Conditions 


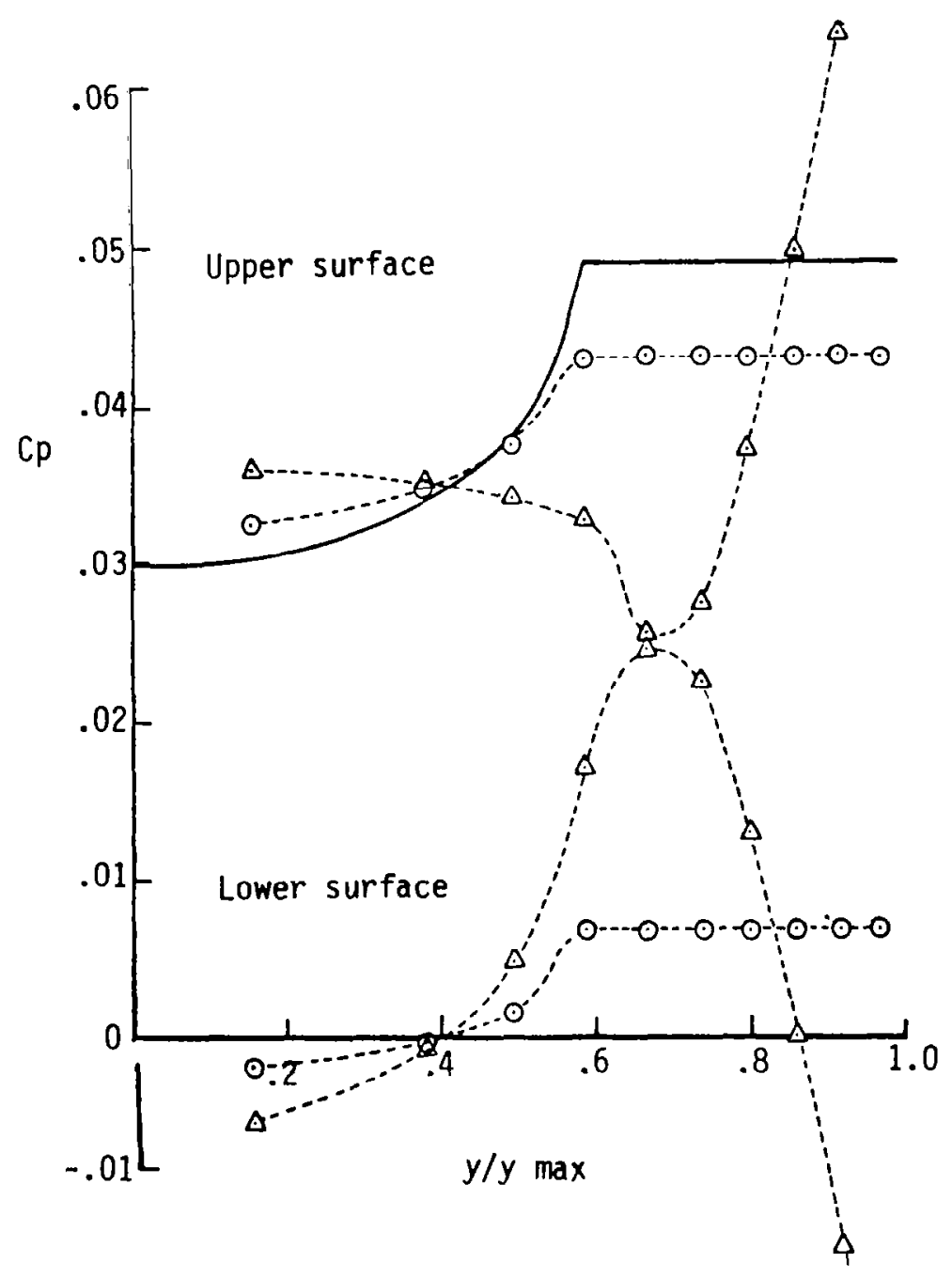

\section{Discontinuous $\mu$}

Linear theory (Ref.[23])

$$
\begin{aligned}
& \odot \quad x=.0056 \\
& \Delta \quad x=.9 C_{0}
\end{aligned}
$$

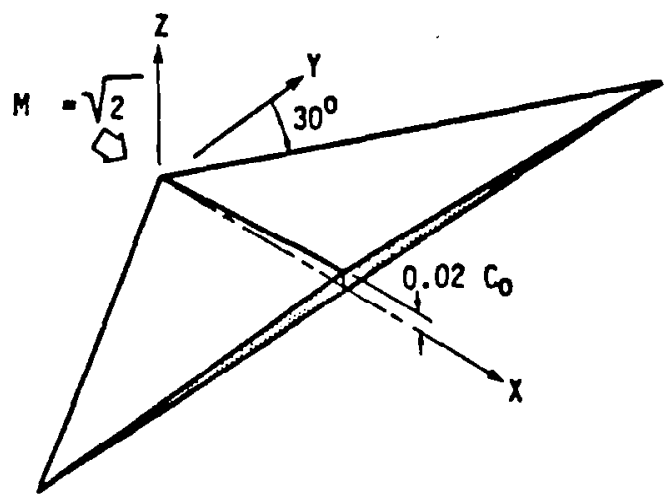

Lower surface is at zero angle of attack

Figure 68. - Pressure Distribution Near Leading And Trailing Edge of Delta Wing With Sharp Supersonic Leading Edge Using The Nine Parameter Doublet Spline With Combined Doublet-source Pane/s 


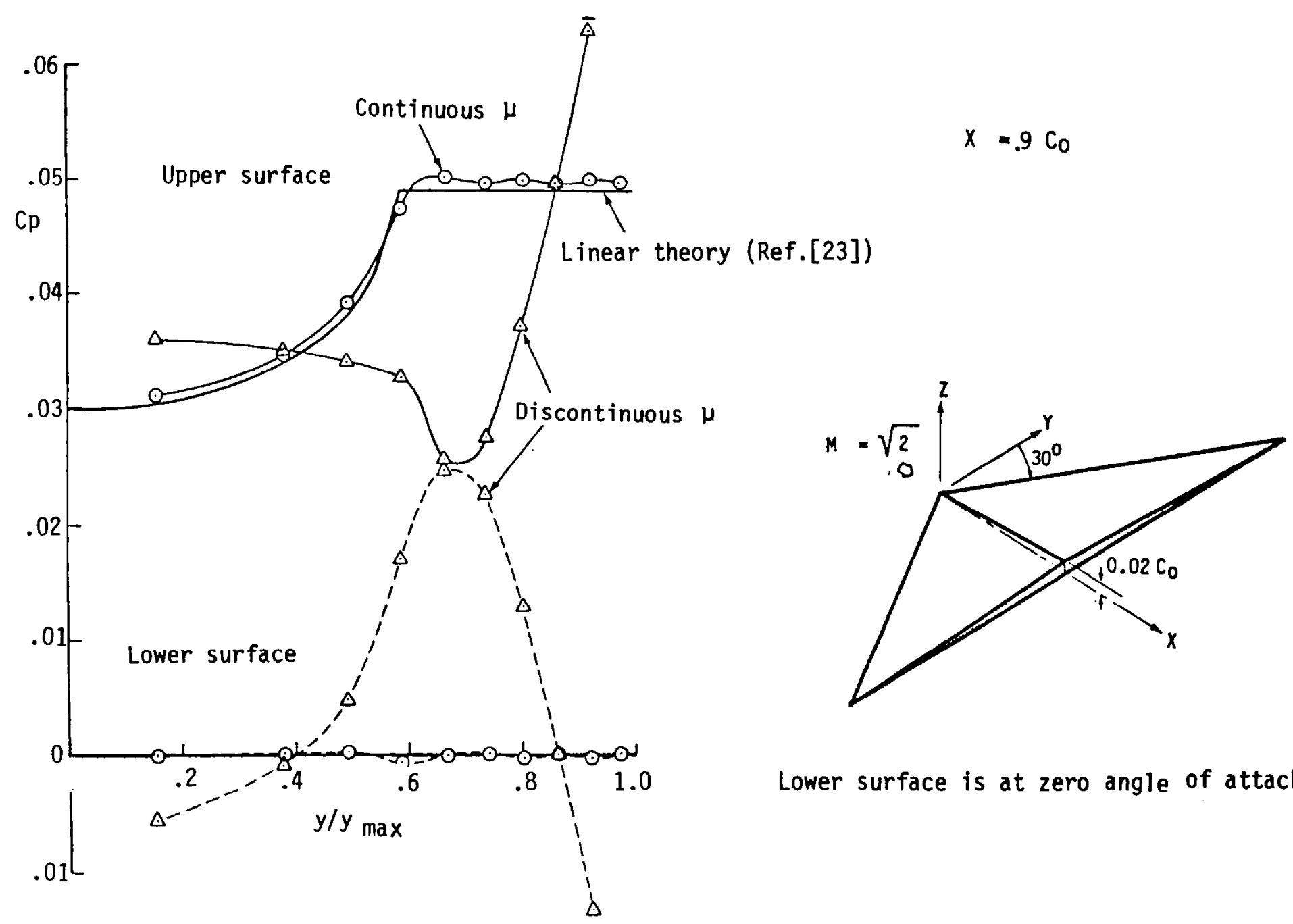

Figure 69. - Pressure Distribution Near Trailing Edge of Delta Wing With Sharp Supersonic Leading Edge Using The Nine Parameter Spline With Combined Doublet-source Pane/s 


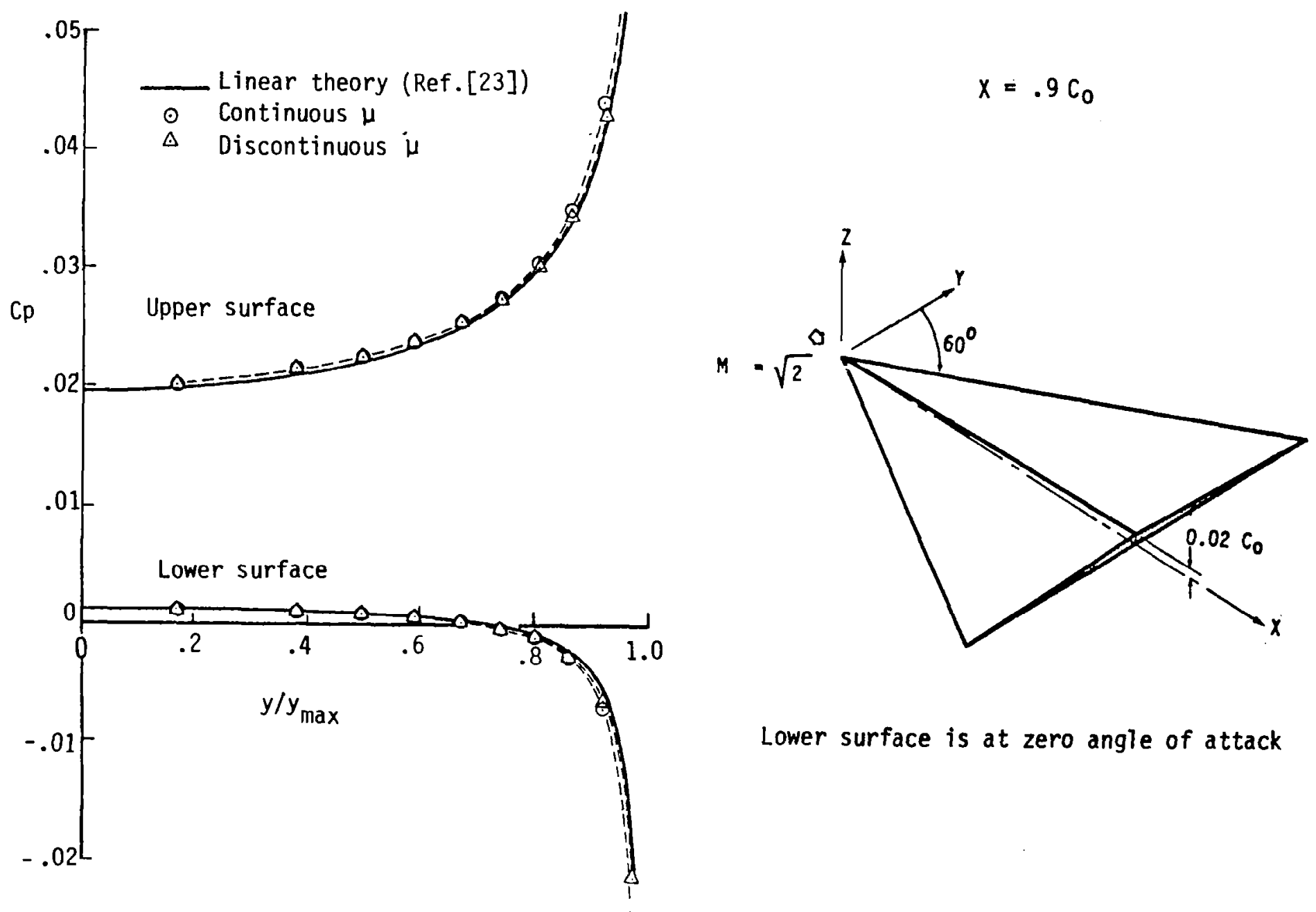

Figure 70. - Pressure Distribution Near Trailing-Edge of Delta Wing With Sharp Subsonic Leading Edge Using The Nine Parameter Spline With Combined Doublet-source 


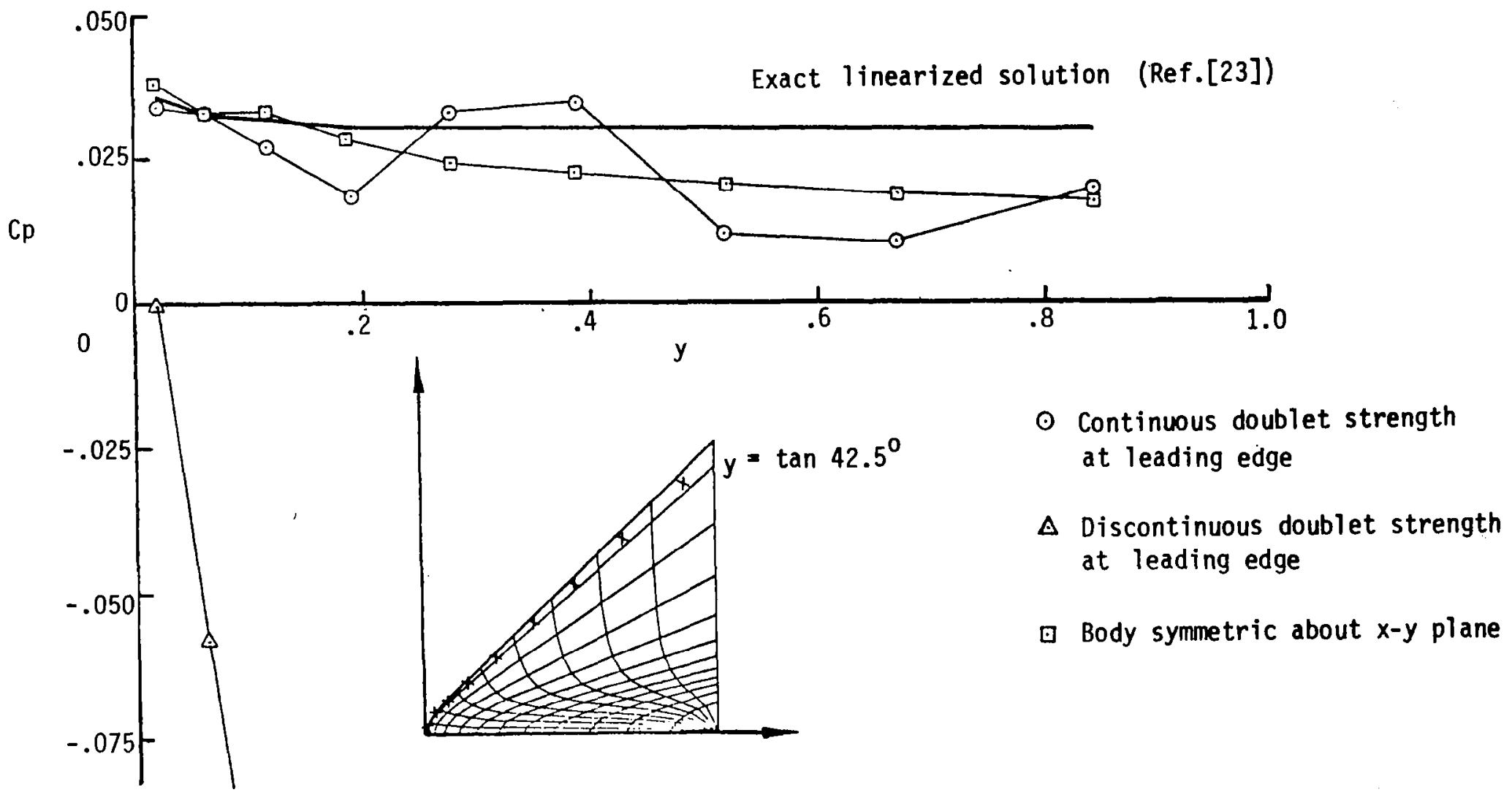

Figure 71.--Pressure Distribution Near Leading Edge of Conical Flow Stream Surface Using The Nine Parameter Spline With Combined Doublet-source Panels 

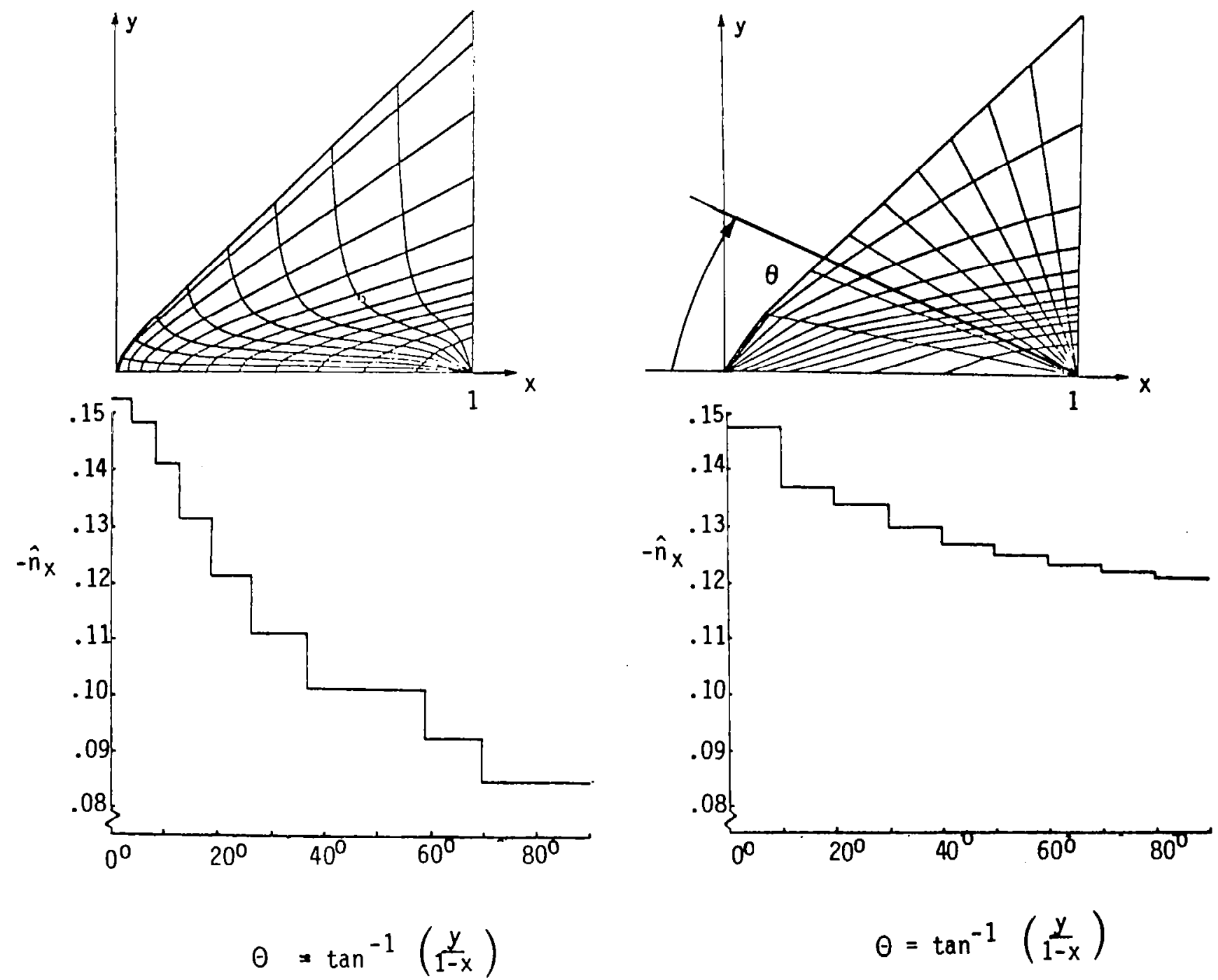
Panels of The Conical Stream Surface 


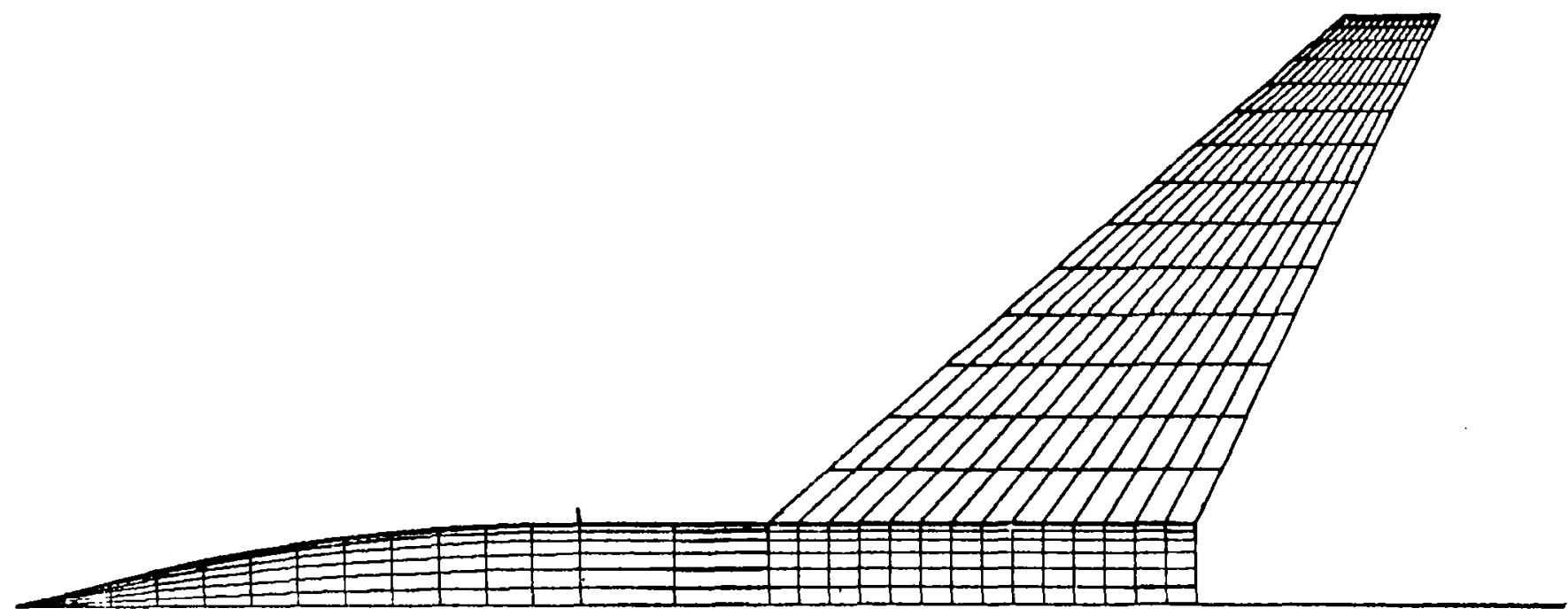

Figure 73. - Paneling on Wing/Body of NASA Memo 10-15-58L With Two Axes of Symmetry 


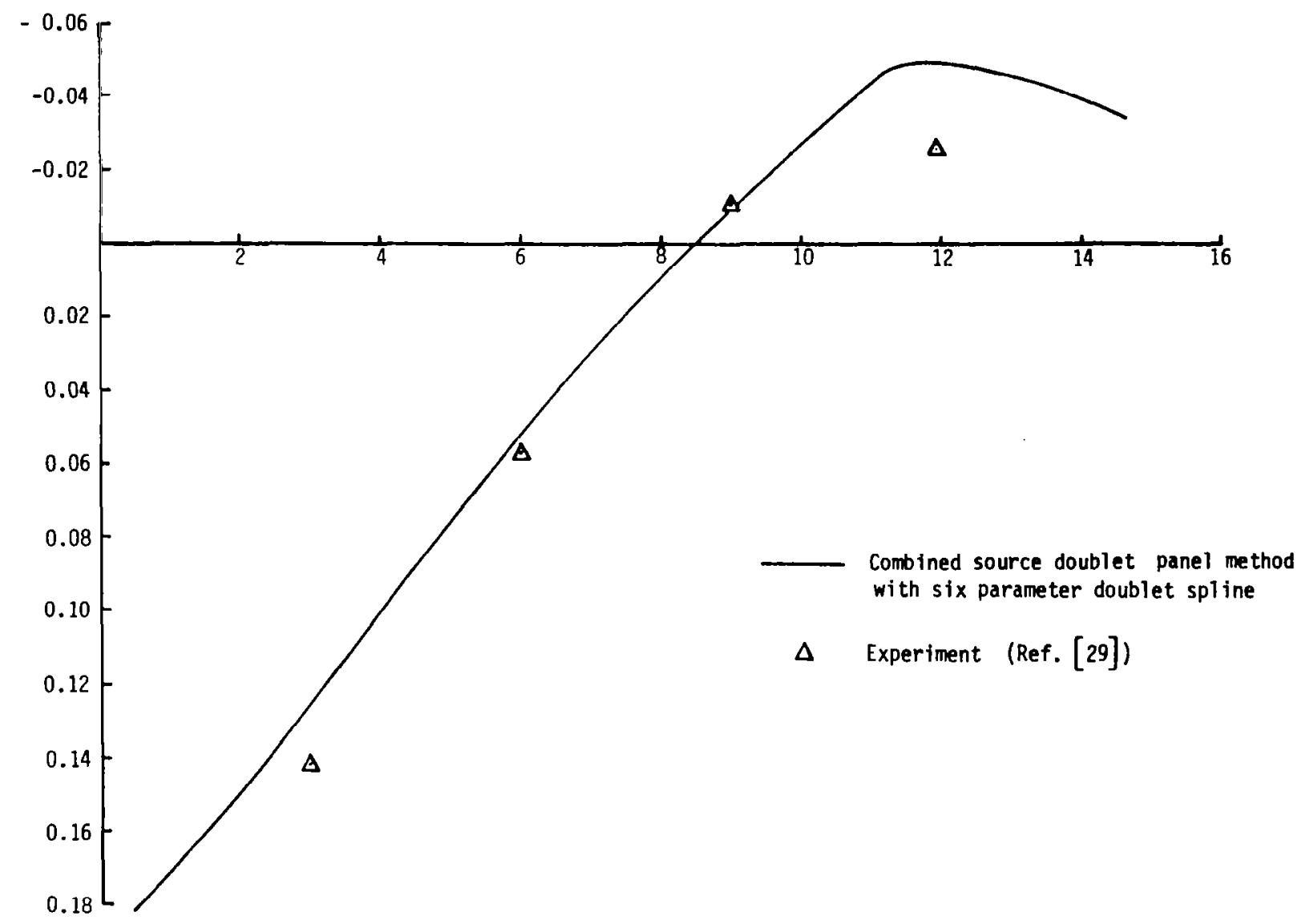

Figure 74.-Pressure Distribution Along Top of Body on Wing/body of NASA Memo 10-15-58L 

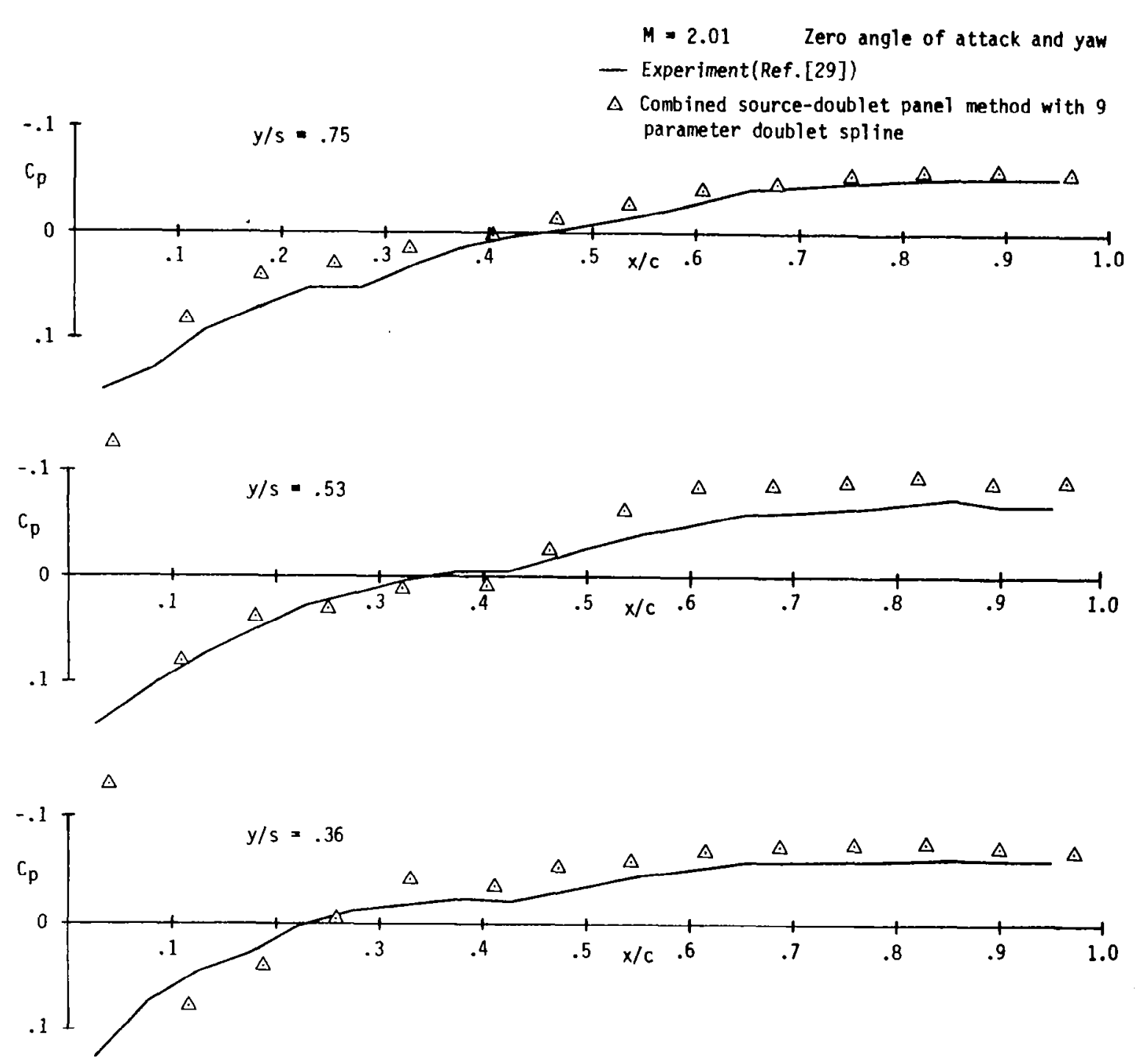

Figure 75.-Pressure Distribution on The Wing of The Symmetrical Wing/body of NASA Memo 10-15-58L 


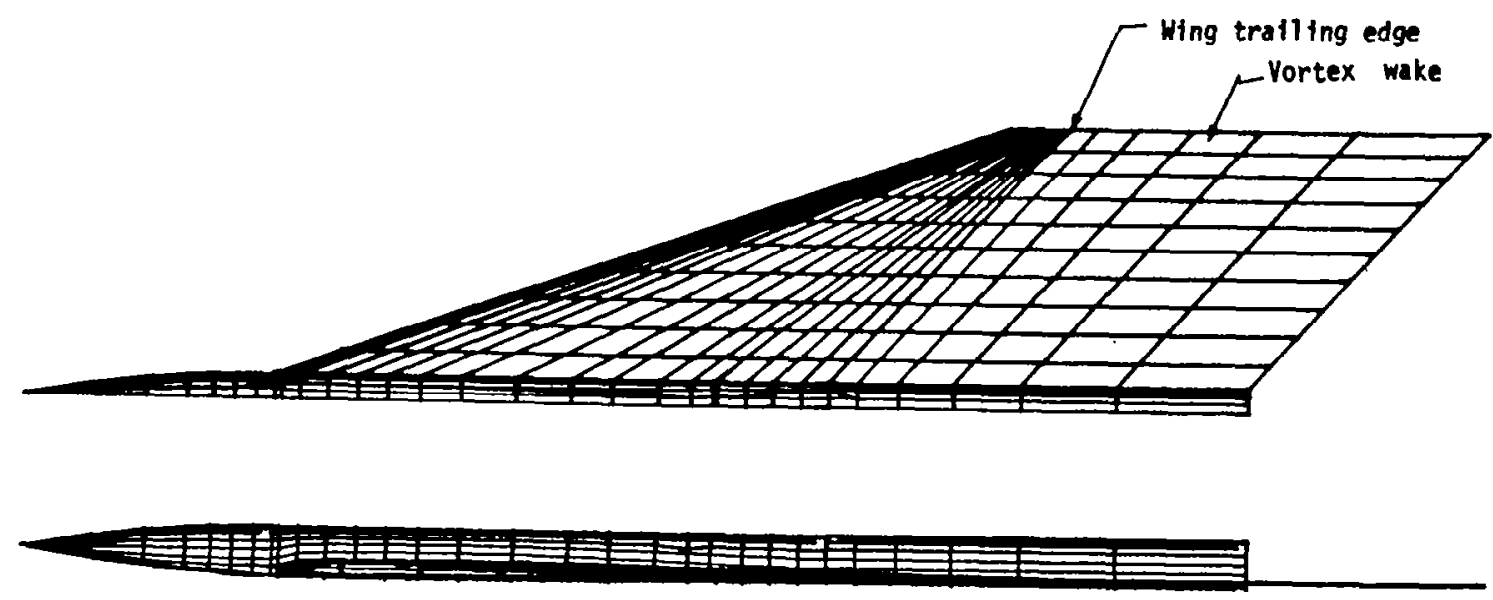

Figure 76.-Paneling on The Arrow Wing/body With The Straight Wing 
Combined source and 9 parameter doublet spline
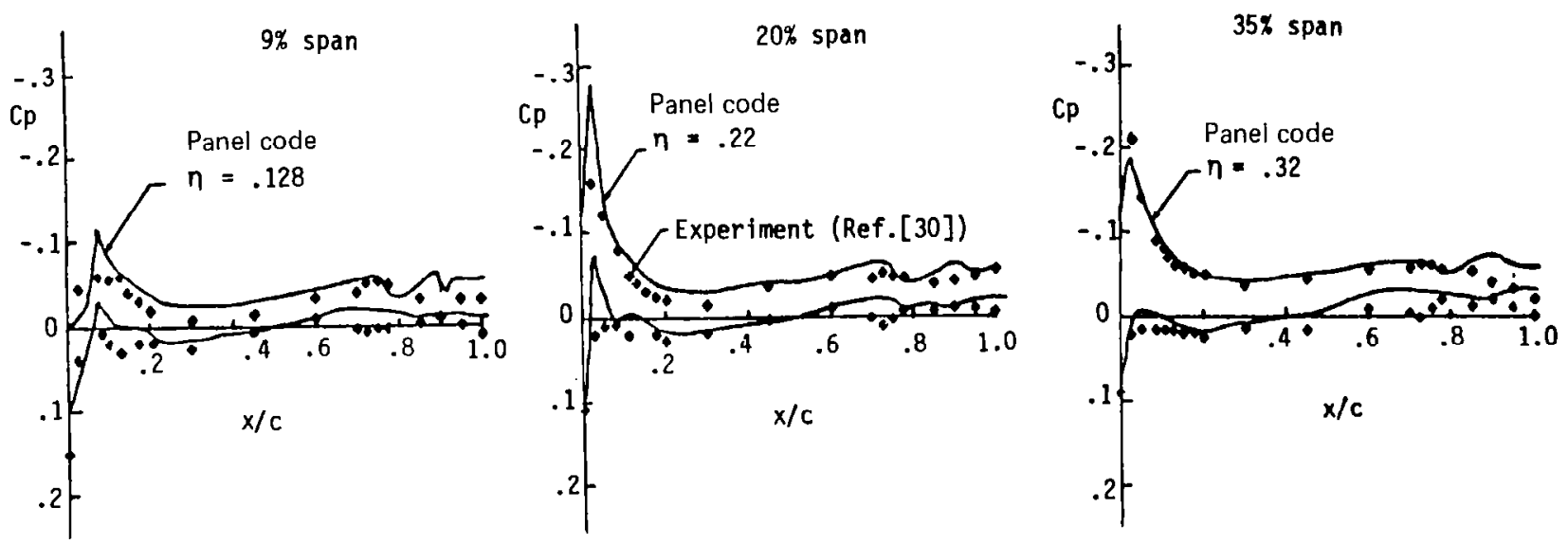

Figure 77.- Pressure Distribution on The Wing of The Arrow Wing/body of Figure 76 With Experimental Values at 9\%, 20\%, and 35\% Span Locations and Computed Results at $12.8 \%, 22 \%$, and $32 \%$ Span Locations 

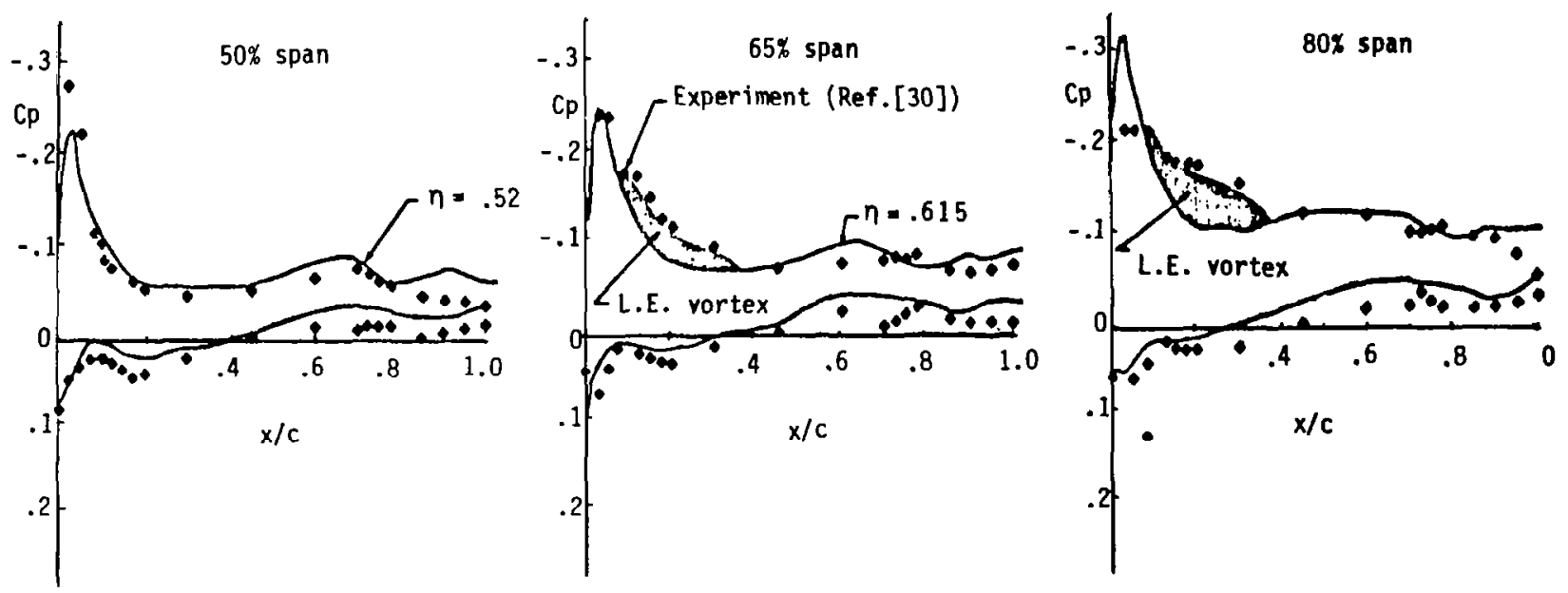

Figure 78.-Pressure Distributions on Wing of The Arrow Wing/body of Figure 76 With Experimental Values at 50\%, 65\%, And 80\% Span Locations And Computed Results at $52 \%, 61.5 \%$, and $80 \%$ Span Locations 


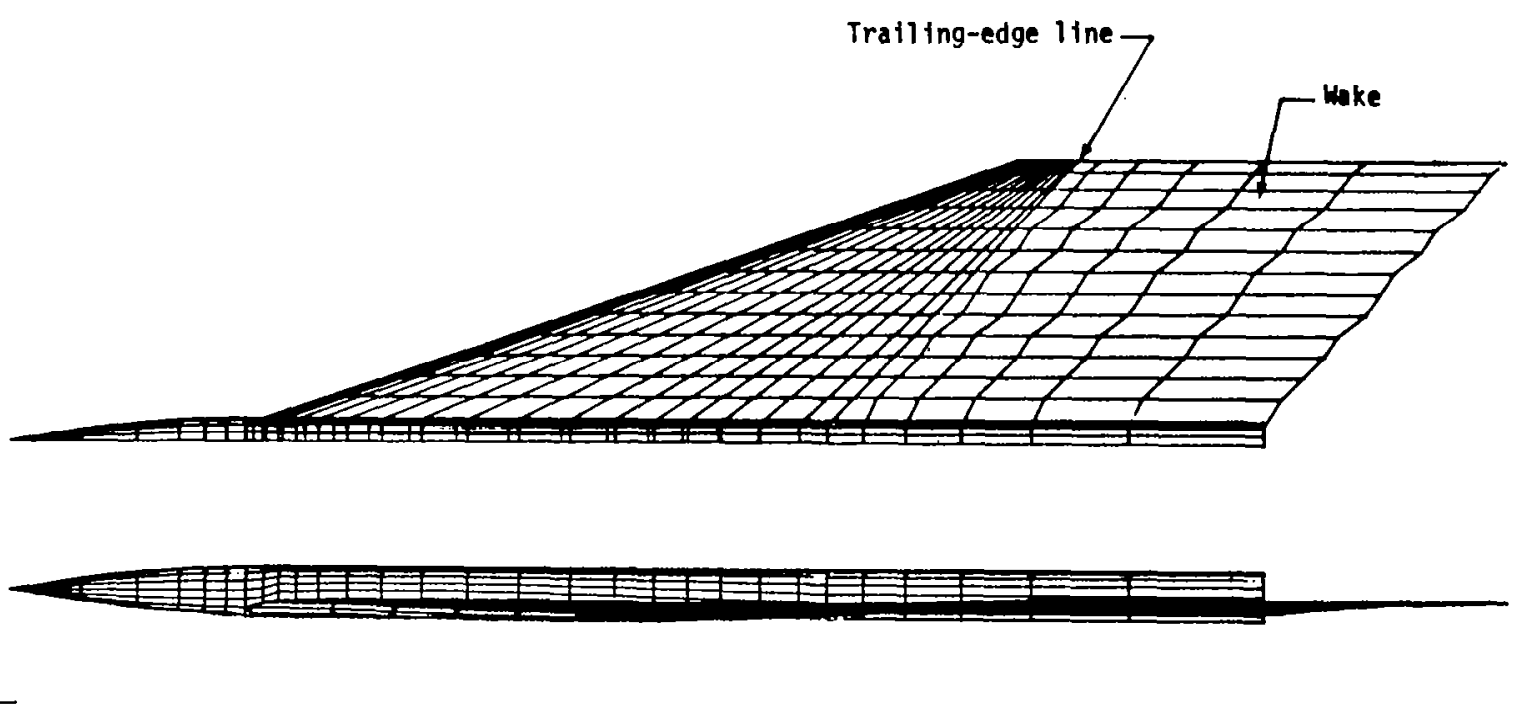

Figure 79.-Paneling on The Arrow Wing/body With The Twisted Wing 
Combined source and 9 parameter

$M=1.7$ doublet spline

$a=4^{0}$
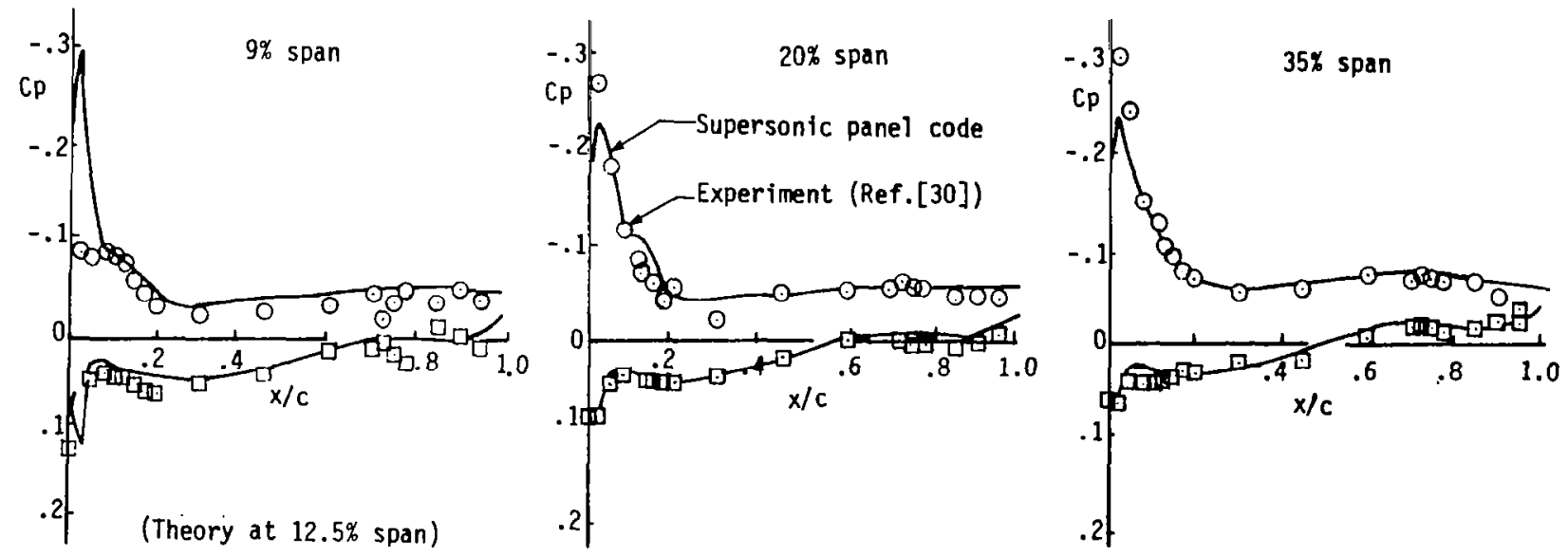

Figure 80.-Pressure Distributions on The Twisted Arrow Wing/body Combination of Figure 79 at 9\%, 20\%, And 35\% of Span Locations 
Combined source and 9 parameter doublet spline
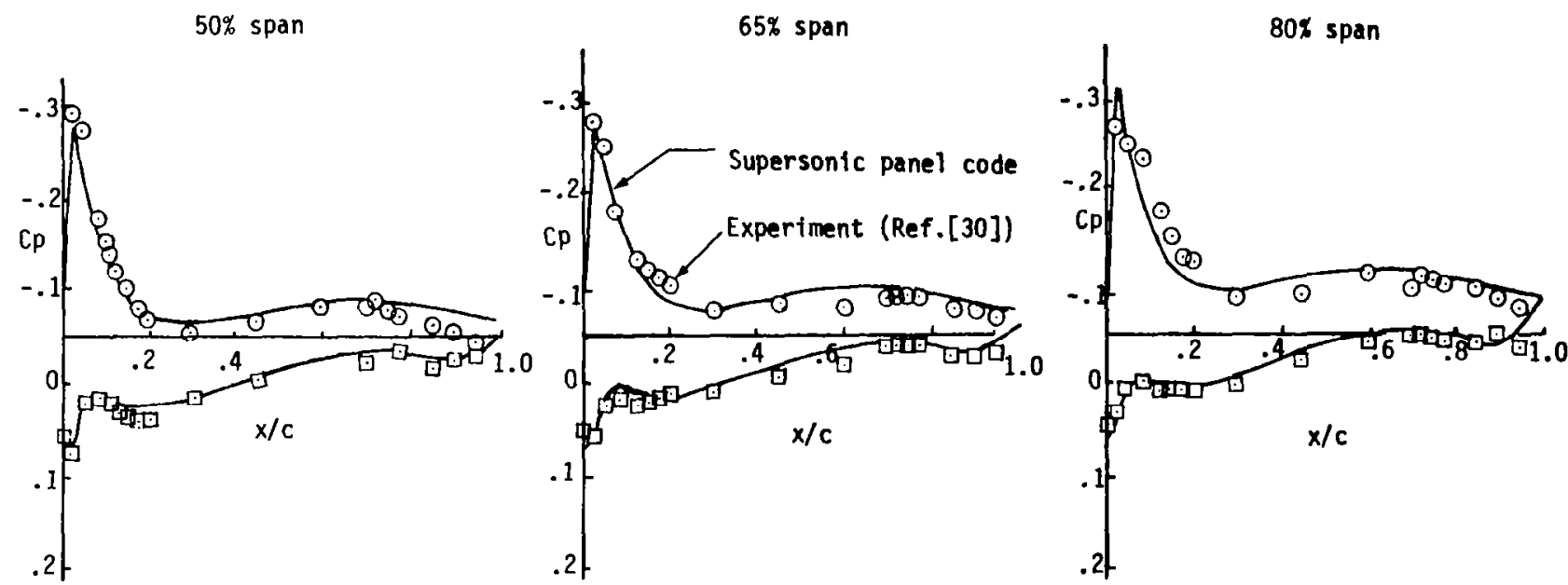

Figure 81.-Pressure Distributions on The Twisted Arrow Wing/body Combination of Figure 79 at 50\%, 65\%, and $80 \%$ Span Locations 

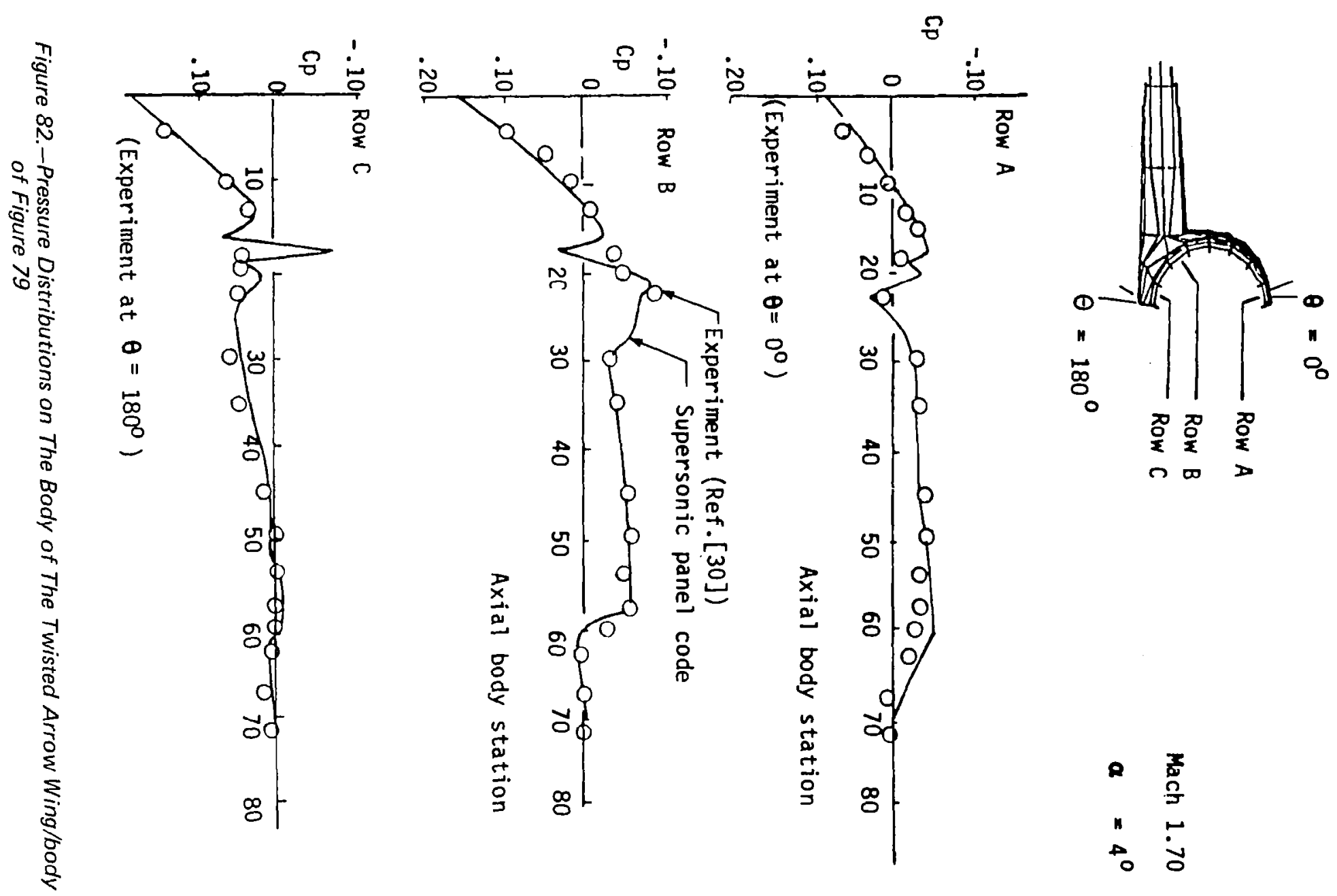

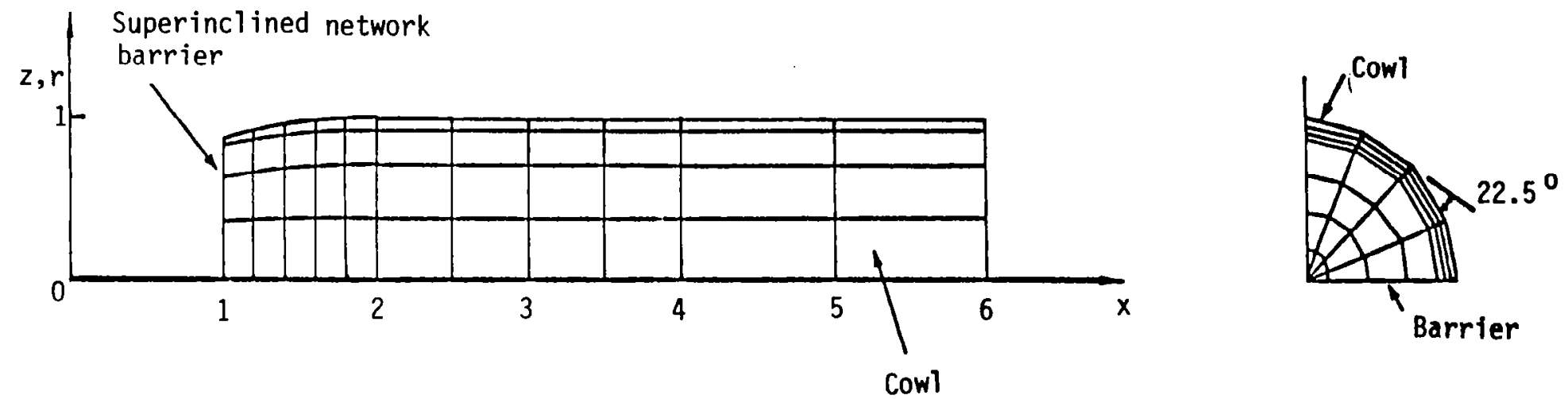

Figure 83.-Example of Superinclined Network to Close Inlet on a Nacelle 

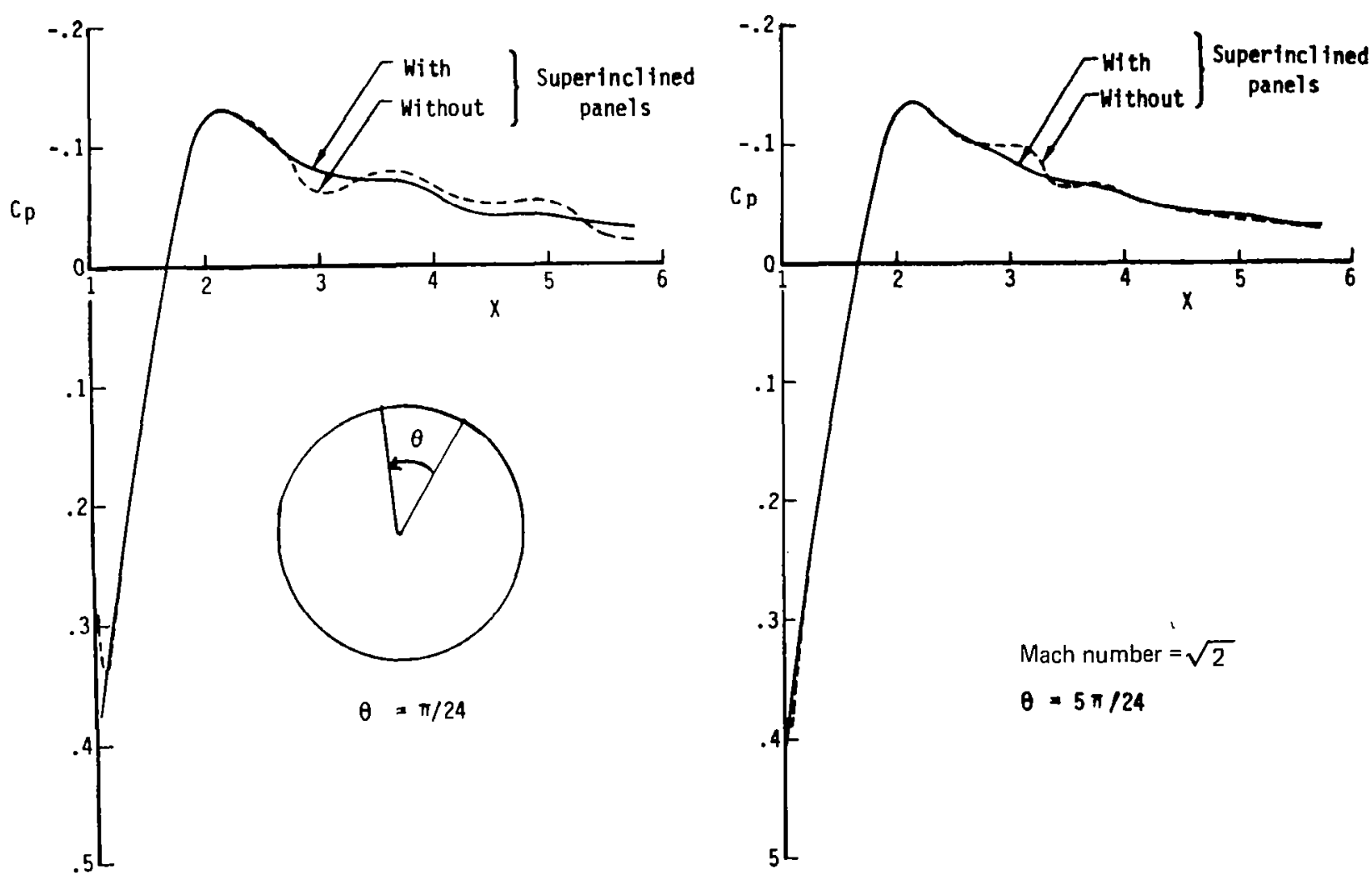

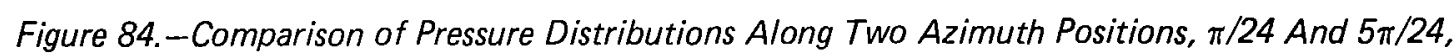
on The Exterior Surface of The Nacelle For The Flows Calculated With And Without Superinclined Network in The Presence of a Perturbation From Constant Upstream Source Panels 

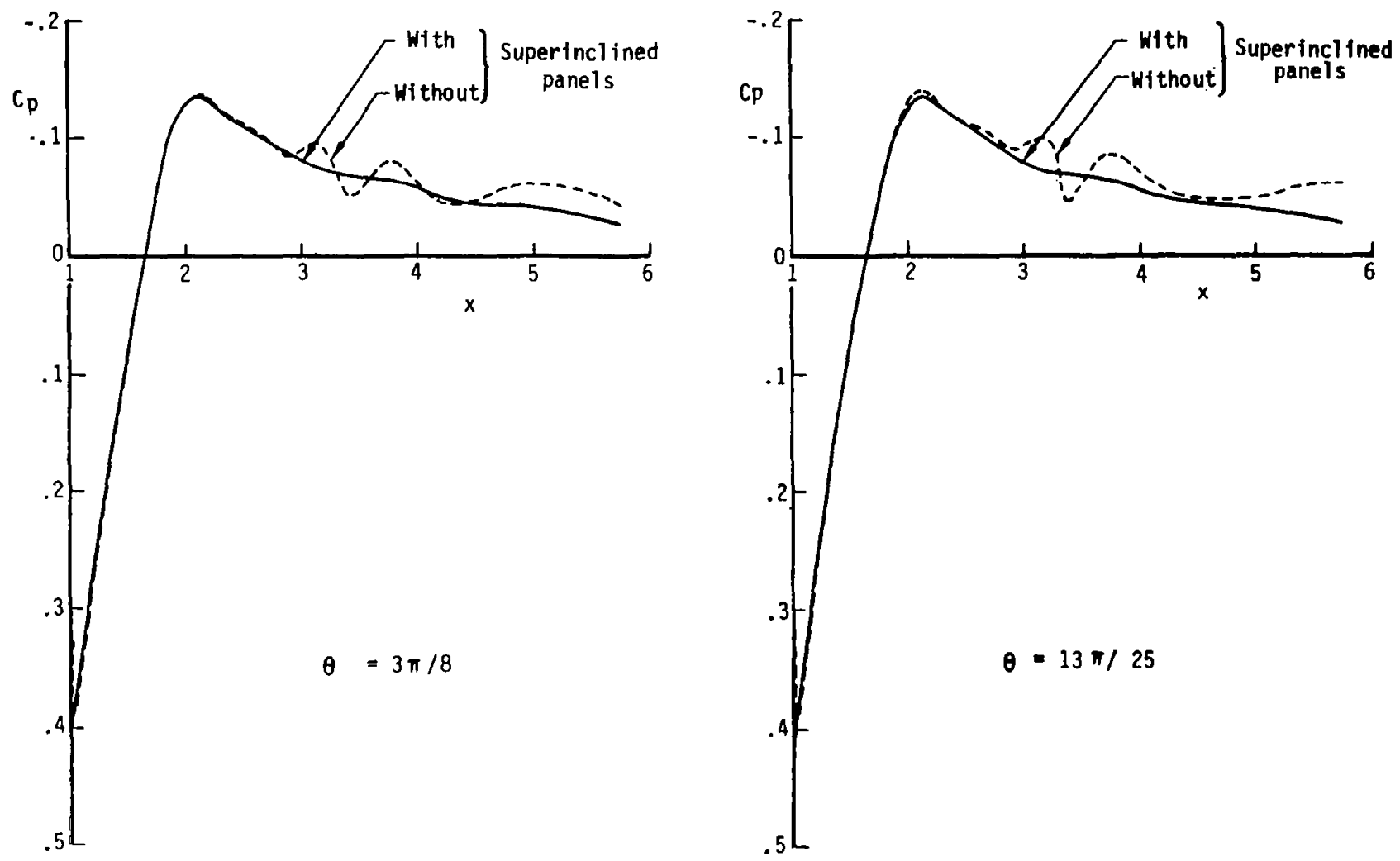

Figure 85. - Comparison of Pressure Distributions Along Two Azimuth Positions, $3 \pi / 8$ And $13 \pi / 25$, on The Exterior Surface of The Nacelle For The Flows Calculated With And Without Superinclined Network in The Presence of a Perturbation From Constant Upstream Pane/s 

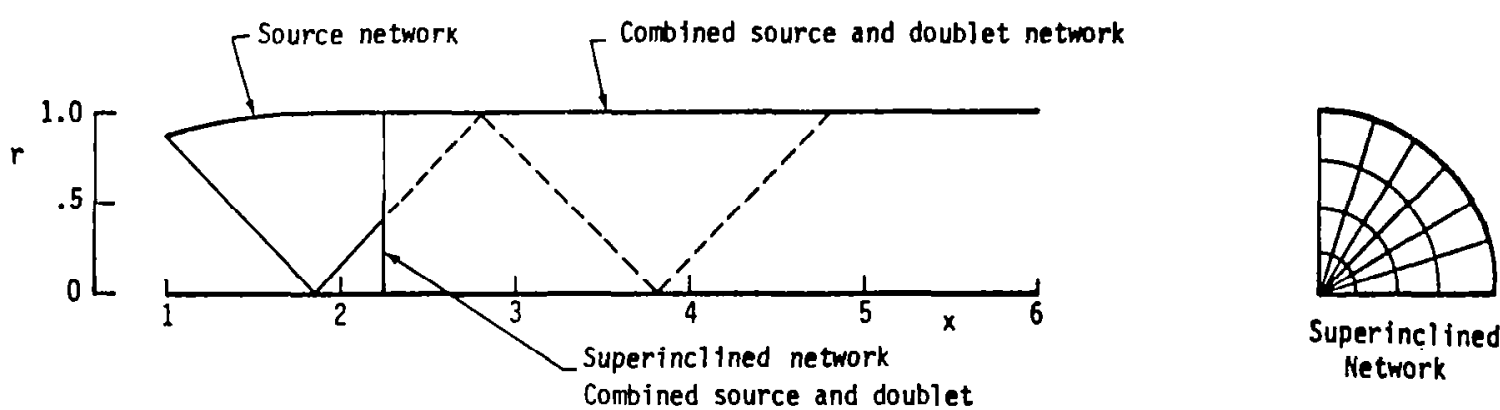

Superinclined Network

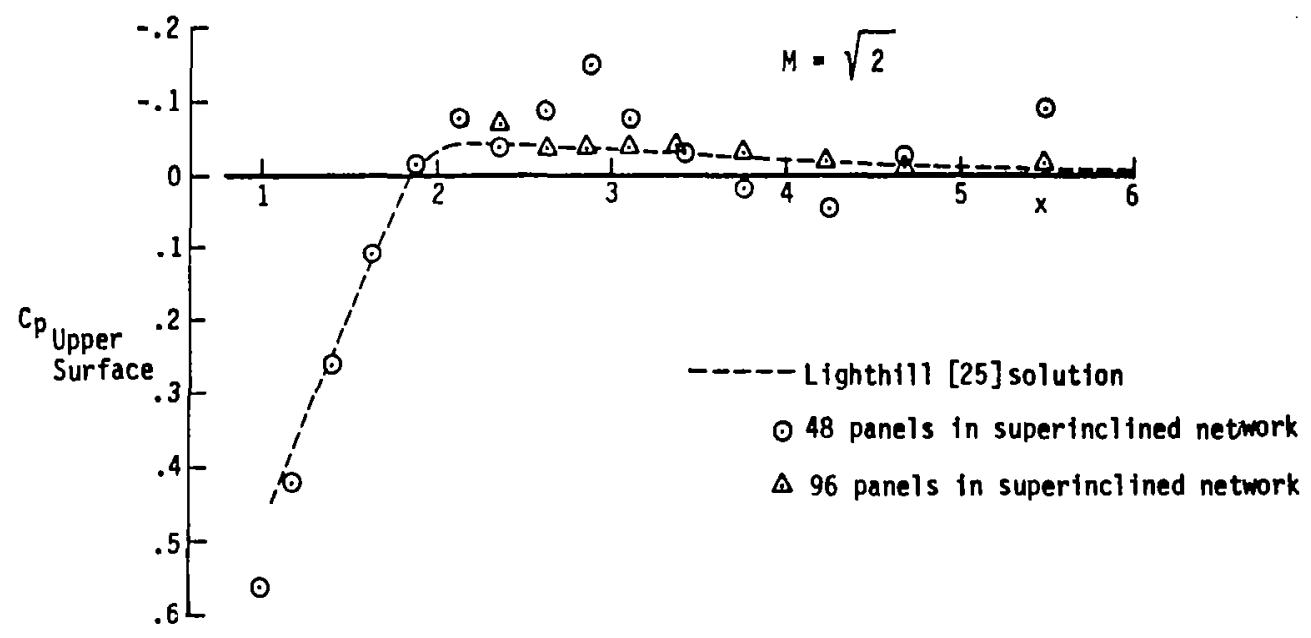

Figure 86. - Comparison of Exterior Pressure Distribution on The Exterior Surface of The Nacelle Containing an Interior Superinclined Network With The Lighthill Solution 

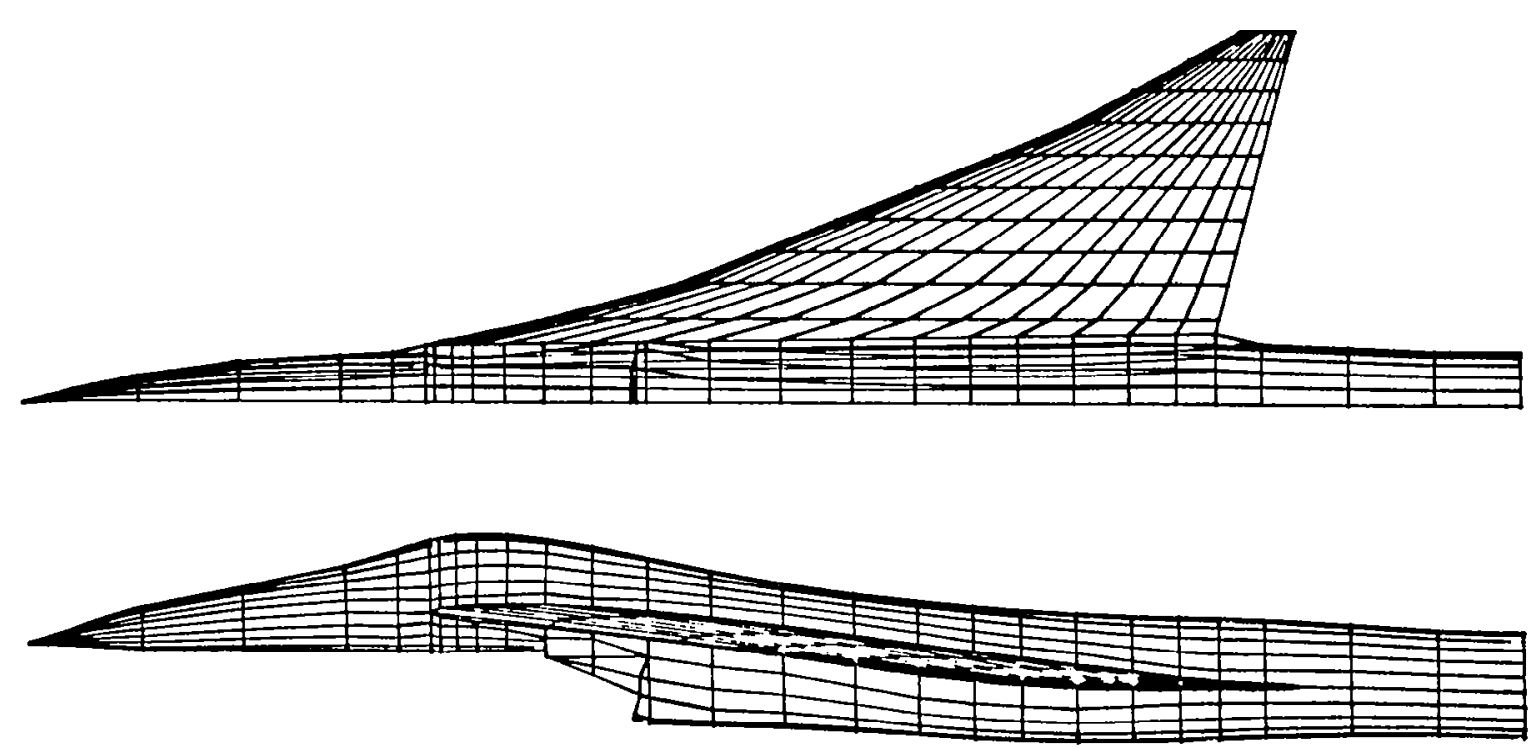

Figure 87.-Top And Side Views of Paneling on The LES 216 Supercruiser 


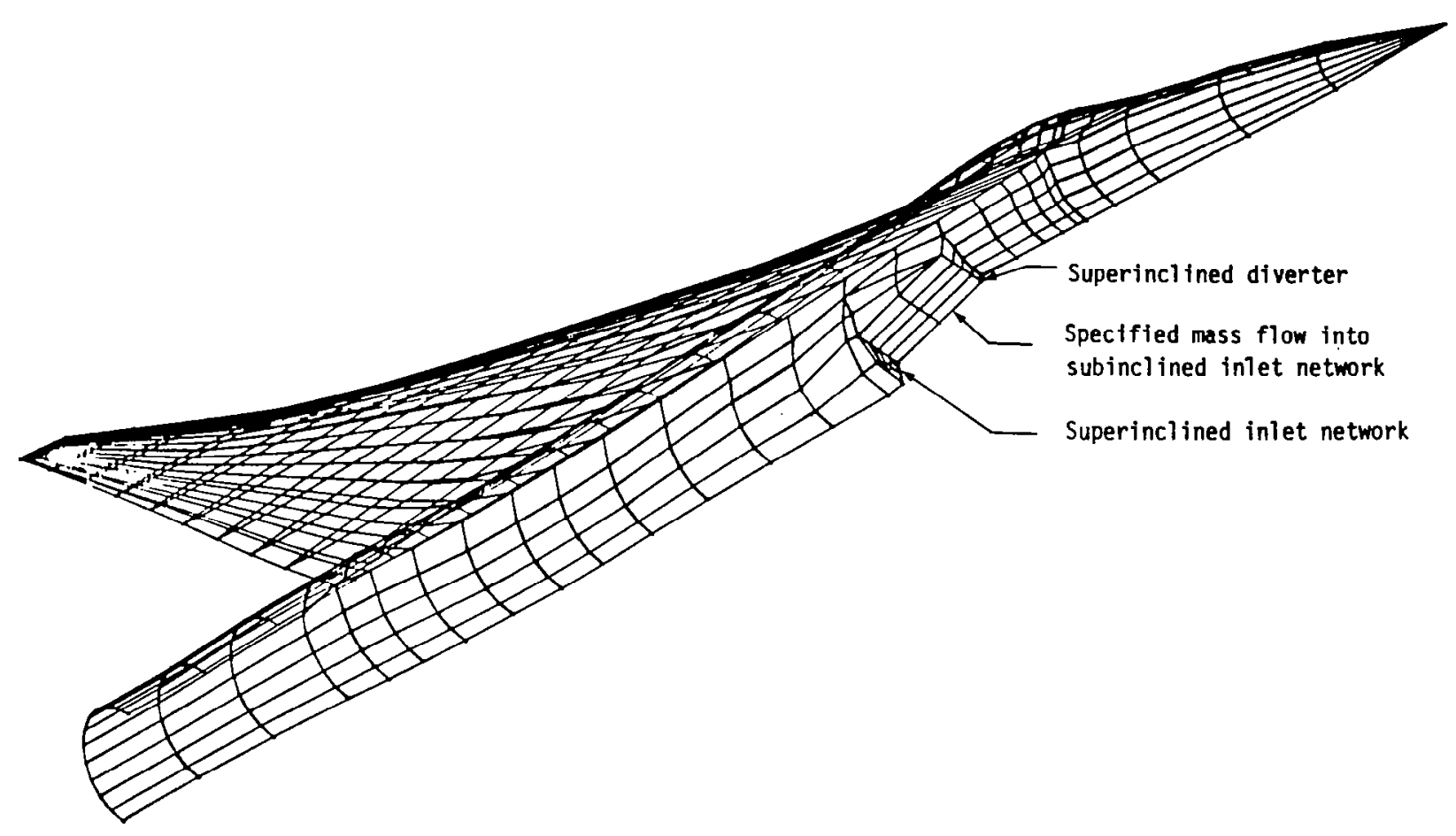

Figure 88.-Perspective View of The Paneling on The LES 216 Supercruiser 

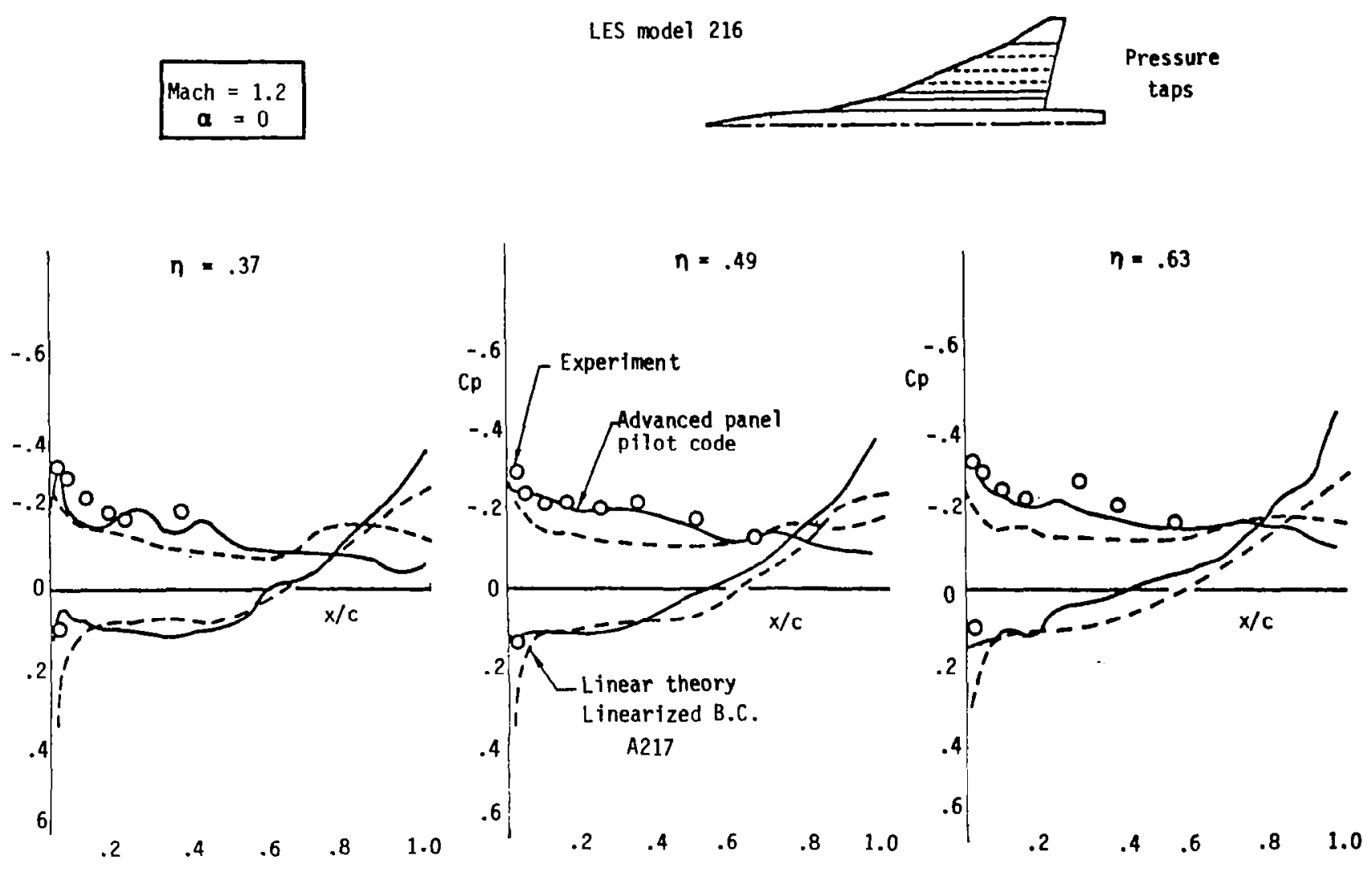

Figure 89. -Wing Pressure Distributions on The LES 216 Supercruiser 


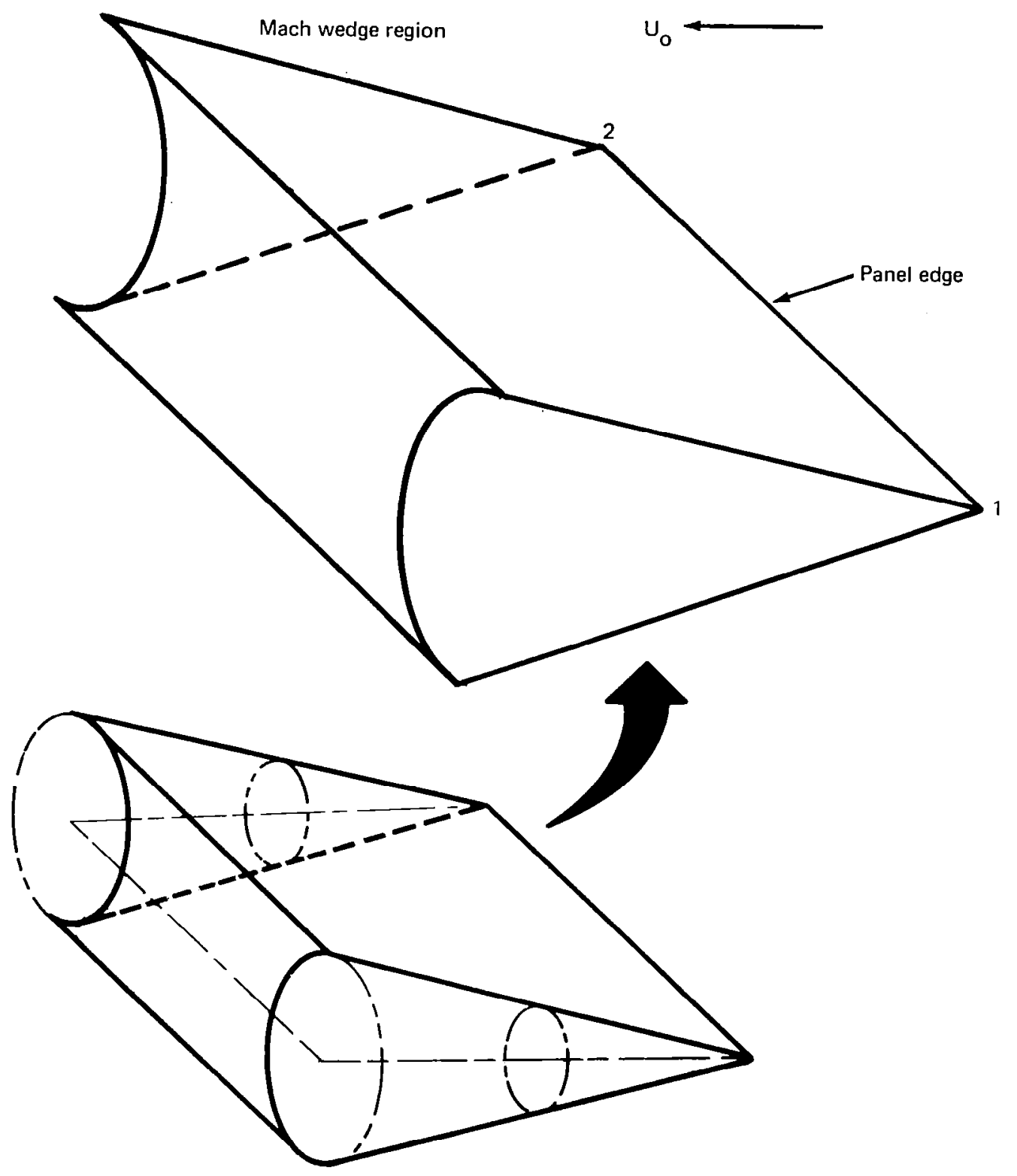

Figure 90.-Mach Wedge Region Behind Supersonic Panel Edge 


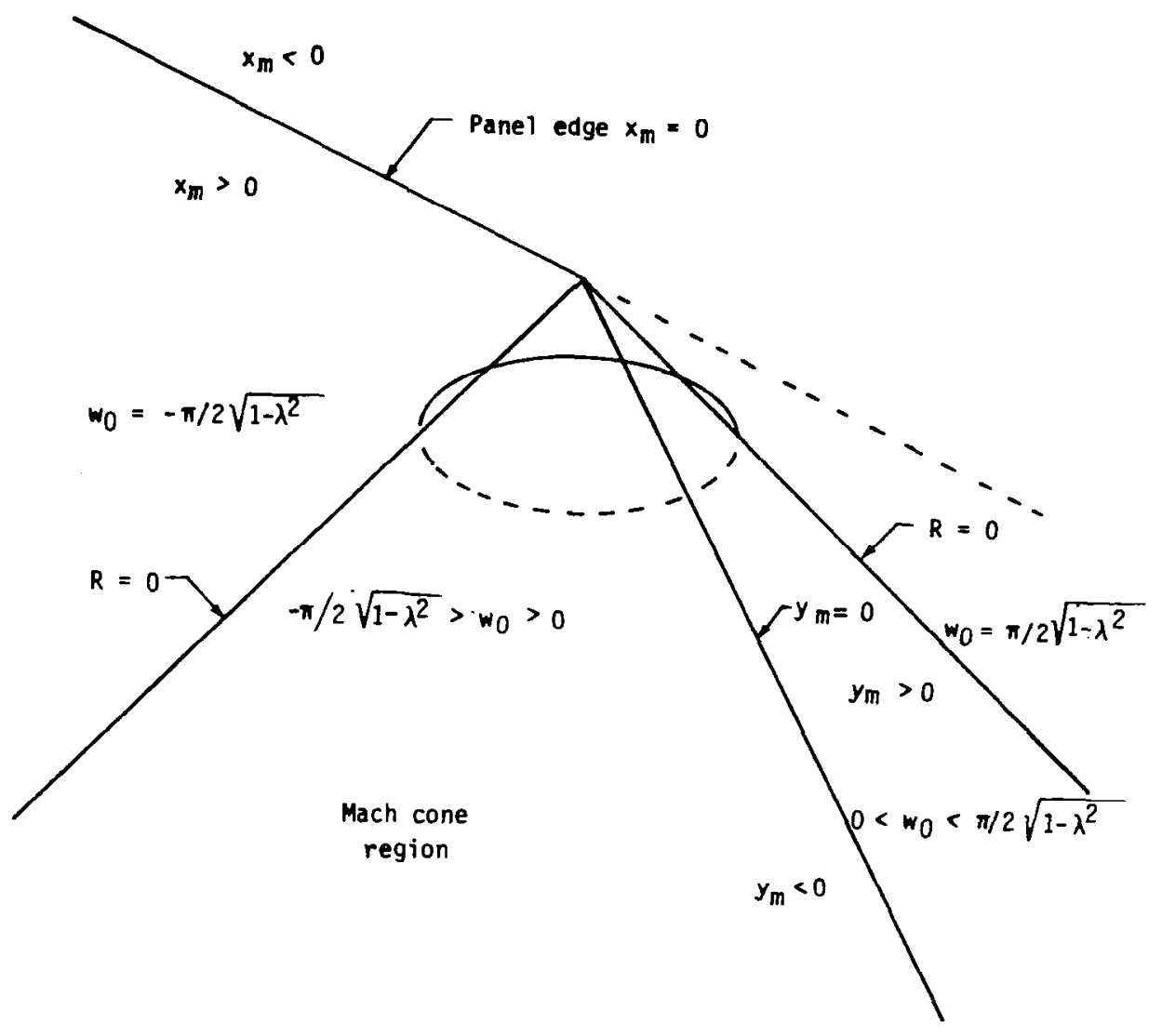

Figure 91.-Evaluation of $w_{O}$ For One Edge Endpoint at Points on The Panel Plane 


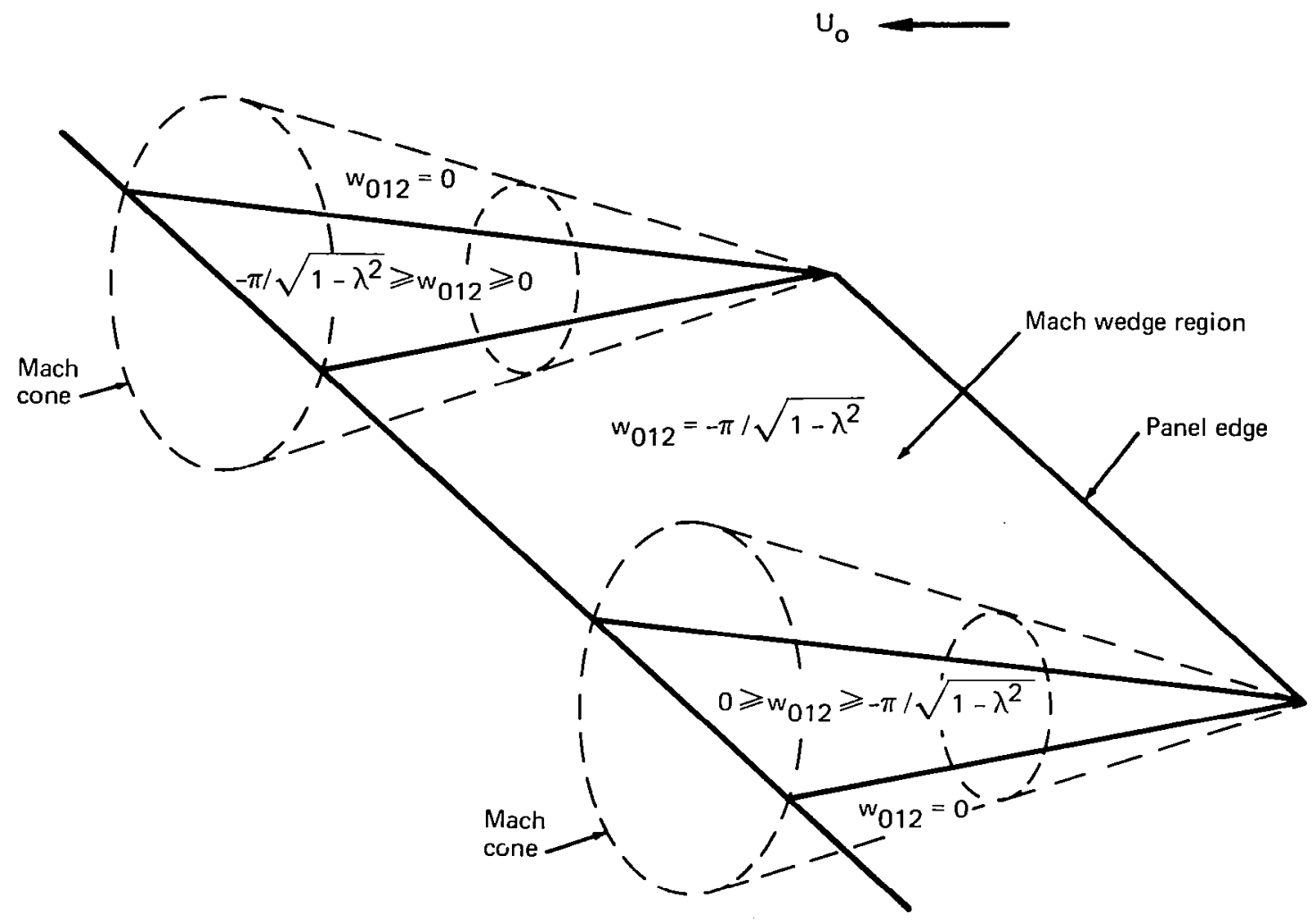

Figure 92. - Evaluation of wo For Both Endpoints of Panel Edge at Points on The Panel Plane 


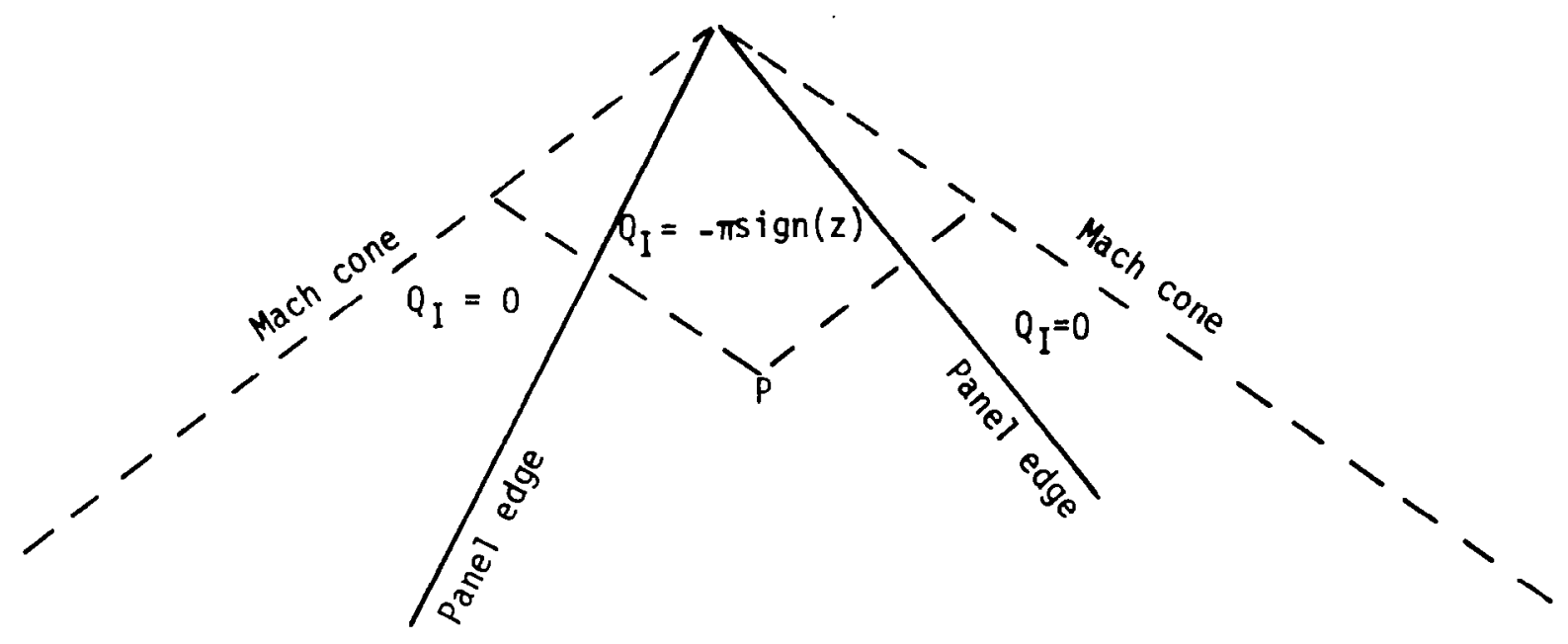

Figure 93.-Evaluation of $Q_{\text {I }}$ on The Panel For Panel Subsonic Leading Edges 


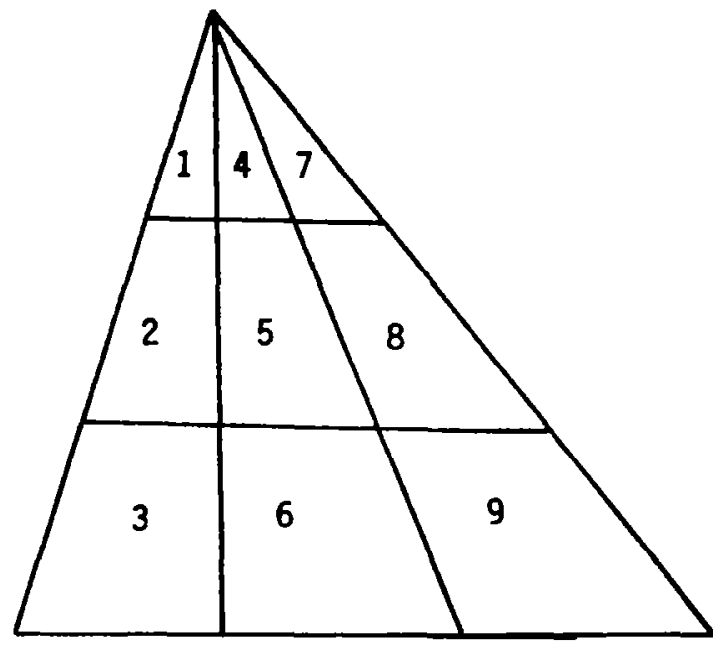

Panel No.

Other center panel values of source strength used in determining source strength in a given panel

1

2

5
$2,4,5$

$1,3,4,5,6$

$1,2,3,4,6,7,8,9$

Figure 94.-Panels Used in Determining Source Strength From Weighted Least Square Fit

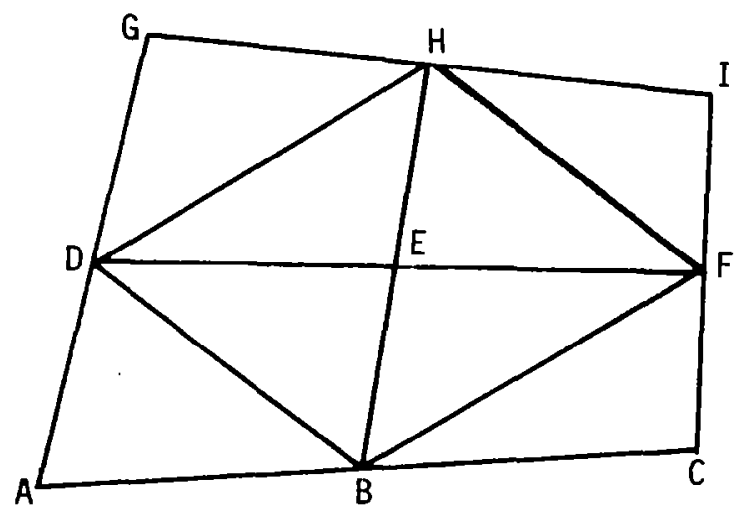

Figure 95.-IIlustration of Subpanels Into Which Basic Four Point Non-planar Panel is Divided 


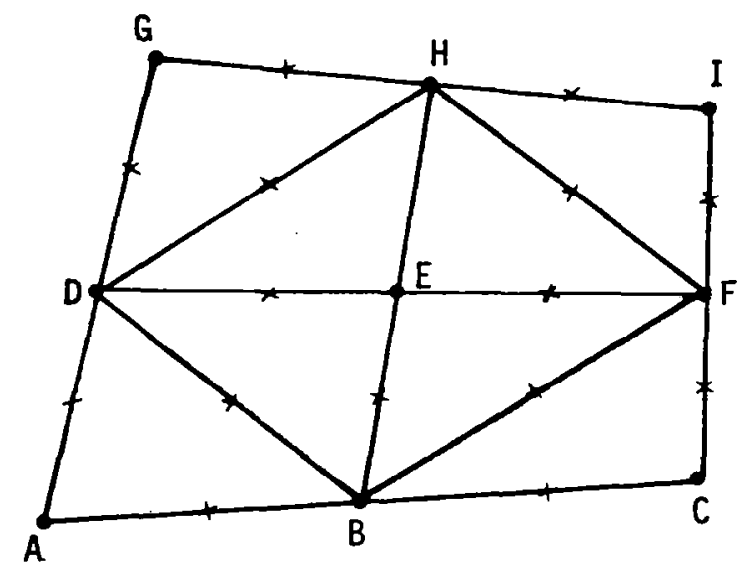

Figure 96.-IIlustration of Points at Which Doublet Strength Values Are Used to Define The Quadratic Distribution on Each Subpanel

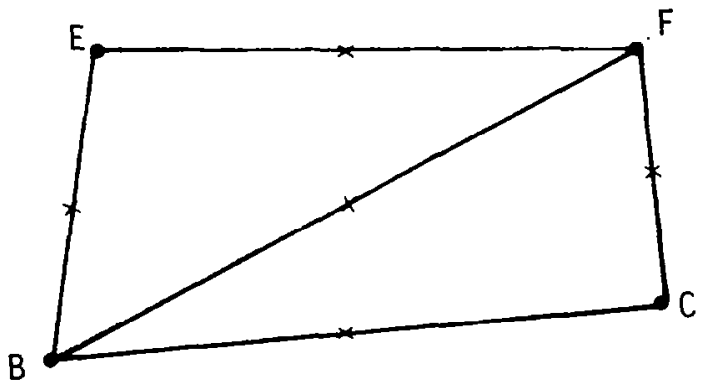

Figure 97. - IIlustration of How Continuity of Doublet Strength is Obtained at Subpanel Edges 


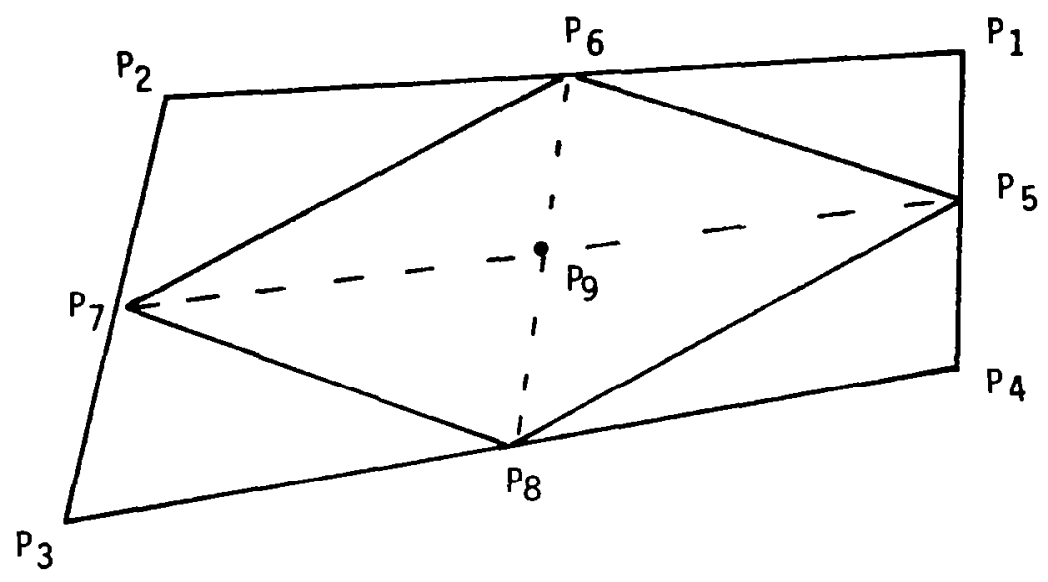

Figure 98. - Illustration of Panel Points Used to Measure Skewness of Panel 
a

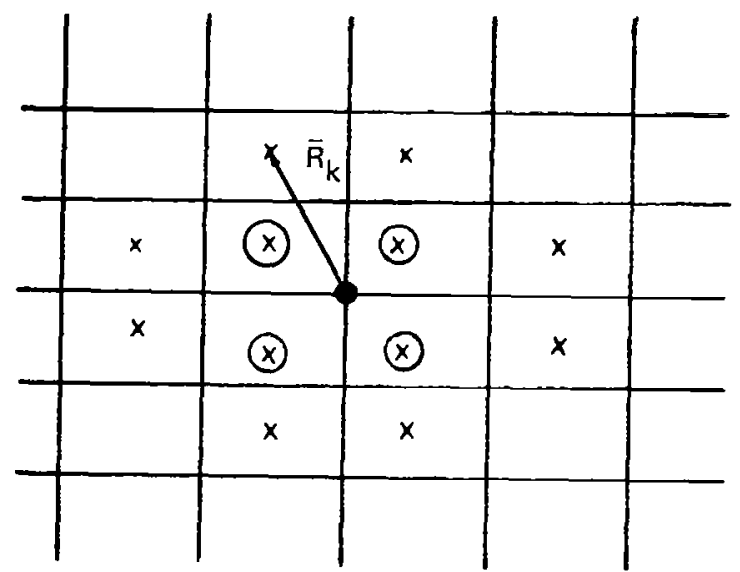

- Denotes point at which doublet strength is to be determined

(X) Denotes points at which doublet values are used with $w_{k}=1-M\left(1-\bar{R}_{k} \cdot \hat{C}\right)$

$X \quad$ Denotes points at which doublet values are used with $w_{k}=10^{3}$

b

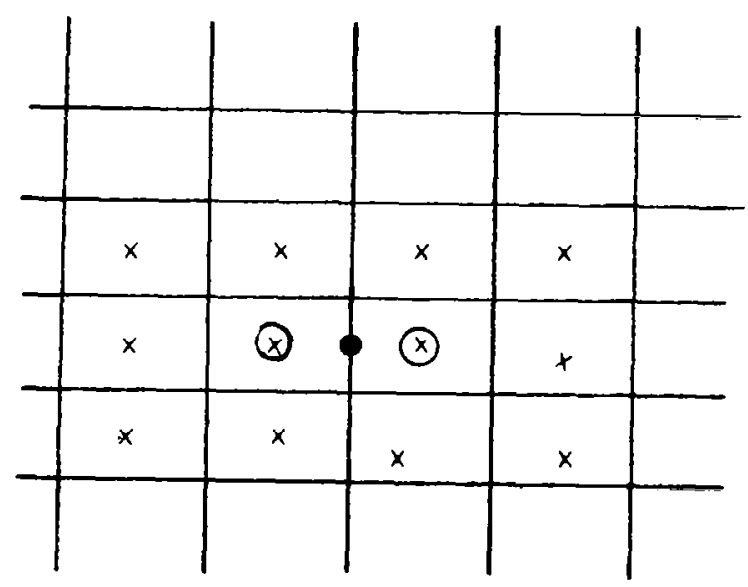

Figure 99. - Illustration of Determining Doublet Strength at Center Point of Panel Edge and at Panel Corner by Weighted Least Square Fit With Neighboring Center Panel Values For Interior Panels. 


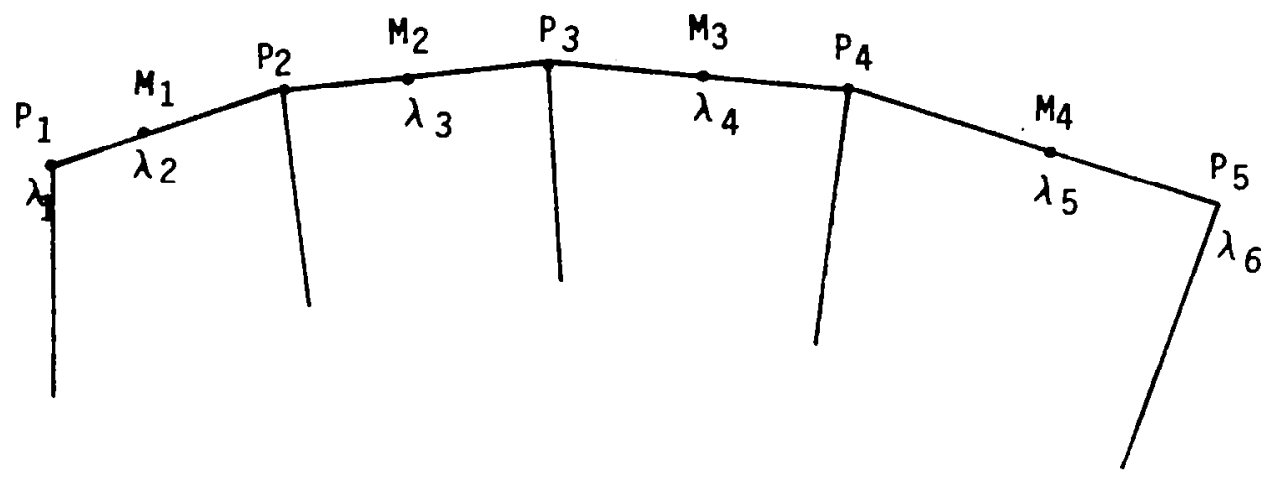

Figure 100.- I/lustration of Parameters and Points on a Network Edge Used in Interpolation 
- Denotes point at which doublet strength is to be determined

(X) Denotes points at which doublet values are used with weight $10^{3}$

$X$ Denotes points at which doublet values are used with $w_{k}=1-M\left(\bar{R}_{k} \cdot \hat{C}\right)$

a.)

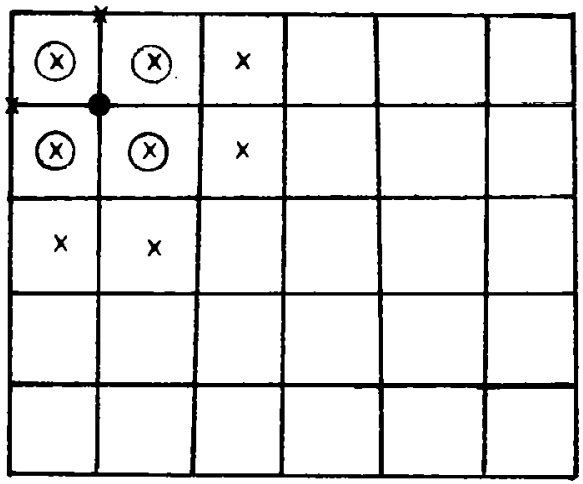

b.)

\begin{tabular}{|c|c|c|c|c|c|}
\hline$x$ & $\otimes$ & $\otimes$ & $x$ & & \\
\hline$x$ & $\otimes$ & $\otimes$ & $x$ & & \\
\hline & $x$ & $x$ & & & \\
\hline & & & & & \\
\hline & & & & & \\
\hline
\end{tabular}

d.)

\begin{tabular}{|l|l|l|l|l|l|}
\hline$\otimes$ & $\otimes$ & $x$ & & & \\
\hline$x$ & $x$ & $x$ & & & \\
\hline & & & & & \\
\hline & & & & & \\
\hline & & & & & \\
\hline
\end{tabular}

Figure 101.-IIlustration of Least Squares Fit Applied to Finding Corner And Midpoint Edge Values of The Doublet Strength For Panels Near Network Edges 


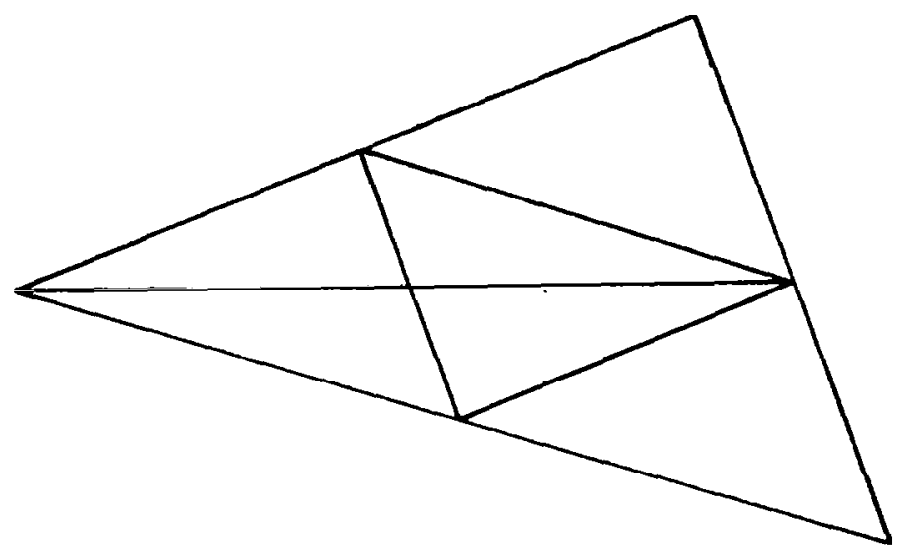

Figure 102.-Subpanels of Triangular Panel 


\section{REFERENCES}

(1) Ashley, H., and Rodden, W. P.: “Wing-Body Aerodynamic Interaction," Annual Review of Fluid Mechanics, Vol. 4, 1972, pp. 431-472.

(2) Rubbert, P. E., and Saaris, G. R.: "Review and Evaluation of Three Dimensional Lifting Potential Flow Analysis Method for Arbitrary Configurations," AIAA Paper 72-188, Jan., 1972.

(3) Johnson, F. T., and Rubbert, P. E.: "Advanced Panel-Typé Influence Coefficient Methods Applied to Subsonic Flow," AIAA Paper 75-50, Jan., 1975.

(4) Woodward, F. A.: "Analysis and Design of Wing-Body Combinations at Subsonic and Supersonic Speeds," Journal of Aircraft, Vol. 5, No. 6, Nov.-Dec. 1968, pp. $528-534$.

(5) Woodward, F. A.: "An Improved Method for the Aerodynamic Analysis of WingBody-Tail Configurations in Subsonic and Supersonic Flow, Part I - Theory and Application," NASA CR 2228, Part I (1973).

(6) Mercer, J. E., Weber, I. A., and Iesferd, E. P.: "Aerodynamic Influence Coefficient Method Using Singularity Splines," NASA CR 2423, 1974.

(7) Morino, L, Chen, L. T., Suciu, E. O.: "Steady and Oscillatory Subsonic and Supersonic Aerodynamics Around Complex Configurations," AIAA Journal, Vol. 13, pp. 368-375 (1975).

(8) Ehlers, F. E., and Rubbert, P. E.: "A Mach Line Panel Method for Computing the Linearized Supersonic Flow Over Planar Wings,” NASA CR 152126 (1978).

(9) Hess, John: "Calculation of Potential Flow About Arbitrary 3-D Lifting Bodies," Douglas Report MDCJ-5679-01.

(10) Ward, G. N.: “Linearized Theory of Steady High-Speed Flow," Cambridge University Press (1955).

(11) Goethert, B.: "Plane and Three-Dimensional Flow at High Subsonic Speeds," N.A.C.A. TM 1105.

(12) Hadamard, J: "Lectures on Cauchy's Problem in Linear Partial Differential Equations," Yale University Press (1928).

(13) Heaslet, M. A., and Lomax, H.: "The Use of Source-Sink and Doublet Distributions Extended to the Solution of Boundary-Value Problems in Supersonic Flow," NACA Report No. 900 (1948). 
(14) Heaslet, M. A., Lomax, H., and Jones, A. L.: "Volterra's Solution of the Wave Equation as Applied to Three-Dimensional Supersonic Airfoil Problems," NACA Report No. 889 (1947).

(15) Robinson, A.: "On Source and Vortex Distributions in the Linearized Theory of Steady Supersonic Flow," Quart, J. Mech. Appl. Math. I, 408-32. (1948)

(16) Courant, R.: "Differential and Integral Calculus," Vol. II, Nordeman Publishing Company, New York (1936).

(17) Kopal, Z.: "Tables of Supersonic Flow Around Cones," Tech. Report No. 1, M.I.T. (1947).

(18) Kopal, Z.: "Tables of Supersonic Flow Over Yawing Cones," Tech. Report No. 3, M.I.T. (1949).

(19) Kopal, Z.: "Tables of Supersonic Flow Around Cones of Large Yaw," Tech. Report No. 5, M.I.T. (1949).

(20) Roberts, R. C., and Riley, J. D.: "A Guide to the Use of the M.I.T. Cone Tables," Journal of the Aeronautical Sciences, Vol. 21, p. 336-342 (1954).

(21) Van Dyke, M. D.: "The Slender Elliptical Cone as a Model for Non-Linear Supersonic Flow Theory," J. Fluid Mech. Vol. 1, pt. 1, May, 1956, pp. 1-15.

(22) Maslen, S. H.: "Pressure Distribution on Thin Conical Body of Elliptic Cross Section at Mach Number 1.89," NACA RM E8K05, January, 1949.

(23) Jones, R. T. and Cohen, D.: "High Speed Wing Theory," Princeton University Press (1960).

(24) Shapiro, A. H.: "The Dynamics and Thermodynamics of Compressible Fluid Flow," Vol. I, The Ronald Press Company, New York (1953).

(25) Lighthill, M. J.: "Supersonic Flow Past Slender Bodies of Revolution, the Slope of Whose Meridian Section is Discontinuous," Quarterly Journal of Mechanics and Applied Mathematics, Vol. I, 90, (1948).

(26) Carlson, H. W.: "Aerodynamic Characteristics at Mach Number 2.05 of a Series of Highly Swept Arrow Wings Employing Various Degrees of Twist and Camber," NASA Tech. Mem. X-332 (1960).

(27) Carlson, H. W.: "Pressure Distributions at Mach Number 2.05 on a Series of Highly Swept Arrow Wings Employing Various Degrees of Twist and Camber," NASA Tech. Note D-1264 (1962).

(28) D'Attore, L., Bilyk, M. A., and Sergeant R. J.: “Three Dimensional Supersonic Flow Field Analysis of the B-1 Airplane by a Finite Difference Technique and Comparison with Experimental Data," AIAA Paper 74-189 (1974). 
(29) Gapcynski, J. P. and Landrum, E. J.: "Tabulated Data from a Pressure-Distribution Investigation at Mach Number 2.01 of a $45^{\circ}$ Sweptback-Wing Airplane Model at Combined Angles of Attack and Sideslip," NASA Memorandum 10-15-58L (1958).

(30) Manro, Marjorie E.: "Supersonic Pressure Measurements and Comparison of Theory to Experiment for an Arrow-Wing Configuration," NASA CR-145046 (1976).

(31) Spurlin, C. J.: "Documentation of Wind Tunnel Test Data From the AFFDL Advanced Supersonic Configuration Test," Arnold Engineering Development Center Report AEDC-DR-76-96 (Dec., 1976).

(32) Moran, J., Tinoco, E. N., and Johnson, F. T.: “User's Manual-Subsonic/Supersonic Advanced Panel Pilot Code," NASA CR-1 52047 (1978). 


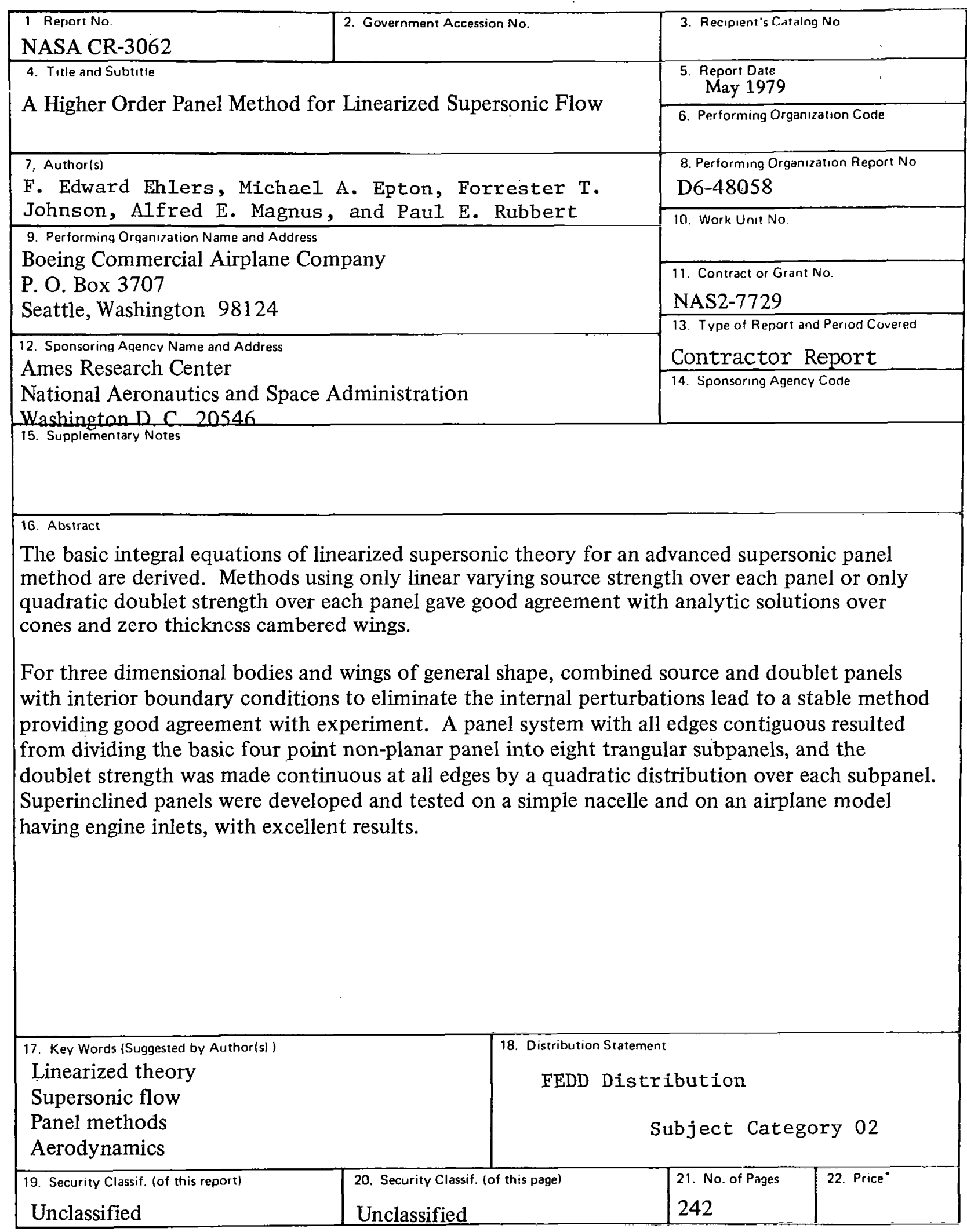

Available: NASA'ś Industrial Application Centers 